\title{
STRESS ET ADAPTATION CHEZ L'ENFANT
}

\author{
Sous la direction de
}

\section{Michelle Dumont et Bernard Plancherel}

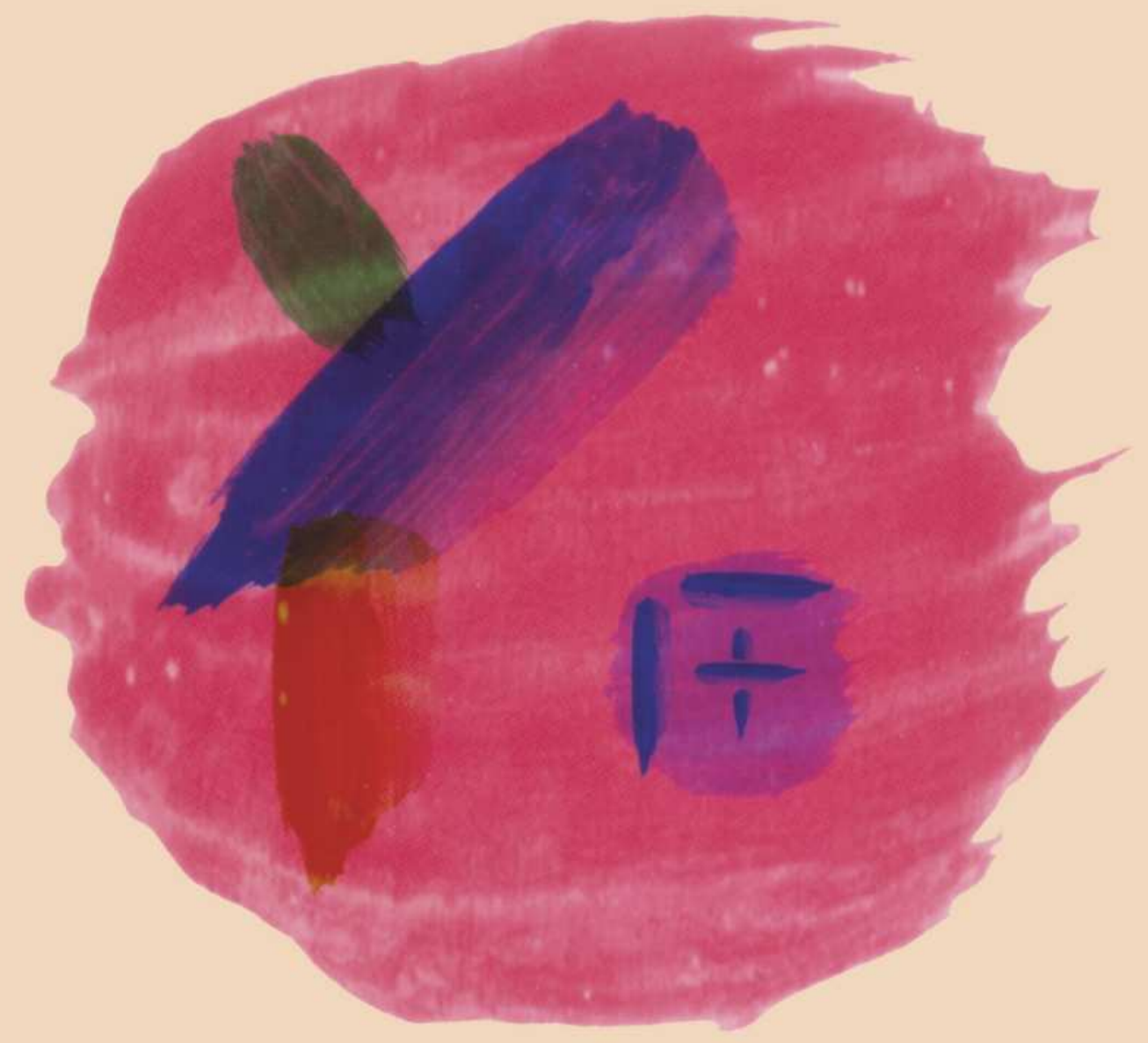

$\square$ Presses de I'Université du Québec 



\section{STRESS ET ADAPTATION CHEZ L'ENFANT}


Collection sous la direction de

Réjean Tessier et George M. Tarabulsy

Attachement et développement • Le rôle des premières relations dans le développement humain

Sous la direction de George M. Tarabulsy, Simon Larose, David R. Pederson et Greg Moran 2000, ISBN 2-7605-1091-3, 420 p.

Enfance et milieux de vie - Initiatives communautaires novatrices

Sous la direction de Jean-Pierre Garnier et Claire Chamberland

2000, ISBN 2-7605-1069-7, 120 p.

Le tempérament de l'enfant • Cing études

Sous la direction de George M. Tarabulsy, Réjean Tessier et Arvid Kappas 1998, ISBN 2-7605-1012-3, 164 p.

Enfance et famille - Contextes et développement

Sous la direction de George M. Tarabulsy et Réjean Tessier

1997, ISBN 2-7605-0912-5, $180 \mathrm{p}$.

\section{Dimensions de la maltraitance}

Sous la direction de Réjean Tessier, George M. Tarabulsy et Louise S. Éthier 1996, ISBN 2-7605-0911-7, $124 \mathrm{p}$.

Le modèle écologique dans l'étude du développement de l'enfant

Sous la direction de Réjean Tessier et George M. Tarabulsy

1996, ISBN 2-7605-0906-0, $130 \mathrm{p}$.

Le développement émotionnel et social de l'enfant

Sous la direction de George M. Tarabulsy et Réjean Tessier

1996, ISBN 2-7605-0897-8, 148 p.

\section{PRESSES DE L'UNIVERSITÉ DU QUÉBEC}

Le Delta I, 2875, boulevard Laurier, bureau 450

Sainte-Foy (Québec) G1V 2M2

Téléphone: (418) 657-4399 • Télécopieur: (418) 657-2096

Courriel: puq@puq.uquebec.ca - Internet : www.puq.uquebec.ca

Distribution :

\section{CANADA et autres pays}

DISTRIBUTION DE LIVRES UNIVERS S.E.N.C.

845, rue Marie-Victorin, Saint-Nicolas (Québec) G7A 3S8

Téléphone : (418) 831-7474/1-800-859-7474 • Télécopieur : (418) 831-4021

\section{FRANCE}

DIFFUSION DE L'ÉDITION QUÉBÉCOISE 30, rue Gay-Lussac, 75005 Paris, France

Téléphone: 33143544902

Télécopieur: 33143543915

\section{SUISSE}

GM DIFFUSION SA

Rue d'Etraz 2, CH-1027 Lonay, Suisse

Téléphone: 0218032626

Télécopieur : 0218032629 


\section{STRESS ET ADAPTATION CHEZ L'ENFANT}

Sous la direction de

Michelle Dumont et Bernard Plancherel 
Données de catalogage avant publication (Canada)

Vedette principale au titre :

Stress et adaptation chez l'enfant

(Collection D'Enfance; 8)

Comprend des réf. bibliogr. et un index.

ISBN 2-7605-1124-3

1. Stress chez l'enfant. 2. Adaptation chez l'enfant. 3. Gestion du stress chez l'enfant. 4. Élasticité chez l'enfant. 5. Stress. 6. Adaptation (Psychologie). I. Dumont, Michelle. II. Plancherel, Bernard. III. Collection.
BF723.S75S77 2001
$155.4^{\prime} 18$
C2001-940304-6

Nous reconnaissons l'aide financière du gouvernement du Canada par l'entremise du Programme d'aide au développement de l'industrie de l'édition (PADIÉ) pour nos activités d'édition.

Révision linguistique: Le GRAPHE ENR.

Mise en pages : CARACTÉRA PRODUCTION GRAPHIQUE

Couverture: Caron \& Gosselin Communication GraphiQue

123456789 PUQ 2001987654321

Tous droits de reproduction, de traduction et d'adaptation réservés (C) 2001 Presses de l’Université du Québec

Dépôt légal $-2^{\mathrm{e}}$ trimestre 2001

Bibliothèque nationale du Québec / Bibliothèque nationale du Canada Imprimé au Canada 


\section{TABLE DES MATIÈRES}

Remerciements

$\mathrm{xi}$

Introduction: Stress et adaptation chez l'enfant

dans ses environnements

Michelle Dumont et Bernard Plancherel......... 1

Chapitre 1: Le stress psychologique

Bernard Plancherel ..................... 9

Définition et histoire du concept de stress ....... 11

Le modèle transactionnel . . . . . . . . . . . . . . . . 13

Le stress sur le plan physiologique ............ 14

Stress et santé ...................... 16

Les sources de stress................... 17

Le stress des événements existentiels. . . . . . . . . . 19

Les maladies du stress . . . . . . . . . . . . . . 22

Données de recherche................. 23

Conclusion....................... 26

Chapitre 2: Régulation biocomportementale des réponses d'adaptation au stress chez les jeunes enfants Marcel Trudel, Élisabeth Chaussegros de Léry et Guadalupe Puentes-Neuman . . . . . . . . . . . . . 29

Perspective physiologique de $l^{\prime}$ adaptation au stress ................. 32

Les mécanismes physiologiques associés au stress . . 33

La régulation physiologique associée à l'adaptation comportementale $\ldots \ldots \ldots \ldots \ldots \quad 36$ 
La relation entre l'adaptation comportementale et la régulation physiologique chez les enfants en groupe de pairs ................... 40

L'adaptation dans un nouveau groupe de pairs................. 41

L'adaptation auprès des pairs en groupe stable.................. 44

L'adaptation biocomportementale chez les enfants atypiques ............ 46

Bilan critique et perspectives de recherche....... 48

Chapitre 3: Les stratégies adaptatives Michelle Dumont ..................... 53

Définitions et modèles théoriques. ............ 56

Styles de coping et degré d'efficacité .......... 59

Approche développementale et relation coping-santé mentale............. 64

Le coping cognitif .................. 64

Le coping social................. 65

Le coping émotionnel . . . . . . . . . . . . 67

Conclusion........................ 67

Chapitre 4: Les stratégies d'adaptation de la mère et de son enfant dans le phénomène de résilience Marc A. Provost, Michelle Dumont, Sylvain Coutu et Nicole Royer. . . . . . . . . . . . . . 69

Méthode............................. 75

Les participants................. 75

Première étape : le choix de la population ..... 75

Deuxième étape: le choix des participants.... 76

Les questionnaires pour la formation

des groupes.................... 76

La formation des quatre groupes ......... 78

Les instruments de mesure . . . . . . . . . . . . 79

Les procédures .................. 80

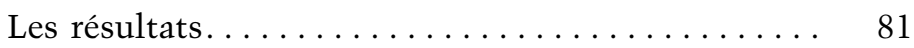

La réduction des données............. 81

Les résultats d'analyse .............. 82 
Discussion ........................ 84

Les stratégies d'adaptation de la mère ....... 86

Les stratégies d'adaptation des enfants....... 87

Les implications pour l'intervention........ 88

Chapitre 5: Stress familial, stratégies adaptatives et déficience intellectuelle

Jean-Luc Lambert et Romain Lanners . . . . . . . . . . . 91

L'évolution des perspectives................ 93

La détresse chronique ................ 94

Le stress et les ressources ............. 96

Une perspective écologique ............. 102

Les demandes des parents et le partenariat ... 107

Un modèle pour l'adaptation . . . . . . . . . . . . . . 112

Stress et ressources . . . . . . . . . . . . 113

Les relations avec l'écologie familiale....... 115

Des stratégies de coping............. 116

L'adaptation familiale: un système complexe . . 121

Une piste à privilégier:

le coping chez les enfants déficients............ 124

Chapitre 6: Dépistage et utilisation des stratégies d'adaptation chez les enfants et les adolescents souffrant de maladie chronique: implications pratiques pour le travail clinique

Anthony Spirito et Deidre L. Donaldson . . . . . . . . . 129

Le Kidcope............................ 132

Les données normatives du Kidcope:

les différences quant à l'âge, au sexe

et aux stresseurs ................... 134

Les résultats du Kidcope:

échantillons cliniques............... 136

L'adaptation des enfants face

à une maladie chronique . . . . . . . . . . . . . 138

Implications cliniques. . . . . . . . . . . . . . 142

Différences de l'adaptation selon la situation ..... . 142

Implications cliniques. . . . . . . . . . . . . . 145 
Stratégies simples d'adaptation

ou patterns de stratégies d'adaptation. . . . . . . . 145

Implications cliniques................. 145

Évaluation actuelle de l'adaptation

dans des populations pédiatriques........... 146

Implications cliniques. . . . . . . . . . . . . . 149

Conclusion....................... 151

Notices biographiques. . . . . . . . . . . . . . . . . . . 155

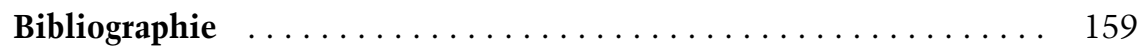

Index onomastique $\ldots \ldots \ldots \ldots \ldots \ldots \ldots \ldots \ldots \ldots \ldots \ldots \ldots \ldots \ldots$

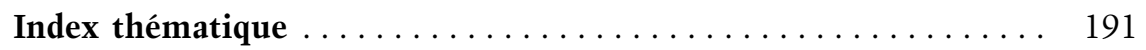




\section{REMERCIEMENTS}

La réalisation du présent ouvrage a requis la participation de plusieurs personnes. À cet égard, nous devons souligner le rôle indispensable de George M. Tarabulsy tant pour ses suggestions dans la réalisation de cette monographie que pour sa lecture attentive. Nous aimerions aussi remercier Isabelle Hémond pour le travail de traduction ainsi que Josée Bergeron pour sa participation à la création de l'index thématique. Enfin, il faut remercier nos éditeurs aux Presses de 1'Université du Québec, Mmes Angèle Tremblay et Marie-Noëlle Germain qui ont toujours soutenu la collection D'Enfance et qui ont su, par leur patience et leurs encouragements, mener ce travail à terme. 



\section{Stress et adaptation chez l'enfant dans ses environnements}

MiCHELLE DUMONT

Département de psychologie

Université du Québec à Trois-Rivières

Trois-Rivières (Québec)

BERNARD PLANCHEREL

Département de psychologie Université de Fribourg

Fribourg (Suisse) 



\begin{abstract}
De tout temps le stress a existé, qu'il soit causé par les intempéries, la famine, les guerres, les épidémies, la menace de prédateurs, etc. Nos existences sont toutefois plus complexes qu'autrefois car bien souvent, plutôt que d'apporter repos et sommeil, la tombée de la nuit devient l'occasion de s'adonner à diverses activités toutes aussi stimulantes les unes que les autres, ce qui réduit les périodes de récupération. Que l'on soit un adulte ou un enfant, un animal ou même une plante, tout organisme vivant est susceptible de vivre un état de stress lorsqu'il voit son homéostasie rompue. Ce livre a pour but de rapporter certains constats issus des travaux de recherche des dernières années dans le domaine du stress, individuel et familial, et de l'étude des stratégies adaptatives ( “coping ") adoptées en réponse aux situations stressantes. Cette monographie propose de définir les concepts de stress biologique ${ }^{2}$, de coping et de résilience, et de les illustrer par différents travaux de recherche réalisés auprès des enfants et de leur famille. Plus spécifiquement, les études présentées dans cet ouvrage décrivent certaines des stratégies adoptées par les enfants et leurs familles confrontés à des situations particulièrement stressantes, dont la maladie physique, la déficience intellectuelle d'un enfant, les facteurs de stress familial et l'intégration à un groupe de pairs.
\end{abstract}

Historiquement, c'est à partir des travaux de Cannon (1935) mais surtout de la contribution substantielle de Selye (1953) que le développement de ce domaine de recherche a pris son essor. D'abord traité d'un point de vue biologique, le domaine du stress fut par la suite étudié d'un point de vue psychologique, dans les années 1960, avec Lazarus puis, de façon plus marquée, à partir de la publication de l'ouvrage de référence de Lazarus et Folkman (1984). Pour Selye, chaque fois que notre environnement change, surtout lorsqu'il nous soumet à des pressions ou des

1. La notion de stratégie adaptative est une traduction du concept de “coping" utilisé en langue anglaise pour désigner la manière de faire face au stress, la manière de s'adapter aux situations problématiques.

2. Nous utiliserons de façon interchangeable les notions de stress biologique et de stress physiologique pour désigner la réponse physique d'un organisme à un stimulus stressant. 
exigences accrues, il déclenche en nous une réaction biologique de stress. En revanche, pour Lazarus, le stress se manifeste lorsque nous sommes confrontés à des événements qui, selon nous, mettent en danger notre bien-être et devant lesquels nous pensons ne pas avoir les ressources suffisantes pour réagir. L'analyse des ouvrages sur le sujet nous amène à distinguer trois façons de concevoir le stress: le stress comme stimulus environnemental (agent externe: désastres, événements de vie et tracas quotidiens); le stress comme réponse subjective (état interne de tension et évaluation subjective d'une situation jugée stressante); et le stress comme réponse biologique de l'organisme à un stimulus ou à une situation (réactions nerveuses et hormonales). Soulignons d'entrée de jeu qu'il est souvent difficile de tenir compte simultanément de ces trois points de vue théoriques, en particulier lorsqu'il s'agit d'enfants chez lesquels l'accès aux réponses physiologiques et à la signification accordée aux situations stressantes est beaucoup plus limité.

Devant un changement, l'individu ne reste habituellement pas passif par rapport à ce qui lui arrive: il essaie de faire face. On parle alors de " coping " ou de stratégie adaptative pour désigner la façon de s'ajuster aux situations difficiles. Ce terme implique l'existence d'un problème réel ou imaginé et la mise en place d'une réponse pour faire face à une situation stressante qui nécessite une adaptation (Paulhan et Bourgeois, 1995). Cette monographie met en évidence la diversification des réponses biologiques, comportementales, cognitives et psychologiques utilisées pour rétablir l'homéostasie d'un organisme subissant un déséquilibre par suite d'un stress endogène ou exogène. Comme dirait Selye, si nous ne devons ni ne pouvons éviter le stress, nous pouvons cependant apprendre à nous en accommoder et même à le trouver agréable, à la condition toutefois d'ajuster notre philosophie de la vie.

La réponse aux différentes sources de stress a pour objectif de nous stimuler à une meilleure adaptation. Le relevé des études dans le domaine du stress psychologique nous amène à constater des différences individuelles importantes dans la signification accordée aux situations stressantes et dans la manière $d^{\prime} y$ faire face. Autrement dit, le même environnement peut être jugé stressant pour un individu qui n'a pas les moyens de s'y adapter, mais confortable ou stimulant pour un autre qui possède les capacités nécessaires (Aldwin, 1994). Ces différentes réponses dépendent des conditions de vulnérabilité de la personne vis-à-vis des facteurs de stress, conditions qu'on mesure généralement en fonction de prédispositions psychologiques, biologiques et environnementales pouvant être ou pas génératrices de situations stressantes. Or, selon plusieurs résultats de recherche, la résistance d'une personne au stress se développe 
suivant la présence plus ou moins élevée de facteurs de protection (soutien social et familial satisfaisant, estime de soi positive, disposition à l'optimisme). Lorsqu'une personne bénéficiant de ces facteurs de protection fait face à plusieurs facteurs d'adversité sans présenter de signes de détresse, on parle d'un phénomène de "résilience ". Bien que l'étude de la résilience en psychologie tire son origine de l'intérêt porté aux mécanismes adaptatifs que durent déployer les survivants de l'holocauste lors de la Deuxième Guerre mondiale, elle ne fait l'objet d'un intérêt scientifique marqué que depuis une vingtaine d'années.

Centré sur l'étude du stress et des stratégies adaptatives durant l'enfance, le présent ouvrage se divise en deux parties. La première introduit les notions théoriques de stratégie adaptative et de stress, biologique et psychologique. La seconde présente des chapitres à caractère empirique traitant de l'adaptation des enfants face au stress familial et à la maladie.

Bernard Plancherel donne, dans le premier chapitre, une description générale de la notion de stress psychologique. Un bref rappel historique permet de voir comment on est arrivé à la notion de stress communément admise aujourd'hui, qui fait du stress un ensemble de transactions entre la personne et son environnement, et met l'accent autant sur les cognitions et émotions que sur l'action. Ce modèle "transactionnel ", développé par Lazarus, sert d'arrière-plan conceptuel à toutes les recherches actuelles sur le stress. Le chapitre résume ensuite les principaux thèmes qui ont suscité l'intérêt des chercheurs dans le domaine du stress : le stress et la santé, les sources de stress, la recherche sur les événements existentiels $^{3}$, les tracas quotidiens et les tensions chroniques, les différentes maladies du stress. D'après les données de recherche, dont certaines sont déjà anciennes, il ne fait de doute pour personne que le stress peut causer des troubles divers, physiques comme psychiques, et cela à tout âge de la vie. Il n'en demeure pas moins vrai que les études sur le stress, comme le rappelle la conclusion, se sont trouvées confrontées à divers problèmes méthodologiques. On a dû prendre en compte différentes variables modératrices (facteurs de protection ou de vulnérabilité, résilience), si bien que l'approche du stress psychologique est aujourd'hui presque exclusivement celle du "faire face " (ou coping), comme on pourra le voir dans les chapitres suivants.

3. Nous utiliserons de façon interchangeable le concept d'événement de vie /traduction du concept de “ life events »), communément utilisé en Amérique du Nord, et la notion d'événement existentiel, plus populaire en Europe. 
Marcel Trudel, Élisabeth Chaussegros de Léry et Guadalupe PuentesNeuman traitent, dans le deuxième chapitre, le concept de stress physiologique. À l'origine, les travaux de Cannon, mais en particulier ceux de Selye, ont permis de présenter le stress biologique comme une réponse non spécifique à une demande psychologique ou physique faite à un organisme. Les réactions biologiques de stress sont destinées à préparer à l'action physique rapide ou encore à résister à la source de stress. Il y aurait donc un lien entre stress biologique et coping. Il s'agit ici d'un chapitre à la fois théorique et empirique. Dans la première partie de ce chapitre, les auteurs présentent le cadre théorique des mécanismes physiologiques associés à l'adaptation au stress. Pour ce faire, ils décrivent les deux systèmes neuroendocriniens qui sont impliqués dans une situation de stress (première voie: activation de la glande médullosurrénale qui sécrète les catécholamines; deuxième voie: activation de l'axe hypothalamiquepituitaire-surrénal qui sécrète les glucocorticoïdes) et qui influencent les réponses émotionnelles et cognitives de l'individu. Dans la seconde partie de ce chapitre, de nature plus empirique, il sera question de travaux de recherche ayant mis en relation l'adaptation comportementale (styles sociaux d'interaction) et la régulation physiologique (mesures endocriniennes) chez des enfants en groupe de pairs. Cette situation de groupe représente une source de stress social puisque les enfants ne se connaissent pas. Une analyse critique au plan méthodologique permet de mieux comprendre les résultats obtenus dans ce type d'études faisant appel à des mesures physiologiques auprès de très jeunes enfants.

Michelle Dumont traite, dans le troisième chapitre, la notion de stratégie adaptative en rapport avec la théorie du stress psychologique suggérée par Lazarus et Folkman (1984). Il sera question de définir le concept de “ coping " à l'intérieur de deux modèles théoriques dominants: l'approche contextuelle et l'approche psychodynamique. Notons ici que l'approche contextuelle domine le point de vue théorique actuel, ce qui influence l'élaboration des instruments de mesure permettant d'évaluer le coping d'un point de vue ontogénétique. Un autre constat se dégage du relevé des ouvrages sur le sujet, à savoir que les facteurs de stress peuvent affaiblir les capacités d'adaptation des individus au point d'engendrer des difficultés psychologiques. En effet, lorsque la menace est grande et que l'habilité à y faire face est faible, une personne ressentira plus de stress; alors que lorsque l'efficacité du coping est grande, le stress peut être ressenti beaucoup moins fortement (Taylor, 1995). C'est dans ce contexte que l'efficacité du coping et ses conséquences sur la santé mentale / la détresse psychologique seront introduites. Dans ce chapitre théorique, il sera donc question du lien stress-détresse et des changements observés dans la réponse de coping au cours de l'enfance. 
Marc Provost, Michelle Dumont, Sylvain Coutu et Nicole Royer évaluent, dans le quatrième chapitre, la notion de résilience en lien avec les stratégies d'adaptation de la mère et de son enfant. Dans le domaine de la physique, la notion de résilience fait référence à l'aptitude d'un corps à résister à un choc. Cela correspond à une première définition proposée en psychologie pour qualifier les individus résilients d'invulnérables, d'invincibles. Cette conception de la résilience est peu à peu abandonnée puisqu'elle n'est pas une caractéristique définitive car selon le contexte et le temps, la résistance au stress peut être érodée par les circonstances adverses qui se sont accumulées ou les échecs subis au cours du développement. La résilience d'un enfant reflète la présence de facteurs protecteurs qui forment un tampon contre les effets néfastes des agents stresseurs. En ce sens, Masten et Garmezy (1985) croient qu'en présence de caractéristiques positives de la personnalité (estime de soi positive, optimisme, tempérament facile, etc.), de cohésion et de chaleur familiales ou encore de soutien social extérieur, les problèmes que rencontrent les enfants exposés à l'adversité psychosociale vont se résoudre d'une manière favorable. Ce chapitre empirique étudie le concept de résilience en relation avec les stratégies adaptatives de la mère et de son enfant en fonction du stress familial. Cette recherche constitue une étape de plus dans l'identification des ressources personnelles, familiales et environnementales susceptibles d'augmenter la résilience d'un individu face à l'adversité.

Jean-Luc Lambert et Romain Lanners brossent, dans le cinquième chapitre, un tableau de l'évolution des connaissances sur le stress familial et l'adaptation sociale dans les familles avec enfant déficient intellectuel. À partir d'une analyse critique, les auteurs proposent d'expliquer le stress vécu par ces familles et les stratégies de coping qu'elles adoptent en soulignant l'importance des significations apportées à cette situation de stress. Ils présentent les modèles explicatifs de stress familial développés dans les années d'après-guerre ( “ $\mathrm{ABCX}$ » et “ $\mathrm{ACBX}$ »), ainsi que les éléments centraux du modèle écologique de Bronfenbrenner (1979) appliqués à ce type de famille. Les auteurs décrivent également un nouveau courant de recherche mettant en évidence les besoins de ces familles, et en particulier leurs demandes (d'informations, de soutien formel et informel, d'aide financière, d'intégration sociale de l'enfant). Leur analyse des modèles théoriques rendant compte du stress et du coping de ces familles souligne l'importance respective des variables suivantes: les besoins, les réactions émotionnelles, les stratégies instrumentales et cognitives, les ressources et les attributions de signification. Au fur et à mesure de leurs recherches, les auteurs ont développé une nouvelle approche englobant l'ensemble de ces variables. Ils ont eu recours à un instrument validé permettant l'observation du milieu 
familial avec enfant déficient intellectuel et à un questionnaire couvrant plusieurs stratégies cognitives et instrumentales. Finalement, les auteurs nous offrent plusieurs illustrations d'études de cas pour aborder le stress et le coping dans les familles avec enfant déficient au plan intellectuel.

Anthony Spirito et Deidre L. Donaldson proposent, dans le sixième chapitre, d'étudier, dans le travail clinique, les implications pratiques de l'utilisation d'un bref instrument de dépistage permettant d'évaluer les stratégies adaptatives cognitives et comportementales des enfants et des adolescents. Les auteurs présentent les différentes étapes qui ont permis l'élaboration d'une telle mesure, le Kidcope, ainsi que les résultats des études de fiabilité et de validité réalisées auprès de populations normales, afin d'en tester les propriétés psychométriques et d'établir les données normatives auxquelles l'adaptation des enfants souffrant de maladie chronique pourrait être comparée.

En résumé, le présent ouvrage intègre les efforts conceptuels et expérimentaux pour : 1) comparer les résultats de recherches dans les domaines $\mathrm{du}$ stress biologique et psychologique, du coping et de la résilience; 2) définir certaines cibles d'intervention pouvant améliorer le bien-être des enfants, normaux ou atypiques; et 3) étudier l'efficacité du coping face au stress familial, en groupe de pairs ou en présence d'une maladie. 


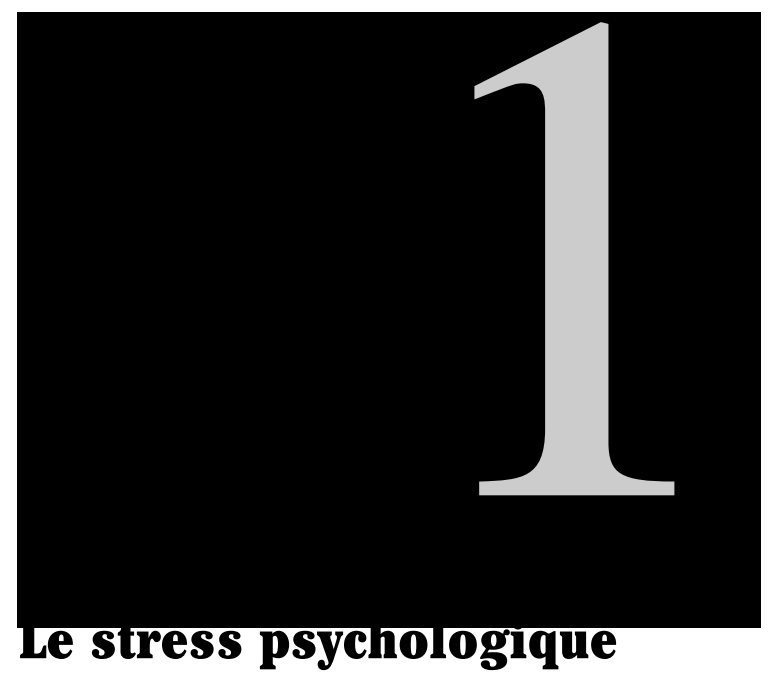

BERNARD PLANCHEREL

Département de psychologie

Université de Fribourg

Fribourg (Suisse) 

$\mathrm{R}$

Rendu responsable de plusieurs maladies physiques et psychiques, sujet d'innombrables articles et ouvrages scientifiques, le stress est devenu, comme on pouvait le lire dans un rapport du Bureau international du travail en 1993, “l'un des plus graves problèmes de notre temps, non seulement pour les individus dont il met en péril la santé physique et mentale, mais aussi pour les entreprises et les gouvernements ". Même si on ne le prend pas pour le nouveau malaise de la civilisation, on s'y intéresse comme à une nouvelle clé pour comprendre certains problèmes dans le domaine de la santé physique et psychique dans tous les âges de la vie. Le chapitre qui suit présente la notion de stress telle qu'elle est communément admise et donne un aperçu de la recherche en psychologie, et plus précisément de celle qui s'est penchée sur le stress durant l'enfance.

\section{DÉFINITION ET HISTOIRE DU CONCEPT DE STRESS}

Il y a plusieurs façons de définir le stress. L'une, qu'on appelle "stimulus based ", voit dans le stress une caractéristique aversive de l'environnement. Cette conception considère que certaines situations sont stressantes indépendamment des différences individuelles. L'approche dite “ response based " définit le stress comme une réponse physiologique de l'individu aux stimuli aversifs. Il y a enfin une conception du stress qui le définit comme processus. Dans cette conception, on prend en compte la façon dont les individus perçoivent les stresseurs, la réaction qui s'ensuit et comment ils tentent de faire face à ces situations. Cette approche est à la fois psychologique et psychophysiologique. Ces différentes conceptions du stress sont liées à l'histoire de sa découverte.

C'est en 1929 que Cannon fit l'hypothèse qu'une menace avait pour résultat une réaction de fuite ou de combat, et que cette réaction était une réaction au stress, qu'il soit physique ou social. Pour lui, le siège des émotions est dans le SNA (système nerveux autonome) et non dans l'activité viscérale comme le croyait James (Cannon, 1929). Il s'agit en 
fait du système sympathique, considéré comme responsable de la mobilisation des ressources de l'organisme pour se préparer à l'action, en réaction à un danger perçu. Les idées de Cannon furent généralisées par Selye (1956), en qui on voit le fondateur de la théorie du stress. Déjà en 1935, il découvre un syndrome général d'adaptation (SGA), qui serait la réponse de l'organisme pour toute agression subie (Selye, 1936). Selye n'utilisa le mot stress que dans les années 1950 pour signifier une réponse physiologique non spécifique à n'importe quelle agression. Sa définition du stress est: "Réponse non spécifique du corps à une demande "(Selye, 1956). La théorie de Selye est d'abord physiologique. Il conclut que les réactions biologiques qu'il observe représentent la réponse du corps aux stresseurs et sont des indicateurs objectifs du stress, qu'il définit comme une réponse générale du corps aux demandes qui lui sont faites.

Selon Selye, est appelé stress l'ensemble des réactions d'adaptation qui peuvent avoir des conséquences aussi bien positives (se maintenir en vie) que négatives, si elles sont trop intenses ou prolongées. Selye distinguait donc l' ' ceustress ", la bonne réponse qui permettait de survivre, et le "distress", la mauvaise réaction de l'organisme. Mais, comme on ne distingue plus le bon et le mauvais cholestérol, actuellement, dans le langage courant, stress est synonyme de mauvais stress, à savoir énervement, déprime, angoisse ou surmenage.

Un nouveau pas dans la recherche sur le stress sera accompli lorsqu'on abandonnera les modèles unidimensionnels, basés soit sur le stimulus, soit sur la réponse, pour un modèle interactif qui prend en compte la relation dynamique de l'individu avec son environnement et met l'accent autant sur les cognitions et émotions que sur l'action. On ne peut donc pas parler du stress isolément, mais l'on doit considérer comment l'individu perçoit le stress et la façon dont il gère toute situation stressante. Cette perspective avait déjà inspiré un courant venant de la psychologie du Moi, qui introduisit le concept de copingl dans la foulée des mécanismes de défense de la psychanalyse (Hann, 1977; Vaillant, 1971). Pour Vaillant le coping est compris comme un comportement d'adaptation flexible et efficace qui s'oppose aux comportements mal adaptatifs ou pathologiques. Il s'agit des mêmes mécanismes de défense, mais qui sont jugés mal adaptatifs lorsqu'ils évitent le conflit au lieu de le résoudre et aboutissent à une régression non nécessaire. C'est cependant

1. La notion de " coping " a été traduite en français par celle de " stratégies adaptatives " pour décrire la manière de faire face au stress, la façon de s'adapter aux situations problématiques. 
de Lazarus que se réclament la plupart des chercheurs qui s'intéressent au stress et au coping. Reprochant à Vaillant et Haan de s'intéresser à la gestion du stress, non pas comme à un processus mais comme à une structure ou un trait de personnalité, Lazarus a introduit le modèle dit transactionnel (Lazarus, 1966). On parle alors d'une influence bidirectionnelle entre la situation et la personne. La personne peut aussi influencer la situation. Il est clair que dans une telle conception, il ne faut pas tenir compte seulement des effets à court terme, mais aussi des processus à long terme.

Les recherches avec des animaux vont étendre les stresseurs à tous les événements qui suscitent un état d'attention, devant quelque chose de nouveau, d'incertain ou de déplaisant. Puis, la recherche avec les humains adultes incitera les chercheurs à diriger leurs travaux sur les événements existentiels ${ }^{2}$. On s'est ensuite intéressé à la problématique du stress chez les adolescents et les enfants, en utilisant les recherches sur les adultes comme modèle théorique pour expliquer le fonctionnement des enfants, faute de données empiriques.

\section{LE MODÈLE TRANSACTIONNEL}

Pour Lazarus (1966): “ Le stress apparaît lorsque la personne est confrontée à des demandes qui excèdent ses ressources d'ajustement. » On trouve aussi une définition proche chez Spielberger (1979): “ Le stress peut être défini comme l'ensemble des transactions entre la personne et son environnement dans lesquelles les stresseurs sont reliés aux réactions d'anxiété par la perception d'une menace. »

Dans le stress, il faut donc distinguer les stimuli, ou stresseurs engendrant le stress, les réponses de stress et, entre stresseurs et réponses, une série d'interactions: menace, impact, période post-impact. Pour le stress psychologique, il faut faire intervenir l'évaluation ou appraisal.

Dans les réponses au stress, on distinguera aussi:

1) les réponses physiologiques (arousal) comme la tension artérielle plus élevée, le rythme cardiaque plus rapide, la tension excessive des muscles, la respiration saccadée. À long terme, l'effet cumulatif de ces

2. La notion $\mathrm{d}^{\prime}$ “ événement existentiel » est une traduction française du concept life events, lequel englobe les événements de vie ou les stresseurs majeurs qui obligent l'individu à s'adapter. Nous utilisons de façon interchangeable les expressions "événements de vie " et "événements existentiels". 
réactions peut provoquer un ulcère d'estomac, par exemple, ou encore des troubles du sommeil et de l'appétit;

2) les réactions émotionnelles, principalement l'anxiété lorsque le stress est un stimulus aversif, ou la tendance à la dépression, lorsque le stress est une perte;

3) les réactions au plan cognitif: rumination, souci (contenu) et augmentation des capacités lorsque le stress apparaît, puis déclin lorsque le stress augmente.

Lazarus divise la perception du stress en deux moments : la première évaluation où le sujet évalue le danger du stresseur et la deuxième, où il évalue ses ressources et sa capacité à surmonter la situation stressante.

La première évaluation permet d'identifier le type de stresseur: il peut être neutre et donc sans impact sur le sujet, une menace (la réponse est la peur), une perte qui a déjà eu lieu et qui a entraîné des conséquences (souvent la dépression), un défi qui met le sujet devant le choix entre la maîtrise et la passivité.

Les facteurs suivants sont importants pour l'évaluation du stress: 1) la sévérité du stresseur, qui est la somme de l'importance du but à atteindre et de l'intensité de la carence; 2) le nombre de buts à atteindre; 3) l'imminence de l'événement; 4) le temps passé à se soucier d'un stresseur potentiel ; 5) la probabilité de l'événement: on a fait l'hypothèse que plus l'événement était probable, plus le stresseur serait évalué important; et 6) l'ambiguité qui rend la situation plus aversive, car le sujet ne peut se préparer.

\section{LE STRESS SUR LE PLAN PHYSIOLOGIQUE}

Petite glande à la base du cerveau, l'hypothalamus joue un rôle important dans la réponse du stress, surtout en tant qu'aiguilleur. Après avoir perçu et évalué le stimulus, l'hypothalamus ordonne la mise en branle de deux systèmes de réaction de notre organisme: le système nerveux sympathique et le système hypophysaire. Le premier système entre en action dans les situations d'urgence, exigeant une réaction d'adaptation rapide. Le système nerveux sympathique accélère la production par les glandes médullosurrénales des hormones comme l'adrénaline et la noradrénaline. L'adrénaline est appelée hormone de l'urgence. Elle a pour effets l'accélération 
du rythme cardiaque, pour amener le sang en plus grande quantité dans les organes, la libération du sucre stocké dans le foie, pour donner de l'énergie, et l'augmentation de la réaction d'éveil.

Le système hypophysaire entre en jeu lorsqu'il est nécessaire d'inhiber l'action ou dans des situations où il y a perte de contrôle. L'hypophyse fabrique de nombreuses hormones: stimulines sexuelles, endorphines qui calment la douleur. Mais dans le cas du stress, c'est l'hormone appelée ACTH qui est importante. Cette hormone stimule les glandes corticosurrénales, qui à leur tour sécrètent des hormones dont la principale est le cortisol, qui se caractérise avant tout par son action anti-inflammatoire. Il a aussi comme effet la reconstitution du stock du sucre libéré lors du stress d'urgence. Dans le cas du stress chronique, il y a augmentation de la production de cortisol, ce qui entraîne une diminution des défenses immunitaires, une plus grande sensibilité aux maladies allergiques et des troubles cutanés. On suppose aussi que cela pourrait être la cause de poussées d'ulcères, de certains rhumatismes, de certains diabètes, de l'obésité, et surtout de la dépression.

On peut synthétiser l'aspect physiologique du stress dans le tableau suivant (d'après Henry et Stephens, 1977, et Rivolier, 1989):

\begin{tabular}{lll}
\hline & Contrôle menacé & Perte de contrôle \\
\hline Comportement & $\begin{array}{l}\text { Actif } \\
\text { défense - fuite ou combat }\end{array}$ & $\begin{array}{l}\text { Passif } \\
\text { résignation - repliement }\end{array}$ \\
Action & $\begin{array}{l}\text { Système sympathique: } \\
\text { glandes médullo-surrénales }\end{array}$ & $\begin{array}{l}\text { Hypophyse : glandes } \\
\text { corticosurrénales }\end{array}$ \\
Houro-endocrine & Noradrénaline & ACTH \\
& $\begin{array}{l}\text { Adrénaline } \\
\text { Corticostérone } \\
\text { Testostérone }\end{array}$ & Corticostérone \\
& Catécholamines \\
& & Testostérone \\
\hline
\end{tabular}

Il est devenu courant d'utiliser la mesure du cortisol salivaire dans les recherches sur le stress (Ben-Aryeh, Roll, Kahana, Malberger, Szargel et Gutman, 1985; Hubert et de Jong-Meyer, 1989), bien que cela donne parfois des résultats statistiquement peu clairs, étant donné que, comme dans la plupart des mesures physiologiques, les résultats varient beaucoup 
d'un sujet à l'autre (Berger, Bossert, Krieg, Dirlich, Ettmeier, Schreiber et von Zerssen, 1987). Dans le chapitre suivant, Trudel, Chaussegros de Léry et Puentes-Neuman reviendront sur ce sujet.

\section{STRESS ET SANTÉ}

Plusieurs modèles ont tenté de rendre compte de l'effet du stress sur la santé.

Dans les modèles endocriniens, on fait l'hypothèse que la détérioration de la santé peut se faire par: a) un phénomène d'épuisement; b) une erreur de la réponse hormonale, réponse inutile et coûteuse, action immunosuppressive des corticoïdes; ou c) un déraillement sécrétoire des glucocorticoïdes au profit des hormones inflammatoires (Selye, 1956).

Dans le modèle immunologique, on pense que le stress a comme conséquence une diminution des défenses immunitaires, laquelle, combinée avec un vecteur spécifique (virus), causera la maladie. Cependant il est très difficile de trouver dans les recherches un effet linéaire aussi simple (Villemain, 1989). On a aussi avancé la théorie de l'activation soutenue et prolongée (Ursin, 1980): quand l'activation tonique se prolonge et ne décroît pas, il y a risque de pathologie.

Laborit proposait le modèle de l'inhibition de l'action (Laborit, 1979). Il existerait ainsi un système activateur du comportement, qui irait dans le sens de la lutte ou de la fuite avec agressivité, et un système inhibiteur du comportement, qui irait dans le sens de l'évitement passif. Le système nerveux central est capable de mémoriser les actions gratifiantes ou non. Mais l'espace contenant les objets gratifiants peut être occupé par d'autres individus cherchant à se gratifier avec les mêmes objets. D'où lutte avec échec. Si l'action n'aboutit pas à une gratification, il y a inhibition de l'action et angoisse.

On connaît aussi les modèles psychosociaux comme ceux de Rahe (1968) ou de Dohrenwend (1986) dans lesquels on fait intervenir, entre le stresseur, souvent un événement existentiel, et sa répercussion sur la santé, différents éléments jouant le rôle de filtres et pouvant soit augmenter l'effet du stress, soit le diminuer: perception du stresseur, défenses psychologiques, réseau social, caractéristiques personnelles. Des modèles de ce type ont donné naissance à toute une série de recherches sur les facteurs protecteurs comme le réseau social, l'estime de soi, la gestion du stress ou coping, et ont utilisé pour ce faire le modèle de l'effet tampon (ou effet buffer). Il est difficile de dire qui a parlé pour la première fois de l'effet tampon, mais il apparaît de plus en plus à partir des années 1970. 
On parle d'effet tampon (buffer) lorsque des ressources personnelles et environnementales permettent de réduire l'impact du stress sur le bienêtre des individus. L'effet du stress sur la santé est supposé être d'autant plus diminué que les ressources sont nombreuses. Les ressources sont soit internes, comme des prédispositions, des caractéristiques physiologiques ou psychologiques, soit externes, comme le milieu familial, le réseau et le support social. On parlera alors de variables modératrices considérées comme des ressources amortissant le stress. La situation classique est celle qui considère les ressources comme une variable dont l'action porte sur l'impact des stresseurs sur la détresse. On pourrait aussi considérer les stresseurs comme une variable dont l'action porte sur l'effet d'atténuation qu'ont les ressources sur la détresse. La procédure statistique habituelle pour tester ce type de modèle est la régression multiple ou l'analyse de variance avec un terme d'interaction. Mais si le modèle de modération est le plus souvent utilisé dans les recherches sur l'effet tampon, il n'est pas le seul. D'autres modèles sont construits avec des variables médiatrices (on parlera alors de modèle de médiation): les ressources agissent sur le stress (en en diminuant l'importance), lequel augmente la détresse, ou bien les stresseurs diminuent les ressources, qui elles atténuent la détresse. Mais on pourrait aussi faire l'hypothèse que les stresseurs mobilisent les ressources. Pour tester ce type de modèle, on utilise la régression à deux niveaux, une fois en prédisant la troisième variable à partir de la première, et une deuxième fois en y intégrant la variable intermédiaire. Si l'effet de la première sur la troisième diminue de façon importante, voire même totalement, lorsqu'on fait intervenir la variable intermédiaire, on pourra alors parler d'un effet de médiation de la variable intermédiaire. Aujourd'hui, avec l'aide de logiciels comme Lisrel ou Amos, on peut aussi tester les modèles de médiation, car ils sont un cas particulier relativement simple des systèmes d'équations structurales (Plancherel, Bolognini, et Nuñez, 1994).

\section{LES SOURCES DE STRESS}

Parmi les principales sources de stress, on mentionne celles qui ont un rapport avec l'environnement matériel et le rythme de vie: l'urbanisation, les déplacements, l'importance des mass media. Une deuxième source, et qui touche de près les enfants, est le milieu ou climat familial: difficultés matérielles, disputes fréquentes des parents entre eux ou avec les enfants, alcoolisme ou trouble psychique d'un membre de la famille (Rivolier, 1989). 
Une troisième source aussi importante concerne les changements importants de la vie. Les questionnaires d'événements existentiels tentent de dresser une liste plus ou moins exhaustive de ces changements importants qui peuvent surgir dans la vie de chacun, mais doivent cependant être différenciés selon l'âge. Parmi ces événements, on mentionne comme les plus importants le décès d'un parent, ou d'une personne proche, le divorce et la perte de son emploi.

On s'intéresse de plus en plus aujourd'hui aux conditions occupationnelles, à savoir le travail, comme source de stress. Certaines professions engendrent un stress particulier lié à la surcharge, aux difficultés interpersonnelles rencontrées, à la pression suscitée par la concurrence, ou même à la peur de perdre son emploi si l'on n'est pas assez performant. À ce stress appelé burn out en anglais, on ajoute aussi de nos jours le stress pouvant venir du mobbing, à savoir le fait qu'un employé peut, sur son lieu de travail, être victime de harcèlement moral, de la part d'un supérieur hiérarchique ou de collègues. Le stress au travail a aussi des répercussions importantes sur la vie familiale et sur la qualité de la relation entre parents et enfants.

On peut encore citer comme sources de stress les causes situationnelles, comme le fait d'être hospitalisé, d'être emprisonné ou de se trouver dans des situations exceptionnelles (être au chômage, être réfugié).

Le stress touche tous les âges de la vie. Selon Compas (1987), la problématique de la séparation et de l'attachement au premier âge de la vie peut déjà être considérée dans l'optique du stress. Puis viennent toute une série de situations qui peuvent affecter ou non le développement harmonieux des enfants. Pour les enfants d'âge préscolaire, il y a le début d'un séjour en crêche ${ }^{3}$ ou le changement de crêche, la naissance d'un nouvel enfant ou l'arrivée d'un nouveau membre de la famille, la séparation d'un parent, le fait de recevoir une punition, l'entraînement à la propreté. Les jeunes enfants peuvent aussi être préoccupés par la peur d'être abandonnés. Ce qui est étrange ou étranger peut aussi stresser certains d'entre eux.

Pour les enfants d'âge scolaire, les situations stressantes et les tracas quotidiens ne manquent pas: avoir un nom peu commun, passer un examen, être poussé à avoir de bonnes notes, se sentir trop petit, trop gros, malhabile, se faire de nouveaux amis, être jaloux, avoir des disputes avec ses frères et sœurs ou ses parents, ne pas s'entendre avec le maître ou la maîtresse d'école, se sentir exclu d'un groupe de pairs, etc.

3. Au sens de garderie en Amérique du Nord. 
Le stress scolaire est souvent celui que les enfants et les parents considèrent comme le plus important durant la dernière période de $l^{\prime}$ enfance et à l'adolescence. Ce stress n'est cependant pas uniforme, il peut prendre différents aspects. Comme l'a souligné Maria Tyszkowa (1990), on peut parler du stress pour des tâches trop difficiles, que ce soit à cause de la surcharge (trop d'examens ou de devoirs à préparer) ou la peur d'avoir de mauvaises notes. Mais le stress peut aussi provenir de situations interpersonnelles difficiles : être socialement exposé (évaluation devant la classe), avoir des conflits avec l'enseignant ou d'autres élèves ou encore être forcé de se comporter de manière particulière pour répondre aux normes de l'école ou des pairs.

Avec l'arrivée de la puberté, aux stresseurs précédents viendra s'ajouter les inquiétudes propres à la puberté, et en particulier celles qui concernent les changements corporels et les relations avec les parents et les pairs. C'est précisément l'impact de ces différents stresseurs sur la santé physique que nous avons étudiés dans notre recherche sur les événements existentiels et les tracas quotidiens.

\section{LE STRESS DES ÉVÉNEMENTS EXISTENTIELS}

Pour étudier l'effet du stress sur la santé, on a fait des recherches d'abord sur les événements existentiels, ou stresseurs majeurs, et ensuite sur les tracas quotidiens, ou stresseurs mineurs. Les deux types de stresseurs ne sont pas toujours faciles à distinguer et les instruments utilisés pour les mesurer contiennent parfois des items pouvant relever des deux catégories. En outre ils sont souvent liés entre eux, comme la puberté, qui est un événement existentiel (pas négatif en soi) pouvant engendrer des tracas quotidiens.

Les études sur les événements stressants durant l'enfance et l'adolescence sont moins nombreuses que celles sur les adultes et ont été menées suivant la même optique. Dans les études portant sur les événements existentiels vécus par les enfants et les adolescents, on s'est largement inspiré de la procédure utilisée pour les adultes. On trouve dans Johnson (1986) une présentation historique des différentes échelles créées pour étudier les événements stressants chez les enfants et les adolescents.

C'est Coddington qui fut le premier à élaborer un questionnaire, le Life Event Record, dont le contenu avait été déterminé par un groupe d'enseignants, de pédiatres et de psychologues (Coddington, 1972a, 1972b), selon une méthode semblable à celle de Holmes et Rahe (1967), 
à savoir l'attribution, pour chaque item, d'un score représentant la quantité de réajustement psychosocial à fournir par les sujets après l'événement en question. Le projet de Coddington est abondamment cité et a inspiré les autres tentatives. On a cependant reproché à son questionnaire de ne pas distinguer événements positifs et événements négatifs, de ne pas inclure tous les événements que les enfants et les adolescents peuvent vivre et, enfin, de ne tenir compte que de l'opinion des adultes sur le caractère stressant des événements. C'est pourquoi, en 1980, Johnson et McCutcheon (1980) mirent au point un questionnaire, le Life Event Checklist, qui incluait des événements contrôlables et non contrôlables. Pour chaque événement, le sujet devait dire s'il était bon ou mauvais et indiquer, sur une échelle de 0 à $3, l^{\prime}$ importance de l'effet qu'il avait eu sur sa vie.

En 1985, Swearingen et Cohen proposèrent un questionnaire semblable au précédent; après plusieurs recherches, ils lui donnèrent une forme simplifiée qu'ils appelèrent JHLES (Junior High-School Life Experiences Survey). Les auteurs arrivèrent en effet à la conclusion que le meilleur indicateur des événements existentiels était la somme des événements négatifs. On laisse donc seulement au sujet la possibilité de déterminer pour chaque événement s'il est positif, négatif ou neutre. Néanmoins, les auteurs font dans leurs analyses la distinction entre événements contrôlables et événements non contrôlables, et entre événements désirables et événements non désirables, distinction basée sur le jugement d'un nombre important de psychologues (Swearingen et Cohen, 1985; Plancherel, Núñez, Bolognini, Leidi et Bettschart, 1992).

Après avoir choisi, pour étudier le stress, les événements de vie critiques, on s'est intéressé depuis quelques années aux tracas quotidiens comme facteurs de stress pouvant influencer de façon tout aussi importante le bien-être psychique ou physique. Dans le langage courant, on utilise souvent le terme de "tracas" pour désigner les soucis vécus par tout un chacun de façon plus ou moins continue et dans une proportion plus ou moins importante. Pour parler des tracas quotidiens, les Anglosaxons utilisent le terme de hassles alors que les Germanophones parlent plutôt de Mikrostressoren. Le concept de tracas partage avec celui de stress un caractère polysémique: on parle en effet de tracas aussi bien au plan subjectif (se tracasser) qu'au plan objectif (les tracas de la ville). De même, le stress est utilisé soit comme synonyme de stresseur objectif (une activité stressante), soit comme un état subjectif (être stressé). Les dictionnaires reprennent cette double acception. On trouve dans Le Petit Robert la définition suivante: "Dans son acception moderne: souci ou dérangement causé par des préoccupations d'ordre 
matériel, harcelantes, sinon graves. "Le tracas est ainsi apparenté au souci et les deux termes sont considérés parfois comme synonymes, comme en témoigne le Larousse des synonymes qui au mot "souci" donne les synonymes: tourment, angoisse, cauchemar, tracas.

Il n'est pas facile de dire dans quelles circonstances les termes strains (tensions) et hassles (tracas) sont entrés pour la première fois comme concepts opérationnalisés dans la recherche psychologique. C'est Lazarus qui s'intéressa le premier à l'importance des stresseurs mineurs de la vie quotidienne (Lazarus et Cohen, 1980; Lazarus, Kanner et Folkman, 1980). On considère d'ailleurs que le Berkeley Stress and Coping Project de Lazarus, DeLongis et Kanner, en 1977, fut à l'origine des autres recherches sur les tracas quotidiens, comme stresseurs mineurs par opposition aux événements existentiels qui sont des stresseurs majeurs (Kanner, Coyne, Schaefer et Lazarus, 1981; DeLongis, Coyne, Dakof, Folkman et Lazarus, 1982).

L'origine de l'utilisation du concept de strain est moins claire. Utilisé abondamment dans la recherche de laboratoire sur les animaux (souris), le mot strain est synonyme de stress dans ce contexte. Pearlin a souligné, dans les années 1980, l'importance de distinguer les stresseurs chroniques des stresseurs ponctuels. Bien qu'ils n'en soient qu'un sousensemble, ce sont les "role strains ", ou tensions chroniques liées à un rôle particulier (comme être enfant ou adolescent, être garçon ou fille, étudiant ou apprenti, fils/fille de), qui sont le plus souvent mentionnés (Pearlin, 1983; Bird et Harris 1990). Les tracas étant des stresseurs mineurs ponctuels se caractérisant par leur répétition, il est parfois difficile de les distinguer des "strains" ou tensions chroniques.

On a pu constater l'effet des événements existentiels sur la santé, mais cet effet est relativement modeste, aussi bien chez les adultes que chez les enfants et les adolescents (Cohen et Edwards, 1989; Johnson, 1986; Plancherel et al., 1992 ; Plancherel, Bettschart, Bolognini, Dumont et Halfon, 1997). On a donc pensé que ce phénomène venait en partie des ressources de chaque sujet (ou "facteurs protecteurs"), ce qui a engendré l'hypothèse de l'effet "buffer" ou "effet tampon ", mais aussi que les tracas quotidiens pouvaient être un meilleur prédicteur des problèmes d'ajustement et donc des symptômes psychiques. Lazarus et ses collègues ont ainsi avancé l'hypothèse que des événements stressants relativement mineurs de la vie de tous les jours pouvaient avoir un rôle essentiel. McLean (1980) avait quant à lui suggéré que l'on avait peut-être minimisé l'importance des événements quotidiens de la vie comme stresseurs du fait qu'ils sont familiers et que l'unité de stress est relativement petite. 
Il n'est pas toujours facile de distinguer un stresseur majeur et un stresseur mineur, car la perception que le sujet en fait est déterminante pour l'établissement de cette distinction. On peut cependant différencier objectivement les événements existentiels selon qu'ils sont normatifs ou non-normatifs. Les événements normatifs sont ceux que chaque sujet doit vivre. Durant la période qui nous intéresse, il s'agira du développement de la maturation biologique, de l'âge chronologique, du changement de niveau scolaire, ainsi que du développement cognitif. Par contre, on mettra dans les événements non-normatifs les événements historiques touchant la société en général (une guerre, une dépression économique), ainsi que les événements existentiels liés à l'histoire de chaque individu (séparation des parents, deuil).

Le lien entre tracas et événements existentiels peut prendre différentes formes. On peut penser à deux effets indépendants s'additionnant pour créer le stress. Mais on peut penser aussi à un rôle médiateur des tracas entre les événements existentiels majeurs et la santé. C'est l'idée que Kanner, Feldman, Weinberger et Ford (1987) ont émise. Par contre, pour Rutter et Standberg (1992), plusieurs hypothèses peuvent être envisagées: 1) des tracas amènent peu à peu à un événement existentiel (une discorde familiale peut se terminer par un divorce); 2) des tracas rendent le sujet plus vulnérable pour faire face aux événements existentiels ; 3) un événement existentiel peut engendrer des tracas (le fait de devoir porter des lunettes peut provoquer la moquerie de la part de certains camarades).

\section{LES MALADIES DU STRESS}

On a pu mettre en lumière plusieurs maladies qui seraient directement dépendantes du stress. On distingue d'habitude les maladies de l'adaptation et les maladie de l'esprit ou du comportement.

Pour Selye, les maladies de l'adaptation traduisent l'antagonisme entre les minéralocorticoïdess et les glucocorticoïdes, mais c'est surtout l'excès de minéralocorticoïdes qui conditionne ce type de maladie (Lôo et Lôo, 1995). Les principales maladies de l'adaptation sont l'asthme, l'ulcère à l'estomac, l'hypertension artérielle, certaines formes de rhumatisme, certains diabètes. On y ajoute aussi les maladies psychosomatiques qui sont des affections de pathologie générale relevant en partie ou en totalité de facteurs psychologiques conscients ou inconscients, et qui sont donc une conséquence du stress émotionnel. On pense aussi que certains types de personnalités sont des terrains plus favorables à ce type de maladie, 
en particulier les sujets alexithymiques, sujets qui ont de la peine à ressentir, à nommer et à exprimer leurs émotions, mais aussi les sujets ayant des mécanismes de défense rigides, une anxiété marquée, une personnalité narcissique recherchant l'admiration d'autrui. Enfin, on trouve les maladies allergiques, ce qui découle directement du modèle immunologique du stress.

Dans les maladies de l'esprit ou du comportement, on inclut la plupart du temps l'angoisse, sentiment d'insécurité, appréhension confuse, peur sans objet, qui peut prendre l'aspect soit de l'anxiété généralisée, soit de l'attaque d'angoisse ou de panique (phobie) et la dépression, modification de l'humeur qui s'accompagne d'inhibition sur le plan intellectuel, affectif, sexuel (Rivolier, 1989).

On parle parfois de syndromes spécifiques, dans le cas où le stress a un caractère exceptionnel et dramatique, comme un incendie, un tremblement de terre, une situation de guerre, un accident, un viol, une prise d'otage, ou par suite d'un isolement ou d'un confinement. Les symptômes du stress post-traumatique se manifestent dans le fait de revivre l'événement en pensée de manière persistante et d'éviter les situations qui rappellent l'événement. Chez les enfants, cela se manifestera par des troubles du sommeil ou des cauchemars, des changements dans l'appétit, des comportements régressifs (sucer son pouce), agressifs ou hyperactifs, mais aussi dépendants et manifestant un attachement excessif. Chez les adolescents, on trouvera un manque de concentration, une recherche d'isolement et de repli sur soi, une humeur dépressive et des idées suicidaires, mais aussi des comportements agressifs. La recherche dans le domaine du stress chez les enfants s'est particulièrement intéressée au stress post-traumatique (SPT), après des catastrophes naturelles ou des guerres (Pfeffer et al., 1996). On a pu constater également que des abus ou de la négligence pouvaient être à l'origine d'un trouble de type SPT. Dans une recherche portant sur plus de 1000 enfants, plus d'un tiers des sujets qui avaient été victimes d'abus physiques ou sexuels ou qu'on avait négligés remplissaient les critères DSM-III-R du trouble SPT soit actuel, soit “ lifetime» (Widom, 1999).

\section{DONNÉES DE RECHERCHE}

Avec les instruments qu'on vient de mentionner, ou par des méthodes similaires, de nombreuses recherches ont trouvé une relation entre le stress occasionné par des stressseurs majeurs ou mineurs et des problèmes de santé physique ou psychique. On peut lire chez Johnson (1986) ou 
Compas (1987) l'état de la question à cette date. On a pu trouver une relation entre le stress et les douleurs abdominales récurrentes sans cause physique bien déterminée (Greene, Walker, Hickson et Thompson, 1985; Hodges, Kline, Barbero et Flanery, 1984). Des études ont aussi montré une relation entre le stress et les maladies respiratoires chez des enfants dont la moyenne d'âge était de 4,3 ans (Boyce, Jensen, Cassell, Collier, Smith et Raimey, 1973), et entre le stress et l'apparition d'un cancer (Jacobs et Charles, 1980). D'autres études se sont intéressées aux maladies chroniques chez les enfants: asthme, diabète, fibrose kystique (Bedell, Giordani, Amour, Tavormina et Boll, 1977).

Mais la plupart des études se sont intéressées aux relations entre stress et troubles psychiques. Certaines n'ont pris en compte qu'un niveau global d'ajustement, d'autres se sont centrées sur des troubles spécifiques: performances scolaires, dépression, troubles alimentaires. Dans la recherche sur les événements de vie, on a mis en lumière dès le début un lien entre stress et dépression (Paykel, Myers, Dienelt, Klerman, Lindenthal et Pepper, 1969; Brown, Sklair, Harris et Birley, 1973). Mais cela était limité au domaine adulte. La recherche portant sur les enfants n'a pas donné les mêmes résultats. Ainsi, Steinhausen et Radtke (1986) ont comparé un groupe d'enfants ayant des troubles psychiques et un groupe d'enfants n'en ayant pas, et n'ont pas trouvé de grandes différences. Dans l'ensemble, la discrimination suivant la gravité des événements était plus révélatrice chez le groupe des sujets ayant des troubles du comportement, que chez le groupe ayant des troubles psychiques. Chez les enfants, les événements de vie ont donc plus d'influence sur les troubles du comportement, comme l'ont aussi démontré Bayley et Garralda (1987, 1990).

Les enfants fréquentant des cliniques psychiatriques ont souvent vécu plus d'événements critiques, dont les principaux ont un lien avec le fonctionnement psychosocial des parents ou des enfants eux-mêmes (Jensen, Richters, Ussetry, Bloedau et David, 1991). Auerbach, Lerner, Barasch et Palti (1992), par exemple, ont trouvé que les événements existentiels liés à la mère étaient des prédicteurs du succès scolaire ainsi que des problèmes de comportement aussi bien chez les filles que chez les garçons. Beaucoup de recherches ont été menées sur les conséquences du divorce sur le développement des enfants (Wallenstein, 1985; Kaslow, 1990). Cependant, les mécanismes à l'œuvre lors de tels événements sont encore mal connus. Ainsi Rutter et Standberg (1992) insistent sur la connexion entre événements et tensions chroniques. Dans le cas du divorce, des tensions chroniques amènent peu à peu à un événement critique, comme une discorde familiale peut se terminer par un divorce. 
Dans d'autres recherches, on a cependant pu mettre en relation le stress des événements existentiels avec des troubles comme l'anxiété et la dépression. Pour ce qui est de la dépression, Garber et Hilsman (1992) présentent un inventaire exhaustif de toutes les recherches faites en indiquant l'âge des sujets, la taille de l'échantillon, la mesure de la dépression (la plupart du temps le $\mathrm{CDI}^{4}$ ), le type de stresseur (mineur ou majeur) et finalement le résultat sous forme de corrélation. On distingue aussi les recherches faites avec un échantillon tout-venant et les recherches comparant un échantillon clinique à un échantillon non clinique. Les résultats de ces 25 études montrent qu'il y a un lien entre le stress et la dépression mais les corrélations varient entre ,20 et ,50. On conclut généralement qu'il y a bien un certain support à l'hypothèse que le stress cause des problèmes d'ajustement psychologique. Mais on doit être prudent pour interpréter les résultats. La plupart des recherches sont rétrospectives, et l'on peut toujours supposer une confusion entre symptômes et événements. Peu de recherches citées par Garber et Hilsman (1992) étudient l'effet tampon de certains facteurs protecteurs comme le support social.

Dans le cas des enfants, on peut dire qu'il existe un lien entre le stress des événements existentiels et des tracas quotidiens, et la dépression, les troubles de la conduites, les troubles du déficit d'attention (Goodyer, 1994, 1996; Goodyer, Kolvin et Gatzanis, 1985; Goodyer, Wright et Altham, 1988; Goodyer, Wright et Altham, 1990). Mais il est souvent difficile de ne s'en tenir qu'à des catégories diagnostiques isolées. On sait qu'il y a une importante comorbidité des troubles dépressifs et non dépressifs, et que ces derniers sont souvent masqués par des troubles de la conduite et du déficit d'attention (Bird, Canino, Rubio-Stipec, Gould, Ribera, Sesman, Woodbury, Huertas-Goldman, Pagan, Sanchez-Lacay et Moscoso, 1988). Cela est particulièrement vrai pour les garçons.

À partir de l'observation et des données des recherches, et dans un but de prévention, on a tenté de dresser une liste des signes qui pouvaient indiquer chez les enfants une perturbation due au stress. Il y a beaucoup de variation d'une liste à l'autre, mais, en général, on retrouve les signes suivants: troubles du sommeil, troubles de la conduite, comportement dépendant, manque de concentration, irritabilité, régression (sucer son pouce). À cela s'ajoutent des signes qui peuvent être des troubles physiques comme des maux de tête ou d'estomac sans cause apparente. Et si le stress est survenu par suite d'un événement traumatisant, comme un

4. Child Depression Inventory de Kovacs (1980). 
désastre, on trouve ce qu'on appelle le profil des troubles du stress posttraumatique: événement revécu dans les pensées et les rêves, évitement des stimuli associés au traumatisme, symptômes traduisant une hyperactivité neurovégétative (réaction de sursaut exagérée, accès de colère, hypervigilance).

\section{CONCLUSION}

Tout le monde s'accorde aujourd'hui pour voir dans le stress une source importante de troubles tant physiques que psychiques. Il est bon cependant de se rappeler que le stress est essentiel à l'homme, car c'est une réaction d'adaptation aux contraintes de l'environnement. Un environnement menaçant stimule nos systèmes d'éveil et de lutte: notre cerveau sécrète certaines molécules, cortisol et catécholamines, qui nous rendent plus aptes à lutter, mais aussi plus anxieux. Si les contraintes perdurent et sont plus graves, il devient plus difficile de faire face et l'on entre alors dans le pathologique. Le système d'adaptation va s'épuiser et apparaîtront alors des symptômes d'anxiété et de dépression. Les enfants sont eux aussi victimes du stress. Ils sont même plus vulnérables que les adultes, car plus dépendants, ils ne peuvent fuir ou s'isoler, et ils n'ont pas déjà forgé des armes pour se défendre contre le stress.

Les recherches portant sur le stress et son impact sur la santé des enfants sont confrontées aux mêmes difficultés que celles portant sur des populations d'adultes ou d'adolescents (Thoits, 1983). Pour ce qui est de la mesure de la variable dépendante (santé), on se trouve toujours confronté à la validité de la mesure, et aussi au problème du temps écoulé entre l'événement et l'éclosion du trouble. Le diagnostic est plus difficile chez les enfants et les jeunes adolescents du fait de l'importante comorbité qu'on trouve à cet âge, la dépression étant souvent masquée par les troubles du comportement. Quant à la mesure de la variable indépendante (événements existentiels ou tracas quotidiens), on se trouve aussi confronté à différents problèmes : problèmes liés au souvenir, au contenu des échelles, confusion entre stresseurs et troubles, certains pouvant être souvent des effets ou des symptômes des troubles. La hiérarchie de la causalité n'est pas toujours facile à déterminer, et c'est encore plus vrai pour les tracas quotidiens. Certains auteurs pensent qu'il est préférable de procéder par interview que par questionnaire autogéré; dans le cas des enfants, il est d'ailleurs recommandé d'interroger aussi les parents pour avoir une meilleure information sur les caractéristiques des stresseurs (Goodyer, 1994; Rutter et Standberg, 1992). 
On a aussi beaucoup déploré que les premières études sur le stress n'aient pas tenu compte de variables modératrices (facteurs de vulnérabilité ou facteurs protecteurs). Depuis, un important champ de recherche s'est développé pour prendre en compte ce phénomène. Si l'on consulte les bases de données, on ne trouve plus guère de recherches portant uniquement sur l'effet du stress sur la santé, l'accent étant mis sur les ressources des sujets : la façon de gérer le stress (coping), le support social, l'estime de soi, la résilience.

Compas (1987) soulignait déjà la nécessité, pour l'étude du stress chez les enfants et adolescents, de prendre en compte les relations avec l'environnement (familial en l'occurrence), et le tempérament, certains enfants étant plus sensibles au stress que d'autres. Enfin, il est nécessaire de tenir compte des caractéristiques cognitives et sociales liées au développement (par exemple auto-efficacité, locus of control, estime et concept de soi).

Un développement nouveau dans l'étude du stress, qu'il soit rattaché aux stresseurs mineurs ou majeurs, est la prise en compte du caractère social de ce phénomène. Cela répondrait au premier souhait de Compas (1987). Des recherches ont déjà été faites sur le stress dans les couples. On s'est intéressé depuis peu au stress familial. Il s'agit de tous les stresseurs vécus par des sujets dans la vie quotidienne et dans les interactions qu'ils ont avec les membres de leur famille. Cet aspect du stress est spécialement adapté aux enfants et aux jeunes adolescents, étant donné qu'une grande partie des stresseurs auxquels ils font face ont une relation avec leur milieu familial. Quoique l'analyse de ce stress soit encore à ses début, et que l'analyse des données soit plus complexe, la prise en compte de ce contexte familial sera un apport important et enrichissant aux précédentes recherches (Perrez, Plancherel et Ababkov, 2000). 



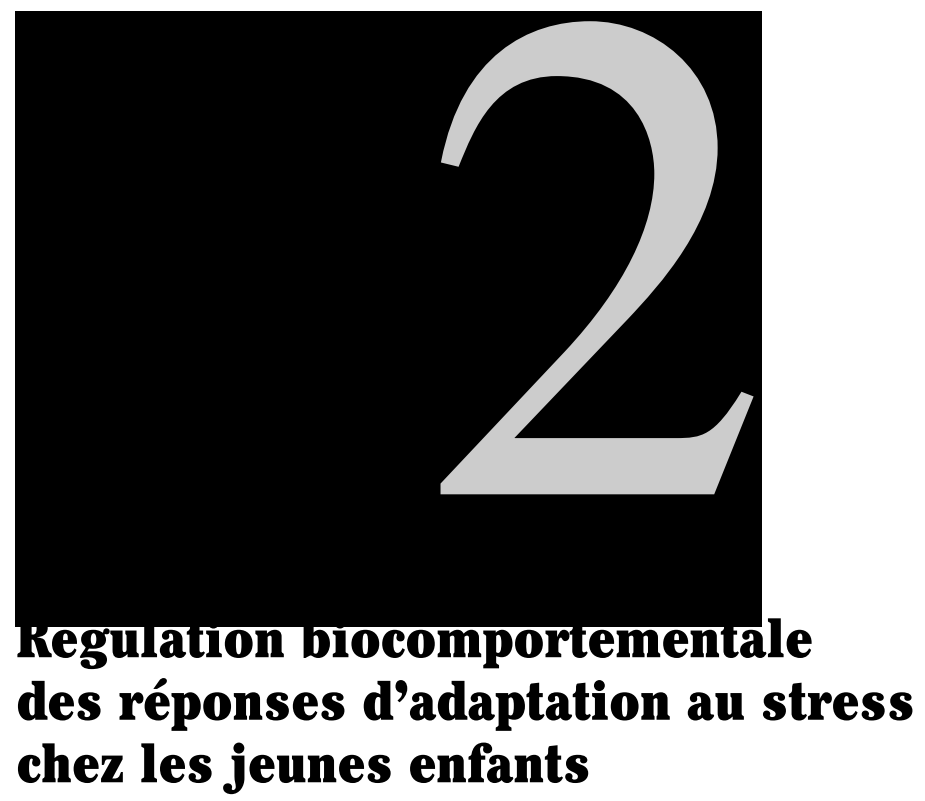

MARCEL TRUDEL

Université de Sherbrooke

Sherbrooke (Québec)

ÉLISABETH CHAUSSEGRos DE LÉRY

Université de Sherbrooke

Sherbrooke (Québec)

GUADALUPE PUENTES-NEUMAN

Université de Sherbrooke

Sherbrooke (Québec) 

$\mathrm{O}$ reconnaît de plus en plus que les processus d'adaptation individuelle sont le reflet de l'influence réciproque des caractéristiques de l'organisme et du contexte environnemental. Lorsque l'on veut estimer les capacités adaptatives de l'individu face à l'adversité, on se doit de considérer à la fois son répertoire comportemental, le processus de régulation physiologique et l'interaction des deux. Le présent chapitre dresse d'abord l'état de la recherche sur les processus physiologiques qui sont mobilisés lors de situations qui exigent une adaptation individuelle ou qui déclenchent le stress. Dans un tel contexte, nous tenterons également d'élucider la question de la régulation des systèmes physiologiques et comportementaux d'un point de vue interactif. Nous présenterons par la suite un bilan critique des travaux de recherche portant sur l'analyse de l'adaptation en groupe de pairs, qui représente pour les enfants un contexte social potentiellement stressant. Cette recension permettra de faire le point sur l'état de nos connaissances sur la relation entre l'adaptation comportementale et la régulation physiologique chez les jeunes enfants. Enfin, à ce bilan de la recherche, nous proposons un cadre théorique susceptible de mieux rendre compte de la conceptualisation qui est faite de la régulation biocomportementale.

Au cours du dernier quart de siècle, beaucoup de chercheurs se sont intéressés à l'étude de l'influence de la maturation et des expériences précoces sur le développement humain. Ces derniers se sont surtout orientés vers l'investigation des interactions entre les facteurs organiques et les facteurs environnementaux qui sous-tendent le développement de l'individu (Cairns, 1998; Lerner, 1984). Dans le sens des études reconnaissant une perspective dynamique au développement, Gottlieb (1973) affirme qu'il est de plus en plus difficile de comprendre le fonctionnement des mécanismes régulatoires liant le comportement et la physiologie en adoptant un déterminisme strictement génétique. La vision moderne en psychobiologie du développement ne peut non plus contourner l'investigation des processus physiologiques impliqués dans l'adaptation comportementale (Gottlieb, 1991). Ce point de vue doit nous conduire à découvrir la nature des liens entre les processus physiologiques et les processus 
comportementaux afin de mieux cerner les mécanismes dynamiques de régulation biocomportementale. Toutefois, l'approche psychobiologique du développement ne peut pas prétendre rendre compte de l'ensemble des modalités organiques impliquées dans l'adaptation sociale; elle doit plutôt favoriser l'étude des indices permettant de cerner les processus endogènes et exogènes qui orientent le développement individuel. Une telle approche nécessite une intégration interdisciplinaire pour en arriver à une compréhension nuancée du développement humain.

Sans vouloir alimenter l'éternel débat nature/culture, nous postulons que, chez les enfants, les séquences comportementales se manifestent sous la double contrainte de l'expérience préalable (par exemple, l'expérience sociale auprès des pairs) et de leurs états physiologiques. Il importe donc de comprendre comment s'enchaînent les réactions comportementales, ou de coping, des partenaires sociaux en fonction, d'une part, du contexte écologique où ils sont placés et, d'autre part, de leur état physiologique respectif.

\section{PERSPECTIVE PHYSIOLOGIQUE DE L'ADAPTATION AU STRESS}

La notion de stress est née avec les travaux de Cannon (1935) en psychosomatique. Pour définir le phénomène observé, il emprunta le mot "stress" aux sciences physiques. Ainsi, à l'image des matériaux qui se déforment sous l'action des contraintes, l'individu présente des symptômes physiologiques sous l'effet de certains facteurs externes. Celui-ci propose que le stress psychologique et le stress émotionnel sont à l'origine de plusieurs problèmes physiologiques. À la même époque, Hans Selye (1956) observa également, au cours de ses travaux avec des rats auxquels il injectait un médicament, des réactions physiologiques inattendues, peu importe le produit injecté. Les animaux présentaient des ulcères d'estomac, des déficiences immunitaires et une hypertrophie des glandes surrénales. Pour Selye, ces animaux avaient un seul point en commun, les injections répétées. Pour vérifier son hypothèse, il fit subir aux rats différentes situations d'adversité: changement intense de température, agents pathogènes, toxines et bruit intense. Il put observer les mêmes réactions peu importe la nature des stimuli. Cette conception du stress est cependant remise en question puisque l'on reconnaît à l'heure actuelle que chez les humains les réactions à un même stresseur peuvent varier d'un individu à un autre et d'un contexte à l'autre (Carroll, 1992). Il est 
important de souligner, à cet égard, la contribution des travaux de Lazarus et Folkman (1984) qui ont testé la relation entre les composantes psychologique et physiologique du stress (voir Bruchon-Schweitzer et Dantzer, 1994). Ces chercheurs soulignent que l'individu doit d'abord percevoir et interpréter une situation comme stressante pour que se déclenche une réaction physiologique. Ce paradigme propose que le stress doit être considéré comme un processus de transaction entre l'individu et le milieu (Lazarus et Laumier, 1978) (pour plus de précisions sur ce modèle voir le chapitre 3). Il est clair que si l'on accepte cette définition on se doit de porter attention aussi bien aux conditions endogènes qu'aux conditions exogènes à l'organisme. On doit tenir compte des caractéristiques des événements environnementaux et psychologiques qui menacent l'intégrité ou le bien-être de l'individu, de l'interprétation qui est faite de ces événements, et des ajustements comportementaux et physiologiques auxquels procède l'individu pour rétablir son équilibre interne.

\section{LES MÉCANISMES PHYSIOLOGIQUES ASSOCIÉS AU STRESS}

Dans la vie quotidienne, différents mécanismes physiologiques sont impliqués dans le maintien d'un état homéostasique, ou d'équilibre interne. Lors d'un stress, extrême, inhabituel ou de longue durée, ces différents mécanismes ne peuvent suffire à la tâche. Dans ce cas, il se produit un ensemble de changements physiologiques qui perturbent le milieu interne normal. Un ensemble de réactions neurales, neuroendocriniennes, métaboliques et comportementales se mobilisent afin d'augmenter les chances de l'organisme de répondre adéquatement au stimulus perçu comme stressant. Mais quel est le mécanisme par lequel l'organisme rétablit cet équilibre physiologique?

Lorsque le cerveau, en l'occurrence l'aire hypothalamique, perçoit un agent de stress, il transmet l'information par les voies nerveuses et hormonales. Deux systèmes neuroendocriniens réagissent au stress et influencent éventuellement les réponses émotionnelles et cognitives de l'individu. Le premier système s'active rapidement et implique l'activation de la glande médullosurrénale qui sécrète les catécholamines comme l'adrénaline et la noradrénaline. Cette glande est liée à l'activation du système nerveux sympathique. On observe des concentrations élevées d'adrénaline et de noradrénaline dans le sang et l'urine à la fois avant et pendant les activités dites stressantes. C'est ce qui ressort des études en psychobiologie du stress qui ont examiné les réactions endocriniennes de 
parachutistes dans des situations d'entraînement (voir Ursin, Baade et Levine, 1978). Cette activité hormonale s'accompagne également d'une régulation du système nerveux végétatif sympathique et parasympathique commandant notamment l'activation du système cardiovasculaire. Ainsi, une augmentation du taux de concentration de l'adrénaline et de la noradrénaline contribue à accélérer le rythme cardiaque afin d'aider le cœur dans sa fonction d'oxygénation des tissus de l'organisme. Les résultats des recherches sur la réactivité des fonctions cardiovasculaires qui sont associées au stress suggèrent qu'une forte réactivité cardiaque correspondrait à une prédisposition individuelle, constituant elle-même un facteur de risque, susceptible de prédire l'état de santé et de bien-être de l'individu (Boyce, Alkon, Tschann, Chesney et Alpert, 1995; Jemerin et Boyce, 1992; Lewis, Thomas et Worobey, 1990).

Le deuxième système stimulé par le stress est l'axe impliquant l'hypothalamus, la glande pituitaire et la glande surrénale (HPS) qui permet à l'organisme de continuer à combattre un agent stressant, même après la disparition des effets immédiats. C'est ce dernier aspect qui a surtout retenu l'attention des chercheurs en psychobiologie du stress. L'activation de l'axe HPS génère à la fin du processus la sécrétion des glucocorticoïdes, principalement le cortisol chez l'homme et le singe, et la corticostérone chez les rongeurs. La concentration de cortisol dans la salive, le sang et l'urine est un élément révélateur de l'activité combinée de l'hypophyse et des corticosurrénales (voir Rosenzweig et Leiman, 1989). L'activité de l'axe HPS implique d'abord l'activation des noyaux hypothalamiques, qui libèrent la corticolibérine $(\mathrm{CRH})$ dans le sang (voir le processus de régulation à la figure en annexe). La libération de la CRH stimule par la suite l'aire hypophysaire qui, par l'action de la glande pituitaire, va secréter l'ACTH (1'hormone corticotrope). C'est cette dernière hormone qui active et contrôle la libération des hormones des corticosurrénales dont le cortisol. Lors d'un stress, la régulation de la sécrétion de la CRH, de l'ACTH et du cortisol est effectuée selon un processus de rétroaction négative. Ainsi, l'atteinte d'un certain taux de cortisol dans le sang déclenche une commande contraire à celle provoquée par le stimulus initial en ordonnant l'inhibition de l'activité de l'hypothalamus et de l'hypophyse et, par conséquent, de la production de CRH et d'ACTH. Ce processus de régulation hormonale va empêcher une activation prolongée et inutile du système HPS; dans le cas contraire, des signes cliniques de dysfonctionnement peuvent apparaître, comme une fatigue excessive et permanente faisant que le moindre assaut provenant de l'environnement deviendra insurmontable. L'activité de cet axe joue plusieurs rôles dans la résistance au stress: augmenter le taux de glucose produisant l'énergie nécessaire à l'action, réprimer des fonctions 
non immédiatement essentielles telles que la reproduction et la digestion, retarder la croissance de l'enfant (O'Leary, 1990), affecter le système immunitaire (Walker, Bodnar, Forget, Toufexix et Trottier, 1997) et, par son action sur le système limbique, perturber la régulation des émotions, l'apprentissage et la mémoire (Gunnar, 1992).

En général, ces réactions physiologiques au stress sont interprétées comme des mécanismes essentiels à l'adaptation aux changements. Lorsque l'agent du stress est de nature psychologique, les processus mis en œuvre pour assurer l'équilibre interne sont similaires. Ainsi, le simple fait d'exposer un individu à un environnement nouveau suffit pour déclencher une activation marquée du système endocrinien (Levine, 1993).

La production de base du cortisol suit un rythme nycthéméral (1'alternance sommeil/éveil) et un rythme circadien (une période de 24 heures entre deux pics de sécrétion). La sécrétion de cortisol se fait également selon un rythme ultradien, c'est-à-dire que la sécrétion endocrinienne est pulsative. Un message du système limbique sert à accorder les rythmes nycthéméral et circadien avec les activités quotidiennes normales comme se reposer et se nourrir. Chez les adultes qui ont un cycle normal jour/nuit et qui dorment des heures régulières, le pic du cortisol de base se produit durant la dernière heure de la nuit (après trois à cinq heures de sommeil). Le niveau élevé du matin aide à rendre l'énergie disponible pour l'action. Ce haut niveau décline fortement durant les premières heures après le réveil et plus graduellement par la suite. Chez les enfants, le système HPS atteint rapidement son état de maturité puisque, déjà entre le deuxième et le troisième mois, on observe l'établissement du rythme circadien dans la sécrétion du cortisol (Mantagos, Moustogiannis et Vegenakis, 1998; Price, Close et Fielding, 1983).

Dans des conditions normales, une grande part du cortisol se lie à une protéine, et le reste demeure libre dans le sang. Cette fraction libre est biologiquement active et génératrice d'énergie (glucose) (Gunnar, 1992). Le cortisol se retrouve également dans 1'urine et la salive. Dans l'urine, il est à la fois sous forme libre et liée à une protéine tandis que dans la salive, il est uniquement sous forme libre (Gunnar, 1992). Comme le cortisol est présent dans le sang, l'urine et la salive, ces liquides organiques peuvent être analysés pour évaluer le stress et l'état de santé de l'enfant. La technique d'échantillonnage plasmatique risque cependant de créer chez ce dernier un sentiment d'intrusion, donc de causer du stress et conséquemment d'altérer l'activité de l'axe HPS (Landon, Smith, Perry et Al-Ansari, 1982). L'utilisation de la salive se révèle donc un protocole très approprié puisqu'on y retrouve du cortisol à l'état libre (Aardal-Erksson, Karlberg, 
Holm, 1998; Schmidt, 1998). Cette procédure d'évaluation est également moins intrusive pour les personnes et plus particulièrement pour les enfants (Francis, Walker, Riad-Fahmy, Hughes, Murphy et Gray, 1987; Riad-Fahmy, Read et Hughes, 1983 ; Riad-Fahmy, Read, Walker et Griffiths, 1982; Woolston, Gianfredi, Gertner, Paugus et Mason, 1983). On peut effectuer le prélèvement à la maison sans personnel spécialisé (Kahn, Rubinow, Davis, Kling et Post, 1988). Sous l'action du stress, le cortisol salivaire prend de 10 à 15 minutes à produire un effet et de 20 à 30 minutes à atteindre son niveau maximum de sécrétion. Cette technique d'échantillonnage fournit donc de nouveaux moyens à l'étude des réactions corticosurrénales au stress quotidien.

\section{LA RÉGULATION PHYSIOLOGIQUE ASSOCIÉE À L'ADAPTATION COMPORTEMENTALE}

Il est indéniable que l'avancement accéléré des techniques de recherche en neuroscience a fortement contribué au regain d'intérêt de la psychobiologie du développement pour l'étude des processus intégrant les phénomènes neurophysiologiques et comportementaux associés à l'adaptation individuelle (Granger, Stansbury et Henker, 1994). Ainsi, l'accessibilité d'une méthodologie moins intrusive pour obtenir des indices de variation hormonale reflétant le stress physiologique ${ }^{1}$ a été déterminante dans le développement de nouveaux modèles psychobiologiques du comportement. Malheureusement, ces techniques de mesures physiologiques n'ayant vu le jour que très récemment, nous ne disposons pas à l'heure actuelle d'une information suffisante pour comprendre les mécanismes suivant lesquels les différentes réactions au stress sont liées aux divers engagements comportementaux. Sans nier l'importance des autres fonctions biologiques associées au stress, il semble de plus en plus évident cependant que la production de stéroïdes comme le cortisol fait du système endocrinien le principal agent de la relation entre les effets de l'environnement, l'adaptation physiologique et le comportement.

Dans les premiers travaux qui se sont intéressés à établir la relation entre le stress physiologique et le fonctionnement comportemental, on doit souligner la contribution de plusieurs recherches en primatologie.

1. Nous utiliserons de façon interchangeable les notions de stress physiologique et de stress biologique pour désigner la réponse physique d'un organisme à un stimulus stressant. 
Les études effectuées auprès des primates non humains ont notamment démontré que le statut de dominance sociale, indexé par les gains observés lors de situations de conflit, est directement lié à l'activation du système corticosurrénal (Manogue, Leshner et Candland, 1975). Ainsi, le niveau de concentration du cortisol plasmatique sécrété par les singes dominants en groupe stable est plus faible que celui de leurs partenaires subordonnés (Gust, Gordon, Hambright et Wilson, 1993). Cette tendance est également observée chez les groupes de singes en formation lorsque les relations interpersonnelles ne sont pas encore stabilisées (Chamove et Bowman, 1978; Scallet, Suomi et Bowman, 1981). Sapolsky $(1982,1990)$ rapporte quant à lui des résultats supportant la conception d'une régulation systémique des systèmes physiologiques et comportementaux. En effet, ses travaux font ressortir des corrélations négatives entre les changements longitudinaux observés dans le statut social des singes et la régulation dans les réponses endocriniennes; en l'occurrence, on observe chez le singe dominant qui a perdu son statut social une adaptation physiologique se traduisant par une augmentation du taux de concentration du cortisol. Ces résultats suggèrent la présence d'une forte relation entre les régulations comportementale et hormonale. La hiérarchie de ces effets n'est cependant pas suffisamment documentée pour dégager les mécanismes en cause (voir Sapolsky et Ray, 1989).

De l'ensemble des premiers travaux reliant l'adaptation physiologique et l'adaptation comportementale, il ressort trois courants théoriques expliquant les mécanismes d'interaction de ces deux systèmes. Le premier courant considère que la régulation biocomportementale est fortement déterminée par les prédispositions génétiques qui établissent les assises sur lesquelles l'environnement peut éventuellement jouer son rôle. Les résultats des travaux expérimentaux de Michael Meaney auprès de très jeunes rats démontrent que le stress génère une activation importante de glucocorticoïdes surrénaux qui vont affecter le développement de 1'hippocampe et du cortex cérébral. À plus long terme, une forte sécrétion de glucocorticoïdes contribuerait à freiner la croissance de l'individu. Ces travaux ont également mis en lumière les effets associés à la séparation mère-enfant, en indiquant que le comportement de léchage de la mère du jeune nourrisson va atténuer chez ce dernier l'activité des gênes responsables de la production de corticolibérine (CRH) et de glucocorticoïde (Meaney, Aitken, Bhatnager, van Berkel et Salpolsky, 1988 ; Meaney, Aitken, Viau, Sharma et Sarrieau, 1989; Benes, 1994). 
Le second courant postule une association entre l'état émotionnel et la régulation du cortisol (Mason, 1959, 1968). Ce modèle prédit que les indices associés à l'état de détresse du très jeune enfant sont directement liés à l'activation du système HPS (Gunnar, Connors, Isensee et Wall, 1988). Pour Tennes et Mason (1982), l'activité du système corticosurrénal et ses liens avec les systèmes émotifs sont le premier déterminant de l'activité endocrinienne du jeune enfant. Dès les premiers jours de la vie, les changements de comportement sont liés aux changements de l'activité corticosurrénale. Les résultats des premières études de Gunnar et ses collègues vont dans ce sens (Gunnar, Fish, Korsvik et Donhowe, 1981; Gunnar, Fish et Malone, 1984), en notant une relation significative entre le taux de cortisol et les comportements de détresse chez les nouveaunés ayant subi une circoncision. Cependant, cette association disparaît chez les enfants qui réussissent à se calmer au cours de cette situation anxiogène. Ces premières observations incitent à penser que si l'on répète l'action d'un stimulus négatif, on aura, à chaque fois, une hausse de cortisol. Toutefois, lors d'études auprès d'enfants subissant un stress répété comme des sessions de vaccination, on note que cet effet sur le cortisol s'est substantiellement atténué à la seconde session (Gunnar et al., 1988).

Gunnar (1989) a également observé ce phénomène auprès d'enfants âgés de 11 mois à 8 ans souffrant de phénylcétonurie (maladie héréditaire du métabolisme d'un acide aminé). Ces enfants doivent régulièrement, au cours de leur traitement, subir des prises de sang. Dans cette étude, on a observé le comportement de l'enfant et mesuré le taux de cortisol avant et après le traitement. Chez les enfants âgés de 5 ans ou moins, on observe une diminution des comportements de détresse (pleurs) et une augmentation du taux de cortisol. Les auteurs en déduisent que les enfants de cet âge n'ont pas encore développé des stratégies d'autocontrôle suffisantes pour maîtriser la situation (ce qui explique le taux de cortisol élevé). Ce n'est plus le cas à 6 ans, puisqu'on observe des taux de cortisol et de pleurs qui sont faibles. Dans l'ensemble, ces résultats pourraient suggérer que l'émotion négative n'est pas suffisante pour activer le système HPS (Gunnar, 1989).

Le troisième courant propose quant à lui que les réponses du système comportemental et du système endocrinien face à une situation de stress peuvent s'activer simultanément ou de façon indépendante (Levine, Coe et Wiener, 1989; Levine, Wiener, Coe, Bayart et Hayashi, 1987). On intoduit le concept de coping, ou de disponibilité d'un répertoire comportemental pour gérer l'adversité. On postule que la réponse corticosurrénale face à une situation de stress sera atténuée chez les enfants qui 
témoignent de stratégies adaptées dans leur organisation comportementale (le contrôle moteur et les processus d'attention ou d'orientation). Pour tester ce modèle auprès des nourrissons, Spangler et Scheubeck (1993) utilisent une procédure à deux volets: d'abord l'examen des effets associés à l'utilisation du protocole d'évaluation de Brazelton, qui sont évalués à deux reprises (Neonatal Behavioral Assessment Scale, NBAS) ${ }^{2}$ puis, l'estimation de l'impact de la mesure du rythme cardiaque à laquelle on procède durant l'une des sessions du NBAS.

Les auteurs rapportent que l'administration de la procédure NBAS ne suffit pas à déclencher une réponse du système corticosurrénal sauf chez un sous-groupe d'enfants. Par contre, l'application de la double procédure (NBAS et rythme cardiaque) fait ressortir un effet général où l'on observe une augmentation du taux de cortisol. À l'instar de Gunnar, Isensee et Fust (1987), Spangler et Scheubeck (1993) observent donc une sensibilité du système HPS aux effets d'une stimulation aversive modérée (NBAS) chez un sous-groupe d'enfants. Bref, plus la situation est aversive, plus le système corticosurrénal est susceptible de s'activer. Sur le plan comportemental, cette étude fait ressortir qu'un haut degré d'organisation de l'attention durant la tâche du NBAS est associé à un taux de base plus élevé de cortisol et vice-versa. Lorsqu'on mesure le cortisol durant la tâche, on observe une activation du système corticosurrénal uniquement chez les nouveau-nés qui manifestent une faible orientation au NBAS. Ces résultats confirment la validité du construit de coping suggérant que la réponse physiologique au stress sera modérée dans le cas où il y a disponibilité de stratégies comportementales adaptées à la situation. Selon cette perspective, l'organisation comportementale et l'activation endocrinienne doivent être considérées comme deux systèmes indépendants qui définissent le répertoire adaptatif de l'individu. En conclusion, lorsque le comportement suffit à assurer l'adaptation individuelle, l'activation endocrinienne $\mathrm{n}$ 'est pas nécessaire; conséquemment, on observera une forte relation entre les deux systèmes uniquement dans les situations de stress sévère ou imprévisible.

2. Le protocole de Brazelton (1984) permet d'évaluer l'organisation comportementale du nouveau-né. Le NBAS cible plusieurs aspects du comportement qui sont conceptuellement associés au tempérament de l'enfant. Ainsi, le test évalue l'intensité des réactions du nouveau-né ainsi que le niveau d'activité, de sociabilité et d'émotionnalité. 
De l'ensemble des travaux tentant de lier l'adaptation comportementale à la régulation endocrinienne, on doit conclure que l'on comprend encore difficilement les mécanismes qui associent les deux systèmes. D'abord, l'analyse des conditions à l'origine de l'activation du système corticosurrénal ne semble pas permettre de différencier clairement les types d'émotions déclenchées par les événements anxiogènes (Brown et Heninger, 1975). À ce problème, on doit ajouter notre manque de connaissances sur les contextes qui influencent l'adaptation comportementale et la régulation physiologique. Enfin, les mécanismes à l'origine de la régulation biocomportementale ne sont pas encore très bien cernés.

\section{LA RELATION ENTRE L'ADAPTATION COMPORTEMENTALE ET LA RÉGULATION PHYSIOLOGIQUE CHEZ LES ENFANTS EN GROUPE DE PAIRS}

Pour mieux comprendre le développement global de l'individu, on doit étudier les mécanismes biocomportementaux qui apparaissent au cours de l'enfance. D'entrée de jeu, on note peu de consensus quant aux facteurs psychologique et situationnel qui sont à l'origine de l'activation $\mathrm{du}$ système endocrinien. On doit supposer que l'axe HPS peut être mobilisé aussi bien par les facteurs personnels que par les facteurs contextuels, ou les deux. Plusieurs chercheurs ont commencé à étudier les différences individuelles dans l'influence réciproque de l'activité du système corticosurrénal et des processus sociaux sur le développement humain (Boyce et Jemerin, 1990; Chrousos et Gold, 1992). Ce point de vue soulève la possibilité que l'expérience sociale précoce, par exemple en groupe de pairs, joue un rôle important dans l'activation et la régulation de l'axe HPS face aux événements de l'environnement. Plus spécifiquement, l'interaction des caractéristiques de l'enfant et de l'environnement apparaît, pour plusieurs chercheurs, à l'origine du développement précoce de la régulation des émotions (Kopp, 1989; Thompson, 1991). Kagan, Reznick et Snidman $(1987,1988)$ observaient, par exemple, chez les enfants inhibés d'âge préscolaire, un rythme cardiaque faible et stable couplé avec une forte réactivité endocrinienne en situation d'interaction avec un adulte non familier. Ils en conclurent que cette inhibition comportementale demeurait stable et reflétait une prédisposition individuelle du tempérament. 


\section{L'ADAPTATION DANS UN NOUVEAU GROUPE DE PAIRS}

Au cours de la dernière décennie, quelques chercheurs ont abordé l'étude des effets de la nouveauté sociale ou du processus de formation des groupes de pairs sur l'adaptation comportementale et endocrinienne des jeunes enfants. Ainsi, les travaux réalisés par Trudel et ses collaborateurs (Jeunier, Trudel et Legendre, 1997; Legendre et Trudel, 1996, 1998 ; Trudel, 1998) illustrent la pertinence de considérer la régulation endocrinienne eu égard à ces processus d'intégration sociale chez les jeunes enfants, afin d'identifier les aspects comportementaux qui contribuent à réduire le stress et de tenir compte du rôle des variables contextuelles comme l'impact social des gestes. Le protocole d'étude prévoit l'observation de plusieurs groupes composés de six enfants non familiers de trois ans (trois garçons et trois filles). Cette recherche veut examiner la relation entre les niveaux de sécrétion du cortisol et les modes d'adaptation comportementale des enfants. Des prélèvements de salive ont été recueillis avant et après un épisode $\mathrm{d}^{\prime}$ interaction $\mathrm{d}^{\prime}$ une quinzaine de minutes ${ }^{3}$. L'influence du rythme circadien sur le système endocrinien est contrôlée en planifiant toutes les rencontres le matin et aux mêmes heures. Une première analyse du comportement individuel fait ressortir que les enfants ayant des comportements d'inhibition ont des taux de cortisol supérieurs aux autres (Legendre et Trudel, 1996). Dans un second temps, les comportements sociaux ont été observés en utilisant la taxonomie des comportements sociaux de Strayer (1980; voir également Trudel, Gauthier, Jacques et Strayer, 1983). Cette approche permet de faire ressortir l'impact social des gestes, puisque l'initiation des gestes et les réponses des pairs sont enregistrées. Les résultats de cette étude mettent en lumière, après une analyse des regroupements hiérarchiques ${ }^{4}$, trois styles d'adaptation: $l^{\prime}$ enfant sera amical, retiré ou engagé (Jeunier, Trudel et Legendre, 1997). Dans l'ensemble,

3. La collecte des échantillons de salive se fait à l'aide de boules de coton parfumées de poudre de fruits non sucrée que l'on demande aux enfants de mâcher. Une fois mâchées, les boules de coton sont insérées dans des seringues à usage unique, afin d'en extraire la salive. Les prélèvements de salive sont immédiatement congelés à $-20^{\circ} \mathrm{C}$. Ils sont ensuite analysés dans un laboratoire de biochimie pour estimer la concentration de cortisol. Les évaluations biochimiques sont réalisées par la méthode radio-immunologique.

4. L'analyse des regroupements hiérarchiques (cluster analysis) est une approche multidimensionnelle de traitement de données destinée à dégager des types d'individus en fonction de la similitude des profils individuels. Cette analyse typologique porte moins sur la relation entre les variables (approche R) que sur les variations intra-individuelles (approche Q) en tenant compte de l'ensemble des variables du construit sous étude; dans ce cas-ci, les individus associés à chacun des trois regroupements partagent une certaine similitude dans le répertoire comportemental. Nous suggérons la lecture du livre de Stephenson (1953) qui a introduit l'approche Q et a suggéré de multiples applications de cette méthode en sciences humaines. 
l'analyse des différents taux de cortisol en fonction des styles sociaux ne fait ressortir aucune différence significative. Cependant, les auteurs constatent une plus grande stabilité entre les deux mesures de cortisol chez les modes engagé et amical que chez le mode retiré. Ces résultats sont interprétés en fonction de la prédominance des modes d'expression affiliative et de la participation sociale chez les enfants amicaux et engagés. Les comportements des partenaires de jeu représentaient un élément important à considérer dans l'analyse de la régulation physiologique. En conclusion, les résultats de cette recherche illustrent les contraintes différentielles du contexte social immédiat, du fonctionnement à la fois physiologique et comportemental du jeune enfant, et appuient la perspective selon laquelle l'implication sociale active facilite la régulation qui soustend les processus biologiques. La démarche analytique privilégiée dans cette dernière étude ne tient cependant pas compte des différences individuelles quant aux taux de base du cortisol. C'est d'ailleurs le problème de plusieurs autres études sur le système endocrinien. En somme, les chercheurs s'interrogent sur ce qui lie les différences individuelles du taux de base du cortisol aux situations courantes de la vie de l'enfant.

Les travaux de Gunnar, Tout, de Haan, Pierce et Stansbury (1997) réalisés auprès des enfants de trois à cinq ans apportent un complément $\mathrm{d}$ 'information sur cette régulation biocomportementale. Les chercheurs ont en effet réussi à documenter le degré de réactivité du système HPS au cours des premiers jours d'intégration à un nouveau groupe de la garderie (contexte de pairs non familiers) et après quelques semaines de fréquentation de la garderie, soit au moment où les enfants se sont familiarisés les uns avec les autres (contexte de pairs familiers). Les résultats indiquent que les enfants qui manifestent un haut degré de réactivité du cortisol à la période initiale et une activation faible ou moyenne à la période ultérieure sont évalués comme compétents et plus populaires auprès de leurs pairs. En contrepartie, chez les enfants où l'on mesure des changements de réactivité passant de bas ou moyen à des niveaux élevés de cortisol et chez ceux qui maintiennent un degré élevé d'activité endocrinienne aux deux périodes, on observe une expressivité émotionnelle plus négative et une tendance à l'isolement social. Les chercheurs interprètent ces résultats comme le reflet de l'influence des différences individuelles et contextuelles. Avant cette étude, Gunnar, Resnick, Snidman (1987) avaient déjà démontré que l'inhibition comportementale pouvait être considérée comme une stratégie adaptative susceptible de contribuer à réduire le niveau d'engagement de l'enfant face à des situations imprévisibles. Ainsi, dans une nouvelle situation comme l'insertion dans un groupe en formation, on observe que le niveau de 
réactivité initiale de l'enfant timide sera plutôt faible. Par la suite, l'activation de l'axe HPS est plus probante lorsque l'enfant devient plus familier au groupe. Pour mieux rendre compte de ce schéma de régulation du cortisol, il y a donc lieu de reviser nos modèles actuels sur les construits du tempérament et du stress pour mieux tenir compte des contraintes contextuelles comme le niveau de familiarisation au groupe.

Granger, Stansbury et Henker (1994) ont pour leur part testé le protocole de la nouveauté sociale auprès d'un échantillon d'enfants de trois à six ans qui manifestent des problèmes de comportement. Leur étude s'échelonnait sur plusieurs sessions consécutives d'interaction en petits groupes de pairs. Dans l'ensemble, les résultats de cette dernière étude rejoignent les observations de Montagner, Henry, Lombardot, Benedini, Burnod et Nicolas (1978) en démontrant que les enfants d'âge préscolaire qui font preuve de leadership dans une situation de nouveauté sociale avec des pairs non familiers présentent les plus hauts taux de cortisol. Ils sont également engagés dans des interactions plus appropriées (moins agressives), ils sont moins isolés, moins tristes, moins craintifs et moins anxieux que les enfants ayant un niveau de cortisol stable ou faible.

Les résultats publiés par Gunnar et al. (1997), de même que ceux de Granger, Stansbury et Henker (1994), que nous venons de présenter, ne confirment cependant pas ceux rapportés par Lundberg, DeChateau, Windberg et Frankenhaeuser (1981) puisque ces derniers n'ont constaté aucune variation du taux de cortisol au cours des trois premières semaines d'école, parmi les enfants d'âge préscolaire fréquentant pour la première fois une garderie.

Nonobstant ces quelques divergences dans les résultats, que l'on peut attribuer à l'utilisation de protocoles différents, le relevé d'effets de plusieurs chercheurs semble suggérer que l'influence réciproque du comportement et de l'activité du système HPS en situation de nouveauté sociale pourrait jouer un rôle important dans le développement de problèmes de comportement à l'âge préscolaire. Ces résultats confirmeraient également l'interprétation de Gunnar (1994) selon laquelle on doit tenir compte des caractéristiques individuelles et contextuelles pour expliquer les différences que l'on observe dans la régulation du système hormonal.

Les travaux ayant évalué le lien entre le comportement social et les variations endocriniennes ont été réalisés principalement auprès d'échantillons d'enfants d'âge préscolaire. Ce n'est que très récemment que Davis, Donzella, Krueger et Gunnar (1999) ont entrepris l'étude des relations entre le cortisol et le tempérament au cours de la première semaine d'insertion des enfants à l'école primaire. Les résultats sont comparés aux 
taux de cortisol relevés au cours de la fin de semaine au domicile familial. Contrairement à ce qui se passe chez les enfants d'âge préscolaire, cette étude montre que ce sont moins les aspects intériorisés du tempérament (timidité et crainte) qui sont associés avec une augmentation de cortisol en situation de nouveauté, que les composantes extériorisées. On se trouve ici devant le problème de ne pouvoir distinguer les effets du stress de ceux de la nouveauté sociale. Un répertoire plus sophistiqué des comportements à l'âge scolaire pourrait expliquer l'augmentation du taux de cortisol chez les enfants plus actifs ou plus engagés sur le plan social.

En conclusion, la réactivité émotionnelle liée au stress et l'activation associée à la nouveauté sociale représenteraient deux conditions susceptibles de déclencher une réponse du système endocrinien. De là, notre difficulté à bien circonscrire la nature des stimuli et des contextes sociaux qui exigent une régulation biologique et comportementale. Pour Gunnar, Marvinney, Isensee et Fisch (1989), on ne peut prétendre que ce lien soit direct, mais l'augmentation des concentrations de glucocorticoïde influence inévitablement l'activité du système nerveux central, lequel en retour pourrait moduler les réponses comportementales. Chez l'enfant, l'activité comportementale peut elle-même contribuer à régulariser le fonctionnement du système adrénocortical. Toutefois, on ne peut être certain que les différences d'amplitude observées sur le plan comportemental sont nécessairement associées aux variations de l'activité adrénocorticale. Compte tenu de la disponibilité récente de techniques non intrusives pour évaluer le système HPS et, conséquemment, du peu de données empiriques, nos connaissances sur les contextes qui déclenchent une régulation physiologique sont plutôt limitées (Gunnar, 1989). Pour aller dans le sens de Tennes et Mason (1982), qui constataient que l'augmentation du taux de cortisol en réponse à un stress peut ne pas exister si l'enfant a déjà expérimenté la situation, nous ajoutons qu'il est essentiel de tenir compte de l'expérience sociale des enfants dans notre interprétation des réactions au stress et à la nouveauté sociale.

\section{L'ADAPTATION AUPRÈS DES PAIRS EN GROUPE STABLE}

Le bilan tiré des études sur le contexte de la nouveauté sociale nous amène à considérer la question de la régulation biocomportementale chez les groupes stables. Parmi les premières recherches réalisées dans ce domaine, il importe de souligner les travaux de Montagner qui ont été effectués en France vers la fin des années 1960. Ces recherches sont reconnues comme un des premiers efforts pour établir les liens entre des indices neuroendocrinologiques et le comportement social du jeune enfant (voir Montagner, 
Restoin et Henry, 1982). Ces études ont surtout porté sur la relation entre le rythme circardien du cortisol urinaire et la dominance sociale chez les enfants d'âge préscolaire. Ainsi, Montagner et al. (1978) rapportent que le statut de dominance serait corrélé avec les différents cycles circadiens du cortisol. Ils constatent également que les enfants dominants agressifs de deux et trois ans sécrètent plus de cortisol que les enfants moins agressifs; ceux-ci utilisant davantage des stratégies prosociales.

Une étude très récente de Dettling, Gunnar et Donzella (1999) effectuée auprès des enfants de trois à cinq ans de la garderie démontre qu'au milieu de la matinée il y a une légère augmentation du taux de cortisol. En outre, on observe que les plus jeunes enfants du groupe sont plus susceptibles de produire une activation du système HPS au cours de l'après-midi. L'activité neuroendocrinienne de ces enfants, lorsqu'ils sont évalués à leur domicile familial, suit cependant les variations habituelles du rythme circadien. Ces résultats suggèrent que les plus jeunes enfants et ceux faisant preuve d'immaturité sociale peuvent présenter des élévations de cortisol au fur et à mesure que la journée progresse.

À l'aide d'un protocole de recherche totalement différent, Goldstein, Field et Healy (1989) ont examiné dans quelle mesure la régulation neuroendocrinienne était affectée par le type de relation que l'enfant établit avec ses partenaires de jeu à la garderie. Les enfants sont regroupés en paires suivant les résultats d'une évaluation sociométrique ${ }^{5}$. Ils sont ensuite invités à participer à deux activités dans une salle de jeu d'un laboratoire, selon que les partenaires soient ou non des amis. On observe chez les dyades amies une plus grande concordance des comportements ludiques ainsi que des mesures du cortisol et du rythme cardiaque. Les auteurs interprètent cela en suggérant que la présence d'un ami atténue l'activité physiologique liée au stress en contexte de nouveauté sociale. Cependant, on ne peut établir clairement le lien de causalité de cette relation entre l'amitié et la régulation physiologique. Ainsi, le choix d'un ami est-il déterminé par certaines formes de compatibilité physiologique ou est-ce la nature des interactions entre pairs amis qui jouerait un rôle de régulation du système biologique? En dépit des contraintes de l'approche sociométrique, il importe de souligner la présence de liens complexes s'établissant entre les régulations sociale et physiologique chez les jeunes enfants.

5. La procédure sociométrique permet de faire ressortir les préférences des enfants dans la classe. Chaque enfant doit notamment identifier ses trois partenaires préférés. Le réseau social du groupe est dégagé de l'analyse de l'ensemble des choix de la classe. Ainsi, il est possible d'obtenir des scores de popularité pour chaque enfant du groupe. 
Mason concluait en 1959 que les stimulus naturels influençant le plus l'axe hypothalamo-hypophyso-surrénalien étaient les situations impliquant la nouveauté, l'incertitude et le non-contrôle (voir également Levine, 1983). Pourtant, dans l'ensemble les résultats de plusieurs travaux de recherche sont contradictoires. Pour expliquer cette polémique sur l'interprétation des données empiriques, Gunnar et al. (1989) proposent d'envisager la contribution de la nouveauté, de l'incertitude et de l'inconnu en fonction de la capacité des enfants à faire face aux événements. Ainsi, l'aspect stressant d'un événement ne serait pas précisément sa nouveauté ou son degré d'incertitude, mais plutôt l'incapacité pour l'individu de le contrôler ou de l'influencer et de gérer ses réactions émotives et comportementales en situation de stress.

Beaucoup d'études vont plus loin en suggérant que ce n'est pas l'absence des moyen de contrôle mais plutôt l'absence du sentiment de posséder les moyens de contrôle de la situation qui joue un rôle dans la production de cortisol. Stansbury et Gunnar (1994) ont observé que les enfants qui n'ont pas confiance dans leurs capacités de contrôler des événements menaçants produisent significativement plus de cortisol que les sujets qui sont confiants dans leur capacité de contrôler la situation.

\section{L'ADAPTATION BIOCOMPORTEMENTALE CHEZ LES ENFANTS ATYPIQUES}

Si nos connaissances sur les mécanismes de régulation biocomportementale ne sont pas à l'heure actuelle bien précises, on peut penser que l'étude des enfants atypiques pourrait apporter un complément d'information ou un éclairage différent sur certaines formes de dysfonctionnements physiologiques ou de comportements inadaptés. Observe-t-on chez les enfants qui ont des troubles du comportement des problèmes ou des modes particuliers de régulation physiologique en situation d'adversité?

Les travaux de recherche dans ce domaine furent fortement influencés par la conception voulant qu'il existe des marqueurs biologiques de l'agressivité ou de la violence. Plusieurs chercheurs ont ainsi noté chez les adultes atypiques (patients de cliniques psychiatriques ou personnes en milieu carcéral) une diminution du taux de cortisol associée à la présence de comportements d'agressivité (Virkunnen, 1985; Woodman, Hinton et $\mathrm{O}^{\prime} \mathrm{Neill}, 1978$ ). Retrouve-t-on les mêmes signes biologiques chez les enfants? 
Tennes et Kreye (1985) rapportaient qu'il existe chez les enfants d'âge scolaire une association négative entre le taux de cortisol urinaire et la fréquence des comportements agressifs envers les pairs (Tennes, Kreye, Avitable et Wells, 1986) ou hostiles envers les professeurs (Tennes et Kreye, 1985). Ils ont également noté une relation positive entre le degré de concentration du cortisol et la fréquence de comportements affiliatifs (Tennes et al., 1986). Vanyukov, Moss, Plail, Blackson, Mezzich et Tarter (1993) ont pour leur part effectué une étude auprès d'un échantillon de garçons dont les pères témoignent de troubles de personnalité antisociale et ont une histoire de troubles de conduite. Les résultats de cette recherche confirment que, chez ces garçons, moins le système HPS est activé, plus le nombre de symptômes de troubles de conduite est élevé. Les travaux effectués par McBurnett et ses collaborateurs apportent également une information pertinente à ce sujet: ils démontrent que le taux de cortisol des garçons de neuf ans référés à une clinique externe par suite d'un diagnostic de trouble de conduite ne se distingue pas de celui d'un groupe témoin (McBurnett, Lahey, Frick, Risch, Loeber, Hart, Christ et Hanson, 1991). Cependant, chez les enfants où l'on retrouve une comorbidité des troubles de conduite et des troubles d'anxiété, on observe des taux plus élevés de cortisol que chez ceux qui n'ont que des troubles de la conduite (McBurnett et al., 1991). Une étude plus récente de McBurnett, Lahey, Rathouz et Loeber (2000) démontre dans un échantillon restreint de garçons ayant des troubles de conduite, qu'un faible taux de cortisol salivaire est associé à des comportements agressifs. Les auteurs concluent que les concentrations d'hormones affecteraient la façon dont l'enfant réagit au stress et perçoit les menaces provenant de l'environnement. Seule note discordante dans ce bilan sur la relation entre le fonctionnement des systèmes neuroendocrinien et comportemental, Scerbo et Kolko (1994) ne rapportent aucune association significative entre le taux de cortisol salivaire et l'agressivité chez les enfants qui ont des troubles de conduite.

L'étude des bases endocriniennes associées aux troubles d'hyperactivité avec déficit d'attention a également fait l'objet de quelques études. Quoique peu de travaux aient été effectués auprès de ces enfants, les résultats se révèlent dans l'ensemble non concluants. Ainsi, Kruesi, Schmidt, Donnelly, Hibbs et Hamburger (1989) ne trouvent aucune différence dans l'activation du cortisol urinaire entre un groupe d'enfants hyperactifs avec déficit d'attention et un autre groupe d'enfants manifestant des troubles de conduite. Ces auteurs n'ont cependant pas vérifié les effets de la comorbidité. Schulz, Halperin, Newcorn, Sharma et Gabriel (1997) ont quant à eux comparé des groupes d'enfants agressifs et non agressifs qui ont été 
diagnostiqués hyperactifs avec déficit d'attention à l'aide du DSM-III-R. Les auteurs ne relèvent aucune différence de moyenne entre les deux groupes, ce qui infirme la présence de substrats endocriniens à l'hyperactivité chez les enfants durant la préadolescence.

En général, les études effectuées auprès des enfants atypiques se sont peu attardées sur les processus développementaux associés aux problèmes $\mathrm{d}^{\prime}$ inadaptation. C'est cependant le cas du suivi longitudinal réalisé par Granger, Weisz et Kauneckis (1994) qui a contribué à documenter les effets de la nouveauté sociale sur un groupe d'enfants qui ont des problèmes de comportement. L'étude constate qu'il y a, chez les individus ayant des problèmes intériorisés, une plus grande augmentation de sécrétion de cortisol entre la première session (pairs non familiers) et la deuxième session (pairs familiers). À l'opposé, chez les enfants qui ont des problèmes extériorisés (enfants agressifs et turbulents), on observe plutôt une dimininution de l'activation endocrinienne au fur et à mesure qu'ils se familiarisent avec leurs pairs. Ces résultats jettent un éclairage particulier sur les conclusions des autres chercheurs puisque les trajectoires développementales liant le cortisol et le comportement sont ici influencées par les caractéristiques de départ des enfants.

\section{BILAN CRITIQUE ET PERSPECTIVES DE RECHERCHE}

On doit tout d'abord retenir que la recherche sur la régulation biocomportementale chez les jeunes enfants est une jeune science; conséquemment, elle apparaît plus vulnérable à la critique tant sur le plan conceptuel que sur le plan méthodologique. On ne peut que constater le manque de synthèse qui serait susceptible de nous aider à mieux comprendre les processus organiques et comportementaux qui déterminent l'adaptation individuelle. De plus, une compréhension nuancée des mécanismes en jeu nécessiterait une approche plus interdisciplinaire intégrant, entre autres, les connaissances des sciences biomédicales et des sciences humaines.

Nonobstant ces considérations, il est pertinent de soulever un certain nombre de problèmes que rencontre cette nouvelle science. Ainsi, au plan méthodologique, on observe, à l'analyse de la recension de travaux, la présence de plusieurs incohérences au niveau des protocoles de recherche. Nous estimons que la divergence entre les résultats des travaux pourrait être attribuable à la diversité des devis de recherche utilisés. Les contraintes dans l'horaire des prises de données pourraient influencer considérablement les variations que l'on peut observer dans le taux de cortisol et, dans une certaine mesure, dans l'utilisation du répertoire 
comportemental des enfants. Dans le premier cas, on ne contrôle pas l'effet potentiel du rythme circadien. Plusieurs travaux n'indiquent même pas l'heure des prises de données! Dans le second cas, on pourrait se référer, par exemple, aux observations éthologiques de McGrew (1972) démontrant une évolution hebdomadaire du comportement des jeunes enfants en groupe de pairs. Le délai entre la mesure du cortisol et la situation environnementale qui induit le stress est également très variable d'une étude à l'autre. Ce problème est difficilement contournable puisque l'action du système HPS est progressive et atteint un pic de réactivité autour de la trentième minute après l'exposition à la situation de stress. Le processus organique sous étude rend donc très difficile la mise en place d'un protocole qui tiendrait simultanément compte de la régulation du cortisol et du comportement.

Notre recension des travaux de recherche fait également ressortir un problème d'inconsistance quant à l'utilisation des taux de base du cortisol. Comment apprécier la variation des indices du cortisol en situation de stress si nous n'avons aucune possibilité de la comparer avec des mesures qui correspondent au fonctionnement habituel du système endocrinien. Dans des situations expérimentales, les chercheurs peuvent se référer à des mesures pré-test à partir desquelles ils peuvent contraster les variations post-test. Cependant, dans le cas des recherches en milieu naturel, il est beaucoup plus difficile de choisir les moments et les contextes appropriés pour évaluer le taux de base du cortisol. Ainsi, Legendre et Trudel (1996) ont tenté de documenter cela en comparant les résultats des enfants en situation de nouveauté sociale avec ceux provenant de prélèvements de salive effectués aux mêmes heures mais une semaine plus tard au domicile familial. La solution apparaît élégante, mais connaissons-nous, dans ce cas, l'influence du climat familial ou de la qualité des relations entre les membres de la famille sur la régulation neuroendocrinienne de l'enfant? D'ailleurs, nous relevons très peu d'études ayant abordé l'analyse de la relation entre le taux de cortisol et le fonctionnement comportemental de l'enfant dans le contexte familial. À cette contrainte s'ajoute celle de la valeur du taux de base, puisque certains enfants pourraient sécréter des concentrations élevées de cortisol même en situation qu'on estime non stressante. Dans l'ensemble, nous devons privilégier l'utilisation de protocoles de recherche qui tiennent compte des caractéristiques biocomportementales de l'individu et de l'influence potentielle des contextes habituels de vie de l'enfant. L'étude des contextes naturels qui induisent une adaptation biosociale doit se faire plus systématiquement si l'on veut dégager une compréhension nuancée de l'interaction entre environnement, comportement et régulation physiologique. 
Un autre aspect qui retient notre attention est la grande variabilité intraindividuelle des données sur le cortisol. Chez certains enfants on voit des taux très comparables entre les mesures répétées du cortisol en situation de non-stress, alors que chez d'autres, on retrouve des taux très instables. À ce propos, il n'est pas rare d'observer de grandes variations intraindividuelles et interindividuelles au niveau des mesures physiologiques. En outre, ces variations pourraient être interprétées comme un reflet du niveau de plasticité biologique du système endocrinien; cellesci correspondraient-elles également à une plus grande plasticité biocomportementale? Un dernier constat sur les contraintes méthodologiques porte sur l'importance de l'observation directe pour évaluer le profil comportemental de l'enfant. Quelques études utilisent le rapport d'un des parents ou de l'enseignante pour tracer le portrait comportemental de l'enfant. Comme le contexte social immédiat est une des composantes essentielles dont on doit tenir compte dans l'étude de la régulation biocomportementale, nous considérons qu'il n'est pas opportun de ne favoriser que l'utilisation de mesures indirectes pour établir le profil de l'enfant et la description du contexte social. Bref, sur le plan méthodologique, il est difficile d'apporter une conclusion définitive sur les processus $\mathrm{d}^{\prime}$ interaction biosociale si l'on n'est pas en mesure d'assurer une compatibilité minimale des protocoles d'évaluation entre les diverses études.

Au cours de la dernière décennie, on décèle un intérêt croissant pour intégrer des mesures biologiques aux protocoles des études sur le comportement. Devant l'accessibilité récente d'une technologie non intrusive pour mesurer la variabilité des taux de cortisol, il n'est pas surprenant d'observer un tel engouement pour l'adaptation biologique au stress. Toutefois, l'intégration théorique de cette nouvelle information se heurte constamment à la difficulté d'interpréter les résultats empiriques en fonction de paradigmes où la valeur adaptative du comportement $\mathrm{n}^{\prime}$ est pas toujours clairement démontrée. Ainsi, pour les tenants d'une approche génétique du comportement, on doit anticiper que les réponses physiologiques de l'organisme sont relativement stables dans le temps et que l'état endogène, ou physiologique, contribue fortement à régulariser le comportement. Le point de vue systémique aborde plutôt la question en examinant si ces processus hormonaux sont liés aux effets du contexte social sur le comportement de l'enfant, comment ces processus s'intègrent au comportement de l'enfant au cours du développement et si ces influences réciproques contribuent à l'adaptation psychosociale ultérieure ou à plus long terme (Magnusson, 1988). La perspective biosociale met donc plus l'accent sur la nature dynamique des relations entre la physiologie et le comportement. En ce sens, on conçoit que l'interaction de l'environne- 
ment et des variations du fonctionnement biologique impose constamment des contraintes à l'adaptation comportementale de l'individu. Dans un modèle systémique ou holistique du développement, on doit conceptualiser ces différentes composantes comme des systèmes intégrés agissant constamment sur l'individu et orientant sa trajectoire développementale. Il ne s'agit plus d'une perspective orientée vers l'analyse des rapports de causalité où la notion de déterminisme est accentuée, mais bien d'une approche probabiliste postulant la présence d'une certaine plasticité biocomportementale au cours du développement épigénétique. Autrement dit, si les gènes influencent les contextes de développement, ces contextes influencent également l'expression des gènes; les trajectoires de développement sont le reflet de ces effets réciproques ou bidirectionnels (Gottlieb, 1991). Dès lors, l'analyse des facteurs associés à chacune de ces composantes ne peut se faire sans tenir compte à la fois des variations entre les variables et de la variabilité intra-individuelles; dans ce dernier cas, nous devons examiner d'un point de vue multidimensionnel les sentiers de développement des enfants qui ont le même profil. Nous privilégions alors une approche plus centrée sur la personne pour faire ressortir des sousgroupes d'enfants dont le style d'adaptation est semblable (voir Magnusson, 1998). En résumé, il importe de considérer la diversité des processus par lesquels la biologie, l'environnement et le comportement interagissent continuellement et vont déterminer des trajectoires spécifiques de développement (Gottlieb, 1993; Gottlieb, Wahlsten et Lickliter, 1998; Lerner, 1991).

Une telle approche exige une description adéquate des variables contextuelles et organiques à différentes périodes de la vie et dans différentes situations d'adaptation (Cairns, 1991; Cairns et Cairns, 1994). L'étude des processus biocomportementaux nécessite également de mieux cerner les mécanismes de régulation biologique qui agissent en situation d'adversité. Dans un tel contexte, nous ne pouvons plus limiter l'étude de l'adaptation individuelle à des mesures de taux de cortisol (Levine et Coe, 1985); cette hormone n'est que le produit final d'une chaîne de réaction plus générale liée à l'activation de l'axe HPS. Enfin, le rôle que le comportement joue dans le système biosocial doit être clarifié. Le comportement $\mathrm{n}^{\prime}$ est pas uniquement le produit de l'interaction des contraintes organiques et environnementales, mais correspond aux ressources adaptatives dont dispose l'individu pour gérer l'adversité. Le répertoire individuel qui se construit au cours du développement contribue directement à la régulation du système biocomportemental. 


\section{FIGURE}

Le processus de régulation du système endocrinien en situation de stress

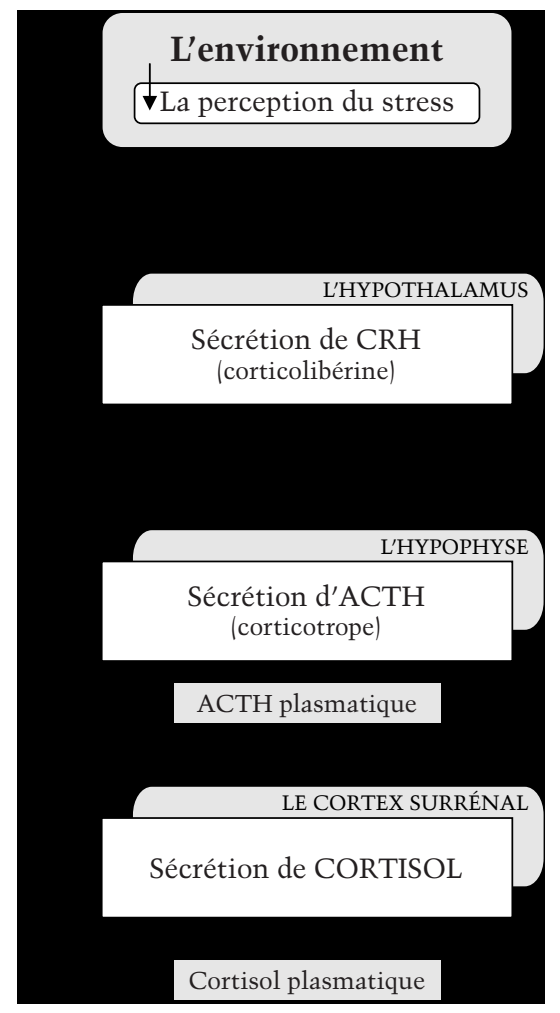




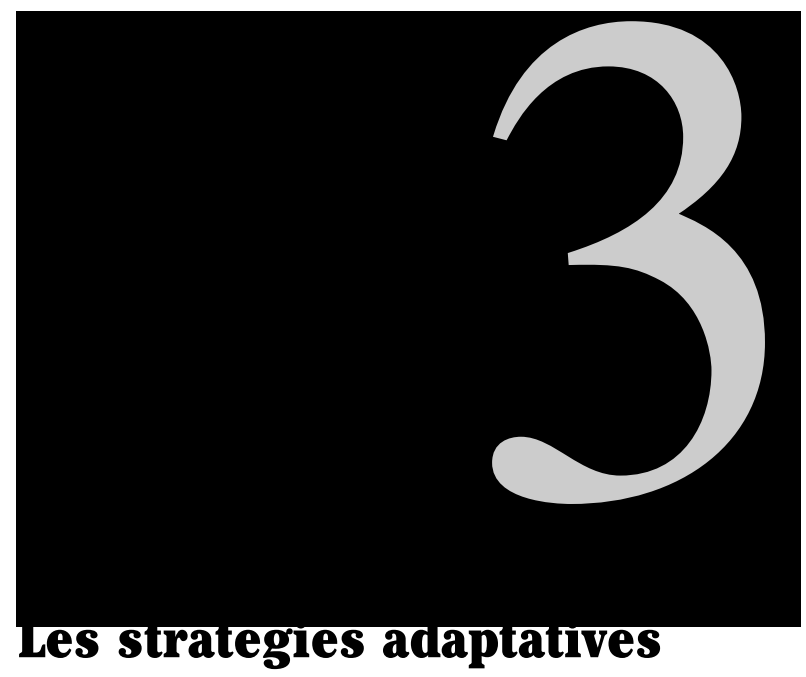

MiChELLE DUMONT

Département de psychologie

Université du Québec à Trois-Rivières

Trois-Rivières (Québec) 

E ncore aujourd'hui, on connaît très mal comment les enfants réagissent lorsqu'ils sont confrontés à des situations stressantes qui nécessitent une adaptation. Très souvent, et avec raison, on utilise les écrits traitant de cette problématique à partir de modèles adultes pour expliquer les mécanismes adaptatifs, aussi appelés "coping", qu'utilisent les enfants lorsqu'ils sont confrontés à des difficultés. Cet emprunt aux théories adultes découle certes du manque évident de données empiriques pour décrire les mécanismes de coping tôt dans le développement. Pourtant, un des intérêts de l'étude des mécanismes adaptatifs durant l'enfance est de mieux comprendre leur rôle potentiel dans le développement de la personnalité et l'apparition des troubles psychologiques et physiques qui pourraient se manifester plus tard par suite d'une mauvaise adaptation à une ou plusieurs situations de stress. Un autre attrait de cette étude est de mieux comprendre la genèse du coping dans le but, cette fois, d'établir des ponts conceptuels avec d'autres théories du développement. Par exemple, le développement cognitif influence-t-il le mode de coping à différentes périodes du cycle de la vie? Les principaux agents de socialisation rencontrés au cours de la vie (les membres de la famille, les pairs, le conjoint, les collègues de travail) influencent-ils à des degrés divers le développement et le choix de la réponse de coping ? Quel est le poids différentiel des caractéristiques individuelles, familiales et environnementales (sociales, matérielles...) dans le développement des différents modes de coping? Grâce aux études longitudinales et transversales, la réponse à ces différentes questions permettra de préciser les cibles des interventions cliniques et, ce faisant, d'augmenter l'efficacité du coping adopté à différentes périodes du cycle de la vie.

Ce chapitre traite des stratégies adaptatives à partir de modèles théoriques adultes et des rares résultats de recherche obtenus auprès des enfants. Plus spécifiquement, nous traiterons trois dimensions importantes de ce champ d'études: d'abord, nous définirons le concept de coping à l'intérieur des principaux modèles théoriques et nous opposerons la notion de "style adaptatif " à celle de "stratégie adaptative». Ensuite, nous présenterons les styles de coping les plus souvent rapportés et 
soulignerons les conditions nécessaires à leur efficacité. Enfin, nous aborderons les changements développementaux dans l'utilisation du coping au cours de l'enfance et décrirons le lien entre le mode de coping et l'adaptation psychosociale.

\section{DÉFINITIONS ET MODÈLES THÉORIQUES}

D'un point de vue historique, la recherche sur le coping est devenue très populaire dans les années 1980, après les publications de Pearlin et Schooler (1978) et tout particulièrement depuis celle de Lazarus et Folkman (1984). Il se dégage de ces ouvrages majeurs deux théories du coping qui reflètent assez bien l'état actuel des travaux de recherche s'inspirant en particulier de l'approche contextuelle et de l'approche psychodynamique.

L'approche contextuelle, communément appelée théorie transactionnelle du stress " personne-environnement ", fut proposée en premier par Lazarus en 1966, puis développée en 1984 dans un ouvrage majeur écrit en collaboration avec Folkman. Cette relation "personneenvironnement " résulte de l'influence multidimensionnelle entre l'environnement social et physique, les buts personnels, les croyances à propos de soi et du monde et les ressources personnelles. Plus spécifiquement, Lazarus et Folkman définissent le coping comme l'ensemble des efforts cognitifs et comportementaux (ou encore des processus qu'un individu interpose entre lui et l'événement perçu comme menaçant) qui permettent de maîtriser, de tolérer, d'éviter ou de minimiser l'effet négatif du stress sur le bien-être physique et psychologique d'une personne. Les réponses de coping, conscientes et diversifiées, ont pour but, si elles sont efficaces, de favoriser l'adaptation psychosociale, de maintenir une image positive de soi, de réduire l'inconfort psychologique et de maintenir une relation satisfaisante avec son environnement. Il va de soi que l'usage du terme “ coping " implique l'existence d'un problème ou d'un stresseur et la mise en place d'une réponse spécifique pour y faire face. Ces " efforts " de coping varient selon les demandes ou les contraintes d'une situation, la signification qui lui est accordée (l'évaluation cognitive serait un facteur médiatisant le lien entre le stresseur et la réponse de coping; Berg, Meegan et Deviney, 1998) ainsi que les ressources personnelles, familiales, environnementales dont dispose un individu. D'un point de vue développemental, on peut se demander s'il n'existe pas des différences cognitives dans la signification accordée aux situations stressantes (comme éprouver un sentiment de menace plus grand avec l'âge étant donné que la personne risque d'avoir une meilleure compréhension du 
potentiel de dangerosité associé à une situation donnée), ou encore s'il n'existe pas des préférences individuelles quant à la mobilisation des ressources personnelles, familiales ou environnementales selon l'âge (comme s'appuyer davantage sur ses ressources personnelles avec l'âge) et, enfin, si les différences développementales observées dans la réponse de coping ne seraient pas imputables à la spécificité des sources de stress liées à l'obligation de réaliser ses tâches développementales (comme le stress financier plus présent à l'âge adulte ou le stress scolaire plus fréquent durant l'enfance et l'adolescence).

Un des éléments clés de l'approche contextuelle de Lazarus est certes de concevoir le coping comme un processus dynamique, c'est-àdire comme pouvant changer non seulement dans le temps mais aussi en fonction des demandes endogènes (comme les changements pubertaires) et exogènes (comme le divorce des parents), sans oublier le rôle fondamental de l'évaluation cognitive (faut-il définir le problème comme l'expression d'un sentiment de perte, de menace d'une perte ou d'un défi ?). En d'autres mots, face à une situation potentiellement stressante, la personne détermine s'il y a un risque pour son bien-être psychique et physique. Si la personne se sent en danger, elle devra alors examiner les ressources de coping dont elle dispose pour y faire face. Cette approche prévoit donc une variabilité intra-individuelle de la réponse de coping, puisque celle-ci dépendrait des circonstances, de l'évaluation cognitive et des ressources dont la personne dispose à différentes périodes du cycle de la vie. En quelque sorte, l'approche contextuelle propose que ce sont les situations qui forcent la personne à choisir ses réponses de coping et non l'inverse (Holahan, Moos, et Schaefer, 1996).

L'approche psychodynamique, à l'opposée de l'approche contextuelle, tire son origine conceptuelle de la théorie des mécanismes de défense développée par Freud (1933; cité dans Zeidner et Saklofske, 1996). Cette approche vise à mieux décrire les processus inconscients dans la réduction des tensions et la gestion des conflits (menaces internes). Selon Paulhan et Bourgeois (1995), le coping est apparenté aux défenses du Moi (déni, intellectualisation) dont la finalité est de diminuer ou de supprimer tout ce qui peut susciter l'angoisse. À partir des travaux classiques de Haan (1977) et de Vaillant (1977), pour ne nommer que ceux-là, les mécanismes défensifs ont été catégorisés et hiérarchisés en stratégies plus ou moins adaptatives, plus ou moins matures (défenses immatures: projection, hypocondrie; défenses matures: humour, anticipation). Par opposition à la production d'une réponse consciente, volontaire et adaptée à une situation (point de vue selon Lazarus), l'approche psychodynamique suggère des préférences individuelles relativement stables pour certains 
mécanismes défensifs ou styles de coping permettant de faire face aux conflits (Vaillant, 1977). Cette position suggère l'existence possible d'un pattern intraindividuel et relativement stable tel un trait de personnalité. Cette conception du coping s'oppose au processus dynamique proposé par Lazarus et ses collaborateurs, et dans lequel la réponse de coping varie selon les circonstances, les réflexions, la disponibilité des ressources et l'espace-temps.

Si le modèle psychodynamique contribue à une meilleure compréhension des mécanismes de coping, selon Paulhan et Bourgeois (1995), il explique plus la réduction des tensions (régulation des émotions) que la résolution d'un problème créé par l'événement stressant. Or ces deux fonctions du coping sont très présentes dans la théorie de Lazarus, comme en témoigne sa classification bidimensionnelle, à savoir le coping centré sur le problème et le coping centré sur les émotions. Or, à partir de divers résultats de recherche, Paulhan et Bourgeois constatent qu'une définition complète du coping nécessite d'inclure simultanément les fonctions de régulation émotionnelle et de résolution de problème afin de permettre à l'organisme de mieux s'ajuster à un événement qu'il perçoit comme menaçant ou dangereux. En effet, la réponse comportementale se présente souvent en même temps que la réponse émotionnelle, bien que la séquence comportement-cognition-émotion ou à l'inverse émotioncognition-comportement, ne soit pas encore clairement documentée. Ces auteurs font aussi remarquer que les mécanismes d'adaptation psychiques inconscients, dont la fonction première est de maintenir l'anxiété dans des limites supportables, diffèrent des habiletés de coping qui sont des techniques apprises pour permettre à l'individu d'affronter consciemment son anxiété, alors que le déploiement des mécanismes de défense n'est pas volontaire mais bien inconscient.

En somme, ces deux approches théoriques suggèrent l'existence de stratégies adaptatives (selon Lazarus et Folkman) et de traits ou styles adaptatifs (selon Haan et Vaillant) afin de faire face aux pressions internes et externes (changements mineurs ou majeurs, chroniques ou ponctuels) qui surviennent au cours du développement. Plus concrètement, adoptet-on le même type de coping dans un contexte d'examen, de perte relationnelle? Les tenants de l'approche contextuelle de Lazarus croient que l'individu utilise un répertoire d'options de coping (pensées, comportements, émotions) en fonction de l'efficacité qu'il croit avoir face à la situation qui se présente, de ses ressources personnelles, familiales et environnementales et de la signification qu'il accorde à la situation stressante (menace, perte ou défi). Chez ceux qui défendent l'approche psychodynamique, on pense plutôt que l'individu utilise une manière 
constante de s'adapter, peu importe la nature de la situation stressante. Or, force est de constater que cette approche, en proposant un trait de personnalité comme style de coping, permet beaucoup moins d'identifier les changements qui s'opèrent dans le processus de coping à différents stades d'une crise (on n'adopte pas toujours la même réponse de coping au début, au milieu ou à la fin d'une période de crise en voie de résolution). Par conséquent, il faut bien reconnaître que les traits de personnalité sont à eux seuls insuffisants pour prédire, dans la plupart des situations stressantes, la manière dont un individu fera face au stress et le degré d'efficacité qu'il en percevra. Malgré 1'opposition entre ces deux points de vue théoriques, il serait beaucoup plus utile d'adopter une approche intégrative, comme le proposent Schwarzer et Schwarzer (1996). En effet, une personne peut ne pas adopter exactement la même stratégie dans toutes les circonstances stressantes, mais peut aussi avoir tendance à sélectionner un comportement spécifique qui appartient à la même classe de stratégies, comme les stratégies regroupées sous le style " évitant » ou " affrontant ». Autrement dit, les individus peuvent généraliser dans les situations un nombre limité de stratégies qu'ils réutilisent à différentes occasions. Par ailleurs, si ces deux approches théoriques diffèrent quant à la manière de concevoir la réponse de coping (réponse consciente versus inconsciente; stratégie adaptative versus trait ou style adaptatif), tous les chercheurs s'accordent pour dire que le coping vise à maintenir l'adaptation psychosociale d'une personne pendant les périodes de stress.

\section{STYLES DE COPING ET DEGRÉ D’EFFICACITÉ}

Plusieurs concepts ont été proposés pour analyser le coping, à savoir les ressources de coping, les styles de coping et les efforts de coping. Pour la clarté du propos, Sandler, Wolchik, MacKinnon, Ayers et Roosa (1997) établissent une distinction conceptuelle entre ces trois notions. Les ressources de coping sont des caractéristiques individuelles relativement stables qui influencent la manière dont l'individu s'adapte dans les situations stressantes (tempérament facile, estime de soi, optimisme, contrôle interne ou externe, habilité de résolution de problèmes). Les styles de coping sont les préférences habituelles dans la manière d'aborder les problèmes face à un éventail de stresseurs (style “ approche " ou “évitement»). Les efforts de coping représentent des stratégies comportementales et cognitives produites dans des situations stressantes spécifiques et pouvant changer selon la nature des pressions exercées sur l'organisme. Dans cette deuxième section, nous présenterons les styles de coping les 
plus souvent observés au cours du développement. On peut distribuer ces principaux modes d'adaptation en deux groupes, à savoir le coping centré sur le problème, ou encore centré sur les émotions (Lazarus et Folkman, 1984) et le coping de type "approche " ou "évitement " (Ebata et Moos, 1994; Roth et Cohen, 1986).

Le coping centré sur le problème vise à contrôler ou à modifier la source de stress, comme un coping d'affrontement, et à remédier à une situation menaçante ou nuisible par des efforts de résolution et de reconceptualisation (faire des plans, chercher des informations, développer de nouvelles habiletés, générer des solutions de rechange). Le coping centré sur les émotions cherche quant à lui à ventiler, à gérer ou à pallier une situation par une réponse émotionnelle adaptative ou non adaptative (stratégies d'auto-préoccupation, fantaisies, régulation consciente de l'affect, recherche de soutien émotionnel; lire Parker et Endler, 1996). Autrement dit, lors d'une situation stressante, on peut affronter le problème en faisant des efforts actifs pour le solutionner ou encore essayer d'éviter le problème en mettant son attention sur la gestion des émotions (Holahan et al., 1996). Dans le dernier cas (coping centré sur les émotions), il s'agit de stratégies cognitives qui ne changent pas directement la situation mais qui aident à développer une autre façon d'évaluer la situation. Elles ne sont donc pas " passives " et peuvent nécessiter une restructuration interne qui demande beaucoup d'efforts (Schwarzer et Schwarzer, 1996).

Dans un article de référence, Roth et Cohen (1986) présentent le coping de type approche/évitement. Pour fins de clarté, Wills et Hirky (1996) ont établi une correspondance entre les types de coping centré sur le problème ou centré sur les émotions de la tradition de Lazarus et le coping approche/évitement. Par exemple, le coping centré sur le problème se rangerait sous la rubrique " approche " puisqu'il implique l'investissement actif d'efforts dans le processus de coping (résolution de problèmes, recherche d'informations et de soutien social). En revanche, le coping centré sur les émotions se rangerait plutôt sous la rubrique “évitement " qui suppose des efforts cognitifs, comportementaux ou émotionnels visant à s'éloigner du stresseur par le déni, la distraction, la rêverie, la ventilation des émotions négatives, l'acceptation ou la résignation, voire même la consommation de substances nocives pour la santé.

Comment peut-on établir l'efficacité du coping? Malgré les recherches abondantes dans le domaine, nous sommes loin de pouvoir définir le coping optimal pour un individu donné, dans une situation donnée, à un âge donné, dans une culture donnée. C'est sans doute parce que l'efficacité 
d'une stratégie dépend de la nature du stresseur, de la perception du groupe social et culturel auquel on appartient ainsi que de la maturité et des ressources bio-psycho-sociales d'un individu. Pour Schwarzer et Schwarzer (1996), l'efficacité du coping dépend aussi de l'étape dans laquelle l'individu se situe lors d'une crise. En effet, un coping peut être adaptatif dans la première phase et inefficace dans la seconde, alors qu'une approche complètement différente pourrait être adoptée dans la dernière phase. Ce faisant, la stabilité du mode de coping n'est peut-être pas souhaitable, puisque l'individu est censé s'adapter aux exigences spécifiques de la situation à laquelle il doit faire face. Zeidner et Saklofske (1996) partagent cet avis et croient qu'on ne devrait pas établir une distinction entre coping adaptatif ou mal adaptatif, mais plutôt suggérer que, dans certaines circonstances, un mode de coping favorise des conséquences adaptatives. Selon Kohn (1996), tant le coping “ actif " (centré sur le problème) que le coping " passif » (centré sur les émotions ou l'évitement) constituent des modes efficaces d'adaptation, et cela dans des circonstances différentes, notamment si on tient compte du degré de contrôle que pense avoir la personne dans une situation donnée. Cette distinction constitue certes un pas important dans notre compréhension du coping. En effet, si l'efficacité du coping varie selon les circonstances, la plupart des gens optent pour un coping actif dans des situations perçues comme contrôlables qui suscitent un faible niveau de détresse émotionnelle (Compas, Malcarne, et Fondacaro, 1988). Plus spécifiques, les gens optent généralement pour un coping centré sur le problème dans les situations où ils pensent que quelque chose de constructif peut être fait ou qu'ils ont besoin de plus d'information (Ebata et Moos, 1991; Folkman et Lazarus, 1980). Roth et Cohen (1986), et plus récemment Terry (1994), concluent que les stratégies d'approche sont plus efficaces dans une situation où il $\mathrm{y}$ a un potentiel de contrôle, tandis que les stratégies d'évitement sont plus adaptées dans une situation perçue comme incontrôlable. Cependant, si le coping centré sur les émotions (dédramatiser, espérer un miracle) ou celui visant l'évitement (nier, se distraire) aident à maintenir un équilibre psychologique et à réduire l'anxiété, Zeidner et Saklofske (1996) pensent qu'une réponse adaptative pouvant remédier à une situation menaçante requiert généralement un coping centré sur le problème.

Malgré l'efficacité du coping dit "actif " dans la réduction du stress, car il procure un sentiment de maîtrise, le coping d'évitement (déni, pensée magique, distraction mentale) peut aussi être tout à fait adaptatif s'il offre un moment de répit pour respirer psychologiquement et fuir la pression liée à une situation stressante, surtout si la personne n'est pas 
encore prête à l'affronter (Carver, Scheier, et Pozo, 1992). Ce n'est qu'après un certain temps, une fois qu'elle est devenue plus forte, que la personne peut faire face à la réalité et aborder le problème d'une manière plus pratique et plus centrée sur le problème.

Un coping est dit adaptatif s'il mène à la résolution permanente du problème et s'il maintient un état émotionnel positif, c'est-à-dire s'il permet au sujet de maîtriser ou de diminuer l'impact de l'agression sur son équilibre physique et psychologique. L'efficacité du coping réside donc dans l'habileté à réduire la détresse immédiate aussi bien qu'à renforcer le bien-être dans le futur. En s'inspirant du travail de Meneghan (1982) et de Pearlin et Schooler (1978), Zeidner et Saklofske (1996) proposent un sous-ensemble de critères permettant d'évaluer l'efficacité du coping: 1) résolution du conflit ou de la situation stressante; 2) réduction des réactions biochimiques et physiologiques (réduire la pression sanguine, le rythme cardiaque, la transpiration; 3) réduction de la détresse psychologique en conservant l'anxiété dans des limites acceptables; 4) fonctionnement social normal; 5) retour aux activités routinières précédant l'agent stresseur; 6) bien-être pour soi et pour les personnes affectées par la situation ; 7) maintien d'une estime de soi positive; 8) efficacité perçue dans l'utilisation de la stratégie jugée aidante.

En intervention clinique, aider une personne à adopter un bon coping, c'est lui donner le pouvoir de contrôler sa vie et d'influencer son développement. Autrement dit, un bon coping devrait aider une personne à mieux sélectionner les contextes de défis et à éviter les situations accablantes, à anticiper les problèmes, à évaluer les situations avec optimisme et réalisme, et à réagir de façon proactive, intentionnelle, organisée, flexible et bien coordonnée selon ses ressources personnelles et sociales (Skinner et Edge, 1998). Lepore et Evans (1996) suggèrent que l'efficacité du coping dépend non seulement des ressources de coping, c'est-à-dire des caractéristiques personnelles (l'optimisme, le sentiment d'efficacité, l'estime de soi positive, les ressources biologiques, matérielles et environnementales), mais également des réponses spécifiques de coping pour faire face adéquatement au stresseur (mode approche/évitement, comportement, cognition). La flexibilité qu'a la personne de s'adapter aux exigences d'une situation ainsi que l'étendue de son inventaire de coping constituent des atouts indéniables en faveur de son adaptation, comparativement à une personne qui aurait un répertoire plus limité ou plus rigide. Soulignons aussi que le coping adopté face à un stresseur peut augmenter ou diminuer l'habileté ou la motivation d'une personne à faire face à un autre stresseur. En effet, Lepore et Evans (1996) soulignent le lien bidirectionnel entre l'efficacité du coping et le développement des 
ressources personnelles. Par exemple, un individu qui aurait le sentiment de maîtriser son environnement ou d'avoir une meilleure estime de soi par suite d'un coping efficace pourrait développer plus de confiance personnelle et du même coup se sentir moins menacé par la présence d'un autre stresseur. Enfin, rappelons que le coping est une réponse à des émotions, généralement négatives, produite en réaction à un événement. Certaines personnes éprouvent toujours les mêmes difficultés face aux situations difficiles et ne se relèvent jamais, alors que d'autres traversent efficacement les périodes plus obscures et ressortent grandies de ces expériences. On dit de ces dernières qu'elles sont “ résilientes ».

L'amélioration des mécanismes adaptatifs constitue une des voies les plus prometteuses pour prévenir le développement des problèmes biopsycho-sociaux durant l'enfance et, plus tard, à l'adolescence et à l'âge adulte. La question des influences réciproques entre coping $<->$ détresse a été soulevée par Zeidner et Saklofske (1996). Par exemple, est-ce que le lien entre un coping particulier et peu de symptômes de détresse signifie que le coping réduit la détresse (coping ---> santé/détresse) ou n'est-ce pas plutôt que la personne qui a peu de problèmes ou qui a une meilleure santé mentale et physique tend à utiliser des stratégies de coping très particulières (santé/détresse ----> coping) ? Pour illustrer le lien coping-détresse, nous vous présentons les résultats de différentes recherches qui illustrent la relation existant entre le coping et l'adaptation psychosociale durant l'enfance, et plus particulièrement les études évaluant le coping évitant, souvent associé au coping émotionnel, et le coping centré sur le problème.

Kliewer et Sandler (1993) rapportent, à partir d'un inventaire de coping d'enfants de 7 à 13 ans, une relation positive entre le coping évitant et les symptômes dépressifs. Plus spécifiquement, les résultats indiquent que les enfants considérés par leur enseignant comme les plus compétents socialement, mais ayant aussi des symptômes dépressifs, adoptent une façon plus défensive d'aborder les problèmes que les enfants qui sont compétents socialement mais qui n'ont aucun symptôme dépressif. Extérieurement, ces deux types d'enfants semblent fonctionner de façon identique (ils vont avec les autres pairs, démontrent de l'empathie et de la sensibilité aux autres, gèrent leurs affects) et ce n'est que lorsqu'on explore leur monde interne que l'on découvre d'importantes différences dans la manière de réagir à leur environnement. Cela suggère que les enfants qui semblent compétents peuvent vivre une réalité interne bien différente. Plus récemment, Lengua et Sandler (1996), à partir d'un inventaire de coping d'enfants de 8 et 12 ans, rapportent des résultats similaires et trouvent que le coping évitant corrèle positivement avec les symptômes psychologiques, alors qu'il n'y aurait pas de relation significative entre le 
coping actif et la détresse psychique. Devant ce type de résultats, il importe de réduire l'utilisation du coping évitant dans le répertoire des stratégies adaptatives d'un enfant, surtout lorsqu'il peut encore changer quelque chose à la situation stressante à laquelle il fait face.

\section{APPROCHE DÉVELOPPEMENTALE ET RELATION COPING-SANTÉ MENTALE}

Dans cette section, nous soulignerons les différences développementales de trois types de coping souvent adoptés durant l'enfance: le coping cognitif, le coping social et le coping émotionnel. Plus spécifiquement, l'inventaire des stratégies adaptatives est-il stable au cours du développement ou se transforme-t-il sous la pression de l'accomplissement de nouvelles tâches développementales? Quel aspect du développement socioémotionnel, moral et cognitif influence l'acquisition et le choix de la réponse de coping? Encore aujourd'hui, très peu d'études décrivent, d'un point de vue développemental, les différences interindividuelles dans la continuité ou la discontinuité du mode de coping, de ses mécanismes d'acquisition (Losoya, Eisenberg, et Fabes, 1998) et de ses fonctions différentielles suivant les changements liés au développement (Berg, Meegan, et Deviney, 1998). Même si le fait d'utiliser les modèles de coping adultes constitue un point de départ nécessaire à la compréhension des stratégies adaptatives adoptées durant l'enfance, le coping des enfants risque de différer de celui des adultes, non seulement à cause des différences de maturité socioémotionnelle et cognitive, ce qui, par ricochet, peut faire varier la perception du stress et la réponse de coping, mais aussi en raison de l'influence familiale plus directe durant l'enfance et de celle des pairs, du conjoint et des collègues de travail à mesure que l'individu vieillit.

\section{LE COPING COGNITIF}

À mesure que l'enfant se développe, il acquiert de nouvelles compétences intellectuelles qui peuvent l'aider à faire face aux difficultés de la vie. Malgré ces nouvelles acquisitions, l'enfant, avec l'âge, devient de plus en plus conscient des menaces qui l'entourent, anticipe des scénarios catastrophiques et devra prendre la responsabilité de ses réponses de coping. $\mathrm{Du}$ point de vue du développement intellectuel, on peut penser que l'émergence de la pensée abstraite à l'adolescence (la pensée hypothéticodéductive, ou pensée scientifique, et la capacité de planification) favorise l'utilisation de stratégies cognitives de plus en plus complexes pour 
résoudre des situations problématiques (Compas, 1998). En effet, certaines recherches rapportent une augmentation du coping cognitif avec l'âge, ce qui inclut parfois des stratégies comme l'analyse logique ou la réévaluation positive (Ebata et Moos, 1994; Seiffge-Krenke et Shulman, 1990).

Voici quelques résultats de recherche soulignant la place du coping cognitif durant l'enfance. Altshuler et Ruble (1989) ont évalué les réponses de coping d'enfants âgés de 5 à 12 ans lors d'une tâche de résolution en situation incontrôlable impliquant soit une frustration (attendre pour obtenir un objet désiré), soit une peur (attendre dans la salle du dentiste). Les résultats indiquent que les stratégies adaptatives de type distraction cognitive (manipulation de ses états mentaux en mettant son attention sur ses pensées) augmentent avec l'âge et que les stratégies de type distraction comportementale (entreprendre une activité physique comme jouer à un jeu ou lire un livre pour se divertir de son problème) sont proposées par tous les enfants peu importe l'âge. Plus récemment, Altshuler, Genevro, Ruble et Bornstein (1995) rapportent une relation positive entre l'âge et les stratégies de distraction cognitive auprès d'enfants âgés de 5 à 11 ans hospitalisés pour une opération. À partir d'entrevues individuelles, Hoffner (1993) évalue le mode de coping d'enfants âgés de 6 à 12 ans dans des situations incontrôlables qui nécessitent la régulation des émotions (une intervention chez le dentiste, une chez le docteur, de la turbulence dans un avion, l'attente du résultat d'un examen important qu'on croit avoir échoué). Globalement, les stratégies de type blunting (évitement, distraction ou réévaluation de la situation pour la rendre moins menaçante) sont plus souvent rapportées, surtout à mesure que l'on vieillit, viennent ensuite les stratégies de monitoring (poser des questions à propos de la menace) et enfin, la recherche de soutien social. Curry et Russ (1985) ont observé les stratégies adaptatives d'enfants âgés de 8 à 10 ans dans une situation stressante non contrôlable (subir un traitement chez le dentiste). Les résultats indiquent une relation positive entre l'âge, ainsi que la fréquence, et la diversité des modes de coping cognitif, la restructuration cognitive positive. D'autres résultats suggèrent une relation négative entre l'âge et la recherche d'informations. Remarquons que, dans ce type de situation, il y a peu de chose à faire, si ce n'est d'utiliser une stratégie cognitive permettant de maintenir un degré relatif de confort psychologique.

\section{LE COPING SOCIAL}

Les stratégies de coping se développent ou se transforment souvent au sein d'une unité sociale significative (la famille, le groupe d'amis, le couple, un groupe de travailleurs). Pour Cartwright et Cooper (1996), la recherche 
d'avis et de soutien social peut être classifiée à la fois comme coping centré sur les émotions (le réconfort reçu peut réduire l'anxiété) et comme coping centré sur le problème (l'aide financière ou l'assistance reçue peut aider à résoudre les problèmes). Le besoin de soutien social selon l'âge (source d'informations et de réconfort) dépend peut-être des changements qu'entraîne le fait de vieillir. Le besoin de se tourner vers les autres en période de détresse peut varier selon la source de soutien social recherchée comme dans sa fréquence, son intensité et sa fonction. De plus, la recherche de soutien social peut être jugée comme un bon coping ou un moins bon coping. En effet, on peut définir le coping social comme un coping basé sur l'attachement et, dans certains cas, comme une stratégie moins avancée au plan développemental, vu la dépendance qu'elle implique parfois envers les autres, surtout durant l'enfance. Bien que cette stratégie de type social soit une façon efficace de faire face à l'adversité, la collaboration interpersonnelle dans les efforts de coping d'une personne peut parfois se révéler contre-productive, surtout si le contexte social n'est pas d'accord avec la réponse de coping de l'individu, si le réseau social ne peut lui venir en aide, si les contextes sociaux sont irresponsables et offrent des modèles de coping inadéquats (Holahan et al., 1996), ou encore si le contexte social minimise la détresse de la personne, ce qui pourrait la décourager dans ses efforts pour faire face à une situation difficile.

Après avoir rencontré des enfants d'âge préscolaire et de deuxième année ainsi que leur mère, Bernzweig, Eisenberg et Fabes (1993) notent, avec l'âge, une diminution dans l'utilisation des stratégies de recherche de soutien social et une augmentation dans l'utilisation des stratégies de restructuration cognitive, d'évitement cognitif et de résolution directe du problème. Quand on regroupe les catégories de soutien social (les stratégies centrées sur le problème et celles centrées sur les émotions), on remarque que les enfants d'âge préscolaire utilisent beaucoup plus de ces stratégies que les plus vieux, comme s'ils étaient conscients qu'ils n'avaient pas encore les ressources et les habiletés suffisantes pour venir en aide à une personne en détresse (mise en situation fictive). D'autres résultats rapportés par Prinstein, La Greca, Vernberg et Silverman (1996) vont dans le même sens. En effet, dans l'inventaire de coping d'enfants de troisième année jusqu'à la septième année scolaire, les plus jeunes adoptent plus un coping d'assistance émotionnelle des parents et des amis que ne le font les enfants de quatrième et cinquième année. Finalement, s'appuyant sur une enquête dans laquelle on identifie les stratégies les plus fréquemment utilisées par des enfants de 8 à 12 ans pour faire face à un stresseur en général, Muir Ryan (1989) constate qu'à huit ans les enfants ont plus souvent recours au soutien social et beaucoup moins à l'exercice physique et aux activités cognitives et émotives que les plus 
vieux. À l'âge de neuf ans, ils utilisent plus les agressions verbales, les activités évitantes et les réponses émotionnelles. À 10 ans, ils font moins souvent référence aux activités évitantes. À 11 ans, ils déploient plus d'activités physiques et sont les moins nombreux à utiliser les agressions verbales et les activités distrayantes. Enfin, à l'âge de 12 ans, les enfants utilisent plus la relaxation, des activités cognitives et de distraction que les plus jeunes.

\section{LE COPING ÉMOTIONNEL}

Certains chercheurs notent une relation positive entre le coping centré sur l'émotion et les enfants âgés de 6 à 12 ans, de 6 à 17 ans (Brotman Band et Weisz, 1988; Compas et Worsham, 1991) et de 10 à 14 ans (Compas et al., 1988). Boekaerts (1994, dans Boekaerts, 1996) propose à des enfants âgés de 10 à 12 ans et des adolescents âgés de 14 à 15 ans deux situations dont l'une comprend un stresseur scolaire (tu es en classe et le professeur veut que tu prennes des notes mais il parle trop vite) et l'autre un stresseur interpersonnel (tu es brutalisé à l'école). Les résultats indiquent que, peu importe l'âge, les élèves utilisent plus le coping centré sur le problème face au stress scolaire jugé contrôlable que face au stress interpersonnel. Par contre, les plus jeunes utilisent moins le coping centré sur l'émotion que les plus vieux face au stress scolaire. Spirito, Stark, Grace et Stamoulis (1991) trouvent, à partir du Kidcope (liste de vérification utilisée pour évaluer les stratégies cognitives et comportementales auprès d'enfants de 9 à 14 ans, présentée dans le chapitre 6), que les plus jeunes (de 9 à 11 ans) utilisent plus de restructuration cognitive, de résolution de problèmes, de régulation des émotions et de pensée magique que les adolescents de 14 ans, peu importe s'il s'agit d'une réponse à un problème typique ou à un problème qu'ils auraient vécu.

En somme, si on peut conclure que le coping cognitif tend à augmenter avec l'âge, les résultats sont moins clairs pour ce qui est du coping social et émotionnel, sans doute à cause des différences d'échantillons, des catégories de coping, des instruments de mesures (liste, entrevue individuelle, observation), des situations de stress (situation contrôlable/ incontrôlable; plaisante/déplaisante; réelle/fictive).

\section{CONCLUSION}

En intervention clinique, l'acquisition de nouveaux modes de coping dépend en partie du degré de maturité de l'enfant (Compas, 1998). Par exemple, il est contre-productif d'enseigner des stratégies de coping si 
l'enfant n'a pas encore développé les ressources et les capacités nécessaires pour les exécuter (la restructuration cognitive peut nécessiter la capacité de penser à des solutions de rechange et à utiliser la pensée hypothétique, ce qui excède les capacités cognitives du jeune enfant). Quand un enfant acquiert de nouvelles compétences, il développe de nouvelles ressources pour mieux faire face à l'adversité. Il devient alors plus apte à répondre efficacement aux nouvelles demandes internes (la puberté, la ménopause) et externes (les pertes relationnelles, matérielles, statutaires). Par contre, si l'acquisition de nouvelles compétences intellectuelles rend plus facile la planification pour l'enfant (l'habileté d'anticipation), elle lui offre en revanche plus d'occasions de s'inquiéter avant le temps et de se sentir menacé (Skinner et Edge, 1998). En effet, si l'habileté d'imaginer de multiples conséquences aide à la résolution de problèmes ou à la restructuration cognitive, cette nouvelle capacité permet aussi d'imaginer plusieurs conséquences négatives à une situation problématique, ce qui peut augmenter le degré d'anxiété. Autrement dit, le développement de la régulation des émotions permet à l'enfant de se réconforter (autocommunication positive) ou, dans certains cas, de se dénigrer et de s'inquiéter. Il semble donc que chaque nouvelle compétence acquise durant le développement offre un gain et une perte dont il faut tenir compte. Par ailleurs, il faut savoir que certaines personnes restent en santé et acquièrent de la maturité plus rapidement en dépit d'une exposition à des circonstances stressantes d'adversité; c'est le cas des enfants résilients. Changer le mode de coping d'un enfant signifie parfois changer le contexte social dans lequel il évolue afin de modifier sa façon de se percevoir et de percevoir le monde (Skinner et Edge, 1998).

On peut conclure ici que la réponse de coping dépend de plusieurs paramètres: les ressources disponibles (personnelles, familiales, environnementales, matérielles), l'histoire de succès/d'échec de certains modes de réaction face aux agents de stress, l'âge, le sexe, le degré de contrôle face à la situation, les facteurs de vulnérabilité (facteurs de risque) et de résistance (facteurs de protection) de la personne ainsi que la fréquence, l'intensité, la durée et le type de stress auquel on doit faire face (famille, école, pairs; Strack et Feifel, 1996). Il importe donc de tenir compte de l'ensemble de ces facteurs dans les études transversales et longitudinales futures. Enfin, les psychologues qui œuvrent auprès des personnes stressées doivent les aider non seulement à éviter ou à minimiser les effets adverses du stress sur leur santé mentale et physique, mais aussi à promouvoir leur bien-être psychologique en situation de stress par l'optimisme et le développement d'un affect positif malgré l'état de crise. 


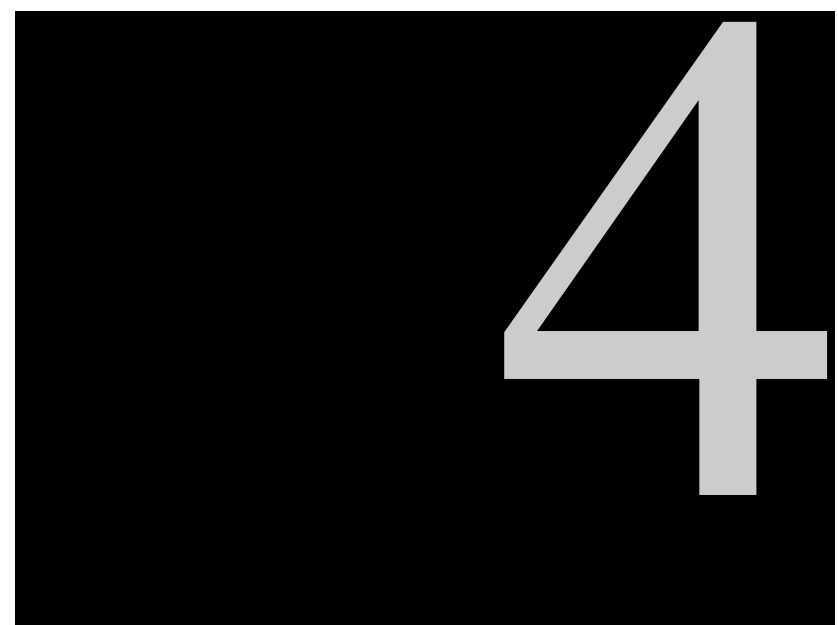

Les strategies a acaptation de la mère et de son enfant dans le phénomène de résilience

\author{
MARC A. PRovost ${ }^{1}$ \\ Département de psychologie \\ Université du Québec à Trois-Rivières \\ Trois-Rivières (Québec)

\section{MICHELLE DUMONT} \\ Département de psychologie \\ Université du Québec à Trois-Rivières \\ Trois-Rivières (Québec)
}

\author{
SYlvain CouTu \\ Département de psychoéducation \\ et de psychologie \\ Université du Québec à Hull \\ Hull (Québec)
NICOLE ROYER Université du Québec à Trois-Rivières Trois-Rivières (Québec) \\ Département des sciences de l'éducation
}

1. Cette recherche a été rendue possible grâce à une subvention du Conseil québécois de la recherche sociale (CQRS) et du Fonds institutionnel de la recherche (FIR) de l'Université du Québec à Trois-Rivières. Les auteurs remercient madame Rollande Lecours de la Commission scolaire des Vieilles Forges (Trois-Rivières) pour sa précieuse collaboration. Nous remercions aussi les éducatrices des maternelles et des garderies ainsi que les directeurs d'école qui nous ont permis de compléter la collecte des données. Toute correspondance doit être adressée à Marc A. Provost, Département de psychologie, Université du Québec à Trois-Rivières, C.P. 500, Trois-Rivières (Québec) Canada G9A 5H7. 



\begin{abstract}
Au cours des dernières décennies, la recherche a fait des progrès appréciables dans l'analyse des facteurs qui augmentent les risques, pour les jeunes enfants, de développer des problèmes de comportements. Au nombre de ces facteurs, citons en particulier les vulnérabilités génétiques, les conditions de vie stressantes comme la pauvreté, les conflits familiaux et les expériences traumatiques comme la perte d'un parent. Cependant, les chercheurs qui ont le plus œuvré dans ce domaine insistent sur le fait qu'il faut bien faire la distinction entre risques d'inadaptation et l'inadaptation per se. En effet, les observations maintes fois répétées ont démontré que, même dans des conditions d'adversité aiguës, les enfants varient beaucoup dans leur expression de l'adaptation, certains démontrant d'excellentes compétences (Wyman, Cowen, Work, Hoyt-Meyers, Magnus, et Fagen, 1999).
\end{abstract}

Le concept de résilience, né de cette constatation que certaines équipes de recherche ont faite en observant des groupes à risques (Garmezy, Masten, et Tellegen, 1984; Rutter, 1987), désigne la capacité d'un enfant de se développer normalement malgré des conditions de vie particulièrement difficiles qui sont généralement considérées comme des facteurs de risque d'inadaptation. L'utilité de ce concept est évidente. La compréhension des processus qui permettent une telle adaptation réussie peut nous aider à mieux comprendre le développement normal et pathologique (Cicchetti et Cohen, 1995) et nous donner la connaissance nécessaire pour entreprendre des interventions préventives.

$\mathrm{Au}$ début de la recherche sur la résilience, les spécialistes cherchaient surtout des caractéristiques exceptionnelles chez les enfants qu'ils identifiaient alors comme des " invulnérables » (Garmezy et Nuechterlein, 1972). De nos jours, les chercheurs tentent plutôt de comprendre la résilience dans le contexte des processus normaux de développement pour tenir compte du continuum de la qualité des adaptations et de l'ensemble des facteurs qui sont en jeu dans le développement. 
Wyman et al. (1999) proposent de considérer la résilience dans le cadre du modèle de la structure organisationnelle du développement proposé par Waters et Sroufe (1983). Selon ces deux auteurs, le processus d'adaptation doit être considéré comme le pivot de tout développement réussi. Cette adaptation est un processus dynamique qui permet à l'enfant d'utiliser ses ressources internes et des éléments de son environnement pour arriver à maîtriser chaque étape de son développement, que Waters et Sroufe (1983) désignent comme les tâches développementales. À chacune de ses étapes, le résultat obtenu reflète le processus transactionnel entre l'enfant et son milieu. Selon les tâches développementales, le rôle $\mathrm{du}$ milieu et des ressources internes de l'enfant peut varier. Par exemple, au cours de la première année, le développement des compétences est en grande partie stimulé par la relation socioaffective avec les parents. L'effet des facteurs de risque sur l'enfant semble alors indirect puisque l'environnement psychosocial de la famille est tamisé par les parents, en particulier la mère. Plus tard, l'adaptation de l'enfant dépend plus des ressources internes qu'il a su développer, de sorte que les facteurs de risque agissent plus directement sur l'enfant.

Wyman et al. (1999) précisent que, dans cette perspective, les comportements adaptatifs de l'enfant ne sont pas synonymes de résilience. Il faut plutôt les considérer comme des comportements acquis qu'il peut utiliser pour promouvoir sa résilience, $c^{\prime}$ est-à-dire une maîtrise adéquate des tâches développementales dans des conditions difficiles peu propices au développement harmonieux d'un individu.

La résilience doit donc pouvoir compter sur des ressources internes et externes qui permettent l'adaptation adéquate. Ces ressources sont souvent considérées comme des facteurs de protection qui atténuent ou éliminent les effets négatifs des facteurs de risque (Cohen et Wills, 1985; Garmezy, 1983). La documentation récente rapporte plusieurs ressources qui prédisent la résilience. Il serait fastidieux de tout passer en revue dans ce chapitre. Citons simplement, pour bien illustrer l'adéquation du modèle de Waters et Sroufe (1983), quelques exemples de facteurs qui reçoivent l'assentiment de la plupart des spécialistes. Chez les très jeunes enfants, Werner et Smith (1992) proposent le tempérament facile qui facilite la capacité de répondre de la mère. Chez les enfants d'âge scolaire, les facteurs de protection se diversifient et deviennent plus complexes. Citons par exemple l'empathie ou les attentes positives à l'endroit du futur (Wyman et al., 1999). Chez les adolescents, l'estime de soi et le soutien social des pairs reviennent à quelques reprises (Dumont et Provost, 1999 ; Plancherel, Bolognini et Nunez, 1994). Inspirés de Lazarus 
et Folkman (1984), Dumont et Provost ajoutent à cette liste les stratégies adaptatives comme facteurs modérateurs du lien stress (risques)-détresse (inadaptation).

Le but de la recherche que nous présentons ici est de vérifier l'importance des stratégies d'adaptation (coping) comme ressources internes des enfants résilients. Suivant le modèle de Waters et Sroufe (1983), nous avons aussi incorporé l'analyse du coping maternel comme marqueur des ressources externes en relation transactionnelle avec les stratégies de l'enfant. Au niveau préscolaire, la tâche développementale est surtout la socialisation de l'enfant. Par contre, la mère est encore importante pour développer des apprentissages chez son enfant. Nous avons donc choisi d'analyser ses propres capacités de stratégies d'adaptation. Ce choix permet, d'une part, de considérer un aspect typique du fonctionnement de la mère en relation avec son enfant et, d'autre part, de voir à quel point les stratégies de la mère, modèle d'identification, peuvent avoir une influence sur celles de son enfant.

Au cours des 15 dernières années, le lien a été clairement établi entre le stress, qui est un indicateur de risque, le coping et la santé mentale et physique. Dans le lien stress-détresse, le coping peut être vu comme un facteur protecteur modérant les effets du stress sur la santé. Nous pouvons donc considérer le coping comme une ressource potentiellement utile au développement de la résilience. L'opérationalisation la plus connue du concept de coping a été proposée en premier par Lazarus en 1966 et développée par la suite dans un ouvrage de référence écrit en collaboration avec Folkman (Lazarus et Folkman, 1984). Ces auteurs définissent le coping comme l'ensemble des efforts cognitifs et comportementaux qui permettent de tolérer, d'éviter ou de minimiser l'effet du stress. Le coping peut être positif ou négatif, actif ou évitant, direct ou indirect. Si les recherches sur le coping ont surtout été faites avec des populations adultes ${ }^{2}$, on observe un intérêt récent pour l'étude de ce phénomène au cours de l'enfance et surtout de l'adolescence (Altshuler et Ruble, 1989; Blanchard-Fields et Coleman-Irion, 1988 Compas, Banez, Malcarne et Worsham, 1991 ; Seiffge-Krenke, 1994a ; 1994b ; Spirito, Stark, Grace, et Stamoulis, 1991). Soulignons en particulier l'étude de Altshuler et Ruble (1989) qui ont noté, chez les enfants de 5 à 12 ans, une augmentation des stratégies centrées sur l'émotion, grâce à leur capacité croissante de verbaliser, de différencier leurs sentiments et de se calmer eux-

2. En effet, d'après une enquête menée par Seiffge-Krenke et Shulman (1993), seulement 7 \% des études effectuées dans les vingt dernières années auraient porté sur le coping chez les jeunes, notamment chez les adolescents. 
mêmes. Ces résultats vont dans le même sens que ceux de Compas et al. (1991) qui ont mis en évidence un coping orienté sur le problème durant l'enfance et sur les émotions au début de l'adolescence. Ils expliquent ce phénomène par les raisons suivantes: 1) le coping centré sur le problème, par rapport à celui sur les émotions, est plus facilement acquis par l'observation des comportements adultes exprimés ouvertement; 2) l'enfant a moins accès ou moins conscience de ses états émotionnels internes; et 3) le jeune ne conçoit pas encore que ses émotions peuvent être soumises à une autorégulation. La documentation sur les très jeunes enfants s'arrête cependant à ces deux études. Au plan de la résilience, aucune étude ne fait appel au coping chez les jeunes.

L'opérationalisation et l'analyse de la résilience peuvent adopter deux perspectives différentes (Masten, Hubbard, Gest, Tellegen, Garmezy, et Ramirez, 1999). Tout d'abord, l'approche centrée sur les variables et leurs possibles covariables dans des modèles de régression ou de méthodes de variables latentes (Masten, 1989; Masten et al., 1999). Une seconde approche centrée sur les groupes d'individus privilégie plutôt la comparaison entre des groupes d'enfants résilients et des groupes d'enfants inadaptés qui ont le même degré de risque que les résilients mais pas leur succès adaptatif. Plus récemment, certains ont ajouté des groupes d'enfants compétents qui ont le même succès adaptatif que les résilients sans en avoir le degré de risque (Dumont et Provost, 1999; Masten et al., 1999|. Cette approche, que nous avons utilisée dans notre recherche, pose cependant le problème de l'identification précise des enfants dans chaque groupe. En effet, il n'y a pas de consensus sur l'utilisation d'un critère universel pour évaluer la résilience, chacun utilisant ses propres critères en fonction de la question posée (Masten et al., 1999). En utilisant cependant le cadre théorique de Waters et Sroufe (1983), nous pouvons poser l'hypothèse que les critères généraux pour les enfants résilients sont qu'ils fonctionnent raisonnablement bien dans les tâches appropriées à leur stade de développement tout en ayant expérimenté des stress importants. Dans notre étude portant sur des enfants fréquentant la garderie ou la maternelle, la tâche développementale particulière à ces enfants est l'acquisition de la compétence sociale (Waters et Sroufe, 1983) et les stress significatifs sont tous liés à des problèmes familiaux (faible niveau socioéconomique, monoparentalité, etc.).

La recherche que nous présentons a pour but d'analyser les stratégies d'adaptation dans des cas de résilience. S'inscrivant dans le modèle transactionnel de Waters et Sroufe (1983), elle insiste sur les stratégies de 
l'enfant (ressources internes) et celles de la mère (ressources externes) comme deux aspects du même phénomène de protection dont semblent jouir les enfants résilients.

\section{MÉTHODE}

\section{LES PARTICIPANTS}

Comme nous l'avons mentionné plus haut, nous avons choisi l'approche centrée sur les groupes (Masten et al., 1999) qui privilégie la comparaison des enfants résilients avec d'autres types d'enfants. Dans ce type de recherche, le choix des participants est crucial. Il est difficile d'établir un schème de recherche classique et bien balancé où tous les groupes sont faciles d'accès. En outre, la documentation scientifique dans ce domaine est relativement rare et peu d'exemples donnent des expériences de choix judicieux. Le groupe du Minnesota (Garmezy et al., 1984; Masten et Garmezy, 1985; Masten et al., 1999) est une des équipes les plus reconnues dans le domaine des familles à risque et leur expertise dans la sélection des participants nous semble la plus adéquate. Nous nous sommes donc inspirés de leur devis de recherche pour notre sélection. Selon cette méthode, les participants sont choisis par étapes, par tamisage d'une population générale en fonction des critères retenus. Ajoutons ici que le problème majeur est celui du dépistage d'enfants qui n'ont pas une courbe développementale attendue (les résilients et ceux qui montrent des signes de troubles tout en provenant de population normale). Le groupe des enfants perturbés était relativement facile à constituer puisque nous avions accès à une population dite à risque. Nous avons donc opté pour la technique du tamisage pour nous assurer que tous nos participants étaient sélectionnés par la même technique d'échantillonnage.

\section{PREMIÈRE ÉTAPE : LE CHOIX DE LA POPULATION}

Les participants ont été sélectionnés dans les services de garderie et de maternelle de la région de Trois-Rivières pour diverses raisons: d'abord, nous y retrouvons des enfants qui ont une expérience de vie sociale parmi des pairs; ensuite, nous pouvons compter sur des intervenantes fiables pour obtenir, pour chacun de ces enfants, un jugement rapide de leur degré d'adaptation à la tâche développementale caractéristique de cet âge (Waters et Sroufe, 1983). Nous avons déjà vérifié les compétences d'observation de ces intervenantes et nous les avons utilisées à plusieurs reprises avec succès (Provost et LaFreniere, 1991; LaFreniere, Provost, et Dubeau, 1992). 
Tous les enfants sont donc d'âge préscolaire et fréquentent assidûment une garderie ou une maternelle. Deux garderies (sur un échantillon de huit) et huit maternelles (sur un échantillon de 12) desservent des quartiers défavorisés où le nombre de familles à risque est plus élevé.

\section{DEUXIÈME ÉTAPE : LE CHOIX DES PARTICIPANTS}

Dans un premier temps, nous avons rencontré des groupes de parents en début d'année pour leur expliquer les buts du projet. La participation du personnel des écoles et des garderies nous a grandement facilité la tâche. Par la suite, nous avons envoyé à toutes les familles une lettre qui expliquait dans les grandes lignes le projet et demandait aux parents de remplir un questionnaire sur les événements stressants de la vie inspiré des travaux classiques de Holmes et Rahe (1967). Ces derniers affirment en effet avoir eu de très bons résultats avec un questionnaire simple sur le stress de la vie quotidienne qu'il considèrent comme le premier facteur de risque, même si ce facteur est en fait la résultante d'autres facteurs (niveau socioéconomique, bas par exemple).

Nous y avons ajouté une série de questions simples afin de cerner rapidement le stress familial, comme le niveau socioéconomique et le statut matrimonial de la famille (biparentale, monoparentale, reconstituée). Il y a en effet plusieurs facteurs de risque recensés dans la documentation scientifique. Cependant, selon Garmezy et al. (1984), les principaux facteurs sont le stress, la monoparentalité et le faible niveau socioéconomique. Pas moins de 120 familles se sont montrées intéressées à participer à notre projet.

\section{LES QUESTIONNAIRES POUR LA FORMATION DES GROUPES}

Une fois ce premier échantillon constitué, il s'agissait de déterminer dans quel groupe situer chaque enfant. La catégorisation des quatre groupes provient de la définition même de la résilience: un enfant qui semble se développer normalement malgré des signes d'adversité dans son environnement. Nous avons donc déterminé l'appartenance aux groupes en accordant un score de fonctionnement social (le profil socioaffectif: PSA) et un score d'adversité familiale (le stress familial).

Le profil socioaffectif (PSA). Nous avons fait appel aux éducatrices pour remplir le profil socioaffectif (PSA), un instrument adapté aux enfants d'âge préscolaire (30 à 72 mois) qui permet de cerner leurs difficultés et leurs compétences socioaffectives (LaFrenière, Dubeau, Janosz et Capuano, 1990). Le questionnaire est composé de 80 énoncés qui décrivent des comportements de l'enfant. Ces 80 énoncés sont divisés en 
huit échelles (les $\alpha$ variant de 0,79 à 0,91 selon les échelles; la stabilité de 0,79 à 0,87 selon les échelles). Trois échelles caractérisent l'expression des émotions en général, trois autres fournissent de l'information sur les interactions de l'enfant avec ses pairs et les deux dernières touchent les interactions de l'enfant avec les adultes de la garderie. Chacune des échelles comporte un pôle positif et un pôle négatif (chacun composé de cinq énoncés). Un score est calculé pour chacune de ces échelles. On peut aussi calculer un score d'externalisation et un score d'internalisation, de manière à rencontrer les concepts les plus fréquemment utilisés dans le domaine. Nous obtenons également un score global de l'adaptation sociale de l'enfant à la garderie et à la maternelle. L'outil a été normalisé sur une population québécoise et procure des profils de comportement pour chaque enfant en fonction des normes établies de façon indépendante pour les garçons et les filles.

Selon les études de LaFreniere (LaFreniere et al., 1990), un score de un écart type et plus au-dessus de la moyenne de l'échelle d'internalisation ou d'externalisation constitue un indice important et fiable de problèmes de comportement. Nous avons donc choisi ce critère pour déterminer nos groupes: les enfants ayant un score de un écart type et plus au-dessus de la moyenne de l'échelle d'internalisation ou d'externalisation sont considérés comme les enfants ayant effectivement des problèmes de comportements sociaux alors que ceux qui ont un score de un écart type et plus au-dessous de la moyenne de l'échelle d'internalisation ou d'externalisation sont considérés comme des enfants qui n'ont pas de problèmes d'adaptation.

Les conditions sociales de la dyade: le stress familial. Nous avons retenu six indices de stress familial. Chacun de ces indices est dichotomisé, c'est-à-dire que la présence du stress est cotée 1 et l'absence est cotée 0. La somme des indices donne la cote de stress familial. Nous avons choisi nos indicateurs de stress en nous basant sur la documentation existante (Holmes et Rahe, 1967; Vitaro, Gagnon, et Tremblay, 1992). Les indices sont les suivants: la monoparentalité, le revenu familial (en bas de $20000 \$$ avec 1 enfant $=1 ; 30000 \$$ et 2 enfants $=1$, et ainsi de suite), l'âge de la mère à la naissance du premier enfant (moins de 22 ans $=1$ ). Le questionnaire sur les événements stressants de la vie nous permet de noter la présence de stress liés à la santé, au travail (chômage, baisse de salaire, etc.) et aux relations familiales (mortalité dans la famille, hospitalisation d'un membre de la famille, etc.). Selon Rutter (1987), une famille qui déclare plus de deux stress majeurs doit être considérée comme à risque. Nous avons donc choisi ce critère pour distinguer les familles à risque et les familles sans risque. 


\section{LA FORMATION DES QUATRE GROUPES}

Nous avons formé les quatre groupes en appliquant les deux critères (adaptation sociale et risques) aux 120 familles de notre échantillon. Nous en somme arrivés aux quatre groupes suivants;

a) Les familles vulnérables. Ce groupe comporte 23 familles dont la cote au stress est supérieure ou égale à 2 (donc deux stress en montant) et dont l'enfant présente un problème d'internalisation ou d'externalisation (un écart type au-dessus de la moyenne sur un de ces deux scores).

b) Les familles compétentes. Ce groupe comprend 26 familles dont la cote au stress est inférieure à 2 et dont l'enfant ne présente aucun problème d'adaptation (un écart type au-dessous de la moyenne pour les échelles d'internalisation ou d'externalisation).

c) Les familles résilientes. Ce groupe comporte 16 familles dont la cote au stress est supérieure ou égale à 2 (donc deux stress en montant) et dont l'enfant ne présente aucun problème d'adaptation (un écart type au-dessous de la moyenne pour les échelles d'internalisation ou d'externalisation).

d) Les familles aberrantes. Ce groupe comprend 21 familles dont la cote au stress est inférieure à 2 et dont l'enfant présente un problème d'internalisation ou d'externalisation (un écart type au-dessus de la moyenne sur un de ces deux scores).

Cette sélection des participants a été effectuée lorsque les enfants avaient entre trois et six ans. Les données sur les stratégies d'adaptation ont été recueillies deux ans plus tard à l'intérieur d'un projet longitudinal. Les mesures que nous présenterons maintenant ont été prises au moment où les enfants avaient entre cinq et sept ans. En effet, les stratégies d'adaptation ne peuvent être mises en lumière à un si jeune âge et il faut attendre le début de la période scolaire pour pouvoir les analyser. Nous avons pris soin de vérifier la stabilité de nos groupes. Toutes les familles qui ont accepté de collaborer à nouveau ont donc rempli le questionnaire de stress et les professeurs, le questionnaire d'Achenbach (Achenbach et Edelbrock, 1981) qui permet de déceler les problèmes d'internalisation et $d^{\prime}$ externalisation chez les enfants d'âge scolaire ${ }^{3}$. Les corrélations entre les échelles d'internalisation et d'externalisation des deux questionnaires,

3. Nous n'avons pas utilisé le PSA ici puisque ce questionnaire a été conçu pour des enfants d'âge préscolaire. 
sur l'ensemble des enfants que nous avons pu réévaluer, sont significatives (externalisation: $r(55)=0,71, p<, 001$; internalisation: $r(55)=0,32$, $p<0,01$, ce qui nous permet de considérer que nous touchons aux mêmes concepts. En outre, tous les enfants ont conservé des scores au stress familial et à l'Achenbach qui les placent dans le même groupe que deux ans auparavant. Par contre, nous avons perdu plusieurs enfants de l'échantillon initial (certaines familles ont refusé de continuer, d'autres avaient quitté la région). L'échantillon final utilisé dans l'analyse des résultats que nous présenterons plus bas contient 9 enfants aberrants, 8 enfants vulnérables, 13 enfants compétents et 12 enfants résilients.

\section{LES INSTRUMENTS DE MESURE}

Stratégies d'adaptation (coping). Le Coping pour les adultes. (Ways of Coping Questionnaire, de Folkman et Lazarus, 1988; validation de la version abrégée par Bouchard, Sabourin, Lussier, Richer et Wright, 1995). Ce questionnaire évalue les stratégies d'adaptation employées par les individus pour faire face aux situations stressantes. Une traduction française avec procédure inversée fut réalisée par Mishara (1987). Dans cette étude, nous utilisons une version courte comptant 21 items (Bouchard et a1., 1995). Ces items sont cotés sur une échelle à quatre points ("pas utilisé ", "utilisé de temps en temps ", "utilisé souvent » et "beaucoup utilisé»| couvrant des stratégies comportementales ou cognitives. Les qualités métriques de la version courte révèlent, auprès d'un échantillon de 506 couples, une structure factorielle tridimensionnelle (recherche de soutien social, réévaluation positive-résolution de problèmes, distanciationévitement). Les analyses de cohérence interne révèlent des alphas de 0,79 pour le premier facteur, de 0,77 et de 0,82 pour les second et troisième facteurs (Bouchard et al., 1995).

Le Coping pour les enfants. À partir des études de Altshuler et Ruble (1989) et de Brotman Band et Weisz (1988), nous avons conçu une entrevue structurée contenant sept scénarios hypothétiques, présentés sous forme d'images, chacune décrivant un enfant (un garçon dans le cas d'une entrevue avec un garçon et une fille dans le cas d'une entrevue avec une fille) vivant une situation stressante relativement incontrôlable. Deux histoires présentent une expérience stressante en situation positive (la frustration engendrée par l'attente d'ouvrir un cadeau placé sous le sapin quelques jours avant Noël; la première journée à l'école). Les autres histoires décrivent des situations stressantes en situation négative (attendre dans le cabinet du médecin pour recevoir un vaccin, se faire mal en tombant de son vélo, perdre un ami qui déménage dans un autre quartier, 
recevoir des insultes d'un autre enfant, se faire gronder pour avoir brisé un objet). Une fois le scénario décrit, l'entretien individuel se déroule en deux temps. Premièrement, on demande à l'enfant comment le protagoniste devrait se sentir dans la situation. Deuxièmement, on dit à l'enfant que le protagoniste ${ }^{4}$ se sent effrayé ou excité selon 1'histoire. On lui demande alors: 1) Penses-tu qu'il pourrait se sentir moins effrayé (ou excité)? 2) Qu'est-ce qu'il peut faire par rapport au fait d'être effrayé (ou excité)? On encourage les réponses des enfants en reformulant chaque question trois fois. Par exemple, en changeant la situation $\left(\mathrm{Qu}^{\prime}\right.$ est-ce qu'il pourrait faire pour changer ce qui est arrivé?), en modifiant la cognition (Qu'est-ce qu'il pourrait faire pour changer sa manière de penser?) et en transformant l'émotion (Qu'est-ce qu'il pourrait faire pour changer ses sentiments à propos de cela?).

\section{LES PROCÉDURES}

Nous l'avons déjà indiqué, ce projet s'inscrit dans le cadre d'une étude longitudinale. La formation des groupes s'est effectuée lorsque les enfants avaient de trois à cinq ans. La seconde étape, celle de l'analyse spécifique des stratégies d'adaptation, s'est faite au moment où les enfants avaient de cinq à sept ans.

Dans cette deuxième étape, nous avons rencontré les familles individuellement. Une assistante prenait rendez-vous avec la famille à un moment propice pour la famille, généralement en début de soirée, avant le coucher de l'enfant. Une fois sur place, elle présentait le questionnaire Ways of Coping Questionnaire (Folkman et Lazarus, 1988) à la mère. Étant donné que, dans certaines familles de notre échantillon, les mères comprenaient moins bien les questions, nous avons aidé toutes les mères à répondre au questionnaire pour nous assurer de l'homogénéité dans la compréhension du document. L'assistante se ménageait un peu de temps pendant la visite pour rencontrer l'enfant et lui faire passer le questionnaire sur le coping. Les visites prenaient en général deux heures.

Parallèlement à ces visites, une assistante a rencontré tous les enseignants de ces enfants pour leur distribuer et leur expliquer le questionnaire d'Achenbach. Elle remettait à chaque professeur une enveloppe affranchie qu'il devait retourner par la poste.

4. Pour alléger le texte, nous retiendrons ici le masculin, en précisant toutefois que lors des entrevues le genre des personnages correspondait au sexe de l'enfant. 


\section{LES RÉSULTATS}

Cette section comprend deux volets. Dans le premier, nous présenterons la conversion des différents questionnaires en scores d'analyse et dans le second, les résultats des diverses analyses pour les quatre groupes.

\section{LA RÉDUCTION DES DONNÉES}

Voici comment nous avons transformé les données brutes pour en extraire nos indicateurs de mesure.

\section{LES STRATÉGIES D’ADAPTATION DE LA MÈRE (COPING)}

Ce questionnaire de Lazarus et Folkman (1984) comporte 21 questions avec choix de réponses de type Likert. Il distingue trois dimensions des stratégies d'adaptation. La première échelle a trait aux stratégies de recherche de soutien et comprend six questions. Un score élevé à cette échelle indique que la mère a tendance à rechercher le soutien de son milieu pour solutionner ses problèmes. La seconde échelle, de six questions, touche la dimension d'évitement. Un score élevé à cette échelle signifie que la mère a plutôt tendance à éviter les problèmes qu'elle rencontre. Finalement, la troisième échelle, de neuf questions, mesure la capacité de la mère à faire face aux situations (résolution de problèmes). Un score élevé à cette échelle signifie que la mère préfère affronter les problèmes et $\mathrm{y}$ trouver une solution personnelle.

\section{LES STRATÉGIES D'ADAPTATION DE L'ENFANT (COPING)}

L'entrevue semi-structurée que l'expérimentatrice fait avec l'enfant est enregistrée sur un magnétophone portatif puis retranscrite. Cinq membres du laboratoire ${ }^{5}$ ont dressé une taxonomie basée sur les travaux de Altshuler et Ruble (1989), Brotman Band et Weisz, (1988) et Lazarus et Folkman (1984). Cette taxonomie permet de coter chaque réponse de l'enfant selon des types de stratégies d'adaptation (voir le tableau en annexe). Par la suite deux assistantes de recherche ont coté l'ensemble des réponses des enfants en gardant un taux d'accord de $89 \%$.

5. Michelle Dumont, Nicole Royer, Marc Provost et les deux assistantes de recherche, Louise Bourassa et Elsa Beauchesne. 
Les enfants avaient la possibilité de donner, pour chaque scénario, autant de suggestions qu'ils le désiraient. Nous avons donc, par la suite, calculé le pourcentage de réponse pour chaque catégorie, pourcentage que nous avons transformé en arc sinus, ce qui est la procédure à utiliser lorsque la variable dépendante est une proportion (Brotman Band et Weisz, 1988, Kirk, 1982).

\section{LES RÉSULTATS D’ANALYSE}

Le tableau 1 présente la moyenne et les écarts types obtenus pour chacune des deux variables. On peut le constater à la lecture de ce tableau, peu importe le groupe d'appartenance, les stratégies d'évitement, tant chez les mères que chez les enfants, sont nettement les moins utilisées. En outre, les enfants résilients ont une proportion de solution d'approche supérieure aux autres groupes et une proportion d'évitement inférieure à la moyenne.

Ces résultats proposent essentiellement l'analyse de variance simple entre les quatre groupes pour chaque variable. Compte tenu du nombre restreint d'enfants, nous ne pouvions en effet considérer l'analyse de variance multivariée. Nous avons complété chaque analyse par trois contrastes a posteriori qui comparent les enfants résilients aux autres groupes.

Le tableau 2 présente un résumé des cinq analyses de variance et de leurs contrastes a posteriori. La première constatation est que seule l'analyse de variance sur la capacité de résolution de problème de la mère atteint un seuil significatif $(F 3,39=3,73, p<, 01)$. Ce résultat s'explique en partie par le fait que la division de notre échantillon en quatre groupes a engendré de petits nombre d'enfants dans chacun, de sorte que la variance, bien qu'homogène selon les vérifications statistiques que nous avons pris soin de faire, reste petite.

Par contre, plusieurs contrastes atteignant ce seuil significatif, il ressort que la comparaison spécifique des résilients avec chacun des autres groupes révèle des différences marquées. Un examen de ces résultats indique un premier résultat inattendu: les mères des enfants résilients se distinguent avant tout des mères des enfants compétents. En effet, les comparaisons entre ces deux groupes sont significatives pour les trois stratégies d'adaptation des mères. Les mères des enfants résilients recherchent plus de soutien $(M=17,50$ et $M=13,07 ; t=2,01, p<, 05)$, ont plus recours à la résolution de problème $(M=20,83$ et $M=16,83$; $t=2,44, p<, 01)$ et évitent moins les problèmes $(M=2,33$ et $M=2,92$; $t=2,07, p<, 05$ ) que les mères des enfants compétents. Le tableau 2 révèle aussi que les mères des enfants résilients ont également plus recours à la 
résolution de problème que les mères des enfants à haut risque $(M=20,83$ et $M=15,00 ; t=3,12, \mathrm{p}<, 01)$ et celles des enfants aberrants $(M=20,83$ et $M=17,16 ; t=2,05, p<, 05)$.

Pour ce qui est des stratégies d'adaptation des enfants, le tableau 2 n'indique qu'une seule différence: les enfants résilients proposent plus de stratégies d'approche que ne le font les enfants aberrants $(t=1,99, p<, 05)$.

\section{TABLEAU 1}

\section{Moyenne et écart type pour chaque variable}

\begin{tabular}{|c|c|c|}
\hline Variable & Moyenne & Écart type \\
\hline Mère & & \\
\hline $\begin{array}{l}\text { Soutien social } \\
\text { Aberrants } \\
\text { Haut risque } \\
\text { Compétents } \\
\text { Résilients } \\
\text { Moyenne de l'échantillon }\end{array}$ & $\begin{array}{l}14,62 \\
13,95 \\
13,07 \\
17,50 \\
14,84\end{array}$ & $\begin{array}{l}6,54 \\
5,76 \\
4,85 \\
5,05 \\
5,56\end{array}$ \\
\hline $\begin{array}{l}\text { Résolution de problème } \\
\text { Aberrants } \\
\text { Haut risque } \\
\text { Compétents } \\
\text { Résilients } \\
\text { Moyenne de l'échantillon }\end{array}$ & $\begin{array}{l}17,16 \\
15,00 \\
16,83 \\
20,83 \\
17,69\end{array}$ & $\begin{array}{l}5,85 \\
3,02 \\
3,87 \\
3,32 \\
4,49\end{array}$ \\
\hline $\begin{array}{l}\text { Évitement } \\
\text { Aberrants } \\
\text { Haut risque } \\
\text { Compétents } \\
\text { Résilients } \\
\text { Moyenne de l'échantillon }\end{array}$ & $\begin{array}{l}3,33 \\
2,25 \\
2,92 \\
2,33 \\
2,71\end{array}$ & $\begin{array}{l}1,87 \\
2,12 \\
2,72 \\
1,37 \\
2,07\end{array}$ \\
\hline Enfant & & \\
\hline $\begin{array}{l}\text { Approche } \\
\text { Aberrants } \\
\text { Haut risque } \\
\text { Compétents } \\
\text { Résilients } \\
\text { Moyenne de l'échantillon }\end{array}$ & $\begin{array}{l}1,87 \\
2,14 \\
2,12 \\
2,15 \\
2,08\end{array}$ & $\begin{array}{l}0,30 \\
0,46 \\
0,31 \\
0,21 \\
0,33\end{array}$ \\
\hline $\begin{array}{l}\text { Évitement } \\
\text { Aberrants } \\
\text { Haut risque } \\
\text { Compétents } \\
\text { Résilients } \\
\text { Moyenne de l'échantillon }\end{array}$ & $\begin{array}{l}1,03 \\
0,81 \\
0,85 \\
0,81 \\
0,87\end{array}$ & $\begin{array}{l}0,33 \\
0,31 \\
0,33 \\
0,21 \\
0,30\end{array}$ \\
\hline
\end{tabular}




\section{TABLEAU 2}

\section{Résumé des analyses de variances}

\begin{tabular}{lllll}
\hline \multicolumn{1}{c}{ Variables } & $\mathbf{F}$ & Contraste 1 & Contraste 2 & Contraste 3 \\
\hline Mère & & $2,01^{\star}$ & 1,47 & 1,19 \\
$\begin{array}{l}\text { Soutien social } \\
\begin{array}{l}\text { Résolution } \\
\text { de problème }\end{array}\end{array}$ & 1,47 & $2,74^{\star \star \star}$ & $3,12^{\star \star}$ & $2,05^{\star}$ \\
Évitement & 2,28 & $2,07^{\star}$ & 0,42 & 1,5 \\
\hline Enfant & & & & \\
Approche & 1,65 & 0,30 & 0,02 & $1,99^{\star}$ \\
Évitement & 1,17 & 0,37 & 0,01 & 1,66 \\
\hline
\end{tabular}

${ }^{\star \star} p<, 01 ;{ }^{\star} p<, 05$

Contraste 1 : compétents vs résilients

2: haut risque vs résilients

3 : aberrants vs résilients

\section{DISCUSSION}

Notre projet de recherche visait essentiellement à comparer les stratégies d'adaptation des enfants et des mères dans des familles résilientes, vulnérables, aberrantes et compétentes. Pour établir ces comparaisons, nous avons adopté une démarche méthodologique reconnue: nous avons mis en opposition quatre groupes de familles qui correspondent à des profils différents tant dans le fonctionnement social de l'enfant (internalisation, externalisation) que dans le degré de stress qu'elles subissent. À partir des réponses des éducatrices au questionnaire sur le profil socioaffectif de l'enfant (PSA) et des mères au questionnaire sur l'adversité familiale, nous en sommes venus à isoler les quatre groupes suivants: les familles vulnérables, les familles compétentes, les familles résilientes et les familles aberrantes.

Ce type de recherche sur des populations spécifiques à risque est essentiel, d'une part, pour comprendre les processus de développement selon une approche psychopathologique développementale transactionnelle, et, d'autre part, pour mettre au point des programmes d'intervention particuliers qui peuvent aider de façon efficace les populations visées. 
En effet, certains ont pu démontrer que les vulnérabilités et les facteurs de protection peuvent varier d'une condition de risque à l'autre. En outre, Richters et Weintraub (1990) ont noté avec justesse que les chercheurs peuvent définir un groupe à risque bien que certains individus dans ce groupe ne soient pas directement touchés par les expériences stressantes associées aux risques. Dans ce cas, on ne peut pas considérer ces individus comme résilients. Pour Richters et Weintraub (1990), il faut bien prendre soin de vérifier si tous les membres des groupes à risque ont bien été en contact avec les stress à l'étude. Notre approche, qui tient compte d'un certain nombre de facteurs d'adversité dans le milieu familial, nous permet de considérer si les enfants de l'échantillon ont été touchés directement ou indirectement par les stress vécus par leur famille.

Notre échantillon ne comportait que des familles dont l'enfant était d'âge préscolaire au moment de la sélection. Ce choix s'explique facilement. Nous devons absolument étudier les processus de développement de la résistance dès le plus jeune âge, d'une part, pour comprendre les racines mêmes des facteurs qui réussissent à protéger ces enfants pourtant à risque et, d'autre part, pour concevoir des interventions préventives qui ne seront vraiment efficaces qui si elles visent des enfants d'âge préscolaire (Cowen, Work, et Wyman, 1997). Le but, à moyen terme, est de fournir des indications précises pour mettre au point des méthodes d'intervention ciblées sur les facteurs qui ressortiront comme les plus susceptibles de favoriser les capacités des jeunes enfants à se prémunir contre des conditions familiales et environnementales défavorables à leur développement harmonieux.

Notre projet contenait aussi une innovation: nous avons ajouté un groupe de familles dites aberrantes, où les enfants semblaient démontrer des difficultés même si leur famille ne semblait pas touchée par des facteurs de risque décelables. La plupart des études n'avaient jusqu'alors envisagé que de comparer les familles compétentes aux familles résilientes et vulnérables. Cet ajout va bien dans le sens des substrats théoriques de la psychopathologie développementale qui conçoit l'étude du développement en fonction des lignes de développement normales et pathologiques. Selon cette approche, il faut situer le développement pathologique en fonction de la normalité et tenter de saisir la dynamique de la normalité par comparaison avec les développements moins adéquats. Dans cette optique, l'étude de la résilience permet de considérer à la fois les facteurs potentiels de risque, qui augmentent la probabilité des problèmes ultérieurs, et les facteurs compensatoires qui, au contraire, font baisser cette probabilité 
(Cicchetti et Cohen, 1995). En outre, une étude longitudinale permettrait, selon ce modèle, de vérifier les facteurs stables et les facteurs transitoires de la résilience.

\section{LES STRATÉGIES D'ADAPTATION DE LA MÈRE}

Nos résultats indiquent que les mères des enfants résilients recherchent plus de soutien social que ne le font les mères des enfants compétents. Il faut cependant signaler ici que les mères des enfants résilients ont un score nettement plus élevé que les mères des trois autres groupes, même si une seule comparaison atteint le seuil significatif. Les mères des enfants résilients, confrontées à l'adversité familiale, seraient donc capables de rechercher du soutien pour elles et leur famille. Or, les recherches sur le stress et le soutien le démontrent amplement (Provost, 1995), le soutien social est un des facteurs de protection principaux contre les effets du stress. La recherche de soutien par les mères des enfants résilients permet sans doute de vivre le stress de façon moins aiguë, ce qui aurait pour effet de protéger les enfants, qui ont alors la chance de pouvoir se développer adéquatement. Ces mères vont donc chercher plus de soutien que les mères des enfants compétents, qui n'ont peut-être pas besoin d'utiliser cette stratégie puisque leur niveau d'adversité est moindre. Les mères des enfants résilients semblent chercher plus de soutien que les mères des enfants vulnérables qui ont pourtant un niveau d'adversité équivalent.

Les mères des enfants résilients se démarquent particulièrement dans leur stratégies de résolution de problèmes, où elles présentent des scores significativement supérieurs aux mères des trois autres groupes. Par la définition même de leur groupe, on peut facilement imaginer que les mères des enfants résilients font face à plus de problèmes que les mères des enfants compétents. Par contre, le résultat le plus instructif est la différence marquée entre les mères des enfants résilients et les mères des enfants vulnérables, qui ont toutes deux des niveaux d'adversité comparable. Ce résultat semble tout à fait conforme aux attentes. En effet, plusieurs études ont démontré que les mères qui se trouvaient dans une situation stressante avaient des difficultés face aux événements quotidiens. Ainsi, Capaldi et Patterson (1990) et Forehand, Thomas, Wierson, Brody et Fauber (1990) ont observé que les mères nouvellement divorcées avaient des déficits dans leurs habiletés parentales, y compris dans leur capacité de résoudre des problèmes.

Ces résultats semblent bien correspondre aux attentes, puisque l'on peut facilement suggérer que les mères des enfants résilients, devant l'adversité, qui est une caractéristique de ce groupe, sont capables de faire 
face aux problèmes qu'elles rencontrent. Cette capacité peut avoir deux conséquences. D'une part, sachant résoudre les problèmes qu'elles rencontrent, elles agissent comme écran protecteur pour leur enfant, qui n'a pas à subir les conséquences de ces stress répétés et peut donc se développer normalement, d'où son insertion dans le groupe des résilients. D'autre part, ces mères peuvent aussi agir comme exemple pour leurs enfants qui développent eux mêmes ces formes de stratégies. Ils sont donc capables de faire face à certains problèmes sans développer pour autant des troubles du comportement apparents.

Le résultat voulant que les mères des enfants résilients utilisent plus les stratégies d'évitement que les mères des enfants compétents semble a priori plus difficile à expliquer. Toutefois, Lazarus et Folkman (1984), considèrent que les stratégies d'évitement peuvent parfois être appropriées. En effet, selon eux, devant des situations insolubles, il est quelquefois préférable de ne pas chercher de solutions, mais de tenter d'éviter le problème. Dans ce cas, l'individu qui évite la situation fait preuve de jugement en acceptant que la situation le dépasse et qu'il n'y peut rien. Les mères des enfants résilients sont aux prises avec des situations familiales adverses complexes. Nous pouvons donc envisager que ces mères ont appris à bien évaluer les situations sur lesquelles elles avaient prise et celles qu'elles ne pouvaient modifier aisément (monoparentalité, faiblesse du revenu familial). Le fait qu'elles soient capables d'assumer leur incapacité de modifier des situations peut en quelque sorte faire baisser leur stress et les aider à garder une organisation familiale chaleureuse envers les enfants. On peut aussi penser que cette capacité d'évitement serait liée à l'aptitude qu'ont ces mères de s'accorder des périodes de répit, ce qui peut également les aider à faire face plus adéquatement aux stress imposés dans leur vie quotidienne.

\section{LES STRATÉGIES D'ADAPTATION DES ENFANTS}

Du côté des enfants, les résultats sont pour le moins mitigés. En effet, seule une comparaison atteint le niveau significatif. Les enfants résilients utilisent plus les stratégies d'approche que les enfants aberrants. Il est difficile ici d'avancer une explication, puisque le groupe des enfants aberrants est un groupe difficile à définir, à tel point que Masten et al. (1999) préfèrent ne travailler qu'avec les trois autres groupes. 
Il faut aussi souligner le manque de différence dans ce type de stratégies entre les enfants résilients et les compétents. En effet, ce résultat négatif est en lui-même un bon indice que les enfants résilients sont effectivement des enfants qui fonctionnent adéquatement, tout comme les enfants compétents.

Finalement, il faut souligner que les enfant aberrants utilisent plus les stratégies d'évitement que ceux des trois autres groupes. Cette différence $n^{\prime}$ est pas significative, mais on peut $s^{\prime} y$ attarder quelque peu. Les enfants aberrants sont définis comme ceux qui, sans marque d'adversité familiale apparente, développent un profil socioaffectif mal adapté à leur groupe. Dans notre échantillon restreint, la plupart de ces enfants ont obtenus des scores importants d'internalisation. Ce sont des enfants que les éducatrices considèrent comme retirés du groupe. Il y a donc déjà, dans ce profil socioaffectif, une composante d'évitement du groupe. On peut en conclure que ces enfants ont comme fonctionnement général de fuir les situations stressantes, ce qui se reflète dans leurs réponses lors de l'entrevue.

Le manque de résultats significatifs chez les enfants pose le problème de l'interprétation des réponses de ces derniers. D'une part, aucune recherche n'avait jusqu'à présent utilisé des enfants si jeunes pour étudier les bases développementales des stratégies d'adaptation. Les études de Altshuler et Ruble (1989) et de Brotman Band et Weisz (1988), sur lesquelles nous nous sommes appuyés pour établir notre entrevue et notre taxonomie, utilisaient des enfants de niveau primaire, jusqu'à 12 ans. Les enfants de notre échantillon avaient peut-être du mal à bien saisir les situations, à se les représenter et à penser des solutions au moment de l'entrevue.

\section{LES IMPLICATIONS POUR L'INTERVENTION}

Le soucis de l'intervention a été présent dès le début de ce projet de recherche. En fait, la documentation sur les facteurs de risque a toujours pour intérêt l'intervention psychosociale. Amasser des données sur ce qui prédispose les individus à plus ou moins bien se développer procure toujours de l'information pour les intervenants qui cherchent sans cesse des moyens de faire baisser les risques auprès des familles en difficulté. Le travail sur la résilience ajoute à cette information des indications sur les facteurs qui semblent protéger les individus, les facteurs que les intervenants ont donc tout intérêt à développer et à encourager. Les résultats obtenus donnent aussi des munitions aux tenants de la prévention, laquelle fait largement appel aux concepts de risque et de protection qui peuvent se trouver en amont des problèmes de comportement reconnus. Devant 
ces concepts, les intervenants peuvent maintenant s'engager à réduire les facteurs de risque et à augmenter les facteurs de protection pour enrayer les processus de développement des comportements dysfonctionnels.

La recherche a encore beaucoup à explorer. Il faut encore des études sur le développement normal et les risques avant de mettre au point et d'évaluer des programmes d'intervention. Il faut encore amasser de l'information sur les facteurs distaux (classes sociales, quartier, etc.) pour identifier précisément les populations à risque; il faut aussi faire des efforts pour intervenir sur les facteurs proximaux (la famille, l'école, les pairs) dont on sait qu'ils peuvent avoir un effet direct sur l'adaptation psychosociale des jeunes enfants.

Finalement, il faut absolument investir dans l'évaluation de programmes concrets. En effet, les recherches, qu'elles soient longitudinales ou transversales, ne donnent finalement que des résultats de type corrélatif. Notre étude est de ce type. Nous ne pouvons donc que donner des liens de relation entre les facteurs, sans préciser les causes ou les intrants de ces facteurs, et nous ne pouvons que dire que nos données suggèrent le modèle de risque que nous avons adopté. En mettant au point des projets de prévention directement basés sur les modèles théoriques de risque et de protection que la recherche sur la résilience propose et en évaluant chacun des aspects pour établir un bilan, l'intervention devient un test important du modèle de risque, et permet en tout dernier lieu de concevoir des méthodes de prévention efficaces. 


\section{ANNEXE}

\section{Catégories et cotes pour les entrevues de coping-enfant}

\begin{tabular}{|c|c|c|c|}
\hline Cotes & Classe & Catégorie & Sous-catégorie \\
\hline 111 & $\begin{array}{l}\text { Approche centrée } \\
\text { sur le problème }\end{array}$ & comportement & social \\
\hline 112 & & & matériel \\
\hline 121 & & cognition & $\begin{array}{l}\text { autocommunication } \\
\text { positive }\end{array}$ \\
\hline 122 & & & réflexion \\
\hline 123 & & & $\begin{array}{l}\text { recherche } \\
\text { d'information }\end{array}$ \\
\hline 131 & & soutien social & émotif \\
\hline
\end{tabular}

$210 \quad$ Évitement centré comportement

sur les émotions

221 cognition penser à autre chose

231 réduction comportement

240 manipulation

affective

251 fuite purement fuir

252 fuite argumenter

261 négation purement nier

262 négation par compensation

270 dédramatisation

\begin{tabular}{ll}
\hline 300 & Passivité \\
\hline 400 & $\begin{array}{l}\text { Stratégies } \\
\text { dysfonctionnelles }\end{array}$ \\
\hline 500 & Non pertinent \\
\hline
\end{tabular}




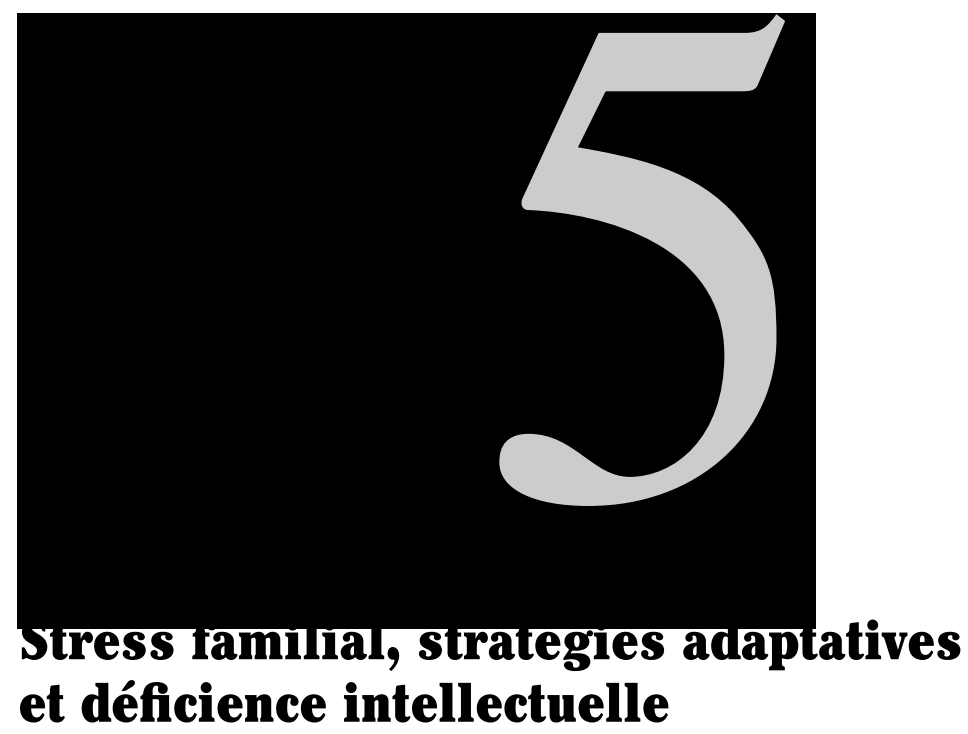

JEAN-LUC LAMBERT

Département de pédagogie curative et spécialisée Université de Fribourg

Fribourg (Suisse)

ROMAIN LANNERS

Département de pédagogie curative et spécialisée Université de Fribourg

Fribourg (Suisse) 

Que signifie être le père ou la mère d'un enfant déficient intellectuel? S'agit-il d'une épreuve insurmontable qui perturbe durablement l'existence des personnes touchées? Quels éléments les professionnels de la psychologie et de la pédagogie peuvent-ils apporter pour aider ces familles dans l'éducation de leur enfant? Telles étaient les questions que se posaient, il y a bientôt trente ans, les chercheurs confrontés à des familles ayant un enfant déficient intellectuel. Trop souvent démunis de points de repère en raison notamment $d$ 'une formation insuffisante, ces chercheurs trouvaient peu de réponses dans les ouvrages scientifiques de l'époque, l'éducation familiale dans son ensemble n'en étant qu'à ses premiers balbutiements. Plus tard, nous comprîmes que cette carence de données scientifiques était en fait le reflet d'une mentalité dominant la recherche. Cette représentation consistait à identifier ces familles comme des milieux, sinon complètement pathologiques, du moins suffisamment dysfonctionnels pour susciter une distanciation, quand ce n'était pas le mépris. "Les parents d'enfants retardés mentaux sont confus, sur la défensive et irrités. Des souhaits de mort apparaissent dans la majorité des interviews. C'est pourquoi les parents tentent de résoudre leurs problèmes émotionnels en faisant preuve d'une sollicitude excessive envers leur enfant, par la surprotection et une trop grande indulgence à son égard. » Cette phrase de Smith (1952) illustrait un discours encore présent dans la plupart des pays européens vers 1970.

Confrontés sans cesse à des familles, tout d'abord dans les services d'aide précoce nouvellement créés, puis progressivement durant la période de scolarité des enfants, nous avons commencé une véritable formation sur le terrain, participant à l'évolution des idées dans ce domaine.

\section{L'ÉVOLUTION DES PERSPECTIVES}

Les connaissances relatives aux familles d'enfants déficients intellectuels ont subi des transformations majeures au cours des trois dernières décennies et plus particulièrement dans le domaine de l'adaptation familiale. 
C'est en effet le domaine ayant recueilli les suffrages des chercheurs, lesquels ont développé différentes trames théoriques de plus en plus complexes pour rendre compte des phénomènes de stress et de coping. Comme nous allons le montrer, les interrogations du départ centrées exclusivement sur la souffrance parentale ont laissé place à l'étude des processus utilisés par les parents pour s'adapter à la présence de leur enfant et l'intégrer dans leur développement familial.

\section{LA DÉTRESSE CHRONIQUE}

Les premiers travaux utilisèrent un modèle emprunté à la psychologie clinique, laquelle reposait sur un postulat précis, l'existence d'une norme définissant la famille "saine " (Farber, 1960). Les recherches étaient centrées sur la définition de deux grandes catégories de variables. La première englobait les facteurs indiquant les écarts par rapport à la norme. Les thèmes privilégiés furent les conflits, les tensions, les troubles de la personnalité, les inhibitions parentales et les difficultés exprimées par les fratries. La seconde catégorie regroupait les besoins en services destinés à améliorer la vie des parents.

En Europe de langue française, cette perspective trouva dans la psychanalyse un lieu de prédilection. Les réactions parentales furent décrites et classées comme angoisse, déni, avec un accent particulier placé sur les mécanismes de défense comme la rationalisation et la substitution. Par exemple, dans une étude consacrée aux réactions de parents lorsqu'on leur a annoncé que leurs enfants étaient atteints de trisomie 21, certaines déclarations parentales étaient cataloguées comme des "refus de connaître la vérité", des "réponses intellectuelles peu adaptées", voire des "signes d'agressivité » (Lambert, 1978). Dans les faits, la souffrance vécue par les parents était en quelque sorte " théorisée "; elle acquérait ainsi un caractère chronique se traduisant par des perturbations psychologiques individuelles affectant tous les membres d'une même famille. Notons qu'une approche des réactions parentales au stress comme mécanismes de défense est intégrée dans les théories actuelles du coping et plus particulièrement dans la conception offerte par Lazarus et Folkman (1984). La différence essentielle se situe dans le statut accordé à ces mécanismes. Pour la psychanalyse, il s'agit de processus largement inconscients qu'il convient d'identifier et de faire découvrir aux personnes touchées. Dans les théories du coping, on part de l'idée que les personnes veulent connaître ce qui leur arrive, ce que cela signifie pour leur bienêtre et qu'elles préfèrent porter un regard positif sur les choses. Toute appréciation est le produit d'interactions entre l'environnement et ce que 
souhaite la personne. En postulant que les réponses à une situation de stress sont médiatisées par des processus cognitifs, les théories du coping suggèrent que les appréciations des individus ne sont pas les seuls produits de leurs motivations et de leurs mécanismes de défense, mais que ces jugements personnels agissent avec le milieu.

Avec la psychanalyse, nous restions cependant en présence d'une approche de type psychopathologique. La famille avec un enfant déficient intellectuel était abordée sous l'angle de ses réactions à une situation de stress, celle-ci devant être considérée comme induite a priori par la déficience. Ce modèle ne résista pas longtemps à deux observations centrales. La première venait des ouvrages scientifiques et plus particulièrement des travaux de chercheurs anglais qui relevaient çà et là des phénomènes adaptatifs intéressants, certaines familles continuant à vivre " normalement " longtemps après la naissance de leur fils ou de leur fille déficient (Carr, 1974). La seconde observation était issue de la clinique, des entretiens avec les familles. Nous constations, encore confusément à l'époque, que nous n'étions pas en présence de personnalités troublées psychiquement, de pathologies mentales bien ancrées, celles-ci étant en fait des exceptions. Surgirent alors d'autres questions. Qu'est-ce que l'ajustement familial? Quelles sont les variables à prendre en considération? $\mathrm{Qu}^{\prime}$ est-ce qu'une famille normale?

En fait, ce modèle psychopathologique individuel centré sur les désorganisations affectant chaque membre d'une famille comportait une lacune au départ de son raisonnement, $\mathrm{c}^{\prime}$ est-à-dire l'absence d'une définition de la famille " normale ». En outre, il réduisait des signes de dysfonctionnement à une problématique individuelle, négligeant ainsi de prendre en compte la famille comme un système complexe, à la fois dans ses interactions internes et dans ses relations avec les différents niveaux de l'organisation sociale.

Deux éléments doivent cependant être portés au crédit de cette approche. Elle fut la première à envisager la famille sous l'angle de ses réponses à une situation de stress, mettant en avant les quatre composantes qui allaient servir de base aux travaux ultérieurs: l'événement générateur de stress, les ressources, l'interprétation de la situation et la crise. Ensuite, cette perspective fut à l'origine de la création des premiers services destinés aux familles. On vit apparaître des structures d'aide aux familles, prenant des formes diverses allant du conseil ambulatoire à la formation de parents à l'intérieur de séminaires structurés, ceux-ci étant notamment influencés par les principes de l'analyse comportementale (Lambert, 1980). 
Les vues réductionnistes du modèle clinique ne doivent toutefois pas faire perdre de vue une réalité. De nombreuses familles souffrent incontestablement de la présence d'un enfant déficient intellectuel. Cette souffrance s'exprime de manières diverses. Elle n'est pas nécessairement chronique, comme le pensent les théoriciens de ce mouvement. Elle est néanmoins présente et ne peut en aucun cas être évacuée par les chercheurs et les professionnels sous prétexte de l'inadéquation d'un modèle théorique. C'est cette souffrance que tentèrent de mieux apprécier les travaux qui suivirent en proposant des modèles permettant d'expliquer le stress vécu par les familles.

\section{LE STRESS ET LES RESSOURCES}

\section{L'ÉVALUATION DU STRESS : ANALYSE CRITIQUE}

Dès le début des recherches, nous avons été confrontés au manque d'instruments standardisés permettant de mettre en lumière les facteurs de stress et de ressources dans les familles. En 1991, nous disposions d'un modèle du fonctionnement familial apparemment efficace (Lambert et Lambert-Boite, 1993). Nous savions que l'efficacité des ressources mises en œuvre par la famille pour résoudre une crise dépendait de la manière dont les parents définissent et interprètent les besoins de l'enfant, son niveau de fonctionnement et ses problèmes comportementaux. Un fait s'imposait: un problème chez l'enfant ne provoque pas nécessairement un stress dans la famille. Certaines familles étaient capables d'aborder une difficulté de manière positive et de l'inclure dans leur routine quotidienne. Pour d'autres, cela constituait un obstacle quasi insurmontable, une source de déséquilibre. Nous utilisions cependant le concept de stress comme une donnée acquise. Un article au titre très suggestif a permis de franchir une nouvelle étape dans la compréhension des phénomènes habituellement regroupés sous le concept de stress: "Ce que nous ne savons pas au sujet des familles ayant un enfant retardé " (Glidden, 1993).

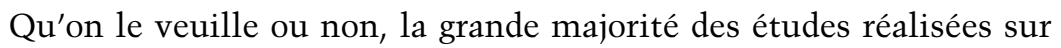
le stress se fonde implicitement sur le modèle clinique suivant lequel la présence d'une personne retardée dans le milieu familial se voit attribuer une causalité pathologique d'adaptation familiale. Les instruments mis en place pour mesurer cette influence conceptualisent en conséquence la pathologie en fonction du stress. Il n'est guère surprenant de constater que les familles ayant un enfant déficient présentent des degrés de stress supérieurs aux autres milieux. Cette découverte a été utilisée pour confirmer l'hypothèse de départ selon laquelle ces familles présentent généralement des troubles d'adaptation. Une acceptation inconditionnelle de 
cette perspective ne survit pas à l'épreuve de la réalité. Les résultats disponibles sont nettement contradictoires et ne permettent pas de dégager des lignes précises pour l'intervention. Deux raisons expliquent cette situation. D'une part, l'adaptation familiale n'est pas une question de tout ou de rien liée à l'absence ou à la présence de stress. Les chercheurs négligent en quelque sorte les ressources dans le modèle, en omettant le rôle joué par les forces individuelles et familiales dans l'adaptation. Parmi ces forces, citons les capacités de résolution de problèmes, l'auto-évaluation positive, le degré de scolarisation, les possibilités financières, le degré de satisfaction conjugale, la cohésion familiale, les croyances parentales et le réseau de soutiens sociaux formels et informels (Lambert et LambertBoite, 1993). D'autre part, l'utilisation du concept même de stress est ambiguë dans les ouvrages sur la déficience intellectuelle, tout se passant comme si les chercheurs n'étaient pas au courant des développements théoriques importants qu'a connus cette notion (Lazarus et Folkman, 1984). L'observation sur le terrain permet d'illustrer nos propos.

Prenons l'exemple d'une famille dans laquelle un enfant déficient a besoin de quatre séances hebdomadaires de physiothérapie pour réduire les séquelles d'une atteinte motrice. La manière dont la famille répond à une telle exigence dépend de nombreux facteurs, parmi lesquels les ressources financières, la situation géographique, la disponibilité respective des parents et la présence d'un service spécialisé jouent un rôle incontestable. Il est très possible que cette famille ne considère pas cette exigence comme un stress si des conditions personnelles et sociales lui permettent d'affronter la demande. Il en ira tout différemment dans une autre famille confrontée à une situation analogue mais ne bénéficiant pas, par exemple, de l'apport d'un service adéquat dans son entourage. En d'autres termes, un événement donné prend une signification spécifique en fonction de l'écologie familiale.

Lazarus et Folkman (1984) proposent une théorie du coping soulignant l'importance des significations apportées à une situation de stress. Pour ces auteurs, l'élément clé est l'appréciation de l'événement stressant. Cette appréciation dépend de facteurs personnels (préférences, croyances) et situationnels (nouveauté, prévisibilité, soudaineté, durée, intensité). Une première évaluation cognitive consiste à analyser et à qualifier l'événement comme peu important, favorable ou stressant. Cette appréciation primaire est suivie d'une analyse des stratégies de résolution et des ressources disponibles. Durant cette appréciation secondaire, la personne dresse un plan d'action et estime ses chances de réussite. Ces stratégies et ces ressources dépendent de facteurs psychologiques et environnementaux, des forces individuelles et familiales. Si de nouvelles informations 
surgissent durant cette évaluation cognitive, un processus de réappréciation est mis en action. Si la personne juge que l'événement stressant nécessite des ressources dépassant les siennes, elle entreprend un processus de coping, c'est-à-dire un ensemble d'efforts cognitifs et comportementaux visant à répondre aux demandes externes ou internes face auxquelles elle s'est d'abord sentie démunie. L'évaluation cognitive de l'événement est donc centrale dans cette approche du coping.

D'une manière générale, les questionnaires utilisés en déficience intellectuelle pour évaluer le stress confondent les demandes et le stress. Les demandes sont évidemment plus importantes dans les familles des personnes déficientes, mais elles ne se traduisent pas nécessairement en stress.

Prenons l'item suivant: “Mon enfant a des comportements inhabituels qui attirent l'attention à l'extérieur ", auquel de nombreuses familles répondent "vrai” dans le questionnaire. Cette particularité comportementale qu'ont de nombreux enfants déficients est indéniablement un stresseur potentiel. Elle n'acquiert le statut de stress que si les parents sont dérangés par ce comportement et ne peuvent pas supporter l'attention que des personnes extérieures portent à leur enfant. Un autre item stipule: “Je souhaiterais que mon enfant soit normal. "Nous ne connaissons pas de parents d'enfant déficient intellectuel pouvant répondre "faux" à une telle affirmation. Nous ajouterons que toute comparaison entre familles avec personne déficiente intellectuelle et familles sans personne déficiente intellectuelle est suspecte parce que les questionnaires ont été prévus pour les familles de personnes déficientes, certains items ayant une probabilité d'apparition quasi nulle dans les autres. Les chercheurs continuent néanmoins à prétendre que l'obtention de scores élevés aux instruments mesurant le stress est prédictive de l'inadaptation.

Force est de reconnaître que l'absence de normes, la faiblesse méthodologique des instruments et l'escamotage théorique limitent singulièrement l'utilisation des instruments disponibles et obligent la recherche à proposer d'autres voies pour la pratique (Clayton, Glidden et Kiphart, 1994). D'une part, il y a lieu d'introduire une distinction entre la fréquence du stress et son intensité ressentie dans les instruments destinés aux parents d'enfants déficients. En d'autres termes, il convient de mettre l'accent sur les appréciations cognitives des familles face à un événement. D'autre part, les différences individuelles sont importantes. Chaque famille réagit à la présence d'un enfant déficient en fonction de ses perceptions cognitives, de ses attentes, de ses ressources internes et 
externes. Cela rend certes difficile la construction de normes, d'outils standardisés, mais c'est la seule issue qui s'offre aux chercheurs pour tenter de dépasser les confusions actuelles.

\section{DES ÉLÉMENTS EN INTERACTION}

Aujourd'hui encore, les travaux sur le stress des familles d'enfants déficients intellectuels représentent la contribution majeure des chercheurs dans le domaine de l'adaptation familiale. D'emblée, les chercheurs utilisèrent des modèles du stress familial développés dans les années d'aprèsguerre (Hill, 1949). Notre propos n'est pas une présentation exhaustive des différents modèles mis au point pour tenter d'expliquer le stress dans les familles d'enfants déficients intellectuels. Le lecteur intéressé consultera avec profit deux excellentes synthèses lui permettant d'évaluer le chemin parcouru en une décennie (Minnes, 1988; Baker, Blacher, Kopp, et Kraemer, 1997). À l'aide d'un exemple, nous illustrons les modifications survenues dans l'étude de ce phénomène.

D'une manière générale, l'approche du stress s'est basée sur un modèle prenant en compte quatre éléments symbolisés par des lettres: A : l'événement stressant; B: les ressources familiales; C: la définition faite par la famille de l'événement stressant; et X: la crise, le stress. On considérait que ces éléments survenaient de manière linéaire, A entraînant $B$ et ainsi de suite. Les chercheurs utilisent deux grands modèles explicatifs, appelés respectivement ABCX et ACBX.

Joanne, fillette autiste âgée de cinq ans, est intégrée au mois de septembre dans un jardin d'enfants de son quartier. Les premières semaines se passent sans grandes difficultés. Cette intégration se réalisant à mi-temps, Joanne fréquente le reste de la semaine un centre spécialisé où elle reçoit l'éducation nécessaire à ses problèmes de développement, caractérisés notamment par une expression verbale difficile et des difficultés de participation à la vie en groupe, difficultés se traduisant épisodiquement par des réactions d'agressivité et des périodes d'apathie. Observant les progrès de sa fille, la maman, divorcée, demande que Joanne soit davantage intégrée dans le jardin d'enfants et qu'elle puisse bénéficier en quelque sorte d'un plan d'intervention “à la carte ", consistant notamment en heures d'intervention spécialisée dans le jardin d'enfants. Cette solution permet également à la mère d'éviter des déplacements peu compatibles avec son activité professionnelle, l'école du quartier et le centre spécialisé étant distants d'une dizaine de 
kilomètres. Après concertation, l'administration du jardin d'enfants et celle du centre spécialisé refusent cette solution et préconisent le partage hebdomadaire de Joanne entre ces deux milieux. La mère ne peut plus faire face à la surcharge qu'imposent les déplacements de la fillette. Seule à la maison, elle doit en outre s'occuper d'un fils de huit ans qui fréquente l'école primaire et demande son attention.

Dans le modèle du stress présenté ci-dessus, le refus des instances administratives est considéré comme l'événement stressant (A). Les ressources de la mère sont en fait peu nombreuses, son activité professionnelle, la charge des déplacements et son fils aîné lui permettant difficilement de poursuivre l'expérience d'une intégration à mi-temps (B). L'appréciation faite par la mère de l'événement stressant est manifestement forte: elle vit mal cette situation de surcharge familiale. Elle reconnaît cependant que le degré de développement de Joanne est suffisamment bon pour tenter une expérience d'intégration à mi-temps, une situation qui n'a pas été possible pour trois autres enfants du groupe de parents $\mathrm{d}^{\prime}$ enfants autistes dont elle est membre $(\mathrm{C})$. C'est toutefois le refus administratif qui a eu l'influence la plus grande, la mère ayant " craqué nerveusement ", selon son expression, et ayant dû être prise en charge par un psychologue $(\mathrm{X})$. Par la suite, elle a toutefois pris une décision pour Joanne: lui laisser terminer l'année en intégration partielle et refaire la demande aux autorités au début de la prochaine année scolaire.

Ce modèle, popularisé dans les ouvrages sous le sigle $\mathrm{ABCX}$, a subi diverses transformations. McCubbin et Patterson (1983) l'ont élargi en parlant d'un modèle “ABCX double », indiquant par là qu'une crise peut mener à une accumulation de facteurs de stress qui conduit la famille à redéfinir l'événement stressant, à réévaluer les ressources, à en rechercher d'autres et à réinterpréter la nouvelle situation. $\mathrm{Si}$, au terme de cette nouvelle appréciation, les ressources font encore défaut, la crise se déclenche.

Un tel modèle reste cependant très statique. En effet, il considère la suite des événements se passant dans le milieu familial sous un angle linéaire, négligeant ainsi les nombreuses interactions que peuvent avoir les éléments entre eux et plus particulièrement l'importance de la perception qu'ont les parents des événements qui les touchent. Orr, Cameron et Day (1991) apportèrent un éclairage nouveau à cette perspective dans une étude sur la sévérité des problèmes comportementaux d'enfants déficients intellectuels sévères, ces troubles du comportement étant considérés comme des événements stressants (A). L'originalité de l'approche se situe dans les relations entre la définition faite par la famille de l'événement 
stressant $(\mathrm{C})$ et la crise $(\mathrm{X})$ : cette perception n'est pas directement liée à l'expérience du stress. En d'autres termes, l'utilisation des ressources dépend plus de la perception qu'ont les membres de la famille de la gravité des problèmes comportementaux que de la sévérité réelle de ces derniers. Le modèle est devenu ACBX. Il peut paraître dérisoire d'attribuer des lettres à des situations affectives et sociales, et de réduire le stress en une équation. Cette approche a cependant contribué à transformer progressivement notre manière d'appréhender la réalité des familles (Lambert, Piret, Scohy et Lalière, 1993).

Le mérite du modèle ACBX, renforcé par une recherche de Willoughby et Glidden (1995), est de susciter la réflexion sur les concepts euxmêmes. En fait, l'efficacité des ressources mises en œuvre par la famille pour résoudre une crise dépend de la manière dont les parents définissent et interprètent les besoins de l'enfant, son niveau de fonctionnement et ses problèmes comportementaux. On ne peut pas prédire qu'un problème chez l'enfant va nécessairement entraîner un stress dans la famille. Certaines sont capables d'aborder une difficulté de manière positive et de l'inclure dans leur routine quotidienne. Pour d'autres, cela va constituer un obstacle quasi insurmontable et entraîner des déséquilibres. Une telle perspective indique aux praticiens que toute intervention doit d'abord analyser de façon précise la manière dont les parents perçoivent leur enfant, puis déterminer dans quelle mesure cela affecte la famille. Dans l'exemple de Joanne, il aurait été nécessaire de prendre en considération dès le départ l'interprétation que la mère donnait au refus, c'est-à-dire la surcharge domestique causée par les déplacements et son souci de préserver le développement de son fils aîné.

Il existe d'autres modèles du stress familial utilisés en déficience intellectuelle. Ces travaux ont eu deux impacts évidents dont nous mesurons encore aujourd'hui l'importance. D'une part, l'atout principal des études a été de mettre l'accent sur les ressources comme des éléments essentiels pouvant contrecarrer les facteurs de stress. D'autre part, les recherches ont fait ressortir un élément essentiel dans l'abord de l'adaptation familiale, c'est-à-dire la description des variables qui influencent l'appréciation de la situation, variables parmi lesquelles les émotions jouent un rôle central.

En reprenant l'exemple de Joanne, le chercheur se pose la question suivante: Quels sont les facteurs qui font que la mère interprète la situation comme stressante? De nombreux éléments doivent être pris en considération: le fait d'être seule pour éduquer deux enfants, la présence de sa fille autiste âgée 
de cinq ans qui nécessite un engagement éducatif important, les contraintes matérielles et financières imposées par les déplacements, les demandes légitimes du fils aîné, les représentations maternelles sur l'intégration d'un enfant déficient et les aspirations à voir se développer une scolarité régulière, la qualité des contacts avec le père des enfants, l'étendue et la qualité du réseau social développé ou conservé par la mère, les décisions des responsables administratifs qui se retranchent derrière les règlements, etc. Ces éléments n'agissent certainement pas de manière isolée, ils sont en interaction. En outre, ils appartiennent à des sphères très diverses du fonctionnement individuel et familial. Comment peut-on mettre en lumière l'importance de chaque variable? Quel est le rôle des réactions émotionnelles dans le processus de décision? Quels sont les moyens dont dispose la mère pour affronter les composantes de sa situation?

Progressivement, semblables interrogations ont amené les chercheurs à se rendre compte que ces facteurs étaient complexes, multidéterminés, qu'ils touchaient tout autant des constellations de variables personnelles, que des éléments extérieurs à la famille. Le modèle ACBX se révèle une référence utile pour aborder le stress et le coping dans les familles d'enfants déficients. Toutefois, pour apprécier l'ensemble de la situation, il manquait une perspective plus générale prenant en compte à la fois la diversité des variables personnelles et familiales et l'ensemble des milieux dans lesquels évoluent les familles d'enfants déficients. La perspective écologique s'impose comme le nouveau cadre permettant d'envisager l'ensemble des facteurs intervenant dans l'appréciation des événements survenant dans la vie des familles d'enfants déficients.

\section{UNE PERSPECTIVE ÉCOLOGIQUE}

$\mathrm{Au}$ cours de la dernière décennie, une nouvelle approche conceptuelle s'est dégagée dans le monde de la déficience intellectuelle. Il s'agit du modèle organisationnel que l'on nomme également socioécologique. Il intègre les différentes dimensions des connaissances actuelles, notamment en analysant les ressemblances et les spécificités qu'entretiennent les milieux familiaux des enfants déficients intellectuels par rapport aux autres familles. En fait, ce type de raisonnement repose sur une évidence. Il a fallu longtemps en effet pour que les chercheurs prennent en compte une réalité qui s'imposait à toute personne travaillant avec des familles d'enfants déficients, à savoir que ces familles sont constituées de personnes 
qui s'organisent, fonctionnent, vivent, interagissent et font face avec plus ou moins de bonheur aux réalités quotidiennes. Cette étape rompt définitivement avec l'équation " une famille avec un enfant déficient est une famille pathologique ".

L'adoption de ce cadre socioécologique dans le monde de la déficience $\mathrm{n}^{\prime}$ est somme toute que la transposition dans cet univers d'une perspective ancrée depuis une vingtaine d'années dans l'éducation familiale générale. Quatre éléments centraux constituent les bases du modèle.

\section{LA FAMILLE EST UN ÉCOSYSTĖME}

Le modèle propose une articulation des faits et des thèmes de recherche en considérant la famille comme un écosystème éducatif. Cette position s'inspire des travaux de Bronfenbrenner $(1977 ; 1979)$. La théorie distingue cinq sous-systèmes.

Laurent, atteint de trisomie 21 et âgé de 12 ans, est le troisième enfant d'une famille. Il a deux sœurs âgées de 14 et 18 ans. Les parents travaillent tous les deux, le père comme monteur électricien, la mère comme institutrice maternelle à temps partiel. Ils habitent dans un appartement d'une petite ville dans laquelle on trouve, en matière de services, une école spécialisée et un centre d'intervention psychologique avec plusieurs collaborateurs. Laurent est en dernière année de scolarité primaire dans une classe spéciale. Nous avons l'occasion de le rencontrer à la demande conjointe des parents et de l'instituteur. Depuis deux mois, Laurent ne participe plus aux activités scolaires. Il a perdu son intérêt pour les choses qu'il aimait faire, en particulier assister à des rencontres de basket-ball. Il se plaint de maux divers. Il veut rester à la maison et déclare quotidiennement ne plus souhaiter se rendre à l'école. Tous les examens médicaux ont donné des résultats négatifs. Les parents s'inquiètent de son évolution. Lorsque nous les rencontrons pour la première fois, le père nous dit que l'usine où il travaille va peut-être fermer ses portes. Le désarroi des parents est d'autant plus important que le développement de Laurent n'avait guère posé de problèmes jusque-là. La vie à la maison et à l'école devient problématique. La mère nous déclare que sa fille de 14 ans supporte mal la situation et qu'elle marque son malaise par des crises d'opposition. Normalement, Laurent 
devrait quitter l'école spéciale au terme de l'année en cours pour fréquenter un établissement pour adolescents situé dans une ville proche de quinze kilomètres.

L'application du modèle écologique à cette situation propose de distinguer les différents éléments selon leur appartenance à un sous-système.

Il y a tout d'abord le milieu immédiat, la maison, l'école, le voisinage, au sein duquel les acteurs s'engagent dans des activités (microsystème). Cela inclut l'environnement physique. Toutes les personnes de la famille sont impliquées dans des rôles (endosystème). Ces rôles s'organisent en styles. Par exemple, on analyse les rôles du père et de la mère, les conditions de fonctionnement de la fratrie, le degré de satisfaction conjugale. L'enfant participe à différents milieux (mésosystème). Cela comprend les relations avec la famille, l'école, le groupe d'âge. Ces relations sont influencées par des structures sociales concrètes (exosystème): les institutions sociales qui gèrent la distribution des biens et des services ou les classes sociales en tant que facteurs d'influence. Enfin, il y a des structures globales à l'échelle régionale ou nationale (macrosystème): systèmes juridiques, éducatifs, politiques.

Ce cadre de référence permet d'apprécier la situation de Laurent et de sa famille en ciblant les grandes influences à chaque niveau de l'écologie. Le problème central qui se pose avec ce modèle est simple à formuler, mais difficile à résoudre (Lambert, 1995). Cette approche ne nous renseigne pas en effet sur les critères à utiliser pour ordonner les composantes écologiques selon leurs effets sur le fonctionnement familial. Pour Laurent et sa famille, est-il plus important d'aborder la constellation familiale en tant que ressource dans le milieu immédiat ou de privilégier la piste des contacts entre la famille et l'école? Faut-il inclure le père ou les sœurs dans un processus de modification des interactions? Quel est le rôle joué par la fin proche de la scolarité dans une petite école où Laurent a passé six ans? Quelle est l'importance de la perte éventuelle de l'emploi paternel dans la situation?

Pour aborder ces questions, qui posent le problème de la hiérarchisation des éléments formant le fonctionnement familial, il est nécessaire de se pencher sur les contextes d'activité des personnes (Nihira, Weisner, et Bernheimer, 1994). Les routines quotidiennes dans lesquelles s'engagent les membres d'une famille constituent l'architecture de leur fonctionnement. Les familles ne sont pas les victimes quasi obligées de forces sociales et économiques. Dans la réalité, les familles développent des actions collectives afin d'agir contre ces forces et de les modifier. C'est finalement l'analyse de ce qu'on appelle la niche écologique qui fournit 
des hypothèses de travail. La niche familiale reflète l'écologie matérielle telle qu'elle est définie de manière traditionnelle: le revenu, les conditions de santé, la maison, l'espace de vie, les transports, etc. Elle comprend également les facteurs culturels utilisés par les personnes pour comprendre et organiser leur vie quotidienne: les croyances et les objectifs moraux, les origines et les causes de la déficience, les conduites culturelles liées au couple et aux relations familiales. La niche écologique n'est pas une entité statique. Elle se modifie en fonction de l'adaptation, c'est-à-dire des stratégies utilisées par la famille pour s'adapter, exploiter, contrebalancer et réagir aux forces en présence. Au fur et à mesure que les parents décrivent leurs conduites, analysent leurs stratégies, prennent en considération les solutions possibles et aménagent une routine stable, ils révèlent comment ils construisent le cadre de vie de leur famille.

\section{LA PERSPECTIVE TEMPORELLE EST IMPORTANTE}

Le fonctionnement familial doit être considéré sur un double axe temporel: le quotidien et le longitudinal. L'étude de l'évolution d'une famille à un moment précis de son existence néglige des données importantes. Les données des recherches sont suffisamment nombreuses pour montrer que les dimensions de l'adaptation familiale varient selon le temps. Parmi les plus importantes, citons: les besoins des parents qui différent selon l'âge des enfants, l'évolution des perceptions de la déficience par les parents, la recherche de réseaux de soutien social, le devenir des fratries, l'impact des troubles du comportement de l'enfant déficient sur le fonctionnement individuel des parents (Lambert et Lambert-Boite, 1993).

Les deux axes temporels, le quotidien et le longitudinal, doivent être pris en considération car ils ne se recoupent pas. En effet, le premier agit de manière accessoire. Cela ressort des interviews des parents. De nombreuses familles passent quotidiennement par différents états, du découragement temporaire à l'élaboration de sentiments positifs d'efficacité. La vie familiale est ainsi faite. La triste mine de Laurent au repas du matin qui, après une nuit difficile, ne répond pas aux sollicitations et refuse de manger, sera mal reçue par les parents au départ d'une journée de travail. En fin de journée, le fait que Laurent entre de l'école avec un cahier et des annotations positives de la part de l'enseignant en racontant qu'il a participé à un match de football avec ses pairs, modifie le tableau. La mère et le père puisent dans cette indication une source d'espoir, certains 
d'une amélioration de l'état de leur enfant, convaincus que le fait de continuer à l'envoyer à l'école est une bonne solution. L'ambiance familiale au repas du soir n'aura plus aucun rapport avec celle du matin.

Le second axe temporel induit également des modifications, certes moins spectaculaires, mais aux effets plus permanents. La prise en considération de cette perspective s'impose à la fois pour apprécier l'évolution des processus adaptatifs et pour mettre en place des programmes éducatifs efficaces. Pour Laurent et sa famille, il est nécessaire de tenir compte du moyen terme, c'est-à-dire la fin de la scolarité dans la petite école où il a tous ses compagnons et compagnes, avec un enseignant qui l'a suivi depuis trois ans. Il s'agit d'une classe spéciale, non intégrée, comme la majorité des classes de ce type en Suisse, accueillant six élèves tous déficients intellectuels. En fait, Laurent est dans une phase de transition. À nouveau, les résultats de plusieurs recherches montrent combien il est nécessaire de prévoir les transitions chez les enfants déficients intellectuels parce qu'elles modifient les familles, parfois de manière radicale (Lambert et Lambert-Boite, 1993). Pour Laurent, c'est l'entrée dans l'adolescence et le changement futur d'école qui sont deux réalités. Cette transition doit être préparée avec la famille et l'enfant, en envisageant toutes les implications pour le fonctionnement familial.

\section{LE STRESS ET LES RESSOURCES SONT EN INTERACTION}

Le modèle organisationnel a intégré les travaux sur le stress et les ressources présentés ci-dessus en accentuant leurs interactions et le rôle important joué par l'analyse que font les parents de leur situation. L'originalité de la démarche est d'introduire une recherche des facteurs de stress et de ressources à tous les niveaux de l'écosystème familial.

L'observation du milieu et la récolte de données tangibles sur les facteurs de la tension éprouvée par les parents et leurs ressources s'imposent aujourd'hui comme des préalables à toute démarche éducative et doivent précéder l'évaluation systématique du degré de développement de l'enfant au moyen d'instruments standardisés.

\section{L'ADAPTATION FAMILIALE EST UN PROCESSUS}

Dernière composante du modèle organisationnel, l'appréciation de l'adaptation familiale est en quelque sorte l'aboutissement de la démarche d'analyse. Opérationnellement, la définition suivante de l'adaptation peut être considérée comme suffisante: il s'agit des réponses apportées par les milieux familiaux aux problèmes quotidiens posés par la présence d'un 
enfant déficient intellectuel, ces problèmes touchant aussi bien les domaines affectif et cognitif que la sphère matérielle et le champ des projections et des aspirations.

L'adaptation familiale ne peut pas toutefois s'apprécier dans le vide, mais en relation avec l'environnement physique et social quotidien présenté à l'enfant déficient. L'originalité de la démarche actuelle en déficience intellectuelle consiste dans la mise en lumière des interactions entre le stress, les ressources et la qualité des stimulations physiques et sociales assurées par les familles. Il reste cependant que nous manquons encore actuellement de données permettant de comprendre les apports réciproques de la famille et de l'enfant déficient dans le processus adaptatif. Une piste récente indique que l'âge de l'enfant et certaines caractéristiques comme son niveau intellectuel et les troubles du comportement adaptatif modèlent significativement les réactions adaptatives des parents (Keogh, Garnier, Bernheimer et Gallimore, 2000). Cette recherche porte cependant sur des enfants qui présentent des retards de développement avec des étiologies non identifiées, et ses résultats ne peuvent donc pas être généralisés aux déficiences intellectuelles d'origine génétique.

\section{LES DEMANDES DES PARENTS ET LE PARTENARIAT}

\section{DES DEMANDES MULTIPLES}

L'étude des familles d'enfants déficients intellectuels s'est enrichie récemment d'un dernier courant mettant l'accent sur les besoins. Les familles ont des besoins. Il est surprenant de constater que cette réalité n'a guère retenu l'attention des chercheurs avant la publication d'un article déterminant (Bailey et Simeonsson, 1988). Nous dispenserons le lecteur d'une analyse théorique fastidieuse de la notion de besoin, un concept utilisé en psychologie à tort et à travers. Pour éviter toute ambiguitté, nous lui préférons le terme "demande ". C'est au terme d'un ensemble de recherches sur l'adaptation des familles de jeunes enfants déficients que Bouchard, Pelchat, et Boudreault (1996) ont mis en lumière l'importance des demandes familiales pour imposer cette perspective dans les travaux de langue française.

À partir des travaux de Bailey et Simeonsson (1988), nous avons traduit et adapté le Family Need Survey, sous la forme d'une échelle regroupant 35 demandes de base dont les qualités psychométriques ont été mises à l'épreuve avec plusieurs centaines de parents (Bailey, Blasco et Simeonsson 1992). La synthèse des données effectuée par Simeonsson et Bailey (1993) et portant sur trois études dûment contrôlées est toujours d'actualité. En outre, les demandes formulées par des parents d'enfants 
en âge scolaire sont actuellement bien connues (Westling, 1996). Toutes ces recherches ont été menées aux États-Unis. Il nous est apparu nécessaire de les transposer dans la francophonie européenne et nord-américaine afin de les adapter à nos réalités culturelles. Différentes études menées dans notre centre ont apporté des transformations dans l'instrument initial. Nous avons conservé 32 demandes. Nous disposons actuellement des résultats obtenus auprès de 286 parents. Les qualités métriques de l'instrument et les différentes analyses factorielles sont présentées ailleurs (Lanners, 1997; Lanners et Lambert, 1999).

Quatre grandes catégories de demandes se dégagent, quel que soit l'âge de l'enfant.

- les demandes en informations recouvrent des domaines divers: sur la manière de parler et de jouer avec l'enfant, son développement, les comportements, l'apprentissage, les services disponibles, l'enfant, son retard, ses difficultés;

- les demandes de soutien se classent en trois groupes:

- les soutiens formels professionnels: rencontrer plus fréquemment un conseiller, aide pour comprendre et accepter l'enfant, pour discuter en famille et trouver des solutions, aide pour apprendre à s'aider mutuellement et pour répartir les tâches familiales;

- les soutiens formels non professionnels: associations de parents, recherche de gardiennes, accès aux écrits d'autres parents;

- les soutiens informels: avoir des amis ou un membre de la famille pour parler, disposer de temps pour soi;

- les demandes de moyens financiers liées surtout à un accroissement des dépenses occasionnées par l'enfant, ses traitements, l'achat de matériel adapté;

- les demandes liées à l'intégration sociale de l'enfant:

- les demandes d'aide pour parler de l'enfant déficient intellectuel à différentes personnes: fratrie, d'autres enfants, le conjoint et les grands-parents, les amis, les voisins et l'enseignant de la classe spécialisée;

- les demandes de services adaptés: un centre de jour ou une école.

Nous avons analysé ces demandes à partir de plusieurs variables démographiques (âge de l'enfant déficient intellectuel, sexe, ordre des naissances; pères et mères) et sociales (niveau socioculturel, localisation géographique des familles, proximité de services). Trois données importantes pour l'étude des familles s'imposent. 
Premièrement, les pères n'expriment pas moins de demandes que les mères. L'âge des parents n'exerce aucune influence sur le nombre total de demandes exprimées. D'une manière générale, les demandes des pères et des mères sont identiques. Les seules différences enregistrées entre eux se situent dans les soutiens. Les mères expriment significativement plus de demandes en soutien formel non professionnel. Cela correspond aux données de l'étude américaine et aux résultats des travaux commençant à cerner le rôle des pères dans les familles d'enfants déficients intellectuels (Lillie, 1993; Willoughby et Glidden, 1995). D'une manière générale, les demandes des pères à l'égard des services sont réduites lorsque les enfants sont jeunes, les habitudes culturelles mettant encore souvent la mère au centre des conduites éducatives et domestiques durant les premières années. Les mères ressentent plus le besoin de bénéficier de support formel et informel durant ces années parce qu'elles endossent plus de responsabilités dans l'éducation. Par la suite, dès l'entrée à l'école, les pères expriment les mêmes demandes que les mères à l'égard des services (Lambert et Studer, 1998).

Deuxièmement, le sexe de l'enfant déficient intellectuel influence la formulation de demandes par les parents. Les familles de garçons expriment significativement plus de demandes en information dans trois domaines: les services disponibles, l'enfant et son retard, et la manière de s'y prendre avec les troubles du comportement. Deux hypothèses sont plausibles. La première est d'ordre culturel. Les attentes envers un garçon restent plus importantes dans les milieux étudiés. Le fait que le garçon soit déficient intellectuel accroît l'attente envers les capacités de l'enfant, en même temps que la souffrance d'avoir donné naissance à un être déficient (Beckman 1983; 1991). Sa seconde hypothèse tiendrait compte d'une fréquence plus grande des difficultés comportementales chez les garçons, un phénomène expliquant en partie une plus grande recherche d'information chez les parents concernés. Les travaux épidémiologiques récents ne permettent toutefois pas de confirmer cette tendance (Einfeld et Tonge, 1996).

Troisièmement, l'âge de l'enfant est une variable également significative dans l'expression des demandes, celles-ci diminuant avec l'âge. Les parents d'un enfant âgé de moins d'un an expriment significativement plus de demandes. Par contre, il n'y a pas d'interactions significatives entre l'âge et les types de besoins. Ce résultat n'est pas une surprise. Il s'agit seulement d'une réduction quantitative, l'âge n'exerçant aucune influence sur une évolution qualitative des catégories de demandes. On imagine aisément que plus l'enfant grandit, plus une partie des demandes recensées 
trouve des réponses, du moins partielles. L'ensemble de notre échantillon est composé de familles participant à des programmes d'intervention précoce ou dont les enfants sont scolarisés dans des services spécialisés.

Ces considérations ne doivent toutefois pas laisser penser que les parents constituent une population homogène. En effet, les demandes doivent être traitées sur une base individuelle. En d'autres termes, les équipes d'intervention ne peuvent pas prédire les demandes d'une famille particulière, de la mère et du père, à partir d'une expertise globale acquise avec l'expérience. Chaque famille est une situation nouvelle. Cette observation a été récemment confirmée par Ainge, Colvin et Baker (1998).

L'évaluation clinique menée lors de la passation de l'échelle avec les familles montre que la présentation d'une liste de demandes procure généralement une bonne entrée en matière avec les parents. Une mère a résumé les appréciations en nous disant: " $C^{\prime}$ est la première fois qu'on nous demande de quoi nous avons besoin pour nous et notre enfant. " Le père d'une fille de 12 ans atteinte de trisomie 21 s'exprimait ainsi: "Le problème, avant, c'est que personne ne s'intéressait à ce qu'on vivait. ”

\section{IMPLICATIONS}

Cette étape de l'évolution des idées en matière d'analyse du stress et du coping en déficience intellectuelle est fondamentale à nos yeux parce qu'elle remet en question nos habitudes de travail. Force est de constater en effet que les chercheurs et les praticiens restent fidèles à leurs vieilles habitudes, et cela malgré l'introduction du modèle conceptuel comme cadre de référence pour l'abord des familles. Ces habitudes consistent le plus souvent à imposer de l'extérieur un schéma d'analyse et à interpréter la réalité à partir de concepts. Ce rôle d'expert ne résiste pas à l'analyse pour deux raisons.

Premièrement, le travail avec les familles montre que celles-ci ont des demandes, le plus souvent non formulées, qui interfèrent plus ou moins fortement avec une action venant de l'extérieur. Ces demandes sont réelles. Elles ne sont pas limitées aux individus, mais elles touchent $l^{\prime}$ ensemble du système. Il s'agit de bien comprendre leur signification dans l'écologie familiale. Les demandes exprimées n'ont pas toutes le même statut en matière d'adaptation familiale. Il convient de les apprécier en fonction de trois dimensions. La première est le temps. Certaines demandes sont urgentes et doivent être satisfaites rapidement. Les problèmes financiers, la garde de l'enfant permettant d'assurer un répit aux parents et 
l'approche d'un grave trouble de comportement perturbant la vie quotidienne de la famille appartiennent à cette catégorie. D'autres exigent plus de temps : l'information sur la déficience ou la progression développementale d'un jeune enfant. La seconde dimension est l'appartenance de la demande à des niveaux différents de l'écosystème. D'une manière générale, les demandes dans le microsystème - les personnes en tant qu'acteurs de la vie quotidienne - sont plus faciles à combler que celles qui font intervenir des systèmes plus éloignés. C'est ainsi que l'exigence d'une meilleure collaboration avec l'école ou l'atelier suscitera plus $d^{\prime}$ investissement en temps et en énergie chez les professionnels. Enfin, on doit tenir compte de la dimension des possibilités de changements. Le caractère immédiat et tangible d'une requête ne facilite pas nécessairement l'intervention. Un conflit dans le couple, l'absence de partage des tâches éducatives, voire des signes de négligence envers l'enfant sont des phénomènes aisément identifiables pour tout professionnel ouvert aux réalités familiales. Cela ne signifie nullement que leur accès soit aisé. L'ensemble de ces éléments doit être pris en considération pour apprécier les demandes des familles. Le simple fait de présenter aux parents un instrument d'évaluation de leurs demandes n'est que la première étape d'un long processus de rencontre avec la réalité familiale. L'étude des conditions d'existence des familles, de leurs forces et de leurs faiblesses, permet ensuite de progresser dans la construction d'un plan d'intervention.

L'analyse des demandes familiales doit être considérée aujourd'hui comme le préalable à toute intervention. Cela est confirmé par les travaux les plus récents en la matière, qu'ils portent sur les réactions des professionnels impliqués (Bailey, Aytch, Odom, Symons, et Wolery, 1999) ou sur l'intégration communautaire des services d'aide précoce (Buysse, Wesley et Skinner, 1999).

La seconde raison pour laquelle le rôle d'expert joué par le chercheur ou le praticien ne tient plus la route émerge de l'étude des processus familiaux d'adaptation et plus particulièrement de la connaissance de deux mécanismes utilisés par les parents. Le premier est l'appropriation. C'est l'acquisition du sentiment de compétence et de confiance nécessaire pour participer adéquatement au processus d'adaptation et de soutien de la personne. Le second est l'autodétermination, l'habileté à se sentir capable d'assumer la responsabilité de décider, de préciser ses objectifs, ses rôles et ses attentes de service. La famille doit donc pouvoir indiquer comment elle entend se développer et participer à l'éducation et au développement de ses membres. L'étude de ces mécanismes fait partie intégrante d'une nouvelle perspective développée par Bouchard (1997) en matière d'intervention familiale: le partenariat. L'exposé d'ensemble de 
la théorie dépasserait le cadre du présent chapitre. Nous retiendrons que chercheurs et intervenants doivent se centrer sur les stratégies qui permettront aux familles de développer ce sentiment de compétence et de confiance en leurs ressources. Cette perspective en est encore au stade de l'expérimentation. Elle est très exigeante parce qu'elle demande une reformulation, parfois radicale, de nos manières de penser et d'agir.

Ces considérations montrent le chemin parcouru en trois décennies dans l'approche des familles d'enfants déficients intellectuels. La recherche s'est progressivement dégagée du modèle clinique considérant ces familles comme perturbées par la présence d'un enfant différent pour prendre en considération l'ensemble des variables forgeant le fonctionnement familial. Cette évolution façonne le travail des chercheurs et des intervenants pour comprendre le processus de l'adaptation. Notons toutefois que la perspective clinique consistant à rechercher les désorganisations dans les familles ayant un enfant déficient intellectuel continue à dominer la recherche. Dans un article récent, Helff et Glidden (1998) montrent en effet que la grande majorité des recherches se basent encore sur la conception des désorganisations familiales en raison de la présence d'un enfant déficient. Jusqu'en 1993, rares sont les travaux mettant en lumière les bénéfices retirés par des familles sur le plan de leur adaptation. L'orientation actuelle est en fait un nouveau paradigme, avec de nouvelles interrogations, dont les implications ne manqueront pas d'influencer la pratique. Fournir du soutien aux familles à partir de leurs demandes et de leur réalité quotidienne afin de les aider à s'adapter à leurs enfants, constitue en effet une entreprise toute différente que celle visant à les aider à faire face à leur douleur. Quels bénéfices les familles retirent-elles de la présence d'un enfant déficient au plan de leur adaptation? C'est pour tenter de répondre à cette question précise que nous avons développé notre modèle du coping.

\section{UN MODÈLE POUR L'ADAPTATION}

L'analyse des modèles théoriques rendant compte du stress et du coping nous met en présence de cinq grandes variables qui jouent un rôle prépondérant dans les familles d'enfants déficients intellectuels: les besoins, les réactions émotionnelles, les stratégies instrumentales et cognitives, les ressources et les attributions de signification. D'une manière générale, les différents auteurs travaillent avec ces variables de manière isolée. Au fur 
et à mesure de nos recherches, nous avons mis en lumière le fait que pareille situation n'était guère soutenable. C'est pourquoi nous avons développé une nouvelle approche englobant l'ensemble de ces variables.

\section{STRESS ET RESSOURCES}

À partir d'une analyse exhaustive des facteurs de stress et de ressources recensés dans les ouvrages scientifiques, nous avons mis au point une échelle d'évaluation permettant d'appréhender l'importance respective de ces variables dans une famille. Nous avons construit un premier instrument, que nous avons soumis à une analyse de validité auprès de 99 familles d'enfants déficients intellectuels âgés de 4 mois à 6 ans et 2 mois présentant tous un retard de développement significatif. Les étiologies sont variées, de nature exogène. Au fur et à mesure de la démarche expérimentale, nous avons affiné cette échelle pour retenir un ensemble de 51 items s'organisant autour de quatre dimensions: l'enfant, la famille, la mère et le père (Lambert, Piret, Scohy et Lalière, 1993; Lambert et Studer, 1998). Pour la famille, des études préliminaires nous ont fait retenir six variables: 1) le statut conjugal (couple parental, recomposé, parent unique); 2) la qualité des relations familiales; 3) le partage des tâches éducatives; 4) la prise en charge de l'éducation; 5) la qualité des relations sociales; 6) l'aisance financière. Le tableau 1 présente les variables retenues et le système de cotation.

Cet indice de difficultés-ressources (IDR) est composé de 19 variables, chacune étant mesurée sur une échelle à 3 points. La force des variables stressantes ressenties par les familles est directement proportionnelle au nombre de points relevés dans chaque dimension. À l'inverse, la qualité des ressources est appréciée par l'obtention d'un score IDR faible. Les analyses statistiques montrent que l'obtention d'un score égal ou inférieur à 19 (les items marqués * dans le tableau 1) est l'indicateur de la présence de ressources dans un milieu familial donné.

Dans la pratique, cet indice est bien accueilli par les professionnels. Il fait l'objet d'un recueil systématique de données dans chaque famille, immédiatement après la passation de l'échelle des demandes. Récemment, la pertinence de cet indice a reçu une confirmation dans l'étude de McBride et Peterson (1997). 


\section{TABLEAU 1}

Indice de difficultés/ressources (IDR)

\begin{tabular}{|c|c|c|c|}
\hline \multirow[b]{2}{*}{ Dimensions } & \multicolumn{3}{|c|}{ Niveaux d'évaluation } \\
\hline & 1 & 2 & 3 \\
\hline \multicolumn{4}{|l|}{ Enfant } \\
\hline Gravité de la déficience & Modérée * & Sévère & Profonde \\
\hline Handicaps associés & Aucun * & Un & Polyhandicap \\
\hline $\begin{array}{l}\text { Troubles de } \\
\text { comportement }\end{array}$ & Aucun * & Modérés & Graves \\
\hline \multicolumn{4}{|l|}{ Famille } \\
\hline Statut conjugal-couple & Intact ${ }^{\star}$ & Recomposé * & Parent seul \\
\hline Relations familiales & Bonnes * & Problématiques & Conflictuelles \\
\hline $\begin{array}{l}\text { Partage des tâches } \\
\text { éducatives }\end{array}$ & Bon * & Épisodique & Aucun \\
\hline $\begin{array}{l}\text { Prise en charge } \\
\text { éducative }\end{array}$ & Bonne * & Médiocre & Mise sous tutelle \\
\hline Relations sociales & Bonnes * & Indifférentes & Conflictuelles \\
\hline Problèmes financiers & Aucun * & Présents & Aide sociale \\
\hline \multicolumn{4}{|l|}{ Mère } \\
\hline Degré de scolarité & Supérieur * & Professionnel & Primaire-spécial ${ }^{1}$ \\
\hline Degré professionnel & Profession * & Arrêt & Pas de profession \\
\hline Santé mentale & Bonne * & Troubles & Maladie avérée \\
\hline Santé physique & Bonne * & Troubles & Maladie avérée \\
\hline Déficience mentale & Aucune * & Problèmes & Déficience avérée \\
\hline Négligence & Aucune * & Épisodique & Avérée \\
\hline \multicolumn{4}{|l|}{ Père } \\
\hline Degré de scolarité & Supérieur * & Professionnel & Primaire-spécial ${ }^{1}$ \\
\hline Degré professionnel & Profession * & Arrêt & Pas de profession \\
\hline Déficience mentale & Aucune * & Problèmes & Déficience avérée \\
\hline Négligence & Aucune * & Épisodique & Avérée \\
\hline
\end{tabular}

1. Niveau éducatif ne dépassant pas les six années d'école primaire (6 à 12 ans) ou atteint après une scolarité dans l'enseignement spécialisé non intégré (en règle générale de 6 à 18 ans). 


\section{LES RELATIONS AVEC L'ÉCOLOGIE FAMILIALE}

La recherche des facteurs de stress et de ressources n'a de sens que si elle est intégrée dans le fonctionnement global d'une famille. Le modèle de l'écologie familiale prédit que ces facteurs influencent deux grands aspects du milieu familial: l'environnement physique et les conduites éducatives. L'observation du milieu familial se réalise à partir d'un instrument dûment validé sur 108 familles d'enfants déficients (Lambert, Piret, Scohy et Lalière, 1994). Cet instrument comprend deux parties. La mesure du milieu physique couvre quatre domaines. La sécurité est un élément déterminant. L'échelle apprécie les caractéristiques suivantes: la non-accessibilité à des produits dangereux, l'absence d'agents polluants, la présence de commodités sanitaires élémentaires adaptées à l'âge de l'enfant, l'absence de structures dangereuses dans l'espace de vie des enfants et la propreté des lieux de vie. Les autres dimensions de l'évaluation du milieu physique sont la variété, la complexité et la réactivité des objets et des jouets proposés par les parents aux enfants. Cette partie de l'échelle d'observation comprend 43 items. Le milieu éducatif est apprécié par une échelle de 36 items touchant les conduites suivantes: la sécurité, les relations affectives et les interactions centrées sur l'apprentissage de l'enfant.

Nous avons mis en relation les résultats enregistrés à l'indice de difficultés-ressources (IDR) et l'observation du milieu de 109 familles d'enfants déficients intellectuels. Les données ont été soumises à des analyses de variance et de régression permettant de dégager les facteurs de prédiction. L'ensemble des dimensions évaluées par l'IDR exerce une influence significative sur certains aspects de la qualité de l'environnement éducatif fourni aux enfants.

L'obtention d'un score IDR élevé, soit supérieur à 21, est liée de manière significative à des résultats inférieurs dans les domaines suivants: la sécurité du milieu physique, la variété et la complexité des jouets et des objets présentés aux enfants, les conduites d'apprentissage présentées par les parents et les conduites affectives des mères. Il existe donc des relations étroites entre l'importance du stress global ressenti par les familles et les caractéristiques physiques et sociales des milieux éducatifs, ces dernières se détériorant avec l'accroissement du stress. À l'inverse, la qualité du milieu est directement liée à la force des ressources familiales.

La dimension familiale dans son ensemble agit nettement sur la qualité du milieu éducatif. Plus les scores IDR sont bas, meilleures sont la variété et la réactivité du milieu physique 
et les conduites éducatives des mères et des pères. Nous n'avons pas dégagé de variables significatives à l'intérieur de cette dimension "famille». C'est donc l'ensemble de la constellation comprenant des éléments du microsystème, de l'endosystème et de l'exosystème qui agit sur la qualité du milieu proposé à l'enfant.

L'indice difficultés/ressources ne constitue qu'un élément permettant d'apprécier les variables influençant le coping. Cet indice reste trop général dans la mesure où la présence de ressources reste trop fondée sur l'absence de grands facteurs de stress. Nous avons poursuivi l'analyse des ressources au sein de l'échantillon en mettant en lumière neuf grandes ressources en relation directe avec la qualité du milieu physique et éducatif des enfants.

Voici les ressources trouvées chez les parents et dont la présence permet d'offrir aux enfants déficients une bonne qualité éducative:

- planifier l'horaire de vie de l'enfant et de la famille;

- guider l'enfant vers des réponses médicales adéquates;

- réaliser des activités pour soi-même;

- présenter une image personnelle positive;

- respecter les contrats établis avec les services;

- entretenir des relations constructives avec les milieux de vie de l'enfant;

- utiliser spontanément les ressources du milieu (services, thérapeutes);

- montrer une motivation à trouver ou à garder un travail;

- posséder un réseau social stable en dehors de la famille.

À nouveau, ces ressources se trouvent dans tous les sous-systèmes de l'écologie familiale. Ces observations montrent l'importance pour les intervenants d'approcher la famille comme un écosystème et de privilégier, dans l'appréciation de la situation, toutes les ressources psychologiques, matérielles et sociales que les familles considèrent comme importantes.

\section{DES STRATÉGIES DE COPING}

Les analyses qui précèdent ne doivent pas faire penser que le coping familial est un processus figé dépendant notamment d'une bonne évaluation des éléments de stress et de ressources présents dans le milieu. 
Éléments centraux dans l'étude de l'adaptation familiale et des réactions au stress, les stratégies de coping développées par les parents d'enfants déficients intellectuels font l'objet d'études de plus en plus nombreuses basées sur divers modèles théoriques. Toutefois, nous n'avons pas trouvé dans les ouvrages scientifiques d'écrits synthétisant l'ensemble des stratégies utilisées par les parents. Face à ce manque d'approche unitaire, nous avons développé un questionnaire destiné à définir les stratégies de coping. Nous nous basons sur deux références conceptuelles précises.

D'abord, la distinction entre les stratégies cognitives et les stratégies instrumentales. Les premières regroupent les réflexions, les idées, les processus de catégorisation et de référence exprimés par les parents avant et pendant le processus de coping et les secondes, toutes les actions que les parents entreprennent pour faire face à une situation problématique. Il serait toutefois illusoire d'accorder à cette distinction une valeur heuristique universelle. En effet, il apparaît que les deux groupes de stratégies sont en interactions. Pour la recherche, il est cependant utile d'opérer la distinction entre ce que les parents font lorsqu'ils sont confrontés à une situation difficile et ce qu'ils en pensent sans nécessairement traduire ces pensées en conduites observables par le chercheur. Cette différenciation est en outre congruente avec le modèle de coping classique développé par Lazarus et Folkman (1984) classant les stratégies selon leur fonction: centrées sur les émotions ou axées sur la résolution.

Ensuite, la distinction, présentée par Perrez et Reicherts (1992), entre les différentes stratégies de coping en fonction des buts qu'elles visent. Ils identifient ainsi des stratégies destinées à agir sur la situation (action, échappement, retrait, passivité), sur la représentation (recherche et suppression d'information) et sur l'évaluation (modification des intentions et des buts, réévaluation de la situation).

Nous avons conçu un questionnaire comportant 32 items et couvrant 17 stratégies cognitives et 15 instrumentales. L'analyse de la saturation, réalisée à partir des publications scientifiques, montre que ce questionnaire regroupe les stratégies identifiées par les chercheurs en déficience intellectuelle jusqu'en 1994.

Nous demandons aux parents de penser à une situation concrète et récente à laquelle ils ont été confrontés avec leur enfant déficient, puis d'analyser cette situation en fonction de ce qu'ils ont pensé ou de ce qu'ils ont fait pour tenter de résoudre le problème. Afin de donner un caractère plus général 
à leurs réponses, nous demandons également aux parents ce qu'ils font et ce qu'ils pensent dans d'autres situations qu'ils jugent problématiques. Voici quelques items de l'échelle:

- Il est important que mon conjoint/compagnon et moi trouvions une solution commune.

- J'ai confiance en moi et en mes capacités pour trouver une solution.

- Je peux trouver certaines situations drôles et en rire.

- Je vois une difficulté comme un défi.

- Je me dis que je contrôle la situation.

- Je demande de l'aide à mon conjoint ou mon compagnon.

- Je demande de l'aide à des professionnels.

- Je discute avec des amis pour avoir des conseils.

- Je m'organise pour trouver l'aide financière.

- Je ne cherche pas d'information supplémentaire.

- Mes croyances m'aident à mieux gérer les défis.

L'échantillon est composé de 178 parents (98 mères et 80 pères) pour 98 familles, soit 18 familles monoparentales et 80 familles formées des deux parents. Toutes ont un enfant présentant une déficience intellectuelle avérée, c'est-à-dire un quotient de développement ou un quotient intellectuel inférieur à 75 , critère d'éligibilité pour recevoir les services d'un centre d'intervention précoce. Les enfants sont âgés de trois mois à sept ans. Les parents ont accepté de répondre à un ensemble de questions structurées portant sur les besoins, les réactions émotionnelles et les stratégies utilisées pour faire face aux problèmes que posent l'éducation de leur enfant et leur fonctionnement dans le système écologique général. Les instruments ont été adaptés à partir des travaux de différents chercheurs (Lanners, 1997; Perrez et Reicherts, 1992; Patterson, 1993). Les résultats ont été soumis à diverses analyses factorielles (en composantes principales avec rotation varimax).

Les analyses factorielles des résultats font ressortir huit grandes stratégies de coping utilisées par les parents: avoir confiance en soi, développer des pensées positives, rechercher de l'aide professionnelle, rechercher de l'aide au sein de la famille, rechercher de l'aide dans l'entourage immédiat (voisins, amis), rechercher la cohérence au sein du couple, exercer un contrôle sur la situation et rechercher de l'aide spirituelle. Les données montrent des différences entre les pères et les mères. 
Les pensées positives sont les stratégies les plus couramment utilisées par les mères. Ces dernières tentent ensuite de rechercher de la cohérence au sein du couple, soit en discutant avec leur conjoint, soit en lui demandant de l'aide. Les mères se reposent moins sur les professionnels, elles se basent plus sur leurs capacités. Elles se motivent alors en attribuant un sens à la difficulté et tentent de trouver son origine. Lorsqu'elles ne parviennent pas à contrôler la situation, elles demandent de l'aide à d'autres membres de la famille ou à des amis.

La stratégie la plus fréquente chez les pères est la recherche de cohérence au sein du couple. Ils estiment en effet très important le fait de discuter avec leur conjointe et de leur demander leur aide face à une difficulté. Contrairement aux mères, ils font moins confiance en leurs capacités et trouvent moins de motivation en leurs propres ressources pour affronter le problème. La recherche d'une aide professionnelle est une stratégie fréquemment utilisée.

La lecture des stratégies met en lumière des processus complexes déterminés à la fois par les conditions de vie des familles, la personnalité de chaque parent, le réseau social, les problèmes spécifiques posés par l'enfant déficient et le résultat des actions que les familles ont entreprises pour y faire face. Il est important de savoir que les réactions émotionnelles des parents doivent retenir l'attention des professionnels. Comme le montrent en effet les différents modèles théoriques utilisés actuellement pour rendre compte du coping, les émotions parentales jouent un rôle important dans l'activation des stratégies.

Pour analyser ce rôle, nous avons présenté aux parents une échelle de réponses émotionnelles en leur demandant de citer les émotions les plus fréquemment ressenties lors de la présence d'une difficulté dans la famille. Quatre grandes réactions dominent le tableau et expliquent à elles seules $67 \%$ de la variance. Face à une situation difficile avec leur enfant, les parents éprouvent globalement deux réactions positives (se sentir à l'aise et avoir le sentiment d'être soutenu par un autre membre de la famille) et deux réactions négatives (se fâcher et le fait de se sentir dépassé par la situation). En fait, ces catégories regroupent un ensemble de réactions émotionnelles diversifiées. L'émotion prédominante est de se sentir soutenu; le conjoint est le plus fréquemment cité comme facteur de support. Viennent ensuite les émotions regroupées sous la catégorie "se sentir à l'aise » : rester calme, se sentir de bonne humeur, ne pas s'énerver. Puis, une fréquence d'apparition pratiquement identique, le fait de se fâcher ou de s'énerver. Le sentiment d'être dépassé par la situation (être anxieux, dépressif, triste) est le moins fréquent. Les analyses statistiques montrent 
que l'âge des parents n'a pas d'influence significative sur ces réactions émotionnelles. L'âge de l'enfant et la durée de l'intervention proposée aux parents n'exercent pas non plus d'influence significative.

$\mathrm{Au}$ premier abord, il peut paraître surprenant que l'intervention éducative prenant place au domicile des familles et surtout sa durée n'influencent guère les réactions émotionnelles des parents. Ce résultat concorde cependant avec les données d'un ensemble de recherches qui montrent que l'intervention éducative dans la famille ne s'accompagne pas nécessairement d'une transformation des réactions émotionnelles face à des situations quotidiennes problématiques, à moins que cette intervention porte directement sur cet aspect (Barnard, 1997). C'est d'ailleurs un des défis majeurs des nouvelles approches en matière d'intervention que de doter les parents des mécanismes d'appropriation de connaissances et de stratégies efficaces leur permettant de modifier progressivement leurs réactions psychologiques face aux difficultés éducatives.

Les seules différences sont observées entre les pères et les mères. Face à des situations difficiles, les pères se sentent généralement plus à l'aise que les mères, mieux soutenus et moins dépassés par les événements. Nous expliquons ce résultat par le fait que les pères s'impliquent moins dans les soins et la prise en charge quotidienne des enfants déficients intellectuels, surtout lorsqu'ils sont jeunes. Nous avons validé cette constatation avec d'autres groupes de pères. Elle est le résultat d'un réseau complexe d'influences culturelles (le rôle des pères dans l'éducation des jeunes enfants), psychologiques (d'une manière générale, les pères acceptent moins bien la déficience chez leur enfant dès que celui-ci est plus âgé et entre notamment dans sa période scolaire) et administratives (les services d'aide précoce centrant généralement leur action sur les mères) (Lambert et Studer, 1998).

Ces quelques données ne sont qu'un aperçu de la diversité des stratégies auxquelles recourent les familles pour faire face à la présence d'un enfant déficient et à son éducation. Ces résultats n'illustrent qu'imparfaitement deux réalités. Premièrement, la mise en action de ces stratégies varie selon un nombre impressionnant de facteurs, parmi lesquels il est nécessaire de prendre en compte les variables définies dans l'IDR. Deuxièmement, chaque famille apparaît comme une réalité difficilement réductible à une moyenne statistique. En d'autres termes, le champ de l'éducation familiale en déficience intellectuelle demande de la part des professionnels une approche individualisée de chaque famille. 


\section{L'ADAPTATION FAMILIALE: UN SYSTÈME COMPLEXE}

À partir du moment où les besoins, les facteurs de stress et de ressources des parents et leurs stratégies de coping sont définis, il est nécessaire de passer à l'étape suivante de l'analyse et de voir comment ces différents éléments des réactions familiales s'articulent.

Tout d'abord, il est nécessaire de distinguer le type de besoins de leur quantité. Le nombre de besoins exprimés par une famille induit un phénomène de surcharge émotionnelle. C'est particulièrement le cas des besoins non satisfaits en soutien informel et formel. Le type de besoins est également important à prendre en compte. Certaines demandes sont considérées par les parents comme prioritaires. Si elles ne sont pas satisfaites, elles perturbent l'adaptation.

Les parents ne sont pas démunis face à leurs besoins. Une stratégie s'impose par sa fréquence et surtout par son efficacité: la confiance en soi. Elle a pour conséquence la réduction globale des demandes et la satisfaction de besoins précis comme la recherche d'information et de soutien. Toutefois, certaines stratégies créent à leur tour des besoins.

Quatre stratégies utilisées par les parents dans le processus d'adaptation font apparaître des besoins nouveaux. La recherche de l'aide au sein de la famille accroît les besoins en information. Par exemple, une mère sollicite l'aide de sa fille âgée de sept ans pour différentes tâches. Elle se heurte tout d'abord au refus de son enfant, celle-ci prétextant que son frère déficient, plus âgé, n'est pas sollicité de la même manière. La mère doit alors trouver les éléments de réponse lui permettant d'expliquer à sa fille la déficience de l'aîné et son incapacité à effectuer certaines tâches. Comme le disait cette mère: "Comment puis-je expliquer à un enfant de sept ans ce qu'est l'autisme? ”

La recherche d'une aide chez les professionnels, une stratégie instrumentale courante, fait augmenter le nombre global de besoins et accroît spécifiquement les demandes en soutien. Plus les parents demandent de l'aide aux professionnels, plus ils ressentent de besoins. Cette situation apparemment paradoxale peut s'expliquer de la manière suivante. Lorsque les parents font appel à des professionnels, c'est vraisemblablement parce qu'ils ne peuvent plus faire face à la situation problématique posée par leur enfant. Ils observent que leurs ressources sont insuffisantes. L'introduction des professionnels dans l'univers familial fait apparaître d'autres besoins, les parents se déchargeant en quelque sorte de leurs demandes. Nous savons également que les professionnels 
peuvent créer des besoins, notamment en ne répondant pas aux questions des parents et en se confinant dans des rôles d'experts (Bouchard, 1997). L'importance d'une éducation familiale mettant l'accent sur les mécanismes d'appropriation des parents s'impose comme solution partielle à cette situation (Mahoney et Bella, 1997).

Le fait d'exercer un contrôle va de pair avec l'augmentation des besoins financiers. Plus les parents tentent de contrôler la situation, plus ils expriment des besoins financiers pour couvrir les thérapies, les équipements spéciaux ou les soins pour leur enfant. Il apparaît que le fait de rechercher activement des renseignements et des solutions entraîne des dépenses supplémentaires. Le processus est identique pour les stratégies que nous appelons " motivationnelles".

La majorité des parents ne sont pas démunis face au poids des réactions émotionnelles. Dans notre échantillon, les stratégies les plus importantes permettant aux parents d'accroître leurs réponses positives sont la recherche de cohérence au sein du couple, l'exercice d'un contrôle et les croyances spirituelles.

La recherche de cohérence dans le couple s'exerce de multiples manières: soutien, prise de décision en commun, partage du vécu émotionnel, accord sur les décisions éducatives. Cela renforce le sentiment d'être soutenu, lequel à son tour accroît la probabilité d'apparition ultérieure d'une recherche de cohérence. Dans notre échantillon, les parents font une part belle aux systèmes de croyance laïque ou religieuse comme stratégie cognitive leur permettant d'affronter les difficultés. Ces croyances ont depuis longtemps été reconnues comme des ressources importantes dans les familles d'enfants déficients intellectuels (Lambert et Lambert-Boite, 1993). Enfin, l'exercice d'un contrôle direct sur la situation (demande d'aide, participation à une association de parents, développement d'une action précise face à un stress) renforce le développement de réactions émotionnelles positives.

Ces relations ne sont que quelques exemples du réseau complexe des interactions qu'entretiennent entre eux les besoins, les réactions émotionnelles et les stratégies. Nous ne pouvons plus actuellement accorder de crédit aux affirmations qui prévalaient il y a encore une décennie quant au fonctionnement familial, à savoir que les besoins avaient un impact négatif sur le vécu émotionnel des parents, que ceux-ci étaient 
des victimes consentantes, qu'ils étaient incapables de coping ou que les stratégies de coping amenaient automatiquement une réduction des besoins. La réalité de l'adaptation familiale est infiniment plus nuancée.

Un fait s'impose au terme de cette recherche empirique portant sur un nombre assez important de familles, $c^{\prime}$ est qu'elle présente les tendances centrales des phénomènes les plus suggestifs. Ces grandes tendances ne doivent pas faire perdre de vue la nécessité d'analyser la situation de chaque famille. En effet, l'organisation générale de notre modèle de l'adaptation permet son application à la réalité unique de chaque famille. Toute généralisation à l'ensemble des familles ayant un enfant déficient intellectuel est hâtive et n'offre vraisemblablement qu'une vision peu exacte de la réalité individuelle. Fort heureusement, d'une manière générale, la recherche en matière d'étude familiale en déficience intellectuelle a tendance à privilégier les méthodes dites à cas unique pour marquer l'aspect spécifique des réactions adaptatives de chaque famille (Krauss, 1997).

Cette étude empirique n'est qu'une étape dans le développement de notre modèle de l'adaptation familiale. Plusieurs contraintes théoriques et méthodologiques en limitent actuellement la validité. D'abord, des recherches supplémentaires doivent être menées pour préciser le rôle exact que jouent les métastratégies et plus particulièrement les facteurs motivationnels. Ensuite, les stratégies cognitives et instrumentales identifiées dans cette recherche ne sont pas nécessairement les seules. Si notre questionnaire est fondé quant à la validité du construit, sa validité de contenu demande encore à être soumise à l'épreuve des faits. Seule la multiplication des observations permettra de dresser la liste de ces stratégies et d'appréhender leur impact sur la réalité individuelle des familles. En outre, le fait que l'étude porte sur des familles d'enfants de trois mois à sept ans et qu'elle soit de caractère transversal empêche toute généralisation à l'ensemble du phénomène de l'adaptation familiale. Il est cependant vraisemblable que le tableau des interactions observées entre les besoins, les émotions et les stratégies évolue avec le temps, sous l'influence de variables comme l'âge de l'enfant et des parents, l'intervention d'autres structures éducatives dans la vie de l'enfant déficient, les transformations des milieux de vie et l'expression de plus en plus affirmée des déficiences et des compétences des enfants. Enfin, l'adaptation familiale étant un phénomène évolutif, seul le recours à des analyses longitudinales permettra de mieux en saisir les processus. 


\section{UNE PISTE À PRIVILÉGIER: LE COPING CHEZ LES ENFANTS DÉFICIENTS}

L'étude de l'adaptation des familles d'enfants déficients intellectuels est en plein essor. De nombreuses voies s'offrent aux chercheurs. Citons, par exemple, le rôle des pères dans le processus de coping et l'éducation de leurs enfants déficients (Lambert et Studer, 1998), les stratégies mises en place par des mères elles-mêmes déficientes intellectuelles (Feldman et Walton-Allen, 1997) et l'impact familial de troubles envahissants du comportement (Stores, Stores, Fellows et Buckley, 1998). Nous avons choisi de présenter un thème d'avenir pour la compréhension des phénomènes de l'adaptation familiale, mais pour lequel nous manquons de données empiriques susceptibles de fonder une pratique cohérente.

Le stress et le coping chez les enfants non déficients sont des domaines bien documentés. D'une manière générale, les chercheurs ont suivi les courants théoriques traversant l'analyse de ces phénomènes chez les adultes, notamment en mettant l'accent sur l'étude du stress quotidien, les différences individuelles et les relations entre le coping et la compétence sociale (par exemple, Causey et Dubow, 1992). Par contre, le développement des stratégies de coping chez les enfants dans le cadre familial reste un univers peu connu. L'étude de Hardy, Power et Jaedicke (1993) est une des premières tentatives structurées visant à mettre en relation les styles parentaux et les formes de coping d'enfants de 10 ans dans leur adaptation quotidienne. À notre connaissance, cela ne s'est pas encore fait dans le champ de la déficience intellectuelle. Une recherche exhaustive dans les bases de données internationales nous a fait découvrir deux travaux abordant cette problématique. Le premier n'a qu'une relation très mince avec le coping envisagé dans une perspective familiale. Il traite des relations entre les facteurs de stress et la dépression chez des enfants autistes (Ghaziuddin, Alessi et Greden, 1995). Le second étudie la perception qu'ont les mères des difficultés de coping de leurs enfants atteints de trisomie 21 (Greaves et Poole, 1996). Nous disposons de notre côté de données cliniques recueillies auprès de familles d'enfants déficients âgés en moyenne de sept ans. Nous avons choisi de les présenter brièvement en guise de conclusion à ce chapitre parce qu'elles nous semblent illustrer à souhait les difficultés auxquelles les chercheurs font face lorsqu'ils abordent le coping chez les enfants déficients, et mettre en lumière les enjeux futurs de la recherche en adaptation familiale dans la déficience intellectuelle. 
La première difficulté est d'ordre méthodologique. Les recherches sur les stratégies de coping des enfants non déficients font appel à des inventaires de conduites, des questionnaires ou autres instruments standardisés. Les différentes dimensions du coping sont étudiées à partir des résultats à ces échelles. En outre, dès l'âge de six ans, les enfants peuvent répondre à des questionnaires d'auto-évaluation car ils sont généralement capables de répondre à des questions sur les événements de leur vie quotidienne. Lorsque les chercheurs utilisent des méthodes d'observation des conduites dans les milieux de vie des enfants, ils se servent de répertoires validés sur le plan développemental. Nous ne disposons pas $\mathrm{d}^{\prime}$ instruments semblables pour les enfants déficients intellectuels. D'une part, le recours à toute forme d'auto-évaluation est difficile, voire impossible compte tenu des difficultés cognitives et langagières de nombreux enfants. D'autre part, les inventaires des stratégies de coping observées chez les enfants non déficients requièrent des compétences affectives, cognitives et sociales non maîtrisées par la plupart des enfants déficients.

Hardy, Power et Jaedicke (1993) ont développé un système de classification des stratégies de coping utilisées par des enfants de 10 ans. Ils dénombrent ainsi 12 stratégies. À partir d'observations sur le terrain, nous avons tenté de classer les stratégies utilisées par des jeunes déficients intellectuels modérés de 14 à 16 ans. Nous avons relevé 42 conduites chez 18 jeunes. Ces conduites font appel à 5 stratégies différentes. Leur distribution montre que 38 d'entre elles appartiennent en fait à trois catégories de conduites de coping: l'acceptation (l'adaptation à la pression directe ou l'adoption d'une conduite en accord avec une requête), la décharge émotionnelle (crier, pleurer, agresser) et le désengagement comportemental (le retrait de la situation).

Pour pallier ces écueils, une solution consiste à demander aux parents de décrire les stratégies utilisées par leurs enfants déficients dans les activités familiales. On se heurte alors à un obstacle méthodologique supplémentaire: le manque d'instruments valides permettant d'atteindre cet objectif.

L'étude de Greaves et Poole (1996) illustre ce fait. Ces chercheurs ont utilisé un instrument très populaire pour l'analyse du stress, le Parenting Stress Index. L'échelle comprend le sous-domaine "Adaptation/Plasticité " censé être un facteur permettant de mesurer les conduites de coping chez les enfants. Les résultats obtenus montrent que ce sous-domaine n'est pas valide pour des enfants déficients intellectuels. Il ne 
s'agit pas d'une structure unidimensionnelle. Les manifestations comportementales particulières des enfants face à des stresseurs exigent en fait une description individuelle des conduites de coping.

Nous ne disposons donc pas d'un inventaire standardisé des réactions de coping utilisable avec des enfants déficients intellectuels. Le fait de recourir aux interviews des parents pour obtenir une description de ces conduites chez leurs enfants nous place devant une troisième difficulté, de nature conceptuelle. Dans notre modèle du coping, certaines stratégies adoptées par les parents sont déjà le résultat de leur adaptation à des conduites de coping de leurs enfants. Voici un exemple.

Une mère utilise de préférence une stratégie instrumentale consistant à discuter en famille pour trouver une solution à une difficulté. Désirant prendre un peu de temps pour ellemême, cette mère est confrontée au problème de garde de son enfant durant les soirées. Toutes les tentatives pour trouver une gardienne se sont soldées par des échecs, sa fille de huit ans refusant systématiquement d'être gardée par une personne étrangère au milieu familial. En fait, l'observation de l'enfant montre qu'elle développe des stratégies de coping face aux routines familiales: elle ne pose pas de problèmes de comportement et s'adapte de manière très prévisible aux faits quotidiens à condition qu'ils se déroulent dans un climat sécurisant. Par contre, toute rupture de ces routines, notamment la présence de personnes étrangères au milieu, entraîne des réactions d'opposition.

Le caractère nécessairement interactif des conduites de coping chez les parents et les enfants n'est pas saisissable par la seule observation des conduites parentales. Les recherches futures devront s'astreindre à dresser des inventaires du coping chez les enfants déficients intellectuels. Très souvent, des enfants déficients intellectuels sont décrits comme "difficiles" ou "perturbés " parce qu'ils ne réagissent pas de manière adaptée à des événements de leur vie quotidienne. Nous posons comme hypothèse que ces réactions sont en fait l'expression des difficultés à produire un coping adapté.

Nos observations montrent qu'une grande partie des enfants déficients réagissent aux stresseurs selon un registre limité où la passivité et les conduites émotionnelles spectaculaires (crier, pleurer, agressivité) sont dominantes. Face à quelque chose ou à quelqu'un qu'ils n'aiment pas ou qui leur fait peur, des enfants réagissent par la passivité. Plutôt que de 
s'adapter aux exigences de la situation nouvelle, ces enfants utilisent une stratégie d'évitement. Ce qui peut être considéré comme une réaction de coping de l'enfant accroît en fait le stress de certains parents. Dans le même ordre d'idée, si certains enfants expriment leur difficulté d'adaptation à des modifications de leur vie quotidienne par une opposition parfois violente, c'est peut-être qu'ils perçoivent leur manque de contrôle sur des événements modifiant leur routine ou perturbant leur sécurité affective.

Les recherches en éducation familiale montrent que les enfants sont des acteurs à part entière dans la dynamique familiale. Les types de supports offerts par les parents à leurs enfants, les techniques de contrôle qu'ils utilisent pour guider les conduites de leurs enfants, et leur capacité à organiser l'environnement sont également façonnés par les conduites des enfants. L'étude du coping chez l'enfant déficient intellectuel est une voie à privilégier pour comprendre la complexité de l'adaptation familiale. Les données ainsi recueillies permettront de mieux saisir les relations entre les styles familiaux, les compétences et les difficultés cognitives, affectives et sociales des enfants déficients intellectuels. Tout modèle de coping destiné à analyser les processus adaptatifs des familles demande l'introduction d'une dimension importante: le point de vue de l'enfant déficient intellectuel. 



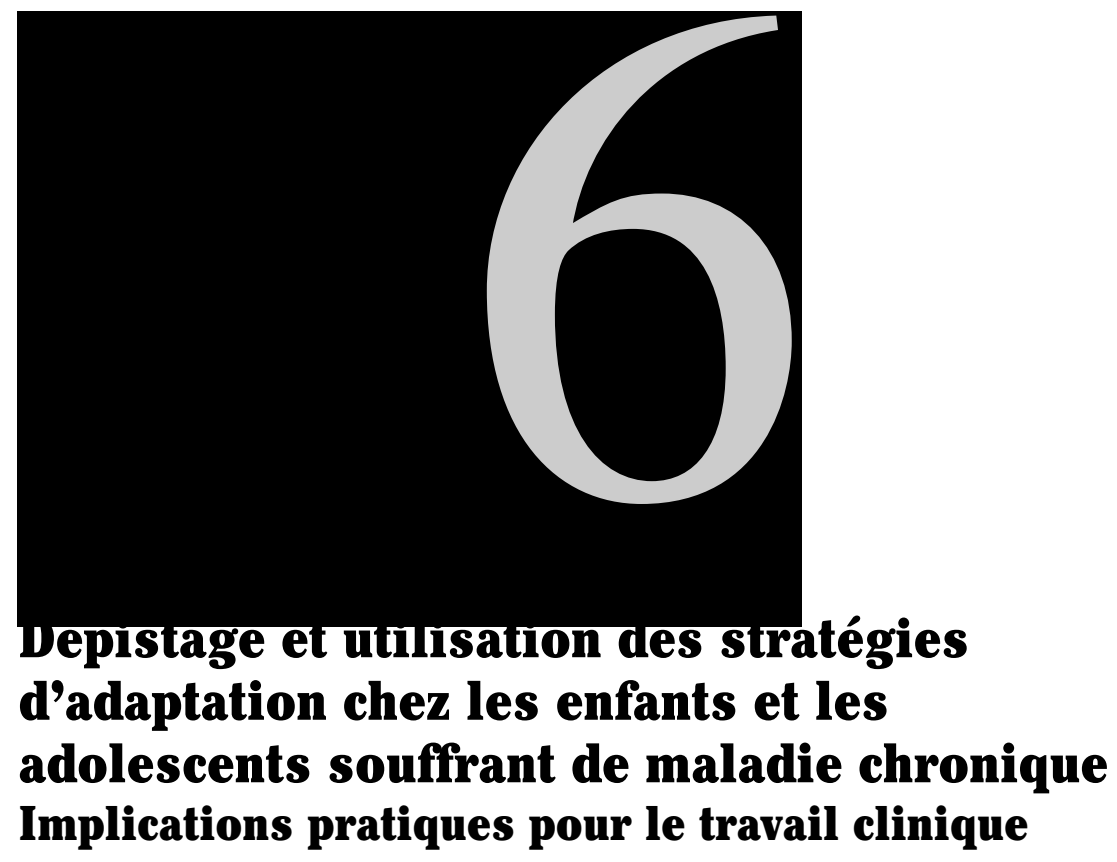

ANTHONY SPIRITO

Department of Psychiatry and Human Behavior

Brown University School of Medicine

Providence (Rhode Island)

DEIDRE L. DONALDSON

Department of Psychiatry and Human Behavior Brown University School of Medicine

Providence (Rhode Island) 



\begin{abstract}
Durant les années 1980, l'amélioration remarquable des traitements médicaux destinés aux maladies chroniques et mortelles a suscité un grand intérêt à mieux comprendre comment les enfants arrivent à surmonter une telle situation. Cet intérêt des chercheurs et des cliniciens prend son origine dans l'hypothèse voulant que le processus adaptatif, la manière de faire face à une situation difficile (coping), peut médiatiser les effets potentiellement négatifs de la maladie chronique. Notre groupe de recherche a voulu voir: 1) si les enfants atteints d'une maladie chronique utilisent différentes stratégies d'adaptation pour gérer divers aspects stressants de la maladie et des traitements; 2) si des changements surviennent dans la manière de faire face au cours de la maladie; et 3) si les enfants qui s'adaptent le mieux à leur maladie utilisent de manière constante certaines stratégies d'adaptation. À cette époque-là, il n'existait aucune mesure standardisée pour évaluer l'adaptation des enfants. Par conséquent, les premiers efforts de notre groupe de recherche se sont concentrés sur le développement d'une telle mesure. La mise au point d'une mesure de dépistage semblait appropriée puisque les chercheurs-cliniciens étudiant l'adaptation s'intéressaient non seulement à certains facteurs comme les facteurs familiaux, mais aussi aux mécanismes individuels d'adaptation chez les enfants traités en pédiatrie.
\end{abstract}

Dans ce chapitre, nous résumons les différentes étapes qui ont permis l'élaboration d'un instrument de mesure appelé le Kidcope et les premières études de fiabilité et de validité. Nous présentons d'abord des résultats obtenus auprès d'enfants en santé, afin de fournir aux lecteurs une vue d'ensemble des différences possibles dans l'utilisation des stratégies d'adaptation selon l'âge, le sexe et les stresseurs. Nous décrivons ensuite les stratégies d'adaptation utilisées par des enfants faisant partie d'échantillons psychiatriques. Toutes ces études nous ont procuré des données préliminaires, lesquelles nous ont par la suite amenés à étudier les enfants atteints de maladie chronique. Ces études sont décrites dans ce chapitre. Finalement, nous concluons en rendant compte de quelquesunes des difficultés auxquelles on fait face lorsqu'on utilise des mesures brèves de dépistage pour évaluer l'adaptation. 


\section{LE KIDCOPE}

Le Kidcope a été conçu pour évaluer une grande variété de stratégies cognitives et comportementales, tout en étant suffisamment bref pour être utilisé dans un contexte de pratique clinique. De manière plus précise, les énoncés de l'échelle évaluent 10 stratégies d'adaptation cognitives et comportementales utilisées par les enfants et les adolescents. Nous savons que ces stratégies d'adaptation ne conviennent pas à toutes les situations, et qu'il y aura des cas où les chercheurs devront ajouter des énoncés afin d'aborder des questions spécifiques (stratégies d'adaptation face à la douleur lorsque celle-ci est le sujet d'étude). Le Kidcope demande aux répondants non seulement de rapporter la fréquence d'utilisation de chacune des stratégies déployées en réponse à un stresseur (" pas du tout ", " parfois ", " souvent " et " presque toujours "), mais aussi d'évaluer l'efficacité de leur utilisation respective ("pas du tout», "un peu ", "plutôt ", "assez ", " très »). Des versions différentes du Kidcope ont été développées pour convenir à l'âge des enfants; pour les plus jeunes (de 7 à 12 ans) et pour les plus vieux (de 13 à 18 ans).

Il existe diverses manières de conceptualiser et d'organiser les nombreuses catégories de stratégies adaptatives. On retrouve les stratégies centrées sur le problème versus celles centrées sur l'émotion (Lazarus et Folkman, 1984), les stratégies actives versus les stratégies passives et, enfin, les stratégies d'approche versus les stratégies d'évitement. Le tableau 1 présente les catégories d'adaptation retenues par le Kidcope. Les énoncés ne sont pas organisés selon une structure ordonnée puisqu'on $\mathrm{s}^{\prime}$ attendait à ce que la fonction de chacune des stratégies d'adaptation varie selon la situation évaluée. Cependant, dans la plupart des cas, les stratégies centrées sur le problème, actives et d'approche, peuvent comprendre la restructuration cognitive, la résolution de problème et le soutien social. Les stratégies centrées sur l'émotion peuvent quant à elles comprendre la régulation émotionnelle et le soutien social, alors que les stratégies passives et évitantes peuvent inclure la distraction, le retrait social, la pensée magique et la résignation. La version du Kidcope pour les enfants plus âgés (13-18 ans) est présentée au tableau 2. Les réponses des enfants n'ayant pas de problème de santé ont été examinées lors du développement initial de l'instrument de mesure. Non seulement les études ont-elles été nécessaires pour le développement de l'instrument mais elles ont également permis de vérifier les propriétés psychométriques de la mesure et, surtout, d'établir des données normatives auxquelles l'adaptation des enfants souffrant de maladie chronique pouvait être comparée. La fiabilité du Kidcope a été vérifiée lors d'une étude sur les jeunes enfants (Spirito, Stark, Grace et Stamoulis, 1991). Lors du retest, 
on demandait aux mêmes enfants comment ils s'étaient adaptés aux problèmes qu'ils avaient décrits une ou deux semaines plus tôt. Les coefficients phi et les pourcentages de concordance étaient tous deux calculés pour chacun des énoncés. Il ne fut pas surprenant de constater que la plupart des coefficients phi et des pourcentages de concordance étaient plus élevés lorsqu'il ne s'était passé qu'une semaine plutôt que deux entre le test et le retest.

Bien que la concision du Kidcope puisse être attrayante pour les chercheurs, elle comporte tout de même quelques restrictions. Étant donné que chaque énoncé représente une stratégie d'adaptation individuelle (distraction, résolution de problème), la fiabilité test-retest a dû être calculée pour chaque énoncé plutôt qu'à partir du score global. La fiabilité des coefficients, bien que meilleure que prévue, était plus basse que celle des échelles incluant des énoncés multiples. De plus, le Kidcope repose sur un modèle développemental de l'adaptation (process model) qui suppose une faible stabilité des individus dans le temps (Lazarus et Folkman, 1984). Ainsi, de fortes corrélations test-retest peuvent se révéler impossibles à obtenir puisque les stratégies d'adaptation face à un même problème peuvent varier dans le temps (Mash et Terdal, 1988). D'autres types de mesures de fiabilité (consistance interne) peuvent être plus appropriées pour des mesures développementales (process measures). Toutefois, les coefficients alpha ne sont pas appropriés pour cette mesure puisque chacun des énoncés du Kidcope sont indépendants. Bien que cela soit un point faible de la mesure, les résultats de la fiabilité test-retest sont similaires à ceux d'autres études utilisant des mesures développementales (voir Spirito, Stark et Williams, 1988).

La validité du Kidcope a été évaluée par des comparaisons avec des mesures de l'adaptation préalablement standardisées telles que le Coping Strategy Inventory (CSI, Tobin, Holroyd et Reynolds, 1984) et le Adolescent-Coping Orientation for Problem Experiences Inventory (ACOPE, Patterson et McCubbin, 1983). Toutes les analyses de validité pour les mesures du Kidcope ont été obtenues à partir d'un stresseur choisi par la personne elle-même. Puisque plusieurs des sous-échelles au CSI étaient plutôt similaires à huit des catégories d'adaptation du Kidcope, nous avons pu vérifier les corrélations entre les sous-échelles respectives du CSI et du Kidcope. Les plus fortes corrélations ont été obtenues entre sept des huit sous-échelles prévues au CSI et les énoncés au Kidcope y correspondant (étendue $=0,33$ à 0,77 ) . 
Les relations entre les scores factoriels obtenus dans le ACOPE et dans le Kidcope ont également été examinées. La plupart des corrélations les plus élevées entre les différentes échelles ont été obtenues entre les scores factoriels au ACOPE et les énoncés au Kidcope. Cependant, les corrélations n'étaient pas aussi importantes que celles retrouvées pour le $C S I$ (étendue $=0,08$ à 0,62 ). Ce résultat était prévisible puisque les scores factoriels au ACOPE sont plus spécifiques et moins concordants avec ceux choisis pour le Kidcope.

Le ACOPE permet d'identifier les stratégies d'adaptation habituellement utilisées par les adolescents, alors que le Kidcope et le CSI demandent aux adolescents de nommer la stratégie utilisée lors d'un événement particulier ayant eu lieu dernièrement. Ainsi, si on présume qu'une mesure évaluant un trait général de l'adaptation ne devrait corréler que modérément avec des stratégies spécifiques d'adaptation utilisées dans une situation particulière (Lazarus et Folkman, 1984), les résultats des études de validité vérifiant la relation entre le Kidcope, le CSI et le $A C O P E$ restent cohérents avec cette théorie et s'orientent dans la direction prévue.

Nous avons également examiné la relation entre la fréquence d'utilisation de chacune des stratégies d'adaptation et l'évaluation de leur efficacité, c'est-à-dire à quel point cette stratégie d'adaptation s'est avérée utile pour gérer ce stresseur? Vraisemblablement, les stratégies d'adaptation perçues comme étant les plus utiles seront celles le plus fréquemment utilisées. Dans un échantillon de 609 adolescents, à une question du Kidcope portant sur un stresseur personnel, la corrélation entre la fréquence et l'efficacité des stratégies d'adaptation révèle que les corrélations les plus fortes sont obtenues entre la fréquence et l'efficacité de chacune des stratégies (Spirito et al., 1988). Notons ici que le Kidcope est une des rares mesures qui examine à la fois la fréquence et l'efficacité d'une stratégie d'adaptation. Le format des réponses de cet instrument permet d'examiner non seulement le nombre de stratégies utilisées mais aussi leur efficacité respective dans la prédiction de la trajectoire du développement.

\section{LES DONNÉES NORMATIVES DU KIDCOPE: LES DIFFÉRENCES QUANT À L'ÂGE, AU SEXE ET AUX STRESSEURS}

Dans une des premières études menées auprès d'adolescents de 14 à 17 ans (Stark, Spirito, Williams et Guevremont, 1989), l'utilisation des stratégies d'adaptation ne se distinguait pas selon l'âge. Comme le proposent 
Curry et Russ (1985), ce résultat s'explique peut-être par de plus grandes différences dans l'adaptation chez les plus jeunes enfants que chez ceux parvenus au milieu de l'adolescence.

Notre étude avec de jeunes enfants (Spirito et al., 1991) a démontré quelques différences significatives dans l'utilisation des stratégies d'adaptation selon le sexe. Face à un problème général (se faire gronder par ses parents), les garçons avaient plus tendance à blâmer les autres, tandis que les filles utilisaient la résolution de problème. Curry et Russ (1985), quant à eux, n'ont pas trouvé de différences liées au sexe dans leur échantillon de jeunes enfants, alors que pour Stark et ses collaborateurs (1989), l'utilisation des stratégies d'adaptation se distingue en fonction du sexe à l'adolescence. En effet, les jeunes filles rapportent avoir recours plus souvent au soutien social que les garçons. Ceux-ci font plus usage de la pensée magique que les jeunes filles et considèrent, plus que les filles, que la résignation est efficace. Les différences sexuelles dans l'utilisation des stratégies d'adaptation ne sont pas dues aux différents problèmes choisis et définis par les filles et les garçons. En fait, les résultats démontrant que les adolescentes utilisent plus fréquemment la régulation émotionnelle (lorsqu'il s'agit d'un problème impliquant un ami ou une amie) et le soutien social comme stratégies d'adaptation concordent avec les résultats d'autres études menées auprès d'adultes (Billings et Moos, 1981; Stone et Neale, 1984). Ainsi, il semble que, dès le milieu de l'adolescence, les filles sont plus expressives et recherchent plus de soutien social que les garçons lorsqu'elles doivent gérer des situations stressantes. À partir de ces résultats, on peut dire que les différences selon le sexe ne semblent pas différencier les méthodes d'adaptation des jeunes enfants. Cependant, le fait que les réponses des adolescents plus vieux au Kidcode diffèrent significativement suivant le sexe souligne les changements dans le développement.

Dans notre étude, les stratégies d'adaptation appliquées par les jeunes enfants diffèrent selon le stresseur qu'ils rapportent (Spirito et al., 1991). La stratégie de blâmer les autres se retrouve souvent lorsqu'il s'agit de problèmes impliquant des amis et la fratrie. La régulation émotionnelle est davantage rapportée face à des problèmes touchant la fratrie. Ce résultat démontrant des différences dans l'utilisation des stratégies d'adaptation pour gérer les problèmes au sein de la fratrie est logique. En effet, la tendance des enfants, à exprimer leurs émotions, à attribuer le blâme à l'autre, à se résigner face aux problèmes impliquant la fratrie peut être admise par tous les parents et les thérapeutes d'enfants, et cela, en dépit du manque de soutien empirique. En général, il semble que les adolescents font usage d'une plus grande variété de stratégies lorsqu'ils tentent de gérer des 
problèmes touchant les relations garçons/filles que lorsqu'il s'agit de problèmes liés à l'école ou aux parents (Stark et al., 1989). L'emploi plus fréquent de diverses stratégies d'adaptation lors de problèmes impliquant l'amoureux ou l'amoureuse suggère un effort plus organisé de la personne pour en arriver à la résolution de ce problème. Par contre, l'utilisation de différentes stratégies d'adaptation est contradictoire avec les résultats de Wills (1986). En effet, en évaluant un échantillon d'élèves de septième ou huitième année, donc plus jeunes, Wills rapporte une forte stabilité dans le choix des stratégies d'adaptation utilisées pour les problèmes impliquant l'école, les parents, la santé, la tristesse et les problèmes entre amis. Il est important de noter ici que les problèmes avec les amoureux ou les amoureuses n'étaient pas nécessairement évalués dans l'étude de Wills (1986). Notre étude a été la première dans le domaine à montrer une augmentation du nombre de stratégies. Par ailleurs, ces différences peuvent être liées à la situation problématique dans laquelle les personnes se trouvent.

\section{LES RÉSULTATS DU KIDCOPE : ÉCHANTILLONS CLINIQUES}

L'étape suivante du développement du Kidcope a porté sur son utilisation dans des populations cliniques. D'abord, nous avons examiné les types de problèmes et les stratégies d'adaptation rapportés par les adolescents qui tentent de se suicider, comparativement à ceux d'adolescents non suicidaires (Spirito, Stark et Overholser, 1989). La façon dont les personnes s'adaptaient aux facteurs associés aux tentatives de suicide était comparée aux problèmes rapportés par des groupes appariés d'adolescents " normaux ", en détresse et pas en détresse. Puisque les ouvrages récents démontrent que les adolescents qui tentent de se suicider possèdent de faibles habiletés dans la résolution de problèmes, nous avons émis l'hypothèse que les jeunes ayant des tendances suicidaires utiliseraient moins fréquemment que les adolescents non suicidaires des stratégies d'adaptation actives (résolution de problèmes, soutien social) et qu'ils considéreraient ces stratégies comme moins aidantes.

Nous avons trouvé des ressemblances étonnantes dans l'utilisation des stratégies d'adaptation rapportée par des adolescents suicidaires et celle des adolescents non suicidaires. Les seules différences entre ces deux groupes concernent le retrait social et la pensée magique. Plus précisément, les adolescents suicidaires ont utilisé le retrait social plus fréquemment que les adolescents "normaux", en détresse et sans détresse. Par ailleurs, les adolescents suicidaires et en détresse ont recours plus souvent à la pensée magique que les adolescents qui ne sont pas en détresse. Toutefois, les groupes ne se distinguent pas quant à leur utilisation de la 
distraction, de la restructuration cognitive, de l'autocritique, du fait de blâmer les autres, de la régulation émotionnelle et du soutien social. Ces résultats suggèrent que le retrait social serait une stratégie d'adaptation particulièrement inadaptée et qu'il pourrait augmenter la probabilité des tentatives de suicide. Ainsi, il semble que les adolescents suicidaires utilisent une variété de stratégies d'adaptation et qu'ils font face à leurs problèmes d'une manière similaire aux adolescents non suicidaires, à l'exception toutefois que les adolescents suicidaires auraient parfois tendance, à un certain moment, à se retirer des autres, ce que les adolescents non suicidaires ne feraient pas. De plus, les adolescents en détresse et suicidaires peuvent se tourner davantage vers la pensée magique, omettant alors d'utiliser des stratégies de résolution de problème.

Par la suite, nous avons élargi notre examen clinique en étudiant, à partir du Kidcope, les stratégies d'adaptation utilisées par trois groupes d'adolescents hospitalisés en psychiatrie (des adolescents ayant fait des tentatives de suicide, des adolescents ayant des idées suicidaires et des adolescents dépressifs non suicidaires) et des groupes contrôles " normaux» (Spirito, Francis, Overholser et Frank, 1996). Puisqu'il est difficile d'évaluer les stratégies d'adaptation en dehors du contexte qui les suscite, nous avons imaginé un scénario standard pour contrôler les différents types de problèmes retrouvés chez les participants. Comme dans l'étude précédente, nous partions de l'hypothèse que les adolescents ayant fait des tentatives de suicide utiliseraient plus de stratégies d'évitement que les adolescents hospitalisés en psychiatrie, ceux ayant des idées suicidaires, les dépressifs et le groupe contrôle. Nous avons de plus évalué l'effet de l'humeur dépressive sur l'adaptation des patients hospitalisés en psychiatrie. À partir de la documentation limitée à ce momentlà, l'hypothèse voulait que, dans l'ensemble, l'humeur dépressive soit associée à plus de stratégies d'évitement. Finalement, nous avons évalué la relation entre la tristesse liée au scénario hypothétique et l'utilisation des stratégies d'adaptation. Nous avions prévu que les adolescents rapportant de la tristesse utiliseraient plus de stratégies d'évitement et d'expression émotionnelle que les adolescents n'éprouvant pas de tristesse, et auraient recours à des stratégies d'adaptation moins actives que celles utilisées par ces derniers.

Tout comme dans l'étude clinique précédente, l'isolement social a été utilisé plus souvent par les adolescents hospitalisés en psychiatrie ayant fait des tentatives de suicide. Cependant, tous les participant hospitalisés en psychiatrie (ceux ayant fait des tentatives, ceux ayant des idéations suicidaires, les dépressifs et les non suicidaires) ont plus utilisé l'isolement social que les groupes contrôles et les personnes ayant tenté 
de se suicider et hospitalisés pour des raisons médicales. Ainsi, l'isolement social n'est pas spécifique aux personnes ayant tenté de se suicider mais plutôt caractéristique d'un déséquilibre émotionnel général: plus la psychopathologie est sévère, plus l'isolement social est évident.

De plus, les participants dépressifs ont utilisé la restructuration cognitive moins souvent que les participants non dépressifs. Des résultats similaires ont été trouvés pour les participants chez qui le scénario hypothétique avait provoqué de la tristesse. Les participants dépressifs utilisaient moins souvent le soutien social, mais plus souvent l'autocritique et le retrait social comparativement aux participants non dépressifs. Ces résultats vont dans le même sens que ceux de Wierzbicki et Carver (1989), lesquels ont démontré une diminution de l'adaptation cognitive lorsque la dépression augmente. Ebata et Moos (1991) ont également démontré qu'une grande détresse entraîne moins de résolution de problème et plus d'évitement cognitif. Par conséquent, les recherches futures qui examineront l'adaptation des adolescents dépressifs sur un continuum (un échantillon de la population ne recevant pas de traitement, des patients hospitalisés et des patients en clinique externe) pourraient être très pertinentes pour une meilleure compréhension des résultats. Il ressort néanmoins de nos résultats que les adolescents dépressifs sont un groupe auprès duquel il est très important $\mathrm{d}$ 'intervenir eu égard aux stratégies d'adaptation.

\section{L'ADAPTATION DES ENFANTS FACE À UNE MALADIE CHRONIQUE}

Après les études avec le Kidcope auprès d'enfants en santé et d'adolescents (1989), nous avons entrepris une étude transversale sur l'utilisation et l'efficacité des stratégies d'adaptation chez des enfants atteints d'une maladie chronique. Ainsi, des enfants et des adolescents atteints de maladie chronique ou d'autres maladies sévères ont été rencontrés en entrevue pendant leur séjour à l'hôpital (Spirito, Stark et Tyc, 1996). Nous avons obtenu un franc succès dans le recrutement de ces familles en partie parce que nous expliquions que cette étude permettrait d'identifier les processus adaptatifs utilisés par les enfants pour gérer la maladie et l'hospitalisation. Les patients du groupe d'enfants atteints de maladie chronique souffrent de cancer, diabète, asthme, drépanocytose (sickle cell anemia) et fibrose kystique. Nous avons demandé à ces enfants de décrire un problème lié à la santé auquel ils ont dû faire face dans les mois précédents, puis évaluer le degré d'anxiété, de tristesse et de colère que ce problème avait suscité. 
Enfin, ils ont répondu au Kidcope en indiquant la fréquence avec laquelle ils avaient utilisé les diverses stratégies d'adaptation pour gérer le problème décrit plus tôt et l'efficacité de ces stratégies pour soulager leur détresse. Les patients dans le groupe des malades souffrant de maladies sévères étaient admis à l'hôpital pour des chirurgies orthopédiques, des appendicites, des douleurs abdominales et autres problèmes sévères. Ces patients nous ont eux aussi décrit un problème touchant leur hospitalisation et ont par la suite répondu au Kidcope en gardant toujours en tête ce même problème. Ces stresseurs ont été regroupés suivant trois aspects différents : a) un aspect spécifique lié à l'hospitalisation (difficulté à dormir, mauvaise nourriture, manque d'intimité), b) un problème touchant leur maladie (une préoccupation liée à leur maladie et provoquée par l'hospitalisation), ou c) une inquiétude liée à la douleur (être incommodé à cause de la maladie ou d'une procédure médicale). Les enfants des deux groupes devaient ensuite nous décrire un problème quotidien survenu dans les derniers mois puis répondre au Kidcope en fonction de ce problème. Cela nous a permis d'examiner en quoi les stratégies d'adaptation face aux problèmes " médicaux " se distinguaient de celles utilisées face à des problèmes liés à la vie quotidienne.

Des résultats significatifs ont été obtenus en fonction du stade développemental (enfants/adolescents) et de la nature de la maladie (sévère versus chronique). Dans notre étude, les adolescents utilisaient plus les stratégies cognitives (résolution de problème et résignation) que les enfants. Presque tous les enfants avaient recours à la pensée magique, comparativement aux trois quarts des adolescents. Ces résultats vont dans le même sens que la documentation qui démontre que les enfants utilisent plus de stratégies cognitives en vieillissant (Brown, O'Keeffe, Sanders et Baker, 1986). Bien que souvent considérées comme étant des stratégies inadaptées (pensée magique), la prédominance de ces stratégies chez les enfants suggère une qualité adaptative qu'il serait certainement intéressant d'examiner dans des recherches futures. Toutefois, on ne trouve aucun effet attribuable au sexe, contrairement aux autres résultats de nos travaux utilisant le Kidcope (Stark et al., 1989) auprès d'adolescents. Il est possible que le sexe des individus soit un facteur moins important à considérer quand on doit évaluer les stratégies adaptatives adoptées face à un stresseur lié à l'hospitalisation que lorsqu'il s'agit d'un problème lié à la vie quotidienne.

Plutôt que d'employer des stratégies d'adaptation plus actives, les enfants atteints de maladie chronique ont tendance à utiliser des stratégies d'évitement et, vraisemblablement, moins de stratégies adaptatives du genre distraction et pensée magique et de stratégies négatives (autocritique). Selon nos connaissances, cette étude a été la première à distinguer 
des différences dans l'utilisation de stratégies d'adaptation en fonction de la nature de la maladie. Il y avait également un effet d'interaction entre l'âge et la maladie pour quelques stratégies: le retrait social, l'autocritique et la pensée magique. Les jeunes enfants blessés ou atteints d'une maladie sévère ont plus utilisé le retrait social que les adolescents dans les mêmes conditions de santé. Par contre, les adolescents souffrant d'une maladie chronique ont utilisé l'autocritique moins souvent que les adolescents blessés ou atteints d'une maladie sévère. Cette constatation peut s'expliquer par le fait que la plupart des adolescents avaient un rôle à jouer dans la blessure ou la maladie dont ils souffraient; leurs comportements pouvaient avoir causé leurs blessures. De plus, les différences observées entre le deux groupes pourraient être liées à la fréquence d'hospitalisation. En fait, la plupart des jeunes ayant une maladie sévère ou une blessure vivent leur première hospitalisation, alors que ceux atteints d'une maladie chronique ont déjà vécu plusieurs hospitalisations. Une proportion des plus jeunes enfants atteints de maladie chronique (19\%) ont eu recours à l'autocritique pour faire face au problème associé à leur hospitalisation. Ce résultat suggère une tendance, chez certains jeunes enfants, à se blâmer pour certains aspects de leur maladie chronique ou encore pour leur hospitalisation. Par conséquent, les cliniciens devraient vérifier sur une base régulière le degré de culpabilité que l'enfant entretient face à sa situation actuelle.

La durée de l'hospitalisation n'était pas liée aux problèmes choisis concernant l'hôpital, la quantité de détresse ressentie ou les styles de stratégies d'adaptation utilisées par les deux groupes (maladie chronique ou sévère). Bien que toutes les expériences liées à l'hôpital soient différentes, elles n'ont pas eu d'effet marquant sur l'utilisation des stratégies d'adaptation. Néanmoins, on considère que l'utilisation des stratégies d'adaptation peut être liée au temps écoulé depuis l'annonce du diagnostic, au nombre de procédures médicales précédentes (Smith, Ackerson, Blotcky et Berkow, 1990) et aux mesures de chronicité de la maladie. Donc, les changements de stratégies d'adaptation sont peut-être plus apparents lorsqu'on examine l'ajustement à des phases variées du traitement durant les hospitalisations. En fait, il est possible que l'adaptation soit un processus tellement graduel et dynamique qu'il soit nécessaire d'en faire une évaluation longitudinale pour déceler les effets du temps.

Nous avons fait l'hypothèse que des problèmes liés à la douleur appelleraient des stratégies plus actives que les problèmes liés à la maladie. Cette hypothèse se base sur la présomption que les enfants ont plus de contrôle sur la douleur que sur les problèmes touchant la maladie. Cette hypothèse a été confirmée probablement parce que les enfants ont plus de contrôle sur les procédures liées à la douleur que sur les stresseurs associés 
à la maladie (Suls et Fletcher, 1985). La détresse suscitée par les problèmes liés à l'hôpital a été associée plus fréquemment à certaines stratégies d'adaptation. Ainsi, plus le niveau d'anxiété est élevé, plus les stratégies adaptatives actives sont utilisées (en particulier la résolution de problème). La stratégie évitante a également été plus fréquemment employée par les enfants anxieux. En fait, la plupart des études démontrent que les enfants anxieux utilisent davantage de stratégies d'adaptation négatives telles que la dramatisation (Brown et al., 1986). Dans notre étude, l'anxiété était considérée en fonction d'un stresseur spécifique et non pas comme une caractéristique individuelle, ce qui explique peut-être que les stratégies d'adaptation actives puissent se révéler aidantes. Il est possible que les enfants aient tenté de s'adapter à un stresseur spécifique provoqué par l'hospitalisation en recherchant de l'information et en parlant avec les autres. Ainsi, les enfants qui se disaient anxieux ont utilisé un plus grand nombre de stratégies d'adaptation que les enfants non anxieux. Ce résultat s'ajoute à d'autres qui proposent que le nombre de stratégies d'adaptation utilisées s'accroît en fonction des exigences stressantes qui exercent une pression sur l'individu (Curry et Russ, 1985).

Les stratégies d'adaptation actives telles que le soutien social et la pensée magique ont été davantage utilisées par les enfants qui se disaient tristes par suite de l'avènement du stresseur. Dans la plupart des cas, les enfants et les adolescents n'avaient pas beaucoup de contrôle sur les stresseurs identifiés; par conséquent, ils peuvent s'être tournés vers des stratégies cognitives (pensée magique) et comportementales (soutien social) pour diminuer leur détresse. Le résultat indiquant la recherche de soutien social démontre toute l'importance de la présence des parents et d'un environnement chaleureux (un personnel attentif aux besoins des enfants pendant l'hospitalisation) surtout pour les enfants qui se sentent tristes.

Les résultats indiquant la colère sont intéressants à relever ici. Tout comme pour la tristesse, la pensée magique a été fortement utilisée lorsque les problèmes liés à l'hôpital suscitaient de la colère chez les patients. Ce résultat pourrait également être associé au manque de contrôle ou au manque d'habileté des enfants à exprimer la colère de manière appropriée durant $l^{\prime}$ hospitalisation à cause de la confiance qu'ils portent au personnel infirmier et médical. De plus, le retrait social et l'autocritique sont inversement proportionnels à l'expression de la colère. Ainsi, plus les patients sont fâchés, moins ils se retirent socialement et moins ils se blâment pour leurs problèmes. Ce résultat confirme ceux des recherches portant sur des adultes et voulant que la colère puisse avoir une valeur adaptative lorsque les patients se remettent de leur maladie ou de leur hospitalisation. 


\section{IMPLICATIONS CLINIQUES}

Les résultats de notre étude auprès d'enfants et d'adolescents hospitalisés comportent plusieurs implications pertinentes pour le travail clinique. Premièrement, bien que les chercheurs portent leur attention sur l'adaptation face à des procédures douloureuses, d'autres stresseurs associés à l'hospitalisation revêtent une aussi grande importance et méritent d'être considérés par les cliniciens. Deuxièmement, même si les enfants atteints de maladie chronique et les enfants blessés ou souffrant de maladie sévère décrivent les mêmes problèmes, des différences significatives démontrent qu'ils ne s'y adaptent pas de la même façon. Ces différences peuvent refléter la quantité de détresse provoquée par ces problèmes et être dues à la différence dans le degré de fréquentation du milieu hospitalier. Par ailleurs, il est important de faire preuve de rigueur lorsque nous appliquons aux enfants atteints de maladie chronique les résultats de recherche obtenus auprès des enfants souffrant d'une maladie sévère/être blessé.

Les différences adaptatives mises en lumière par la mesure de dépistage indiquent que des recherches plus poussées méritent d'être réalisées. Cette découverte démontre également l'utilité clinique de cette mesure pour le travail quotidien des psychologues évaluant des enfants dans un contexte hospitalier. Il serait également profitable de rassembler plus $\mathrm{d}^{\prime}$ informations normatives sur les stratégies d'adaptation que les enfants utilisent spontanément lors de leurs hospitalisations. Cette étude a été réalisée à partir d'un échantillon très hétérogène de participants atteints de maladie chronique, ce qui limite l'élaboration de conclusions portant sur des sous-groupes d'enfants atteints de maladie chronique. Cependant, plusieurs croient qu'une approche non catégorielle (Stein et Jessop, 1982) est plus appropriée à l'étude de l'ajustement des enfants souffrant de maladie chronique. Dans cette perspective, on suppose que certaines ressemblances entre les maladies sont plus importantes dans la compréhension du fonctionnement individuel que ne le sont les questions spécifiques liées à une maladie.

\section{DIFFÉRENCES DE L'ADAPTATION SELON LA SITUATION}

Nous nous sommes intéressés à l'utilité des évaluations situationnelles de l'adaptation dans des cas de maladie chronique. Pour ce faire, les sujets devaient répondre au Kidcope en fonction d'un problème quotidien et d'un problème lié à la maladie (Spirito, Stark, Gil et Tyc, 1995). Nous avons mené plusieurs analyses pour déterminer si l'adaptation était stable et plus fortement affectée par des prédispositions individuelles, ou si elle 
avait tendance à varier selon la situation. $\mathrm{D}^{\prime}$ abord, nous avons regardé si les variables modératrices (âge, sexe) déterminaient de manière différente les stratégies d'adaptation utilisées pour les problèmes liés à la maladie chronique et les problèmes quotidiens. Nous avons formulé l'hypothèse que si l'adaptation était plus fortement touchée par des prédispositions individuelles (trait) que par la situation, les effets du sexe et de l'âge devraient être similaires pour le problème lié à la vie quotidienne et le problème lié à la maladie chronique.

Les résultats touchant l'âge suggèrent que l'adaptation varie en effet selon la situation. Le seul effet d'âge convergeant avec les deux types de problèmes (quotidien / maladie chronique) suggère que les adolescents utilisent la résignation comme stratégie d'adaptation plus souvent que les jeunes enfants. En fait, les enfants plus vieux avaient tendance à blâmer les autres plus souvent que les plus jeunes lorsqu'il s'agissait d'un problème lié à la maladie chronique. Toutefois, le résultat était inversé pour le problème quotidien; les plus jeunes enfants blâmaient les autres plus souvent que les plus vieux. Le résultat touchant les enfants plus vieux et leur utilisation plus fréquente de la résignation peut être expliqué par le fait que les adolescents ont atteint le stade des opérations formelles et qu'ils ont donc une compréhension plus développée de ce qu'ils vivent. Ils comprennent ainsi que les problèmes qu'ils vivent sont irréversibles et qu'aucune solution ne viendra les contrer (Schowalter, 1983). Ce résultat peut aussi être associé au fait que les adolescents vivent leur maladie depuis plus longtemps que les jeunes enfants. Ce résultat est similaire à une étude qui démontre que le sentiment d'appartenance à un groupe de malades est plus fréquent chez des enfants plus âgés, qui souffrent de maladie affectant l'hémoglobine (sickle cell disease), que chez des enfants plus jeunes (Gil, Williams, Thompson et Kinney, 1991).

D'un autre côté, le sexe des enfants influence l'emploi de stratégies d'adaptation pour les problèmes de maladie chronique, mais pas pour les problèmes liés à la vie quotidienne. Ainsi, face à des problèmes liés à la maladie chronique, les garçons utilisent la restructuration cognitive et l'autocritique plus souvent que les filles qui, elles, utilisent plus de régulation émotionnelle et de soutien social que les garçons. Par contre, lorsqu'il s'agit d'un problème lié à la vie quotidienne, les garçons et les filles ont appliqué ces stratégies de manière équivalente. Ces résultats démontrent que le sexe influence les stratégies d'adaptation employées face à des problèmes touchant la maladie, et soulèvent l'hypothèse de la tolérance au stress. En fait, une exposition répétitive aux stresseurs liés à la maladie et la quantité de stress suscité amènent les garçons et les filles à adopter des stratégies qualitativement différentes pour gérer l'augmentation du stress. Le soutien social et les autres ressources peuvent 
être plus facilement disponibles pour les filles lorsqu'elles subissent un stress élevé; elles utilisent en effet la régulation émotionnelle et le soutien social afin de mieux s'adapter. De leur côté, les garçons choisissent des mécanismes d'adaptation différents tels que la restructuration cognitive et l'autocritique. L'hypothèse de la tolérance au stress soutient également que l'adaptation se fait sur un continuum et est dépendante d'un stresseur (Compas, 1987). Ce résultat est intéressant; il doit être confirmé et exploré dans le futur.

Nous avons ensuite utilisé les données recueillies pour examiner la variabilité situationnelle de l'adaptation en analysant les changements observés dans l'adaptation en fonction du type de problème lié à la maladie (douleur/maladie) et de l'humeur. Plusieurs stratégies ont été utilisées pour les deux types de problèmes, mais quelques-unes diffèrent, telles que la distraction, la régulation émotionnelle et le blâme placé sur les autres. L'humeur n'a qu'un faible effet sur l'utilisation des stratégies d'adaptation pour les problèmes liés à la maladie chronique; seule la restructuration cognitive variait selon le degré d'anxiété. Contrairement aux résultats décrits plus tôt pour l'âge et le sexe, ces résultats soutiennent l'idée de la stabilité de l'adaptation plutôt que celle de sa variabilité suivant la situation. Les différences situationnelles selon le type de stresseurs généraux (maladie chronique / problèmes quotidiens) sont plus marquées pour l'adaptation que celles plus subtiles entre les catégories de stresseurs (stress lié à la maladie / stress lié à la douleur).

Le troisième test évaluant comment l'adaptation varie suivant la situation s'est révélé le plus significatif. Chaque enfant et chaque adolescent devaient décrire comment ils s'adaptent à un problème lié à la santé et à un autre tracas lié au quotidien. Les analyses examinant la stabilité des stratégies d'adaptation entre ces deux situations ont révélé des patterns modérément cohérents. Ces résultats soutiennent la notion que l'adaptation est un processus qui diffère quelque peu chez les individus selon la situation à laquelle ils font face, et qui présente tout de même une légère stabilité (Lazarus et Folkman, 1984). Bull et Drotar (1991) ont mené une étude auprès d'enfants en rémission d'un cancer et ayant complété leur traitement. Ils ont trouvé des différences dans l'utilisation des stratégies d'adaptation face à un stresseur lié au cancer et à un stresseur qui n'y était pas relié. Ces résultats suggèrent un peu plus de variabilité suivant la situation que chez notre échantillon d'enfants qui sont toujours en traitement actif. 


\section{IMPLICATIONS CLINIQUES}

Les résultats de notre étude proposent que, malgré une certaine stabilité, des différences existent entre les situations ayant trait à la maladie chronique et celles ayant trait au quotidien. Par conséquent, lorsque les cliniciens veulent faciliter l'ajustement des enfants à leur maladie, ils devraient évaluer l'adaptation des enfants face à un problème lié à la maladie plutôt que devant un problème quotidien. Cette pratique semble plus pertinente dans la mesure où elle guide les recommandations préventives adressées aux parents et aux professionnels de la santé.

\section{STRATÉGIES SIMPLES D’ADAPTATION OU PATTERNS DE STRATÉGIES D'ADAPTATION}

Un autre domaine suscite notre intérêt et soulève la question suivante: Est-ce que l'adaptation est mieux comprise lorsqu'on l'aborde sous l'angle d'une stratégie simple d'adaptation ou en tant que patrons de stratégies? Donaldson, Prinstein, Danovsky et Spirito (2000) ont comparé le pattern des scores obtenus au Kidcope par les enfants hospitalisés et atteints de maladie sévère ou chronique, qui rapportaient un stresseur lié à la maladie, à celui de 700 enfants en santé, dont le stresseur était lié à l'école, aux parents et à un problème interpersonnel. Même si on retrouvait des différences dans l'utilisation individuelle des stratégies d'adaptation, on peut relever une cohérence interne considérable dans le patron de fréquence des stratégies d'adaptation pour les quatre catégories de stresseurs. Les corrélations intra-groupes ont été calculées dans le but de vérifier la relation entre les dix stratégies d'adaptation au Kidcope dans chacune des paires de stresseurs. Les corrélations variaient de 0,77 à $0,97(r$ moyen $=, 89)$, ce qui suggère que des profils similaires d'adaptation existent pour les quatre types de stresseurs. En fait, la corrélation intra-groupe qui considérait simultanément les quatre types de stresseurs obtient un coefficient de 0,89. Les enfants utilisent davantage la pensée magique que toute autre stratégie pour les quatre catégories de stresseurs. Puis, viennent la régulation émotionnelle et la résolution de problème. Tous les enfants utilisent moins souvent l'autocritique, le blâme attribué aux autres et la résignation.

\section{IMPLICATIONS CLINIQUES}

L'exploration des patrons d'adaptation semble procurer une tout autre forme d'information que celle obtenue avec les stratégies individuelles d'adaptation. Les prochaines études sur l'adaptation des enfants, en particulier 
celles s'intéressant aux conséquences sur le développement, pourraient inclure de l'information portant sur les stratégies d'adaptation individuelles et sur les patrons de stratégies utilisés en réponse à différents types de stresseurs. La séquence d'utilisation de ces stratégies est également importante à considérer et peut devenir un indicateur pertinent pour observer les changements positifs survenus après une intervention.

\section{ÉVALUATION ACTUELLE DE L'ADAPTATION DANS DES POPULATIONS PÉDIATRIQUES}

Malheureusement, il n'existe pas de consensus chez les chercheurs quant à une mesure critère (gold standard) permettant d'évaluer l'adaptation en psychologie pédiatrique. L'absence d'une telle mesure est en partie attribuable à l'existence de plusieurs définitions et conceptions de l'étude de l'adaptation. Même si deux études évaluent la même stratégie ou le même style d'adaptation, les énoncés permettant de les mesurer sont bien souvent très différents. Le manque de consistance dans l'application de cette mesure d'adaptation n'est pas surprenant puisqu'il est improbable qu'une seule mesure de coping puisse convenir à toutes les situations.

Un intérêt s'est développé pour l'analyse des données au Kidcope en fonction d'une structure factorielle (higher order factor structure) (adaptation positive/négative, adaptation selon l'approche/l'évitement) plutôt que des stratégies d'adaptation individuelles. De tels scores factoriels sont particulièrement utiles dans les analyses de régression multiple afin de vérifier la contribution de l'adaptation parmi d'autres variables attribuables à l'ajustement global. Nous avons amassé plus de 3000 Kidcope, ce qui nous a permis d'examiner la structure factorielle de la mesure en utilisant: a) tout l'échantillon, b) une partie de l'échantillon, c) des sousgroupes précis (adolescents versus enfants, garçons/filles) ou encore d) plusieurs situations spécifiques (problèmes liés à la maladie, conflits parents/ enfant). Par exemple, nous avons recueilli 600 Kidcope remplis par des adolescents qui devaient expliquer comment ils réagiraient face à un ami suicidaire. En utilisant tout l'échantillon, nous avons fait ressortir une structure factorielle unidimensionnelle composée de cinq énoncés. À partir d'analyses séparées pour le sexe, les résultats de l'échantillon des filles indiquaient la même structure à cinq énoncés, au même titre que les garçons qui ont démontré une structure factorielle unidimensionnelle identique, exception faite d'un énoncé additionnel (Spirito, 1996). Nous avons également choisi un sous-échantillon d'enfants souffrant de maladie chronique et avons exécuté des analyses factorielles pour différents 
problèmes choisis par les enfants (hospitalisation, procédures médicales, inquiétudes par rapport à la progression de la maladie). Selon le type de situation, on obtient une solution factorielle à deux facteurs ou à un seul. Par exemple, une structure à deux facteurs (adaptation selon l'approche/ l'évitement) apparaissait lorsque le problème choisi impliquait des procédures médicales aversives, alors qu'une structure factorielle unidimensionnelle ressortait lorsque le stresseur consistait en une période d'hospitalisation prolongée.

Le changement dans la structure factorielle ne devrait pas nous étonner. En fait, la catégorisation d'une stratégie d'adaptation ne peut être indépendante de la situation. La fonction ou la classification d'une stratégie d'adaptation (adaptative/inadaptée) n'est pas la même dans toutes les situations. Coyne et Gottlieb (1996) s'opposent à l'élaboration d'une mesure d'adaptation auto-rapportée fortement standardisée et encouragent plutôt les chercheurs à considérer d'autres moyens pour évaluer entièrement l'adaptation. Ils suggèrent les entrevues semi-structurées, des journaux intimes et des listes visant à tester les hypothèses d'un projet de recherche spécifique, plutôt que d'utiliser des mesures imparfaites mais tout de même publiées. De telles mesures pourraient néanmoins faciliter la diminution des données des chercheurs avant les analyses statistiques et aider les cliniciens à comprendre globalement l'approche généralement adoptée par un enfant qui doit s'adapter.

Existe-t-il des solutions à ce dilemme entre, d'un côté, des structures factorielles variées suivant les situations et, de l'autre, des dimensions globales de l'adaptation que voudraient trouver les chercheurs? Plusieurs approches peuvent être considérées. D'abord, on peut développer des facteurs spécifiques pour des données particulières. Toutefois, les études doivent porter sur une population suffisamment grande (150 à 200 sujets) pour compléter des analyses factorielles à partir des données recueillies dans un projet spécifique. Le nombre de sujets pose souvent un problème en psychologie pédiatrique puisque le nombre de sujets disponibles, spécialement pour les études traitant l'intervention, peut être plus petit que le requièrent les analyses factorielles. Ensuite, on peut regrouper des experts devant décrire la situation dans laquelle l'adaptation est présentée aux enfants et les laisser décider quelles stratégies devraient être regroupées comme adaptation positive/négative, adaptation selon l'approche/l'évitement, etc., selon le modèle conceptuel visé. Cependant, cette approche n'est pas nécessairement très valide du point de vue empirique.

Dans le cas du Kidcope, comme dans celui des autres mesures de dépistage qui présentent un nombre limité d'énoncés, une troisième solution serait d'observer chaque énoncé ou chaque sous-échelle. Bien que la 
concision des échelles du Kidcope soit intéressante, une grande partie des informations importantes est perdue par l'utilisation de telles mesures de dépistage. Ainsi, l'analyse des données énoncé par énoncé aide à faire une meilleure exploration des complexités du processus d'adaptation. Plusieurs énoncés de l'échelle qui se révèlent plus pertinents à la situation pourraient être choisis a priori pour des analyses de régression. En examinant un énoncé à la fois, c'est-à-dire une stratégie d'adaptation spécifique, il est particulièrement important de regrouper les données de validité test-retest pour justifier l'analyse menée pour chaque énoncé pris individuellement. Idéalement, cela devrait être fait séparément pour chaque étude. Cependant, cette démarche nécessite beaucoup de temps et augmente la complexité de la recherche.

Une quatrième solution consiste à utiliser des instruments de mesure avec plusieurs items portant sur la même stratégie adaptative afin de déterminer les principales dimensions ou échelles. Le Self-Reported Coping Survey (Causey et Dubow, 1992) est une échelle composée de 34 énoncés à partir desquels sont formées deux sous-échelles (recherche de soutien social et résolution de problème) étiquetées "Approche " et trois sous-échelles (mise à distance, internalisation et externalisation) nommées “Évitement». La valeur positive ou négative de ces souséchelles (positif/négatif) peut changer selon les stresseurs examinés dans une recherche donnée. Ainsi, le fait qu'une stratégie soit classée dans évitement ne signifie pas toujours qu'elle soit inadaptée, non plus qu'une autre classée dans approche soit nécessairement appropriée dans une situation particulière.

Finalement, lorsque l'adaptation est la variable centrale à l'étude, l'entrevue demeure un très bon moyen d'évaluation. Une entrevue poussée avec l'enfant sur ses stratégies d'adaptation favorise souvent une meilleure compréhension de la nature du processus d'adaptation que les mesures auto-rapportées (telles que les mesures de dépistage). Les entrevues découlent d'un modèle théorique (surveillance/être brusqué (monitoring/blunting)l ; Miller, Sherman, Combs et Kruus, 1992; primaire/secondaire/abandon des stratégies de contrôle; Band, 1990). Par conséquent, des scores sommaires pour des construits spécifiques peuvent habituellement en découler, ce que la plupart des chercheurs trouvent utile. Les entrevues augmentent considérablement le temps nécessaire pour la cueillette de données, ce qui est souvent une raison suffisante pour ne pas les utiliser. Faire passer aux mêmes sujets une mesure de dépistage auto-rapportée et une entrevue sur les deux ou trois stratégies les plus souvent utilisées serait une bonne façon de maximiser le temps limité et la compréhension des données recueillies. La considération 
de toutes ces possibilités dans l'élaboration d'une étude contribuerait substantiellement à l'atteinte ultime d'une cueillette de donnée appropriée pour la clientèle pédiatrique.

\section{IMPLICATIONS CLINIQUES}

Le dépistage des stratégies d'adaptation mises en place par les enfants atteints d'une maladie chronique sert deux usages, le triage et la prévention. Dans un service clinique très sollicité, les ressources disponibles pour des interventions psychologiques peuvent être limitées. Par conséquent, les efforts d'intervention les plus intenses doivent être réservés aux patients chez qui la maladie entraîne une contrariété émotionnelle et comportementale importante. Les mesures brèves de dépistage, comme le Kidcope, permettent aux cliniques pédiatriques de faire des évaluations rapides, d'identifier très tôt les enfants qui éprouvent de la difficulté à s'adapter et d'établir des stratégies préventives.

En général, les évaluations de l'adaptation par le dépistage ou les listes peuvent être spécialement profitables pour les cliniciens puisqu'elles sont concises et faciles à administrer. Le Kidcope peut être employé pour évaluer l'adaptation dans différentes situations stressantes. Il procure également de l'information quant à la fréquence d'utilisation et à l'efficacité perçue d'une grande variété de stratégies cognitives et comportementales. Étant donné que des données normatives ont été recueillies pour le Kidcope, de l'information sur les stratégies d'adaptation spontanément adoptées par un ensemble diversifié d'enfants atteints de maladie chronique est donc disponible. De telles données seraient utiles dans l'évaluation des stratégies adaptatives puisque les données présentées dans ce chapitre suggèrent que les enfants atteints de maladie chronique et ceux atteints de maladie sévère se distinguent quant à la perception des stresseurs et aux stratégies qu'ils appliquent.

Plutôt que de juger si les enfants s'adapteront ou non aux stresseurs auxquels ils feront face, les cliniciens peuvent évaluer le type de stratégies dont disposent ces enfants en utilisant une mesure brève de dépistage. Les enfants atteints de maladie chronique qui utilisent un nombre restreint de stratégies peuvent dès le départ être ciblés afin de bénéficier d'une intervention, voire une intervention de groupe. Les recherches auprès d'enfants en santé et d'enfants atteints de maladie chronique suggèrent qu'une grande variété de stratégies d'adaptation disponible chez un individu est associée à un meilleur fonctionnement adaptatif (Knapp, Stark, Kurkjian et Spirito, 1991). Ce résultat appuie l'idée voulant que les enfants 
atteints de maladie chronique aient besoin de s'ajuster à différents types de demandes internes et externes tout au long de leur maladie et de leur traitement (Holroyd et Lazarus, 1982).

Les premiers efforts de prévention devraient d'abord s'organiser autour des enfants n'étant pas capables de générer de stratégie d'adaptation. Procurer de l'information sur la façon dont les autres enfants gèrent les différentes situations médicales liées à la maladie et au traitement constitue un exemple d'intervention éducative. Cette intervention peut être réalisée dans un contexte de résolution de problème qui aidera l'enfant à générer différentes stratégies pouvant servir de solutions possibles pour faire face à un problème lié à la maladie et au traitement. Par la suite, on peut suivre ces enfants afin de voir avec eux laquelle des stratégies d'adaptation leur semble la plus efficace pour diverses situations courantes durant la maladie (réajustement à l'école ou réaction à la taquinerie des pairs atteints de cancer). Malheureusement, ce qui est évident pour les cliniciens ne l'est pas nécessairement pour les patients ou les parents. Ainsi, lorsqu'un enfant gère de manière efficace un stresseur, il sera souvent nécessaire pour le clinicien de chercher avec lui les raisons de ce succès, en identifiant les stratégies utilisées (distraction). Le renforcement de cette réussite et du processus l'ayant provoquée peut encourager l'enfant et sa famille à utiliser des stratégies d'adaptation similaires lorsqu'une situation semblable se produira. Afin de faciliter cette pratique, il est possible de demander aux patients de reproduire le processus de résolution de problème lorsqu'ils seront confrontés à des situations potentiellement stressantes dans le futur.

Le moment idéal pour le dépistage initial de l'adaptation est presque tout de suite après le diagnostic de la maladie. À ce moment, l'enfant a habituellement été exposé à plusieurs stresseurs liés à la maladie et au traitement. Si l'enfant manifeste une difficulté croissante à faire face à ces stresseurs, il devrait être réévalué pour déterminer s'il a changé ses stratégies d'adaptation en réponse aux demandes différentes de la maladie ou du traitement. Les dépistages dans les moments de transition de la maladie (passer du traitement actif à la rémission dans le cas des patients cancéreux) et durant les transitions développementales (l'approche de l'adolescence pour les enfants souffrant de diabète) sont également recommandés.

L'expérience clinique propose que les enfants atteints de maladie chronique persistent à utiliser les mêmes types de stratégies d'adaptation lorsqu'ils sont confrontés à des procédures médicales stressantes et répétitives. Cependant, pour une proportion importante de ces enfants, les stratégies perdent de leur efficacité avec le temps. Un entraînement plus 
intensif à l'utilisation de certaines stratégies d'adaptation préexistantes ou de stratégies cognitives-comportementales spécifiques d'autocontrôle peut être prévu avec cette évaluation.

Finalement, hormis le moment où l'adaptation est évaluée selon la maladie, il est bon d'observer ou d'interviewer les enfants dans au moins deux situations différentes. D'abord, il est utile de faire une évaluation lorsque aucun stresseur majeur n'est anticipé prochainement afin de déterminer si l'enfant dispose de stratégies d'adaptation dans des circonstances optimales. Puis, il est bon de procéder à une autre évaluation juste avant que l'enfant soit confronté à un stresseur (une procédure médicale importante, une hospitalisation) pour déterminer s'il peut compter sur des stratégies d'adaptation dans des conditions stressantes.

\section{CONCLUSION}

L'évaluation standardisée de l'adaptation dans la pratique clinique était presque inexistante au moment où cette voie de recherche s'est ouverte. Le Kidcope a été volontairement élaboré de manière concise pour faciliter son usage par les cliniciens. Dans les douze années suivant sa publication, le Kidcope a été utilisé dans de multiples recherches. Cependant, il est difficile de dire si les professionnels de la santé mentale dépistent l'utilisation des stratégies d'adaptation parmi les enfants atteints de maladie chronique. Nous souhaitons que ce chapitre stimulera les psychologues en pédiatrie à réaliser des évaluations concises et standardisées de la capacité adaptative des enfants.

\section{TABLEAU 1}

\section{Catégories de stratégies d'adaptation}

1. Distraction

2. Retrait social

3. Restructuration cognitive

4. Autocritique

5. Blâmer les autres

6. Résolution de problème

7. Régulation émotionnelle

8. Pensée magique

9. Soutien social

10. Résignation 
KIDCOPE (version pour enfants plus âgés)

\begin{tabular}{|c|c|c|c|c|c|c|c|c|c|}
\hline \multirow[b]{2}{*}{$\begin{array}{l}\text { Instructions: Lis chaque énoncé et encercle } \\
\text { la phrase qui convient (s'il y en a). Ensuite, } \\
\text { réponds aux deux questions de droite pour } \\
\text { chacun des énoncés choisis, en encerclant } \\
\text { la meilleure réponse. }\end{array}$} & \multicolumn{4}{|c|}{$T^{\prime}$ arrive-t-il souvent de faire cela? } & \multicolumn{5}{|c|}{ Est-ce que cela t'a aidé? } \\
\hline & $\begin{array}{c}\text { Pas du } \\
\text { tout }\end{array}$ & Parfois & Souvent & $\begin{array}{l}\text { Presque } \\
\text { tout le } \\
\text { temps }\end{array}$ & $\begin{array}{c}\text { Pas du } \\
\text { tout }\end{array}$ & Un peu & Parfois & Assez & Très \\
\hline $\begin{array}{l}\text { 1. J'ai pensé à autre chose ; j'ai essayé } \\
\text { d'oublier; et/ou je suis allé faire autre } \\
\text { chose comme regarder la TV ou jouer } \\
\text { à un jeu pour ne plus y penser. }\end{array}$ & 0 & 1 & 2 & 3 & 0 & 1 & 2 & 3 & 4 \\
\hline $\begin{array}{l}\text { 2. Je suis demeuré loin des gens ; j'ai gardé } \\
\text { mes sentiments pour moi ; et j'ai géré } \\
\text { cette situation par moi-même. }\end{array}$ & 0 & 1 & 2 & 3 & 0 & 1 & 2 & 3 & 4 \\
\hline $\begin{array}{l}\text { 3. J'ai essayé de voir le bon côté des choses } \\
\text { et/ou je me suis concentré sur quelque } \\
\text { chose de positif qui pourrait ressortir } \\
\text { de cette situation. }\end{array}$ & 0 & 1 & 2 & 3 & 0 & 1 & 2 & 3 & 4 \\
\hline $\begin{array}{l}\text { 4. J'ai réalisé que je me suis mis tout le } \\
\text { problème sur le dos et que je me blâmais } \\
\text { pour l'avoir causé. }\end{array}$ & 0 & 1 & 2 & 3 & 0 & 1 & 2 & 3 & 4 \\
\hline
\end{tabular}




\begin{tabular}{|c|c|c|c|c|c|c|c|c|c|}
\hline \multirow[b]{2}{*}{$\begin{array}{l}\text { Instructions: Lis chaque énoncé et encercle } \\
\text { la phrase qui convient (s'il y en a). Ensuite, } \\
\text { réponds aux deux questions de droite pour } \\
\text { chacun des énoncés choisis, en encerclant } \\
\text { la meilleure réponse. }\end{array}$} & \multicolumn{4}{|c|}{$T^{\prime}$ arrive-t-il souvent de faire cela? } & \multicolumn{5}{|c|}{ Est-ce que cela t'a aidé? } \\
\hline & $\begin{array}{l}\text { Pas du } \\
\text { tout }\end{array}$ & Parfois & Souvent & $\begin{array}{l}\text { Presque } \\
\text { tout le } \\
\text { temps }\end{array}$ & $\begin{array}{l}\text { Pas du } \\
\text { tout }\end{array}$ & Un peu & Parfois & Assez & Très \\
\hline $\begin{array}{l}\text { 5. J'ai réalisé que quelqu'un d'autre avait } \\
\text { causé ce problème et je lui en veux } \\
\text { de m'avoir fait vivre cela. }\end{array}$ & 0 & 1 & 2 & 3 & 0 & 1 & 2 & 3 & 4 \\
\hline $\begin{array}{l}\text { 6. J'ai pensé à des façons de régler le } \\
\text { problème, en parlant aux autres pour } \\
\text { obtenir de l'information et connaître les } \\
\text { faits concernant le problème et/ou en } \\
\text { essayant de régler le problème lui-même. }\end{array}$ & 0 & 1 & 2 & 3 & 0 & 1 & 2 & 3 & 4 \\
\hline $\begin{array}{l}\text { 7a. J'ai parlé de ce que je ressentais, j’ai crié, } \\
\text { hurlé ou frappé quelqu'un. }\end{array}$ & 0 & 1 & 2 & 3 & 0 & 1 & 2 & 3 & 4 \\
\hline $\begin{array}{l}\text { 7b. J'ai tenté de me calmer en me parlant } \\
\text { à moi-même, en priant, en faisant des } \\
\text { marches ou juste en essayant de relaxer. }\end{array}$ & 0 & 1 & 2 & 3 & 0 & 1 & 2 & 3 & 4 \\
\hline $\begin{array}{l}\text { 8. Je n'arrêtais pas de penser et de souhaiter } \\
\text { que cela ne soit jamais arrivé; et/ou que } \\
\text { je pouvais changer ce qui venait d'arriver. }\end{array}$ & 0 & 1 & 2 & 3 & 0 & 1 & 2 & 3 & 4 \\
\hline
\end{tabular}


KIDCOPE (version pour enfants plus âgés) (suite)

\begin{tabular}{|c|c|c|c|c|c|c|c|c|c|}
\hline \multirow[b]{2}{*}{$\begin{array}{l}\text { Instructions: Lis chaque énoncé et encercle } \\
\text { la phrase qui convient (s'il y en a). Ensuite, } \\
\text { réponds aux deux questions de droite pour } \\
\text { chacun des énoncés choisis, en encerclant } \\
\text { la meilleure réponse. }\end{array}$} & \multicolumn{4}{|c|}{$T^{\prime}$ arrive-t-il souvent de faire cela? } & \multicolumn{5}{|c|}{ Est-ce que cela t'a aidé? } \\
\hline & $\begin{array}{l}\text { Pas du } \\
\text { tout }\end{array}$ & Parfois & Souvent & $\begin{array}{l}\text { Presque } \\
\text { tout le } \\
\text { temps }\end{array}$ & $\begin{array}{l}\text { Pas du } \\
\text { tout }\end{array}$ & Un peu & Parfois & Assez & Très \\
\hline $\begin{array}{l}\text { 9. Je me suis tourné vers ma famille, mes } \\
\text { amis ou d'autres adultes pour m'aider } \\
\text { à me sentir mieux. }\end{array}$ & 0 & 1 & 2 & 3 & 0 & 1 & 2 & 3 & 4 \\
\hline $\begin{array}{l}\text { 10. J'ai juste accepté le problème parce que } \\
\text { je savais que je ne pouvais rien y faire. }\end{array}$ & 0 & 1 & 2 & 3 & 0 & 1 & 2 & 3 & 4 \\
\hline
\end{tabular}




\section{NOTICES BIOGRAPHIQUES}

Élisabeth Chaussegros de Léry est étudiante au doctorat à la faculté d'éducation de l'Université de Sherbrooke. Ses intérêts de recherche portent sur les problématiques liées au stress chez les jeunes enfants, tels que l'attachement parent-enfant, l'adaptation biocomportementale, et les facteurs de risque. Son mémoire de maîtrise abordait la question du lien entre l'adaptation physiologique des enfants en contexte de nouveauté sociale et la relation d'attachement mère-enfant.

Sylvain Coutu est professeur au département de psychoéducation et de psychologie de l'Université du Québec à Hull. Ses intérêts de recherche touchent l'éducation préscolaire et la prévention des problèmes d'adaptation sociale des jeunes enfants. Ses travaux actuels portent sur la socialisation des émotions et le développement des compétences émotionnelles chez l'enfant d'âge préscolaire.

Deidre L. Donaldson est la directrice du Pediatric Psychology for May Institute, Inc. (Norwood, Massachusetts) et du Center for Children and Families at Saint Anne's Hospital (programme interdisciplinaire en clinique externe pour enfants ayant des besoins et recevant des soins de santé particuliers à Fall River, Massachusetts). Elle est aussi chercheure au Department of Psychiatry and Human Behavior at Brown University (Providence, Rhode Island). Ses premières recherches et intérêts cliniques portent sur les stratégies adaptatives des enfants soumis à des stresseurs médicaux, le suicide chez les jeunes et la diffusion des traitements validés dans divers milieux communautaires offrant des traitements.

Michelle Dumont est professeure au département de psychologie de 1'Université du Québec à Trois-Rivières. Ses intérêts de recherche portent sur les notions de stress, d'adaptation psychosociale et éducative, de résilience, de stratégie adaptative, de facteurs de risque et de protection au niveau individuel, familial et environnemental. Elle évalue ces différents thèmes de recherche selon une perspective ontogénétique. 
Jean-Luc Lambert est professeur à l'Université de Fribourg (Suisse), titulaire de la chaire de pédagogie curative, où il dirige une équipe de recherche et la formation des praticiens en praticiennes en éducation spécialisée. Il travaille depuis trente ans dans le domaine de la déficience intellectuelle. Ses thèmes de recherche actuels sont l'intervention précoce, l'éducation familiale et les troubles du comportement chez les adultes déficients.

Romain Lanners est assistant à l'Institut de pédagogie curative de l'Université de Fribourg (Suisse). Formé dans les domaines de l'intervention précoce et de l'éducation familiale, il termine sa thèse de doctorat sur la mise en place d'un financement des assurances sociales en fonction des besoins des familles d'enfants déficients.

Bernard Plancherel travaille comme chercheur dans l'unité de recherche au Service universitaire de psychiatrie de l'enfant et de l'adolescent à Lausanne (Suisse) et comme consultant et chargé de cours en méthodologie et statistiques au département de psychologie de l'Université de Fribourg (Suisse). Depuis quinze ans, il a travaillé dans la recherche sur l'adolescence et plus spécifiquement dans les domaines du stress, de la santé mentale, du coping et de l'estime de soi. Ses intérêts de recherche actuels portent sur les problèmes de dépendance à l'adolescence.

Marc A. Provost est professeur au département de psychologie de 1'Université du Québec à Trois-Rivières. Ayant réalisé des études postdoctorales à Keeles University sous la supervision de Corin Hutt, il s'est intéressé aux méthodes éthologiques de l'observation du comportement. Ses études portent sur le comportement social de l'enfant dans sa famille et en milieu de garde. Plus récemment, il s'intéresse au développement de problèmes du développement liés à l'internalisation et au retrait social.

Guadalupe Puentes-Neuman est psychologue et professeure adjointe au département d'éducation spécialisée (secteur adaptation scolaire) de l'Université de Sherbrooke. Elle s'intéresse à la formation des maîtres dans le domaine des interventions adaptées aux élèves en difficulté d'adaptation, dans une optique développementale. Ses intérêts de recherche portent sur le développement de la régulation socioaffective comme processus inhérent à l'adaptation de l'enfant lors des interactions sociales avec les parents et les pairs. Dans ce contexte, elle s'intéresse également à la relation parent-enfant (attachement, compétence parentale) comme lieu de développement de la régulation socioémotionnelle.

Nicole Royer est professeure au département des sciences de l'éducation de l'Université du Québec à Trois-Rivières. Elle s'intéresse au développement social et à l'adaptation sociale des enfants et des jeunes dans le 
contexte scolaire. Plus précisément, sa recherche porte en ce moment sur l'intégration des technologies et la pédagogie par projets auprès des enfants en difficulté d'apprentissage et des enfants en difficulté de comportement: elle s'intéresse aux effets de l'intervention sur la motivation scolaire, les habiletés sociales et la réussite éducative.

Anthony Spirito est professeur au Department of Psychiatry and Human Behavior of the Brown University School of Medicine (Providence, Rhode Island). Il a mené des recherches sur les stratégies adaptatives auprès de populations en pédiatrie. Ses recherches actuelles portent sur le traitement des adolescents manifestant des comportements à risque pour leur santé, comme la consommation d'alcool, l'usage de cigarettes ainsi que les comportements suicidaires.

Marcel Trudel est professeur en psychoéducation à la faculté d'éducation de l'Université de Sherbrooke. Il est également membre du Groupe de recherche sur les inadaptations sociales de l'enfance (GRISE). Il a participé à plusieurs projets de recherche longitudinale auprès d'enfants d'âge préscolaire. Ses intérêts de recherche s'orientent vers une perspective biosociale à l'étude du développement socioaffectif des jeunes enfants. Ses travaux ont également porté sur l'étude de l'organisation sociale des groupes de pairs tant sur le plan de la dominance sociale que sur le plan des rapports d'affiliation entre enfants. Il s'intéresse également aux effets des programmes d'intervention précoce sur le développement des enfants qui proviennent de milieux à risque.

Réjean Tessier est professeur titulaire à l'École de psychologie de l'Université Laval et membre du Centre de recherche du Pavillon Saint-François d'Assise, du Centre hospitalier universitaire de Québec. Il s'intéresse au développement des compétences sociales chez les enfants à risque sur les plans biologique et psychosocial et au rôle des premières relations familiales sur l'ajustement ultérieur de l'enfant. Ses travaux récents portent une attention particulière au rôle du père dans le développement de l'enfant et aux interactions parent-enfant dans différents contextes culturels. Il est co-directeur, avec George M. Tarabulsy, de la Collection d'Enfance.

George M. Tarabulsy est professeur adjoint au département d'études sur l'enseignement et l'apprentissage de la Faculté des sciences de l'éducation de 1'Université Laval. Il est aussi membre du Groupe de recherche en développement de l'enfant et de la famille (GREDEF) et du Groupe de recherche sur l'inadaptation psychosociale chez l'enfant (GRIP). Ses recherches portent sur le développement social, émotionnel et cognitif chez les familles à haut risque sur le plan psychosocial, ainsi que sur le 
développement de stratégies d'intervention adaptées à leurs besoins. Plus récemment, ses travaux ont porté sur le développement de la désorganisation de l'attachement et de l'inhibition comportementale chez les nourrissons et les enfants d'âge préscolaire. Il est codirecteur, avec Réjean Tessier, de la Collection d'Enfance. 


\section{BIBLIOGRAPHIE}

AARDAl-ERKSSON, E., KARlberg, B.E. et Holm, A.C. (1998). "Salivary cortisol: An alternative to serum cortisol determinations in dynamic function tests ". Clin-Chem-Lab-Med, 36, 215-222.

ACHENBACH, T.M. et EDELBROCK, C.S. (1981). “ Behavioral problems and competencies reported by parents of normal and disturbed children age four through sixteen ". Monographs of the Society for Research in Child Development, 46 (1, Serial No 188).

Ainge, D., Colvin, G. et BAKeR, S. (1998). "Analysis of perceptions of parents who have children with intellectual disabilities: Implications for service providers". Education and Training in Mental Retardation and Developmental Disabilities, 33, 331-341.

AlDwin, C.M. (1994). Stress, Coping, and Development. An Integrative Perspective. New York: The Guilford Press.

Altshuler, J. et Ruble, D.N. (1989). “Developmental changes in children's awareness of strategies for coping with uncontrollable stress ". Child Development, 60, 1337-1349.

Altshuler, J., Genevro, J.L., Ruble, D.N. et Bornstein, M.H. (1995). "Children's knowledge and use of coping strategies during hospitalization for elective surgery". Journal of Applied Developmental Psychology, 16, 53-76.

Auerbach, J., Lerner, Y., Barasch, M. et Palti, H. (1992). “ Maternal and environmental characteriwtics as predictors of child behavior problems and cognitive competence ». American Journal of Orthopsychiatry, 62, 409-420.

BAILEY, D.B. et SIMEONSSON, R.J. (1988). “ Assessing needs of families with handicapped infants ". Journal of Special Education, 22, 117-127.

BAiley, D.B., Aytch, I.S., OdOM, S.I., Symons, F. et Wolery, M. (1999). " Early intervention as we know it ". Mental Retardation and Developmental Disabilities, 5, 11-20. 
BAILEY, D.B., BLASCO, P.M. et SimeOnSSON, R.J. (1992). “ Needs expressed by mothers and fathers of young children with disabilities ". American Journal on Mental Retardation, 97, 1-10.

Baker, B.L., Blacher, J., Kopp, C.B. et Kraemer, S. (1997). “Parenting children with mental retardation". International Review of Research in Mental Retardation, 20, 1-45.

BAND, E.B. (1990). “Children's coping with diabetes: Understanding the role of cognitive development ». Journal of Pediatric Psychology, 15, $27-42$.

BARNARD, K.E. (1997). Influencing parent-child interactions for children at risk. Dans M.J. Guralnick (dir.), The Effectiveness of Early Intervention. Baltimore: Paul Brookes.

BAyley, D. et GarRalDA, M.E. (1987). "Children attending primary health care services: A study of recent life events ». Journal of the American Academy of Child and Adolescent Psychiatry, 26, 858864.

BAYLEY, D. et GARRALDA, M.E. (1990). “ Life-events: Children's reports ». Social Psychiatry et Psychiatry Epidemiology, 25, 283-288.

BECKMAN, P.J. (1983). "Influences of selected child characteristics on stress in families of handicapped infants ". American Journal on Mental Deficiency, 88, 150-156.

BECKMAN, P.J. (1991). “Comparison of mother's and father's perceptions of the effect of young children with and without disabilities ". American Journal on Mental Retardation, 95, 585-595.

Bedell, J.R., Giordani, B., Amour, J.L., TAvormina, J. et Boll, T. (1977). "Life stress and the psychological and medical adjustment of chronically ill children ". Journal of Psychosomatic Research, 21, 237-242.

Ben-Aryeh, H., Roll, R., Kahana, L., Malberger, E., Szargel, R. et GuTMAN, D. (1985). "Salivary as an indicator of stress ". International Journal of Psychosomatics, 32, 3-8.

BENES, F.M. (1994). “ Developmental changes in stress adaptation in relation to psychopathology". Development and Psychopathology, 6, 723-739.

Berg, C.A., MeEgan, S.P. et Deviney, F.P. (1998). “ A social-contextual model of coping with everyday problems across the lifespan ". International Journal of Behavioral Development, 22(2), 239-261. 
Berger, M., Bossert, S., Krieg, J.C., Dirlich, G., Ettmeier, W., Schreiber, W. et VON ZERSSEN, D. (1987). “Interindividual differences in the susceptibility of the cortisol system: An important factor for the degree of hypercortisolism in stress situations? " Biological Psychiatry, 22, 1327-1339.

BernzWeig, J., EISENBERG, N. et FABES, R.A. (1993). " Children's coping in self- and other-relevant contexts ". Journal of Experimental Child Psychology, 55, 208-226.

BiLlings, A.G. et Moos, R.H. (1981). " The role of coping responses and social resources in attenuating the stress of life events ". Journal of Behavioral Medicine, 4, 139-157.

BIRD, G.W. et HARRIS, R.L. (1990). “ A comparison of role strain and coping strategies by gender and family structure among early adolescents ". Journal of Early Adolescence, 10 (2), 141-158.

Bird, H.R., CANino, G., Rubio-Stipec, M., Gould, M.S. Ribera, J. Sesman, M., Woodbury, M., Huertas-Goldman, S., Pagan, A., SAnchezLACAY, A. et Moscoso, M. (1988). “ Estimates of the prevalence of chilhood maladjustment in a community survey in Puerto Rico ". Archives of General Psychiatry, 45, 1120-1126

BlanCHARD-Fields, F. et COLEMAN-IRION, J. (1988). “Coping strategies from the perspective of two developmental markers: Age and social reasoning ". Journal of Genetic Psychology, 149(2), 141-151.

BoekAerts, M. (1994, avril). "The other side of learning: Allocating resources to restore well-being » (ERIC Document No. ED 378 668). Paper presented at the annual conference of the American Educational Research Association. New Orleans, LA.

BoekAerts, M. (1996). "Coping with stress in childhood and adolescence ". Dans M. Zeidner and N.M. Endler (dir.), Handbook of Coping: Theory, Research, Applications, 20 (p. 452-484). Toronto: John Wiley et Sons, Inc.

Bouchard, G., SAbourin, S., Lussier, Y., Richer, C. et Wright, J. (1995). “ Nature des stratégies d'adaptation au sein des relations conjugales: présentation d'une version abrégée du Ways of Coping Questionnaire». Revue canadienne des sciences du comportement, 27(3), 371-377.

BOUCHARD, J.M. (1997). “ Famille et compétence éthique: de l'expert au partenaire chercheur ». Pages Romandes, 2, 5-18. 
Bouchard, J.M., Pelchat, D. et Boudreault, P. (1996). “ Les relations parents et intervenants: perspectives théoriques ". Apprentissage et Socialisation, 17, 21-34.

Boyce, W.T. et Jemerin, J.M. (1990). "Psychobiological differences in childhood stress response. I. Patterns of illness and susceptibility ». Developmental and Behavioral Pediatrics, 11, 196-206

Boyce, W.T., Alkon, A., Tschann, J.M., Chesney, M.A. et Alpert, B.S. (1995). “Dimensions of psychobiologic reactivity: Cardiovascular response to laboratory stressors in preschool children ". Annals of Behavioral Medecine, 17, 315-323.

Boyce, W.T., Jensen, E.W., CAssell, J.C., Collier, A.M., Smith, A.H. et RAIMEY, C.T. (1973). "Influence of life events and family routines on childhood respiratory tract illness ". Pediatrics, 60, 609-615.

Brazelton, T.B. (1984). Neonatal Behavioral Assessment Scale. London: Spastics International Medical Publications.

BRONFENBRENNER, U. (1977). “ Toward an experimental ecology of human development ». American Psychologist, 32, 513-531.

BRONFENBRENNER, U. (1979). The Ecology of Human Development: Experiments by Nature and Design. Cambridge: Harvard University Press.

BROTMAN BAND, E. et Weisz, J.R. (1988). “ How to feel better when it feels bad: Children's perspectives on coping with everyday stress". Developmental Psychology, 24(2), 247-253.

Brown, G.W., Sklair, F., HARris, T.O. et Birley, J.L.T. (1973). “ Life events and psychiatric disorders. Part I: Some methodological issues ". Psychological Medecine, 3, 74-87.

Brown, J.M., O'KeEFFe, J., SANDERS, S.H. et BAKer, B. (1986). “Developmental changes in children's cognition to stressful and painful situations ". Journal of Pediatric Psychology, 11, 343-357.

Brown, W.A. et Heninger, C. (1975). “Cortisol, growth hormone, free fatty acids, and experimentally evoked affective arousal ». American Journal of Psychology, 132, 1172-1176.

BRUCHON-SCHWEITZER, M. et DANTZER, R. (1994). Introduction à la psychologie de la santé. Paris: Presses universitaires de France.

Bull, B. et Drotar, D. (1991). "Coping with cancer in remission: Stressors and strategies reported by children and adolescents ". Journal of Pediatric Psychology, 16, 767-782 
Buysse, V., Wesley, P. et SkinNer, D. (1999). “ Community development approaches for early intervention ". Topics in Early Childhood Special Education, 19, 236-243.

CAIRNS, R.B. (1991). “ Multiple metaphors for a singular idea ». Developmental Psychology, 27, 23-26.

CAIRNS, R.B. (1998). “The making of developmental psychology ». Dans W. Damon (dir.), Handbook of Child Psychology: Theoretical Models of Human Development (Vol. 1). New York: Wiley.

CAIRnS, R.B. et CAIRNS, B.D. (1994). Lifelines and Risks: Pathways of Youth in our Time. New York: Cambridge University Press.

Cannon, W.B. (1929). Bodily Changes in Pain, Huger, Fear and Rage. New York: D. Appleton and Company.

CANNON, W.B. (1935). "Stresses and strains of homeostasis ". American Journal of Medical Sciences, (189), 1-14.

CAPALDI, K.A. et Patterson, G. R. (1990). “ Relation of parental transitions to boys' adjustment problems: I. A linear hypothesis. II. Mothers as risk for transmission and unskilled parenting ". Developmental Psychology, 27, 489-504.

CARR, J. (1974). " The effect of the severely subnormal on their families". Dans A.M. Clarke et A.D.B. Clarke (dir.), Mental Deficiency, the Changing Outlook (p. 807-839). London: Methuen.

Carroll, D. (1992). Health Psychology: Stress, Behavior and Disease. London: The Falmer Press.

Cartwright, S. et CoOper, C.L. (1996). " Coping in occupational settings ". Dans M. Zeidner et N.M. Endler (dir.), Handbook of Coping : Theory, Research, Applications, chap. 10 (p. 202-220). Toronto: John Wiley et Sons, Inc.

Carver, C.S., Scheier, M.F. et POzO, C. (1992). “Conceptualizing the process of coping with health problems ". Dans H.S. Friedman (dir.), Hostility, Coping, and Health (p. 167-199). Washington, DC: American Psychological Association.

CAUsey, D.L. et DubOW, E.F. (1992). “ Development of a self-report coping measure for elementary school children ". Journal of Clinical Child Psychology, 21, 47-59.

Chamove, A.S. et Bowman, R.E. (1978). “ Rhesus plasma cortisol response at four dominance positions ". Aggressive Behavior, 4, 43-55. 
Chrousos, G.P. et GolD, P.W. (1992). " The concepts of stress and stress system disorder: Overview of physical and behavioral homeostasis ". Journal of the American Medical Association, 267, 1244-1252.

Cicchetti, D. et Cohen, D. (1995). "Perspectives on developmental psychology ». Dans D. Cicchetti et D. Cohen (dir.), Developmental Psychopathology: vol 1. Theory and Method (p. 3-20). New-York: Wiley-Interscience.

Clayton, J.M., Glidden, L.M. et Kiphart, M.J. (1994). " The questionnaires on resources and stress: What do they measure? " American Journal on Mental Retardation, 99, 313-316.

CODDINGTON, R.D. (1972a). "The significance of life events as etiologic factors in the diseases of children. I - A survey of professional workers ". Journal of Psychosomatic Research, 16, 7-18.

CODDINGTON, R.D. (1972b). "The significance of life events as etiologic factors in the diseases of children. II - A study of a normal population". Journal of Psychosomatic Research, 16, 205-213.

COHEN, S. et EDWARDS, J. (1989). “ Personality characteristics as moderators of the relationship between stress and disorder ". Dans R.W.J. Neufeld (dir.), Advances in the Investigation of Psychological Stress (p. 234-283). New York: John Wiley and Sons.

CoHeN, S. et WiLls, T.A. (1985). "Stress, social support, and the buffering hypothesis". Psychological Bulletin, 98(2), 310-357.

COMPAS, B.E. (1987). " Coping with stress during childhood and adolescence ». Psychological Bulletin, 101(3), 393-403.

COMPAS, B.E. (1998). "An agenda for coping research and theory: Basic and applied developmental issues". International of Behavioral Development, 22(2), 231-237.

COMPAS, B.E. et WORSHAM, N. (1991, april). When Mom or Dad has cancer: Developmental Differences in Children's Coping with Family Stress. Paper presented at Society for research on child development meeting, Seattle, WA.

Compas, B.E., Banez, G.A., Malcarne, V. et Worsham, N. (1991). “Perceived control and coping with stress: A developmental perspective ». Journal of Social Issues, 47(4), 23-34.

COMPAS, B.E., MALCARNE, V.L. et FondaCARO, K.M. (1988). “ Coping with stressful events in older children and young adolescents". Journal of Consulting and Clinical Psychology, 56(3), 405-411. 
COWEN, E.L., WORK, W.C. et WYMAN, P.A. (1997). “ The Rochester child resilience project (RCRP): Facts found, lessons learned, future direction divined ". Dans S.S. Luthar, J.A. Burack, D. Cicchetti et J.R. Weisz (dir.), Developmental Psychopathology: Perspectives on Adjustment, Risk, and Disorder (p. 527-547). Cambridge: Cambridge University Press.

Coyne, J.C. et GotTlieb, B.P. (1996). “The mismeasure of coping by checklist ». Journal of Personality, 64, 959-991.

CURRY, S.L. et RUSS, S.W. (1985). “ Identifying coping strategies in children ". Journal of Clinical Child Psychology, 14(1), 61-69.

DAvis, E.P., DonZella, B., Krueger, W.K. et GunNAR, M.R. (1999). “ The start of a new school year: Individual differences in salivary cortisol responses in relation to child temperament ". Developmental Psychobiology, 35, 188-196.

Delongis, A., Coyne, J.C., DAKOF, G., Folkman, S. et LAZARUS, R.S. (1982). "Relationship of daily hassles, uplifts and major life events to health status ". Health Psychology, 1, 119-136.

Dettling, A.C., Gunnar, M.R. et Donzella, B. (1999). “ Cortisol levels of young children in full-day childcare centers: Relations with age and temperament ". Psychoneuroendocrinology, 24, 519-536.

DOHRENWEND, B.P. (1986). “ Note on a program of research on alternative social psychological models of relationships between life stress and psychopathology ". Dans M.H. Appley et R. Trumbull (dir.), Dynamics of Stress (p. 283-293). New York, Plenum Press.

Donaldson, D., Prinstein, M., Danovsky, M. et Spirito, A., (2000). "Patterns of children's coping with life stress ». American Journal of Orthopsychiatry, 70, 351-359.

Dumont, M. et Provost, M.A. (1999). “ The protective role of social support, coping strategies, self-esteem and social activities for adolescent's level of stress and depression". Journal of Youth and Adolescent: A Multi Disciplinary Research Publication, 28(3), 343363.

EbATA, A.T. et Moos, R.H. (1991). “ Coping and adjustment in distressed and healthy adolescents ". Journal of Applied Developmental Psychology, 12, 33-54. 
EbAtA, A.T. et Moos, R.H. (1994). “ Personal, situational, and contextual correlates of coping in adolescence ". Journal of Research on Adolescence, 4(1), 99-125.

EINFELD, S.L. et TONGE, B.J. (1996). “ Population prevalence of psychopathology in children and adolescents with intellectual disability: I. Rationale and methods". Journal of Intellectual Disability Research, 40, 91-98.

FARBER, B. (1960). “Family organization and crisis: Maintenance of integration in families with a severely mentally retarded child ". Monographs of the Society for Research in Child Development, 25, Serial No. 75.

FeLDMAN, M.A. et WALTON-ALLEN, N. (1997). “ Effects of maternal mental retardation and poverty on intellectual, academic, and behavioral status of school-age children ". American Journal on Mental Retardation, 101, 352-364.

FolKman, S. et LAZARUS, R.S. (1988). Ways of Coping Questionnaire: Research edition. Palo Alto, CA: Consulting Psychologists Press.

Forehand, R., ThOmas, A.M., Wierson, M., Brody, G. et Fauber, R. (1990). "Role of maternal functioning and parenting skills in adolescent functioning following parental divorce ". Journal of Abnormal Psychology, 99, 278-283.

Francis, S.F., WALKer, R., Riad-Fahmy, D., Hughes, D., Murphy, J.F. et GRAY, O.P. (1987). "Assessment of adrenocortical activity in term newborn infants using salivary cortisol determination ". Journal of Pediatrics, 111, 129-133.

FreUd, S. (1933). New Introductory Lectures on Psychoanalysis. New York: Norton.

GARBER, J. et Hilsman, R. (1992). " Cognitions, stress, and depression in children and adolescents". Dans D.P. Cantwell (dir.). Child and Adolescent Psychiatric Clinics of North America, Mood Disorders, 1(1), 129-167.

GarmezY, N. (1983). “Stressors of childhood». Dans N. Garmezy et M. Rutter (dir.), Stress, Coping and Development in Children (p. 43-84). Minneapolis: McGraw-Hill.

GARMEzy, N. et Nuechterlein, K. (1972). “ Invulnerable children: The fact and fiction of competence and disadvantage ». Child Development, 55, 97-111. 
Garmezy, N., MAsten, A.S. et Tellegen, A. (1984). “ The study of stress and competence in children: A building block for developmental psychopathology ". Child Development, 55, 97-111.

Ghaziuddin, M., Alessi, N. et Greden, J.F. (1995). “ Life events and depression in children with pervasive developmental disorders ". Journal of Autism and Developmental Disorders, 25, 495-502.

GIL, K.M., Williams, D.A., ThOMPSON, R.J. et KINNEY, T.R. (1991). "Sickle cell disease in children and adolescents: The relation of child and parent pain coping strategies to adjustment ". Journal of Pediatric Psychology, 16, 643-663.

GLIDDEN, L.M. (1993). “What we do not know about families with children who have developmental disabilities: Questionnaire on resources and stress as a case study ". American Journal on Mental Retardation, 97, 481-495.

Goldstein, S., Field, T. et HeAly, B.T. (1989). "Concordance of play behavior and physiology in preschool friends ". Journal of Applied Developmental Psychology, 10, 337-351.

Goodyer, I. (1994). “ Les événements existentiels dans l'enfance et l'adolescence ». Dans M. Bolognini, B. Plancherel, R. Nunez et W. Bettschart (dir.), La préadolescence : théorie, recherche et clinique (p. 89105). Paris : ESF.

GOODYER, I.M. (1996). “ Recent undesirable life events: Their influence on subsequent psychopathology". European Child et Adolescent Psychiatry, 5(Suppl 1), 33-37

Goodyer, I.M., KolviN, I. et GatZanis, S. (1985). “ Recent undesirable life events and psychiatric disorders of childhood and adolescence ». British Journal of Psychiatry, 47, 512-523.

Goodyer, I.M., Wright, C. et Altham, P.M.E. (1988). “ Maternal adversity and recent stressful life events in anxious and depressd children ". Journal of Child Psychology and Psychiatry, 29, 651-667.

Goodyer, I.M., Wright, C. et Altham, P.M.E. (1990). “ Friendships and recent life events in anxious and depressed school-age children". British Journal of Psychiatry, 156, 689-698.

GottlieB, G. (1973). Behavioral Embryology. New York: Academic Press.

Gottlieb, G. (1991). “Experiential canalization of behavioral development: Theory ». Developmental Psychology, 27, 4-13. 
Gottlieb, G. (1993). Individual Development and Evolution: The Genesis of Novel Behavior. New York: Oxford University Press.

GotTlieb, G., WAhLSTEN, D. et Lickliter, R. (1998). " The significance of biology for human development: A developmental psychobiological systems view ». Dans W. Damon (dir.), Handbook of Child Psychology: Theoretical Models of Human Development (Vol. 1). New York: Wiley.

Granger, D.A., Stansbury, K. et Henker, B. (1994). “Preschoolers' behavioral and neuroendocrine responses to social challenge". Merrill-Palmer Quarterly, 40, 190-211.

Granger, D.A., Weisz, J.R. et KAUNeCKIS, D. (1994). “ Neuroendocrine reactive, internalizing behavior problem and control-related cognitions in clinic-referred children and adolescents ". Journal of Abnormal Psychology, 103, 267-276.

Greaves, D. et PoOle, C. (1996). “ Mothers' observations of children with Down syndrome coping with demands to adapt ". Journal of Intellectual and Developmental Disability, 21, 153-162.

Greene, J.W., Walker, L.S., Hickson, G. et Thompson, J. (1985). "Stressful life events and somatic complaints inadolescents". Psychiatrics, 75, 19-22.

GUNNAR, M.R. (1989). "Studies of the human infant's adrenocortical response to potentially stressful events". Dans M. Lewis et J. Worobey (dir.), Infant Stress and Coping (p. 3-18). San Francisco: Jossey-Bass.

GUNNAR, M.R. (1992). “ Reactivity of hypothalamic-pituitary-adrenocortical system to stressors in normal infants and children ». Pediatrics, 90, 491-495.

GUNNAR, M.R. (1994). "Psychoendocrine studies of temperament and stress in early childhood: Expanding current models ». Dans J.E. Bates et T.D. Wachs (dir.), Temperament, Individual Differences at the Interface of Biology and Behavior (p. 175-198). Washington, DC: APA Science.

GunNar, M.R., ConNors, J., Isensee, J. et WAll, L. (1988). “ Adrenocortical activity and behavioral distress in human newborns ". Developmental Psychobiology, 21, 297-310. 
GunNAR, M.R., FISCH, R. et MALONE, S. (1984). “ The effects of pacifying stimulus of behavioural adrenocortical responses to circumcision". Journal of the American Academy of Child and Adolescent Psychiatry, 23, 34-38

GunNAR, M.R., FISCH, R., KORSVIK, S. et DONHOWE, J. (1981). “ The effects of circumcision on serum cortisol and behavior". Psychoneuroendocrinology, 6, 269-276.

GUNNAR, M.R., IsENSEE, J. et FUST, L.S. (1987). “ Adrenocortical activity and the Brazelton neonatal assessment scale: Moderating effects of the newborn's biomedical status ". Child Development, 58, 14481458.

Gunnar, M.R., MANGelsdorf, S., LARson, M. et HertsGaARd, L. (1989). "Attachment, temperament, and adrenocortical activity in infancy: A study of psychoendocrine regulation ". Developmental Psychology, 25, 355-363.

Gunnar, M.R., Marvinney, D., Isensee, J. et Fisch, R. (1989). “ Coping with uncertainty: New models of the relations between hormonal, behavioral, and cognitive processes ". Dans D.S. Palermo (dir.), Coping With Uncertainty: Behavioral and Developmental Perspectives (p. 101-129). Hillsdale, NJ: Erlbaum.

GUNNAR, M.R., RESNICK, J.S. et SNIDMAN, N. (1987). “ The physiology and psychology of behavioral inhibition in young children ". Child Development, 58, 1459-1473.

Gunnar, M.R., Tout, K., de HaAn, M., Pierce, S. et Stansbury, K. (1997). “ Temperament, social competence, and adrenocortical activity in preschoolers ". Developmental Psychobiology, 31, 65-85

Gust, D.A., Gordon, T.P., HAMbright, M.K. et Wilson, M.E. (1993). "Relationship between social factors and pituitary-adrenocortical activity in female rhesus monkeys (Macaca mulatta)". Hormones and Behavior, 27, 318-331.

HAAN, N. (1977). Coping and Defending. New York: Academic Press.

HARDY, D.F., POWER, T.G. et JAEDICKE, S. (1993). “ Examining the relation of parenting to children's coping with everyday stress ". Child Development, 64, 1829-1841.

HELFF, C.M. et GLIDDEN, L.M. (1998). “ More positive or less negative? Trends in research on adjustment of families rearing children with developmental disabilities ". Mental Retardation, 36, 457-464. 
HenRY, J.P. et StePHENS, P.M. (1977). Stress, Health and the Social Environment: A Sociobiologic Approach to Medecine. New York, Springer Verlag.

Hill, R. (1949). Families Under Stress. New York: Harper et Brothers.

Hodges, K., Kline, J.J., Barbero, G. et Flanery, R. (1984). “ Life events occurring in families of children with recurrent abdominal pain ". Journal of Psychosomatic research, 28, 185-188.

HOFFNER, C. (1993). “Children's strategies for coping with stress: Blunting and monitoring ". Motivation and Emotion, 17(2), 91-106.

Holahan, C.J., Moos, R.H. et Schaefer, J.A. (1996). “Coping, stress resistance, and growth: Conceptualizing adaptative functioning". Dans M. Zeidner et N.M. Endler (dir.), Handbook of Coping: Theory, Research, Applications, chap. 2 (p. 24-43). Toronto: John Wiley et Sons, Inc.

HOLMES, T.H. et RAHE, R.H. (1967). " The social readjustment of stress ». Journal of Psychosomatic Research, 11, 213-218.

HOLMES, T.H. et RAHE, R.H. (1967). "The social readjustment rating scale ». Journal of Psychosomatic Research, 4, 189-194.

HOLROYD, K.A. et LAZARUS, R.S. (1982). “Stress, coping and somatic adaptation ". Dans C. Goldberger et S. Breznitz (dir.), Handbook of Stress: Theoretical and Clinical Aspects (p. 21-35). New York: Free Press.

Hubert, W. et de JONG-Meyer, R. (1989). “ Emotional stress and saliva cortisol response ". Journal of Clinical Chemy and Clinical Biochem., 27, p. 235-237.

JACOBS, T.J. et CHARLES, E. (1980). “Life events and the occurrence of cancer in children ". Psychosomatic Medecine, 42, 11-24.

JEMERIN, J.M. et BOYCE, W.T. (1992). “Cardiovascular markers of biobehavioral reactivity". Journal of Developmental and Behavioral Pediatrics, 13, 46-49.

Jensen, P.S., Richters, J., Ussetry, T., Bloedau, L. et DAVID, H. (1991). "Child psychopathology and environmental influences. Discrete life events versus ongoing adversity". Journal of the American Academy of Child and Adolescent Psychiatry, 30, 303-309.

Jeunier, B., Trudel, M. et Legendre, A. (1997). “Étude biosociale des modes d'adaptation en groupe de pairs". Enfance, (2), 247-267. 
JoHnson, J. (1986). Life events as Stressors in Childhood and Adolescence. Newbury Park, CA: Sage.

JOHNSON, J.H. et MCCUCHEON, S.M. (1980). “ Assessing life stress in older children and adolescents: Preliminary findings with life events checklist ». Dans I.G. Sarason et C.D. Spielberger (dir.), Stress and Anxiety, Washington, DC: Hemisphere.

KAGAN, J., REZNICK, J.S. et SNIDMAN, N. (1987). "The physiology and psychology of behavioral inhibition in young children». Child Development, 58, 1459-1473.

KAGAN, J., REZNICK, J.S. et SNIDMAN, N. (1988). "Biological bases of childhood shyness ». Science, 240, 167-171.

KAHN, J.P., RubinOw, D.R., DAvis, C.L., KLING, M. et Post, R.M. (1988). "Salivary cortisol: A practical method for evaluation of adrenal function ». Biological Psychiatry, 23, 335-349.

Kanner, A.D., Coyne, J.C., SChaefer, C. et Lazarus, R.S. (1981). "Comparison of two modes of stress measurement: Daily hassles and uplifts versus major life events ". Journal of Behavioral Medecine, 4, 1-39.

KANNer, A.D., Feldman, S.S., Weinberger, D.A. et Ford, M. (1987). "Uplifts, hassles, and adaptational outcomes in early adolescents". Journal of Early Adolescence, 7, 41, 371-394.

KASLOW F. (1990). “Divorce therapy and mediation for better custody". Japanese Journal of Family Psychology, 4, 19-37.

KeOGH, B.K., Garnier, H.E., Bernheimer, L.P. et Gallimore, R. (2000). "Models of child-family interactions for children with developmental delays: Child-driven or transactional? " American Journal on Mental Retardation, 105, 32-46.

KIRK, R.E. (1982). Experimental Design: Procedures for the Behavioral Sciences (2e édition). Belmont, California: Brooks/Cole publishing services.

KLIEWER, W. et SANDLER, I.N. (1993). “Social competence and coping among children of divorce ". American Journal of Orthopsychiatry, 63(3), 432-440.

KNAPP, L., Stark, L.J., KURKJiAN, J. et SPIRITO, A. (1991). “Assessing coping in children and adolescents: Research and practice ". Educational Psychology Review, 3, 309-334. 
KOHN, P.M. (1996). “ On coping adaptively with daily hassles». Dans M. Zeidner et N.M. Endler (dir.), Handbook of Coping: Theory, Research, Applications, chap. 9 (p. 181-201). Toronto: John Wiley et Sons, Inc.

KopP, C.B. (1989). "Regulation of distress and negative emotions: A developmental view ». Developmental Psychology, 25, 343-354.

Kovacs, M. (1980). "Rating scales to assess depression in school-aged children ". Acta Paedopsychiatrica, 46, 305-315.

KraUSS, M.W. (1997). "Two generations of family research in early intervention ". Dans M.J. Guralnick (dir.), The Effectiveness of Early Intervention (p. 611-624). Baltimore: Paul Brookes.

Kruesi, M.J.P., SCHMidT, M.E., DONNELly, M., HibBS, E.D. et HAMBURGER, S.D. (1989). "Urinary free cortisol output and disruptive behavior in children ". Journal of the American Academy of Child and Adolescent Psychiatry, 28, 441-443.

Laborit, H. (1979). L'inhibition de l'action. Paris: Masson.

LaFreniere, P.J., Dubeau, D., Janosz, M. et Capuano, F. (1990). “ Profils socio-affectifs des enfants d'âge préscolaire ». Revue canadienne de psycho-éducation, 19, 23-41.

LaFreniere, P.J., Provost, M.A. et Dubeau, D. (1992). “ From an insecure base: Parent-child relations and internalizing behavior in the preschool ". Early Development and Parenting, 1(3),137-148.

LAMBERT, J.L. (1978). “Problèmes liés à l'annonce du handicap chez des parents d'enfants mongoliens ". Bulletin de psychologie scolaire et d'orientation, 1, 13-19.

LAMBERT, J.L. (1980). “La formation des parents d'enfants arriérés mentaux à la modification du comportement ". Journal de thérapie comportementale de langue française, 2, 27-35.

LAMBERT, J.L. (1995). “ Retard mental et adaptation familiale. Réflexions pour la recherche et l'intervention ". Dans H. Lejeune (dir.), Des animaux et des hommes. Hommage à Marc Richelle (p. 129-156). Paris: Presses universitaires de France.

LAMBERT, J.L. et LAMBERT-BOITE, F. (1993). Éducation familiale et handicap mental. Fribourg: Éditions Universitaires.

LAMBERT, J.L. et STUDER, F. (1998). “ Les pères des jeunes enfants déficients intellectuels ». Sauvegarde de l'enfance, 3, 131-137. 
Lambert, J.L., Piret, M., Scohy, C. et Lalière, C. (1993). “ Sources de stress et de ressources dans des familles de jeunes enfants déficients mentaux ". Sauvegarde de l'enfance, 3, 285-299.

LAndon, J., Smith, D.S., Perry, L.A. et Al-Ansari, A.A.K. (1982). “ The assay of salivary cortisol ". Dans G.F. Read, D. Riad-Fahmy, R.F. Walker et K. Griffiths (dir.), Immunoassays of Steroids in Saliva (p. 300-307). New York: Alpha Omega Publishing.

LANNERS, R. (1997). Étude du processus d'adaptation. De la notion de stress aux notions de besoins, de stratégies et de réactions émotionnelles. Université de Fribourg: mémoire de licence (non publié).

LANNERS, R. et LAMBERT, J.L. (1999). “Die Bedürfnisse des Eltern behinderter Kleinkinder ". Vierteljahresschrift für Heilpädagogik und ihre Nachbargebiete, 68, 36-47.

LAZArus, R.S. (1966). Psychological Stress and the Coping Process. New York: McGraw-Hill.

LAZARUS, R.S. et COHEN, J.B. (1980). “ Environmental stress ». Dans I. Altman et J.F. Wohlwill (dir.), Human Behavior and the Environment: Current Theory and Research. New York: Plenum.

LAZARUs, R.S. et FolKMAN, S. (1984). Stress, Appraisal, and Coping. New York: Springer.

LAZARUS, R.S. et LAUMIER, R. (1978). “ Stress-related transactions between person and environment ". Dans L.A. Pervin et M. Lewis (dir.), Internal and External Determinants of Behavior. New York: Plenum.

LAZARUS, R.S., KANNER, A.D. et FOlKMAN, S. (1980). “ Emotions: A cognitive-phenomenological analysis ». Dans R. Plutchik et H. Kellerman (dir.), Theories of Emotions. Vol. 1. Emotions: Theory, Research, and Experience. New York: Academic Press.

Legendre, A. et Trudel, M. (1996). “ Cortisol and behavioural responses of young children in a group of unfamiliar peers ". Merrill-Palmer Quarterly, 42, 554-577.

LENGUA, L.J. et SANDLER, I.N. (1996). “ Self-Regulation as a moderator of the relation between coping and symptomatology in children of divorce ». Journal of Abnormal Child Psychology, 24(6), 681-701. 
LePORE, S.J. et EvANS, G.W. (1996). "Coping with multiple stressors in the environnement ". Dans M. Zeidner et N.M. Endler (dir.), Handbook of Coping: Theory, Research, Applications, chap. 16 (p. 350377). Toronto: John Wiley et Sons, Inc.

LERNER, R.M. (1984). On the Nature of Human Plasticity. New York: Cambridge University Press.

LERNER, R.M. (1991). “Changing organism-context relations as the basic process of development: A developmental contextual perspective ". Developmental Psychology, 27, 27-32.

LEVINE, S. (1983). " A psychobiological approach to the ontogeny of coping ". Dans N. Garmezy et M. Rutter (dir.), Stress, Coping and Development in Children (p. 107-131). New York: McGraw-Hill.

LEVINE, S. (1993). "The influence of social factors on the response to stress ". Psychotherapy and Psychosomatics, 60, 33-38.

LEVINE, S. et COE, C.L. (1985). " The use and abuse of cortisol as measure of stres ". Dans T. Field, P. McCabe et N. Schneiderman (dir.), Stress and Coping (p. 149-159). Hillsdale, NJ: Erlbaum.

Levine, S., CoE, C.L. et Wiente, S.G. (1989). “ Psychoneuroendocrinology of stress: A psychobiological perspective». Dans F.R. Bush et S. Levine (dir.), Psychoneuro-endocrinology. New York: Academic Press.

Levine, S., Wiener, S.G., Coe, C.L., Bayart, F.E.S. et Hayashi, K.T. (1987). " Primate vocalization: A psychobiological approach ». Child Development, 58, 1408-1419.

LeWIS, M., THOMAS, D.A. et WOROBEY, J. (1990). “ Developmental organization, stress, and illness ". Psychological Science, 1, 316-318.

LILLIE, T. (1993). "A harder thing than triumph: Roles of fathers of children with disabilities ». Mental Retardation, 31, 438-443.

LÔO, P. et LÔO, H. (1995). Le stress permanent. Paris: Masson, Médecine et psychothérapie.

Losoya, S., EISENBERG, N. et FABES, R.A. (1998). “Developmental Issues in the Study of Coping ". International Journal of Behavioral Development, 22(2), 287-313. 
Lundberg, U., DeChateau, P., WindberG, J. et Frankenhaeuser, $M$. (1981). "Catecholamine and cortisol excretion patterns in threeyear-old children and their parents ". Journal of Human Stress, 7, 311.

Magnusson, D. (1988). Individual Development from an Interactional Perspective: A longitudinal Study. Hilldale, NJ : Erlbaum.

Magnusson, D. (1998). "The logic and the implication of a personoriented approach». Dans R.B. Cairns, L.R. Bergman et J. Kagan (dir.), Methods and Models for Studying the Individual. Thousand Oaks: Sage.

Mahoney, G. et Bella, J.M. (1998). "An examination of the effects of family-centered early intervention on child and family outcomes ". Topics in Early Childhood Special Education, 18, 83-95.

MANogue, K.R., Leshner, A.I. et CANDlAND, D.K. (1975). “ Dominance status and adrenocortical reactivity to stress in squirrel monkeys (Saimiri sciureus) '. Primates, 16, 457-463.

Mantagos, S., Moustogiannis, A. et VAgenAKis, A.G. (1998). “ Diurnal variation of plasma cortisol levels in infancy ". Journal of Pediatry, Endocrinology and Metabolism, 11, 549-553

MASH, E.J. et TERDAL, L.G. (1988). “ Behavioral assessment of childhood disturbance ". Dans E.J. Mash et L.G. Terdal (dir.), Behavioral Assessment of Childhood Disorders (2e édition, p. 3-78). New York: Guilford Press.

MASON, J.W. (1959). “ Psychological influences on the pituitary-adrenal cortical system ". Dans G. Pincus (dir.), Recent Progress in Hormone Research. New York: Academic Press

MASON, J.W. (1968). “A review of psychoendocrine research on pituaryadrenal cortical system ". Psychosomatic Medicine, 30, 576-608

MASTEN, A.S. (1989). "Resilience in development: Implications of the study of successful adaptation for developmental psychopathology ". Dans D. Cicchetti (dir.), Rochester Symposium on Developmental Psychopathology, vol. 1 (p. 261-294). Hillsdale: LEA.

MASTEN, A.S. et GARMEZY, N. (1985). “Risk, vulnerability and protective factors in developmental psychopathology ». Dans B.B. Lahey et A.E. Kazdin (dir.), Advances in Clinical Child Psychology, 8 (p. 1-52). New York: Plenum Press. 
Masten, A.S., Hubbard, J.J., Gest, S.D., Tellegen, A., Garmezy, N. et RAMireZ, M. (1999). "Competence in the context of adversity: Pathways to resilience and maladaptation from childhood to late adolescence ». Developmental and Psychopathology, 11, 143-169.

MCBRide, S.L. et Peterson, C. (1997). “ Home-based early intervention with families of children with disabilities: Who is doing what? " Topics in Early Childhood Special Education, 17, 209-224.

MCBURnetT, K., LAHEY, B.B., FRICK, P.J., RISCH, C., LOEBER, R., HART, E.L., CHRIST, M.A.G. et HANSON, K.S. (1991). “ Anxiety, inhibition, and conduct disorder in children: II. Relation to salivary cortisol ". Journal of the American Academy of Child and Adolescent Psychiatry, 30, 192-196.

McBurnett, K., LAhey, B.B., Rathouz, P.J. et Loeber, R. (2000). “ Low salivary cortisol and persistent aggression in boys referred for disruptive behavior ". Archives of General Psychiatry, 57, 38-43.

MCCubbin, H.I. et PAtTerson, J. (1983). “ Family transition: Adaptation to stress ". Dans H.I. McCubbin et C.R. Figley (dir.), Stress and the Family. Coping with Normative Transitions (p. 5-23). New York: Brunner et Mazel.

MCGREW, W.C. (1972). An Ethological Study of Children's Behavior. New York: Academic Press.

MCLEAN, P. (1980). “Depression as a specific response to stress ". Dans I.G. Sarason et C.D. Spielberger (dir.), Stress and Anxiety, Washington: Hemisphere.

Meaney, M.J., AitKen, D.H., Bhatnager, S., VAN Berkel, C. et SAPOlsky, R.M. (1988). "Effect of neonatal handling on age-related impairments associated with the hippocampus ». Science, 239, 766-768

Meaney, M.J., Aitken, D.H., ViaU, V., Sharma, S. et Sarrieau, A. (1989). “Neonatal handling alters adrenocortical negative feedback sensitivity and hippocampal type II glucocorticoid receptor binding in the rat ». Neuroendocrinology, 50, 597-604

MENEGHAN, E. (1982). “ Measuring coping effectiveness: A panel analysis of marital problem and coping efforts ". Journal of Health and Social Behavior, 23, 220-234. 
Miller, S., Sherman, H., Combs, C. et KruUs, L. (1992). " Patterns of children's coping with short term medical and dental stressors: Nature, complications, and future directions ". Dans A.M. LaGreca, L.J. Siegel, J. Wallander et C. Walker (dir.), Stress and Coping in Child Health (p. 157-190). New York: Guilford.

MinNES, P.M. (1988). “ Family stress associated with a developmentally handicapped child". Dans N. Ellis et N. Bray (dir.), International Review of Research in Mental Retardation (vol. 15, p. 195-226). New York: Academic Press.

MischarA, B. (1987). Traduction française de Ways of Coping Checklist. Manuscrit inédit. Montréal: Université du Québec à Montréal.

Montagner, H., Henry, J.C., Lombardot, M., Benedini, M., Burnod, J. et NiCOLAS, R.M. (1978). "Behavioral profiles and corticosteroid excretion rhythms in young children. Part 2: Circadian and weekly rhythms in corticosteroid excretion levels of children as indicators of adaptation to social context ". Dans V. Reynolds et N.G. Blurton Jones (dir.), Human Behavior and Adaptation (p. 229-265). London: Francis et Taylor.

Montagner, H., Restoin, A. et Henry, J.C. (1982). “ Biological defense rhythms, stress, and communication in children". Dans W.W. Hartup (dir.), Review of Child Development Research (p. 291-319). Chicago, Il: University of Chicago Press.

MUIR RyAN, N. (1989). “Stress-coping strategies identified from school age children's perspective ". Research in Nursing and Health, 111122 .

NiHIRA, K., WeISNER, T.S. et BERNHEIMER, L.P. (1994). “ Ecocultural assessment in families of children with developmental delays: Construct and concurrent validities ". American Journal on Mental Retardation, 98, 551-566.

O'LeARY, A. (1990). “Stress, emotion, and human immune function". Psychological Bulletin, 108, 363-382.

ORR, R.R., CAMERON, S.J. et DAY, D.M. (1991). “Coping with stress in families with children who have mental retardation: An evaluation of the double ABCX model ». American Journal on Mental Retardation, 95, 444-450. 
PARkeR, J.M. et EndLeR, N.S. (1996). “ Coping and defense: A historical overview ". Dans M. Zeidner and N.M. Endler (dir.), Handbook of Coping: Theory, Research, Applications, chap. 1 (p. 3-23). Toronto: John Wiley et Sons, Inc.

PAtTerson, J. et MCCubBIN, J. (1983). “ ACOPE-adolescent coping orientation for problem experiences ". Manuscrit non publié. Madison: University of Wisconsin.

PATterson, J.M. (1993). "The role of family meanings in adaptation to chronic illness and disability ». Dans A.P. Turbull, J.M. Patterson, S.K. Behr, D.L. Murphy, J.G. Marquis et M.J. Blue-Banning (dir.), Cognitive Coping, Families, and Disability (p. 221-238). Baltimore: Paul Brookes.

Paulhan, I. et Bourgeois, M. (1995). Stress et coping: les stratégies d'ajustement à l'adversité. Paris: Presses universitaires de France.

Paykel, E.S., Myers, J.K., Dienelt, M.N., Klerman, G.L., Lindenthal, J.J. et PePPeR, M.P. (1969). "Life events and depression ». Archives of General Psychiatry, 21, 753-760.

PeArlin, L. et SCHOOleR, G. (1978). "The structure of coping ". Journal of Health and Social Behavior, 19, 2-21.

PeARLIN, L.I. (1983). “ Role strains and personal stress ». Dans H.B. Kaplan (dir.), Psychosocial Stress (p. 3-32). New York: Academic Press.

Perrez, M. et Reicherts, M. (1992). Stress, Coping, and Health: A Situation-behaviour Approach. Theory, Methods, Applications. Seattle: Hogrefe et Huber Publishers.

Perrez, M., Plancherel, B. et AbabKov, V. (2000). “ Nouvelle approche pour l'étude du stress dans les interactions familiales». Dans J.P. Pourtois et H. Desmet (dir.), Relation familiale et résilience (p. 217253). Paris: L'Harmattan.

PfefFer, C.R. (dir.) (1996). Severe Stress and Mental Disturbance in Children. Washington, DC: American Psychiatric Press.

Plancherel, B., Bettschart, W., Bolognini, M., Dumont, M. et HalFON, O. (1997). “ Influence comparée des événements existentiels et des tracas quotidiens sur la santé psychique à la préadolescence». Neuropsychiatrie de l'enfance et de l'adolescence, 45 (3), 126-138. 
Plancherel, B., NúÑez, R., Bolognini, M., Leidi, C. et BetTschart, W. (1992). “L'évaluation des événements existentiels comme prédicteurs de la santé psychique à la préadolescence ". Revue européenne de psychologie appliquée, 42, 3, 229-239.

Plancherel, B., Bolognini, M. et NúÑez, R. (1994). “ L'hypothèse de l'effet buffer à la préadolescence ». Dans M. Bolognini, B. Plancherel, R. Núñez et W. Bettschart (dir.), Préadolescence: théorie, recherche et clinique (p. 159-172). Paris: ESF Editeur.

Price, D.A., Close, G.C. et Fielding, B.A. (1983). “ Age of appearance of circadian rhythm in salivary cortisol values in infancy ». Archives of Disease in Childhood, 58, 454-456.

Prinstein, M.J., LA GrecA, A.M., VernberG, E.M. et Silverman, W.K. (1996). “Children's coping assistance: How parents, teachers, and friends help children cope after a natural disaster ". Journal of Clinical Child Psychology, 25(4), 463-475.

Provost, M.A. (1995). “ Le soutien social, de l'enthousiasme à la réalité ». Dans M. A. Provost (dir.), Le soutien social: quelques facettes d'une notion à explorer (p. 5-22). Montréal: Behaviora.

Provost, M.A. et LAFreniere, P.J. (1991). “ La relation entre la qualité de l'attachement parents-enfants et la compétence sociale à la garderie ». Dans. M.A. Provost et R.E. Tremblay (dir.), Famille, inadaptation sociale et intervention (p.66-86). Montréal: Les éditions Agence d'Arc.

RAHE, R.H. (1968). “ Life change measurement as a predictor of illness ". Proceedings of the Royal Society of Medicine, 61, 1124-1126.

RiAD-FAHMY, D., ReAD, G.F. et Hughes, I.A. (1983). “Corticosteroids ”. Dans C.H. Gray et H.T. James (dir.), Hormones in Blood (vol. 4, p. 285-315). New York: Academic Press.

RiAD-FAHMY, D., READ, G.F., WALKer, R.F. et GRIFFITHS, K. (1982). “ Steriods in saliva for assessing endocrine function". Endocrine Reviews, 3, 367-395.

Richters, J. et Weintraub, S. (1990). “Beyond diathesis: Toward an understanding of high-risk environments ". Dans J. Rolf, A.S. Masten, D. Cicchetti, K. Nuechterlein et S. Weintraub (dir.), Risk and Protective Factors in the Development of Psychopathology (p. 6796). Cambridge: Cambridge University Press. 
Rivolier, J. (1989). L'Homme stressé. Paris: Presses universitaires de France, coll. “Psychologie d'aujourd'hui ".

RosenZWeIG, M.R. et LEIMAN, A.L. (1989). Psychophysiologie (traduit par D. Bélanger) (2 édition). Ville Mont-Royal: Décarie.

Roth, S. et COHEN, L.J. (1986). " Approach, avoidance, and coping with stress ». American Psychologist, 41(7), 813-819.

RUtTeR, M. (1987). "Psychosocial resilience and protective mechanisms ». American Journal of Orthopsychiatry, 57, 316-331.

Rutter, M. et StandberG, S. (1992). “ Psychosocial stressors: Concepts, causes and effects ". European Child et Adolescent Psychiatry, 1, 313.

SANDler, I.N., WolchiK, S.A., MACKinnon, D., AYers, T.S. et RoOsA, M.W. (1997). " Developing linkages between theory and intervention in stress and coping processes ". Dans S.A. Wolchik et I.N. Sandler (dir.), Handbook of Children's Coping: Linking Theory and Intervention (p. 3-39). New York: Plenum Press.

SAPOLSKY, R.M. (1982). " The endocrine stress-response and social status in the wild baboon ". Hormones and Behavior, 16, 279-292.

SAPOLSKY, R.M. (1990). “ Adrenocortical function, social rank, and personality among wild baboons ". Biological Psychiatry, 28, 862-878.

SAPOLSKY, R.M. et RAY, J. (1989). "Styles of dominance and their endocrine correlates among wild olive baboons (Papio anubis) ». American Journal of Primatology, 18, 1-13.

SCALleT, A.C., SuOMI, S.J. et Bowman, R.E (1981). "Sex differences in adrenocortical response to controlled agonistic encounters in rhesus monkeys ". Physiology and Behavior, 26, 385-390.

SCERbO, A.S. et KolKO, D.J. (1994). " Salivary testosterone and cortisol in disruptive children: Relationship to aggressive, hyperactive, and internalizing behaviors". Journal of the American Academy of Child and Adolescent Psychiatry, 33, 1174-1184.

SCHMIDT, N.A. (1998). "Salivary cortisol testing in children ". Issues in Comprehensive Pediatric Nursing, 20, 183-190.

SChOWAlter, J.E. (1983). "Psyche and soma of physical illness during adolescence ». Psychosomatics, 24, 453-461. 
Schulz, K.P., Halperin, J.M., NewCORn, J.H., Sharma, V. et Gabriel, S. (1997). " Plasma cortisol and aggression in boys with ADHD ». Journal of the American Academy of Child and Adolescent Psychiatry, 36, 605-609.

SCHWARZER, R. et SCHWARZER, C. (1996). “ A critical Survey of Coping Instruments ". Dans M. Zeidner et N.M. Endler (dir.), Handbook of Coping: Theory, Research, Applications, chap. 6 (p. 107-132). Toronto: John Wiley et Sons, Inc.

SeIfFGE-KRenKe, I. (1994a). “ Le Coping à l'adolescence: âge, sexe et différence culturelle ». Dans M. Bolognini, B. Plancherel, R. Nuñez et W. Bettschart (dir.), Préadolescence: théorie, recherche et clinique (p. 173-183). Paris: ESF Éditeur.

SEIFFGE-KRENKE, I. (1994b). “ Les modes d'ajustement aux situations stressantes du développement: Comparaison d'adolescents normaux et d'adolescents perturbés ". L'orientation scolaire et professionnelle, 23(3), 313-327.

SeIfFGe-Krenke, I. et Shulman, S. (1990). “ Coping style in adolescence: A cross-cultural study ». Journal of Cross-Cultural Psychology, 21, 351-377.

SeIFFGE-Krenke, I. et Shulman, S. (1993). “Stress, coping, and relationships in adolescence ». Dans S. Jackson et H.R. Tomé (dir.), Adolescence and its Social Worlds (p. 169-196). Hove: Lawrence Erlbaum Associates.

Selye, H. (1936). “A syndrome produced by diverse nocuous agents ". Nature, 138, 32.

Selye, H. (1953). The Stress of Life. New York: McGraw-Hill.

Selye, H. (1956). Le stress de la vie. Paris: Gallimard.

Simeonsson, R.J. et BAILEY, D.B. (1993). " Family dimensions in early intervention ". Dans S.J. Meisels et J.P. Shonkoff (dir.), Handbook of Early Childhood Intervention (p. 428-444). Cambridge: Cambridge University Press.

SKINNER, E. et EDGE, K. (1998). “ Reflections on Coping and Development across the Lifespan ". International Journal of Behavioral Development, 22(2), 357-366.

SMITH, E. (1952). "Emotional factors as revealed in the intake process with parents of defective children". American Journal of Mental Deficiency, 56, 806-812. 
Smith, K., Ackerson, J., Blotcky, A. et Berkow, R. (1990). “ Preferred coping styles of pediatric cancer patients during invasive medical procedures ». Journal of Psychosocial Oncology, 8, 59-70.

SPANGLER, G. et SCHEUBECK, R. (1993). “ Behavioral organization in newborns and its relation to adrenocortical and cardiac activity ". Child Development, 64, 622-633.

SpIeLberger, C.D. (1979). Understanding Stress and Anxiety (p. 47). New York: Harper and Row.

SPIRITO, A. (1996). "Commentary: Pitfalls in the use of brief screening measures of coping ». Journal of Pediatric Psychology, 4, 573-575.

Spirito, A., Francis, G., Overholser, J. et Frank, N. (1996). “ Coping, depression, and adolescent suicide attemps ". Journal of Clinical Child Psychology, 25, 147-155.

Spirito, A., Stark, L.J. et Overholser, J. (1989). “ Common problems and coping strategies II. Findings with adolescent suicide attempts". Journal of Abnormal Child Psychology, 17, 213-221.

SPIRITO, A., STARK, L.J. et TYC, V. (1996). " Coping strategies described during hospitalization by chronically ill children ». Journal of Child Psychology, 23, 314-322.

SPIRITO, A., STARK, L.J. et Williams, C. (1988). “ Development of a brief checklist to assess coping in pediatric patients ". Journal of Pediatric Psychology, 13, 555-574.

SPIRITO, A., STARK, L., GiL, K. et TYC, V. (1995). “ Coping with everyday and disease-related stressors by chronically ill children and adolescents ". Journal of the American Academy of Child and Adolescent Psychiatry, 34, 283-290.

Spirito, A., Stark, L.J., Grace, N. et Stamoulis, D. (1991). " Common problems and coping strategies reported in childhood and early adolescence ». Journal of Youth and Adolescence, 20(5), 531-544.

StANSBURY, K. et GUNNAR, M.R. (1994). "Adrenocortical activity and emotion regulation". Monographs of the Society for Research in Child Development, 59 (2-3), 250-283.

Stark, L.J., Spirito, A., Williams, C. et GuÈvremont, D. (1989). "Common problems coping strategies I: Findings with normal adolescents ». Journal of Abnormal Child Psychology, 17, 203-212. 
STEIN, R.E. et JESSOP, D.J. (1982). “ A noncategorical approach to chronic childhood illness ". Public Health Reports, 97, 361-378.

Steinhausen, H.C. et RADTKe, B. (1986). “ Life events and child psychiatric disorders ". Journal of American Academy for Child Psychiatry, 25, 125-129.

Stephenson, W. (1953). The Study of Behavior: Q Technique and its Methodology. Chicago, Il: University of Chicago Press.

StONE, A.A. et NeAle, J.M. (1984). “ New measure of daily coping: Development and preliminary results ". Journal of Personality and Social Psychology, 46, 892-906.

StorA, J.B. (1997). Le stress. Paris: Presses universitaires de France, coll. “Que sais-je? ».

Stores, R., Stores, G., Dellows, B. et Buckley, S. (1998). “Daytime behaviour problems and maternal stress in children with Down's syndrome, their siblings, and non-intellectually disabled and other intellectually disabled peers ". Journal of Intellectual Disability Research, 42, 228-237.

STRACK, S. et FEIFEL, H. (1996). “Age differences, coping, and the adult life span ". Dans M. Zeidner et N.M. Endler (dir.), Handbook of Coping: Theory, Research, Applications, chap. 21 (p. 485-501). Toronto: John Wiley et Sons, Inc.

STRAYER, F.F. (1980). “ Child ethology and the study of preschool social relations ". Dans H.C. Foot, A.J. Chapman et J.R. Smith (dir.), Friendship and Social Relations in Children (p. 235-266). New York: Wiley.

Suls, J. et Fletcher, B. (1985). "The relative efficacy of avoidant and nonavoidant coping strategies: A meta-analysis ". Health Psychology, 4, 249-258.

SWEARINGEN, E.M. et COHEN, L.H. (1985). “ Measurement of adolescents' life events: The junior high life experiences survey ". American Journal of Community Psychology, 13, 69-85.

TAYLOR, S.E. (1995). Health Psychology. Montréal: McGraw-Hill, Inc.

TenNes, K. et KREYe, A. (1985). “Childen's adrenocortical responses to classroom activities and tests in elementary school". Psychosomatic Medicine, 47, 451-460. 
TENNES, K. et MASON, J. (1982). “ Developmental psychoendocrinology: An approach to the study of emotions ". Dans C. Izard (dir.), Measuring Emotions in Infants and Children (p. 21-37). New York: Cambridge University Press.

Tennes, K., Kreye, M., Avitable, N. et Wells, R. (1986). “ Behavioral correlates of excreted catecholamines and cortisol in second grade children ". Journal of the American Academy of Child and Adolescent Psychiatry, 25, 764-770.

TERRY, D.J. (1994). “Determinants of coping: The role of stable and situational factors". Journal of Personality and Social Psychology, $66(5), 895-910$.

THOITS, P.A. (1983). « Dimensions of life events that influence psychological distress: An evaluation and synthesis of the literature ». Dans H.B. Kaplan (dir.), Psychosocial Stress: Trends in Theory and Research. New York: Academic Press.

THOMPson, R.A. (1991). "Emotional regulation and emotional development . Educational Psychology Review, 3, 269-307.

Tobin, D.L., Holroyd, K.A. et ReynOlds, R.V.C. (1984). “ Manual for Coping Strategies Inventory ". Manuscrit non publié, Ohio University.

TRUDEL, M. (1998, juillet). “ Adrenocortical aspects of behavioural adaptation within novel peer groups ». Texte présenté au symposium "Sources of social adjustment: Ecological constraints, physiological regulation and behavioural accommodation" de la International Society for the Study of Behavioural Development (ISSBD), Berne, Switzerland.

Trudel, M., Gauthier, R., Jacques, M. et Strayer, F.F. (1983). “ L'organisation sociale chez les jeunes enfants en garderie: I. Les rapports et les rôles affiliatifs au sein du groupe ". Apprentissage et Socialisation, 6, 233-247.

TyszKowa, M. (1990). " Coping with difficult school situations and stress resistance». Dans H. Bosma et S. Jackson (dir.), Coping and Self Concept in Adolescence (p. 187-202). Berlin: Springer Verlag.

URSIN, H. (1980). "Personality, activation and somatic health. A new psychosomatic theory». Dans S. Levine et H. Ursin (dir.), Coping and Health (p. 259-280). New York: Plenum Press.

URsin, H., Baade, E. et Levine, S. (1978). Psychobiology of Stress : A Study of Coping Mens. New York: Academic Press. 
VAILLANT, G.E. (1971). “Theoretical hierarchy of adaptive Ego-mechanisms ». Archive of General Psychiatry, 24: 101-118

VAillant, G.E. (1977). Adaptation to Life. Boston: Little, Brown.

Vanyukov, M.M., Moss, H.B., Plail, J.A., Blackson, T., MezziCH, A.C. et TARTER, R.E. (1993). "Antisocial symptoms in preadolescent boys and in their parents: Associations with cortisol". Psychiatry Research, 46, 9-17.

Villemain, F. (1989). Stress et immunologie. Paris: Presses universitaires de France, coll. “ Nodules ».

VIRKUNNEN, M. (1985). “Urinary free cortisol secretion in habitually violent offenders ". Acta Psychiatric Scandinavia, 72, 40-44.

Vitaro, F., Gagnon, C. et Tremblay, R.E. (1992). “ Liens d'amitié et fonctionnement social chez les enfants rejetés ». Enfance, 1-2, 113-127.

Walker, C.D., Bodnar, M., Forget, M.-A., Toufexix, D.J. et Trottier, G. (1997). "Stress et plasticité neuroendocrinienne". Sciences Médecine, 13, 509-518.

WALlenstein, J.S. (1985). " Children of divorce: Preliminary report of a ten-year follow-up of older children and adolescents ". Journal of the American Academy of Child and Adolescent Psychiatry, 24, 545553.

WATERS, E. et SROUfe, A.L. (1983). “ Social competence as developmental construct ». Developmental Review, 3, 79-97.

WERNER, E.E. et SMITH, R.S. (1992). Overcoming the Odds: High Risk Children from Birth to Adulthood. New-York: Cornell University Press.

WeSTLING, D.L. (1996). “What do parents of children with moderate and severe mental disabilities want?" Education and Training in Mental Retardation and Developmental Disabilities, 4, 86-114.

WIDOM, C.S. (1999). "Posttraumatic stress disorder in abused and neglected children grown up ". American Journal of Psychiatry, 156(8), 1223-1229.

Wierzbicki, M. et CARver, D. (1989). “ Children's engagement in antidepressive activities ". Journal of Genetic Psychology, 150, 163-174.

WilloughBy, J.C. et GLIDDEN, L.M. (1995). “ Fathers helping out: shared child care and marital satisfaction of parents of children with disabilities ". American Journal on Mental Retardation, 99, 399-406. 
WILLS, T.A. (1986). “Stress and coping in early adolescence: Relationships to substance use in urban school samples ". Health Psychology, 5, 503-529.

WiLls, T.A. et HiRKY, A.E. (1996). "Coping and substance abuse: A theorical model and review of the evidence ». Dans M. Zeidner et N.M. Endler (dir.), Handbook of Coping: Theory, Research, Applications, chap. 13 (p. 279-302). Toronto: John Wiley et Sons, Inc.

WoOdMan, D.D., HinTon, J.W. et O'Neill, M.T. (1978). “ Cortisol secretion and stress in maximum security hospital patients ". Journal of Psychosomatic Research, 22, 133-136.

WoOlston, J.L., Gianfredi, S., Gertner, J.M., PAugus, J.A. et Mason, J.W. (1983). "Salivary cortisol: A nontraumatic sampling technique for assaying cortisol dynamics ". Journal of the American Academy of Child and Adolescent Psychiatry, 22, 474-476.

Wyman, P.A., CoWen, E.L., Work, W.C., Hoyt-Meyers, L., MaGnUs, K.B. et FAGEN, D.B. (1999). "Caregiving and developmental factors differentiating young at-risk urban children showing resilient versus stress-affected outcomes: A replication and extension". Child Development, 70, 645-659.

ZEIDNER, M. et SAKLOFSKE, D. (1996). "Adaptive and Maladaptive Coping ". Dans M. Zeidner and N.M. Endler (dir.), Handbook of Coping: Theory, Research, Applications, chap. 22 (p. 505-531). Toronto: John Wiley et Sons, Inc. 


\section{INDEX ONOMASTIQUE}

$\boldsymbol{A}$

Aardal-Erksson, E., 35

Achenbach, T.M., 78

Ainge, D., 110

Aldwin, C.M., 4

Altshuler, J., 65, 73, 79, 81

Auerbach, J., 24

B

Bailey, D.B., 107, 111

Baker, B.L., 99, 110, 139

Band, E.B., 148

Barnard, K.E., 120

Bayley, D., 24

Beckman, P.J., 109

Bedell, J.R., 24

Ben-Aryeh, H., 15

Benes, F.M., 37

Berg, C.A., 56, 64

Berger, M., 16

Bernzweig, J., 66

Billings, A.G., 135

Bird, G.W., 21

Bird, H.R., 25

Blanchard-Fields, F., 73

Boekaerts, M., 67

Bouchard, G., 79

Bouchard, J.M., 107, 111, 122

Boyce, W.T., 24, 34, 40

Brazelton, T.B., 39

Bronfenbrenner, U., 7, 103

Brotman Band, E., 67, 79, 81, 82, 88

Brown, G.W., 24

Brown, J.M., 139, 141

Brown, W.A., 40

Bruchon-Schweitzer, M., 33
Bull, B., 144

Buysse, V., 111

C

Cairns, R.B., 31, 51

Cannon, W.B., 3, 11, 32

Capaldi, K.A., 86

Carr, J., 95

Carroll, D., 32

Cartwright, S., 65

Carver, C.S., 62

Causey, D.L., 124, 148

Chamove, A.S., 37

Chrousos, G.P., 40

Cicchetti, D., 71, 86

Clayton, J.M., 98

Coddington, R.D., 19

Cohen, S., 21, 72

Compas, B.E., 18, 24, 27, 61, 65, 67, $73,74,144$

Cowen, E.L., 85

Coyne, J.C., 147

Curry, S.L., 65, 135, 141

\section{D}

Davis, E.P., 43

DeLongis, A., 21

Dettling, A.C., 45

Dohrenwend, B.P., 16

Donaldson, D., 145

Dumont, M., 72, 74

$\boldsymbol{E}$

Ebata, A.T., 60, 61, 65, 138

Einfeld, S.L., 109 
$\boldsymbol{F}$

Farber, B., 94

Feldman, M.A., 124

Folkman, S., 61, 79, 80

Forehand, R., 86

Francis, S.F., 36

Freud, S., 57

\section{G}

Garber, J., 25

Garmezy, N., 71, 72, 75, 76

Ghaziuddin, M., 124

Gil, K.M., 143

Glidden, L.M., 96

Goldstein, S., 45

Goodyer, I.M., 25, 26

Gottlieb, G., 31, 51

Granger, D.A., 36, 43, 48

Greaves, D., 124, 125

Greene, J.W., 24

Gunnar, M.R., 35, 38, 39, 42, 43, 44, 46

Gust, D.A., 37

\section{$\boldsymbol{H}$}

Haan, N., 57

Hardy, D.F., 124, 125

Helff, C.M., 112

Henry, J.P., 15

Hill, R., 99

Hodges, K., 24

Hoffner, C., 65

Holahan, C.J., 57, 60, 66

Holmes, T.H., 19, 76, 77

Holroyd, K.A., 150

Hubert, W., 15

\section{$\boldsymbol{J}$}

Jacobs, T.J., 24

Jemerin, J.M., 34

Jensen, P.S., 24

Jeunier, B., 41

Johnson, J., 19, 21, 23

Johnson, J.H., 20
K

Kagan, J., 40

Kahn, J.P., 36

Kanner, A.D., 21, 22

Kaslow, F., 24

Keogh, B.K., 107

Kirk, R.E., 82

Kliewer, W., 63

Knapp, L., 149

Kohn, P.M., 61

Kopp, C.B., 40

Kovacs, M., 25

Krauss, M.W., 123

Kruesi, M.J.P., 47

\section{$\boldsymbol{L}$}

Laborit, H., 16

LaFreniere, P.J., 75, 77

Lambert, J.L., 94, 95, 96, 97, 101, $104,105,106,109,113,115,120$, 122,124

Landon, J., 35

Lanners, R., 108, 118

Lazarus, R.S., 3, 6, 13, 21, 33, 56, 60, $72,73,81,87,94,97,117,132$, $133,134,144$

Legendre, A., 41, 49

Lengua, L.J., 63

Lepore, S.J., 62

Lerner, R.M., 31, 51

Levine, S., 35, 38, 46, 51

Lewis, M., 34

Lillie, T., 109

Lôo, P., 22

Losoya, S., 64

Lundberg, U., 43

\section{M}

Magnusson, D., 50, 51

Mahoney, G., 122

Manogue, K.R., 37

Mantagos, S., 35

Mash, E.J., 133

Mason, J.W., 38

Masten, A.S., 7, 74, 75, 87 
McBride, S.L., 113

McBurnett, K., 47

McCubbin, H.I., 100

McGrew, W.C., 49

McLean, P., 21

Meaney, M.J., 37

Meneghan, E., 62

Miller, S., 148

Minnes, P.M., 99

Montagner, H., 43, 44, 45

Muir Ryan, N., 66

\section{N}

Nihira, K., 104

\section{O}

O'Leary, A., 35

Orr, R.R., 100

\section{$\boldsymbol{P}$}

Parker, J.M., 60

Patterson, J., 133

Patterson, J.M., 118

Paulhan, I., 4, 57, 58

Paykel, E.S., 24

Pearlin, L., 56, 62

Pearlin, L.I., 21

Perrez, M., 27, 117, 118

Pfeffer, C.R., 23

Plancherel, B., 17, 20, 21, 72

Price, D.A., 35

Prinstein, M.J., 66

Provost, M.A., 75, 86

$\boldsymbol{R}$

Rahe, R.H., 16

Riad-Fahmy, D., 36

Richters, J., 85

Rivolier, J., 15, 17, 23

Rosenzweig, M.R., 34

Roth, S., 60, 61

Rutter, M., 22, 24, 26, 71, 77

\section{$\boldsymbol{S}$}

Sandler, I.N., 59

Sapolsky, R.M., 37
Scallet, A.C., 37

Scerbo, A.S., 47

Schmidt, N.A., 36

Schulz, K.P., 47

Schwarzer, R., 59, 60, 61

Seiffge-Krenke, I., 65, 73

Selye, H., 3, 12, 16, 32

Simeonsson, R.J., 107

Skinner, E., 62, 68

Smith, E., 93

Smith, K., 140

Spangler, G., 39

Spielberger, C.D., 13

Spirito, A., 67, 73, 132-138, 142, 146

Stansbury, K., 46

Stark, L.J., 134, 135, 136, 139

Stein, R.E., 142

Steinhausen, H.C., 24

Stephenson, W., 41

Stone, A.A., 135

Stora, J.B., 18

Stores, R., 124

Strack, S., 68

Strayer, F.F., 41

Suls, J., 141

Swearingen, E.M., 20

\section{T}

Taylor, S.E., 6

Tennes, K., 38, 44, 47

Terry, D.J., 61

Thoits, P.A., 26

Thompson, R.A., 40

Tobin, D.L., 133

Trudel, M., 41

Tyszkowa, M., 19

$\boldsymbol{U}$

Ursin, H., 16, 34

V

Vaillant, G.E., 12, 57, 58

Vanyukov, M.M., 47

Villemain, F., 16

Virkunnen, M., 46

Vitaro, F., 77 
$\boldsymbol{W}$

Walker, C.D., 35

Wallenstein, J.S., 24

Waters, E., 72, 73, 74, 75

Werner, E.E., 72

Westling, D.L., 108

Widom, C.S., 23

Wierzbicki, M., 138
Willoughby, J.C., 101, 109

Wills, T.A., 60, 136

Woodman, D.D., 46

Woolston, J.L., 36

Wyman, P.A., 71, 72

$\boldsymbol{Z}$

Zeidner, M., 57, 61, 62, 63 


\section{INDEX THÉMATIQUE}

$\boldsymbol{A}$

ABCX, 7, 99, 100

ACBX, 7, 99, 101, 102

ACOPE, 133, 134

ACTH, 15, 34

activation endocrinienne, 39, 48

activité

adrénocorticale, 38, 44

cognitive, 67

comportementale, 44

distrayante, 67

endocrinienne, 38,42

évitante, 67

neuroendocrinienne, 45

physiologique, 45

physique, 67

adaptation, 3-8, 12-14, 26, 29, 31, 32,

35-37, 39-41, 44, 46, 48-51, 55, 56,

58-60, 62-64, 69, 71, 72, 75, 77,

$78,81,89,93,96,97,99,101$, $105-107,110-112,117,121,123-$

$127,131-138,140-151$

comportementale/endocrinienne/

physiologique, 6, 31, 36, 37, 40, 41,51

familiale, 93, 96, 97, 99, 101, 105$107,110,117,121,123,124,127$

positive/négative, 146, 147

psychosociale, 7, 32, 50, 56, 59, $63,77,89$

adolescence, 19, 48, 57, 63, 64, 73, $106,135,150$

Adolescent-Coping Orientation for

Problem Experiences Inventory, 133 adolescent, 8, 13, 19-21, 23, 26, 27, $67,72,73,104,129,132,134,135$, $137-143,146$

dépressif, 137, 138 suicidaire/non-suicidaire, 136, 137 adrénaline, 14, 15, 33, 34

adrénocorticale, 38

adversité, 7, 31, 32, 38, 46, 51, 66, 68,

$71,76,86,88$

familiale, 84,86

psychosociale, 7

agressivité, 16, 46, 47, 94, 99, 126

aire hypophysaire/ hypothalamique,

33,34

ajustement

comportemental et physiologique, 33

familial, 95

analyse

de validité, 113

de variance, 17,82

factorielle, 108, 118, 146, 147

angoisse, 12, 16, 21, 23, 57, 94

anticipation, 57, 68

anxiété, 14, 23, 25, 26, 43, 58, 61, 62, $66,68,119,138,141,144$

appréciation

cognitive, 98

de l'événement stressant, 97, 116

primaire/secondaire, 97

apprentissage, 35, 73, 108, 115

approche

centrée sur les groupes, 74, 75

centrée sur les variables, 74

contextuelle, 6, 56-58

de type psychopathologique, 95

observationnelle, 41

psychodynamique, 6, 56-58

sociométrique, 45

asthme, 22, 24, 138

attente, 72, 86, 98, 109, 111

attribution de signification, 7, 112 
autocontrôle, 151

autocritique, 137-141, 143-145

auto-efficacité, 27

auto-évaluation positive, 97

autorégulation, 74

axe hypothalamique-pituitaire-surrénal, 6,34

\section{$\boldsymbol{B}$}

besoin, 7, 66, 86, 94, 96, 97, 101, 105, $107,109,110,112,118,121-123$, 141,150

bien-être, 4, 8, 17, 20, 33, 34, 56, 57, $62,68,94$

biologie, 4, 36, 51

blâmer les autres, 135, 137, 143-145

"buffer" ou effet tampon, 16, 17, 21

burn out, 18

\section{C}

cancer, 24, 138, 144, 150

capacité de résolution de problème, $86,87,97$

caractéristique

biocomportementale, 49

individuelle, 16, 43, 55, 59, 62

catécholamine, 6, 26, 33

cauchemar, 21, 23

centre

d'intervention précoce, 118 spécialisé, 99, 100

changement développemental, 56

check-list, 67

chercheur, 5, 13, 31, 34, 40, 41, 43, $46,48,49,67,71,85,93,95-99$, $102,107,110,112,117,118,124$, $125,131-133,142,146-148$

cible d'intervention, 8,55

circonstance stressante, 68

clinicien, 131, 140, 142, 145, 147, 149-151

cognition, 5, 12, 58, 62, 80, 90

cohésion familiale, 97

colère, 26, 138, 141

comorbidité, 25, 26, 47

compétence, 42, 64, 68, 71, 72, 74,

$75,79,84,88,123,127$ intellectuelle, 64, 68

socio-affective, 74, 76, 124, 125

comportement, 4, 12, 16, 22, 23, 25,

31-33, 36-39, 41, 43-51, 53, 58-60,

$62,72,74,76,90,98,100,108$,

$124,126,140,141$

dépendant, 23, 25

d'inhibition, 41

dysfonctionnel, 46, 89

régressif, 23

social, 41, 43, 44

concept de soi, 27, 63

concept de risque, 88

condition de vie stressante, 71

conduite éducative, 109, 115, 116

conflit, 19, 57, 71, 94, 146

contexte

de résolution de problème, 150

écologique, 32

familial, 27, 49

social, $31,42,50,66,68$

contrainte matérielle et financière, 102

contrôle interne ou externe, 59

coping, 3-8, 12, 13, 16, 27, 32, 38, 39, 55-68, 73, 74, 79-81, 90, 93-95, 97, $98,102,110,112,116,117,119$,

$123-127,131,146$

actif/passif, 61, 64

approche/évitement, 60

centré sur le problème, $58,60,61$, $63,66,67,74$

centré sur les émotions, 58, 60,

$61,63,66,67$

cognitif, 64, 65, 67

d'affrontement, 60

d'assistance émotionnelle, 66

évitant, 61, 63

familial, 116

maternel, 73

social, 65-67

Coping Strategy Inventory, 133

cortex cérébral, 37

corticolibérine, 34, 37

corticostérone, 34

corticosurrénale, 34

cortisol, 15, 26, 34-39, 41-51, 53

plasmatique, 37

salivaire, $15,35,36,47$ 
urinaire, 47

crainte, 44

crise, 59, 61, 68, 95, 96, 99-101

croissance, 35,37

croyance

à propos de soi et du monde, 56

laïque ou religieuse, 122

parentale, 97

CSI, 133, 134

\section{D}

danger, 4, 12, 14, 57

décharge émotionnelle, 125

défense immunitaire, 15, 16

psychologique, 16

défi, 14, 57, 58, 62, 118, 120

déficience intellectuelle, 3, 7, 91-93, $97,98,101,102,107,108,110$,

$117,118,120,123,124$

déficience immunitaire, 32

déficit d'attention, 25, 47

degré, 39, 42, 47, 65, 84, 138, 140, 142

d'efficacité, 59

de contrôlabilité, 61,68

de satisfaction conjugale, 97, 104

demande, 6, 7, 12, 13, 56, 57, 68, 80, 97-99, 102, 103, 107-113, 118, 120$123,127,132,150$

d'aide aux professionnels, 121

de moyen financier, 108

de soutien, 108

des familles, 107, 111

en information, 108, 109

endogène, 57

externe ou interne, 56, 98, 150

liée à l'intégration sociale, 108

pour la recherche de services adaptés, 108

démarche éducative, 106

déni, 57, 60, 61, 94

dépistage, $75,131,142,147-150$

dépression, 14, 15, 22-26, 63, 119,

$124,137,138$

déséquilibre, 4, 96, 101, 138

désorganisation familiale, 112 détresse, 5, 6, 17, 38, 61-64, 66, 73, $94,136,138-142$

développement, 3, 7, 18, 22, 24, 27, $31,36,37,40,43,50,51,55,58$, 60, 62-64, 68, 71-74, 84, 85, 89, 99-101, 103, 106-108, 111, 113, $118,122-124,131,132,134-136$ cognitif, 22, 55

épigénétique, 51 familial, 94

diabète, 15, 22, 24, 138, 150

différence

individuelle/contextuelle, 4, 11, 40, 42, 98

interindividuelle, 64

situationnelle, 144

difficulté cognitive, affective

et sociale, 127

difficulté comportementale, 109

discorde familiale, 22, 24

distanciation, 79, 93

distraction, 60, 61, 65, 67, 132, 133, $137,139,144,150$

cognitive, 65

comportementale, 65

distress, 12

divorce, 18, 22, 24, 57

dominance sociale, 37, 45

douleur, 15, 24, 112, 132, 139, 140, 144

dramatisation, 141

dysfonctionnement physiologique, 46

\section{$\boldsymbol{E}$}

échantillon psychiatrique, 131

échelle, 20, 77, 79, 81, 104, 107, 110, $113,115,118,119,125,132,147$, 148

d'internalisation et/ou

d'externalisation, 77,78

école, 18, 19, 43, 67-69, 76, 79, 89, 99, 103-106, 108, 109, 111, 136, 145,150

écologie, 97, 104, 116

familiale, 97, 110, 115, 116

matérielle, 105

écosystème, 103, 106, 111, 116 
éducation, 93, 99, 103, 104, 109, 111, $118,120,122,124,127$

familiale, 93, 103, 120, 122, 127

effet de médiation, 17

effet du stress, 17, 19, 27, 73

efficacité, 6, 8, 55, 58, 60-62, 96, 101, $105,121,132,134,138,139,149$, 150

des ressources, 96, 101

du coping, 6, 8, 55, 60-62, 134

effort, 8, 44, 56, 60, 89, 131, 149, 150

cognitif et comportemental, 56 , $60,73,98$

pour faire face, 59,66

émotion, 5, 11, 12, 23, 38, 40, 53, 58, $60,63-65,74,77,80,90,93,101$, $119,122,123,138$

empathie, 63, 72

endosystème, 104, 116

énergie, 15, 34, 35, 111

enfance, 5, 6, 11, 19, 40, 55-57, 63-66, 73

enfant, 3-8, 13, 17-21, 23-27, 29, 31, $32,35,36,38-51,53,55,63-69$, 71-90, 93-94, 96, 98-101, 103-113, $115,116,118-127,129,131,132$, $135,138-152$

à haut risque, 83

aberrant, 79, 83, 87, 88

agressif et turbulent, 48

amical, 42

anxieux, 141

autiste, 100, 124

compétent, $82,86-88$

déficient intellectuel, 8, 93, 95100, 102, 106-109, 112, 113,

$115,117,120,122,-127$

hospitalisé, 145

hyperactif avec déficit d'attention, 47

inhibé, 40

non déficient, 124, 125

résilient, 68, 74, 82, 83, 86-88

vulnérable, 86

entretien individuel, 80

entrevue, 67, 79, 81, 88, 138, 148

semi-structurée, 81,147

structurée, 66, 79 environnement, 51

éducatif, 115

physique, 107, 115

psychosocial de la famille, 72

social et physique, 56

épuisement, 16

équilibre interne, 33, 35

équilibre physiologique, 33

équilibre physique et psychologique, 61,62

estime de soi, 5, 7, 59, 62, 63, 72

état

émotionnel, 38, 62

endogène, 50

homéostasique, 33

physiologique, 32

étiologie, 107, 113

eustress, 12

évaluation, 4, 13, 14, 19, 36, 39, 45, $50,56,57,89,96,97,106,110$,

$115-117,134,140,148,149,151$

clinique, 110

cognitive, 56, 57, 97, 98

de programme, 89

du stress, 96

sociométrique, 45

standardisée, 151

événement, 4, 5, 13, 14, 16, 18-20, $23-26,40,46,56,58,63,97,98$,

$100,102,120,127,134$

anxiogène, 40

contrôlable et non contrôlable, 20

de la vie quotidienne, 86, 125, 126

environnemental, 33

existentiel, 4, 5, 13, 16, 18-22, 2426

négatif/positif, 20

non normatif/normatif, 22

stressant, 19, 21, 46, 76, 77, 98-100

évitement, 4, 16, 23, 26, 56, 59-62,

$65,66,68,73,79,81,82,87,99$,

$107,127,139,146-148$

cognitif, 66, 138

passif, 16

exercice physique, 66

exosystème, 104, 116

expérience sociale, 32, 40, 44

expérience traumatique, 71 
expression émotionnelle, 42, 135, 137 externalisation, 77, 79, 84, 148

\section{$\boldsymbol{F}$}

facteur

compensatoire, 85

culturel, 105

d'adversité, 5, 85

de protection, $5,7,16,21,25,27$, $68,72,73,85,86,89$

de risque, $34,71,72,76,85,88,89$

de stress, 3, 4, 6, 20, 96, 101, 106, 116,124

de vulnérabilité, 27, 68

familial, 131

modérateur, 73

motivationnel, 123

personnel, 40, 97

proximal/distal, 89

psychologique et situationnel, 22, 40

familial, 3, 5, 7, 17, 27, 44, 45, 49,

$55,58,68,85,87,91,97,100$,

$102,105,108,111,113,115,121$,

124-126

famille, 3, 7, 17, 18, 27, 49, 55, 65, $68,72,75-78,80,84-86,88,89$, 93-113, 115, 116, 118-124, 126, $127,138,150,154$

à risques, $75-77$

aberrante, $78,84,85$

compétente, $78,84,85$

monoparentale, 118

résiliente, $78,84,85$

sans risques, 77

vulnérable, 78, 84

Family Need Survey, 107

fiabilité, 8, 131-133

fibrose kystique, 24, 138

flexibilité, 62

fonctionnement

comportemental, 36, 42, 49

familial, 96, 102, 104-106, 112, 122

social, 76,84

\section{$\boldsymbol{G}$}

garderie, $18,42,43,45,69,74-77$

gestion des émotions, 60 gestion du stress, 13, 16

glande

glucocorticoïde, $6,16,22,34,37,44$

médullosurrénale, 6, 14, 33

pituitaire, 34

surrénale, 32, 37

glucose, 35

groupe de pairs, 3, 6, 8, 18, 31, 40, 41,49

groupe à risques, 71, 85

\section{$\boldsymbol{H}$}

hémoglobine, 143

hippocampe, 37

homéostasie, 3

hôpital, 138, 140, 141

hormone, 4, 14, 15, 33, 34, 51

endocrinienne, 47

hospitalisation, 65, 77, 137-142, 147, 151

hostilité, 47

HPS, 34, 35, 38-40, 42-45, 47, 49, 51

humeur, 144

dépressive, 23, 137

hypocondrie, 57

hypophyse, 15,34

hypothalamo-hypophyso-surrénalien, 46

hypothalamus, 14,34

\section{I}

idéation suicidaire, 23, 137

IDR, 113, 115, 120

image positive de soi, 56

impact social, 41

inadaptation, $71,73,98$

incontrôlable, 61, 67, 79

indice de difficultés-ressources (IDR), 113

indice neuroendocrinologique, 44

inhibition

comportementale, 40, 42

parentale, 94

inquiétude, 147

instrument, 4, 6-8, 15, 19, 23, 25, 26, $39,42,45,48,50,64,65,67,76$,

79, 81, 96, 98, 101, 105, 106, 108, 
$111,113,115,116,118,125,131$ $134,142,145-149$

de dépistage, 8

de mesure, 111, 125, 132

standardisé, 96, 106, 125, 126

intégration sociale, 7,41

internalisation, 44, 77-79, 84, 88, 148

interprétation de la situation, 95

intervenant, 88, 112, 116

intervention, $55,62,65,67,85,88$, $89,97,99,101,103,110,111,118$, $120,123,146,147,149$

éducative, 120, 150

familiale, 111

psychologique, 103, 149

psychosociale, 88

préventive, 71, 85

invincible/invulnérable, 7, 71

irritabilité, 25

isolement social, 42, 137, 138

\section{$\boldsymbol{J}$}

JHLES, 20

jugement personnel, 95

\section{K}

Kidcope, 8, 67, 131-139, 142, 145-149, 151

\section{$\boldsymbol{L}$}

Life Event Checklist, 20

locus of control, 27

lutte ou fuite, 16

\section{M}

macro- ou micro-stresseur, 27

macrosystème, 104

maladie, 3, 5, 8, 11, 15, 16, 22, 23,

$38,129,131,132,138-146,149-151$

chronique, 24, 129, 131, 132, 138-

$140,142-146,149-151$

physique, 3

sévère ou chronique, 139, 140,

$142,145,149$

manière de faire face, $3,12,32,55,131$ marqueur biologique, 46

mécanisme

adaptatif, 5, 55, 63

de défense, 12, 23, 57, 58, 94, 95

physiologique, 6, 33

régulatoire, 31

mémoire, 35

menace, 3, 6, 11, 13, 14, 47, 56-58, 64, 65,

mésosystème, 104

mesure standardisée, 131

mesure biologique/physiologique, 6 , $15,36,50$

métastratégie, 123

microsystème, 104, 111, 116

milieu familial, 8, 85, 96, 102, 106, 115

milieu physique et éducatif des enfants, 115,116

minéralo-corticoïde, 22

modèle

clinique, 96, 112

de l'inhibition de l'action, 16

de médiation, 17

de modération, 17

de régression, 74

écologique, 7, 104

immunologique, 16, 23

organisationnel, 102, 106

psychodynamique, 58

psychopathologique, 95

théorique de risque et de protection, 89

transactionnel, 5, 13,

monoparentalité, $74,76,77,87$

motivation, 62, 116, 119

\section{$\boldsymbol{N}$}

NBAS, 39, 53

négligence, 23, 111

niche écologique, 104, 105

niveau

de scolarisation, 97

d'engagement, 42

intellectuel, 107

socioéconomique, 74,76

noradrénaline, 14, 33, 34

nouveauté sociale, 41, 43-45, 48, 49 


\section{$\boldsymbol{O}$}

observation, $7,25,38,41,43,49,50$, $67,71,74,75,95,97,106,110$, $115,116,123,125,126$

optimisme, 5, 7, 59, 62, 68

organisation comportementale, 39, 53 organisation sociale, 95

\section{$\boldsymbol{P}$}

pairs, 19, 32, 41-45, 47, 48, 55, 63, $64,68,72,75,77,89,105,150$

Parenting Stress Index, 125

partenariat, 107, 111

passivité, 14, 90, 117, 126

pathologie mentale, 95

patron de stratégies, 146

pensée magique, 61, 67, 132, 135-137, 139-141, 145

perception, $13,14,16,22,61,64,98$, $100,101,105,149$

performance scolaire, 24

personnalité, 7, 13, 23, 55, 95, 119

perspective

biosociale, 50

écologique, 102

temporelle, 105

perte, 14, 15, 18, 58, 68, 71, 104

phénomène neurophysiologique et comportemental, 36

phénylcétonurie, 38

physiologie, 12, 14, 15, 25, 31-33, 36, $37,42,50$

physiothérapie, 97

physique, 3, 5-7, 11, 19, 20, 23, 25, $26,32,36,55,63,65,68,104,115$

plan d'intervention, 111

plasticité biocomportementale/

biologique, 50, 51

pleurs, 38

prédisposition

génétique, 37

individuelle, 142, 143

prévention, $25,88,89,149,150$

problème

d'internalisation ou

d'externalisation, 48, 78 d'adaptation/d'inadaptation, 48, 77

de comportement, 24, 43, 48, 71, $77,88,96,101,126$

familial, 74

lié à la maladie chronique, 144

lié à la vie quotidienne, 139, 142145

physiologique, 32

procédure sociométrique, 53

procédure médicale, 147

processus, $11,13,31,33-36,39,41$, $48-50,56,57,71,84,85,89,94$, $98,102,104,106,111,112,116$, $119,121-124,127,138,140,144$, 148,150

adaptatif, 106, 107, 131

biocomportemental/biologique, 42, 51

d'adaptation, 72, 111

de catégorisation et de référence, 117

de coping, 59, 60, 98

dynamique, 57, 58, 72

endogène et exogène, 32

hormonal, 50

physiologique et comportemental, 31,32

professionnel, 93, 96, 111, 113, 118-

$121,145,151$

profil

comportemental, 50, 77

individuel, 53

socioaffectif (PSA), 76, 78, 84, 88

programme

d'intervention, 84, 89, 110

éducatif efficace, 106

projection, 57

propriété psychométrique, 8, 132

protection, 75,88

psychanalyse, 12, 94, 95

psychobiologie, 31-34, 36

psychologie, 3, 4, 6, 8, 11, 21, 25, 33, $35,65,94,97,120$

clinique, 94

pédiatrique, 146, 147

psychologue, 19, 20, 68, 142, 151

psychosomatique, 32 
puberté, 19, 68

pulsation du cœur, 13

$Q$

questionnaire, $8,19,20,26,76-81$, $84,98,117,123$

\section{$\boldsymbol{R}$}

rationalisation, 94

réaction, 4, 6, 7, 11-14, 26, 32, 36, 51, $53,63,68,95,99,102,111,119$,

$126,127,150$

adrénocorticale, 36

au plan cognitif, 14

au stress, 44, 117

biologique, 4, 6, 12, 62

comportementale, 32

d'adaptation, 26, 107, 123

d'anxiété, 13

de coping, 126

de fuite ou de combat, 11

émotionnelle, 7, 14, 46, 102, 112,

$118-120,122$

endocrinienne, 33

familiale, 94, 107, 121

physiologique, 32, 33, 35

positive, 119

psychologique, 120

réactivité, 34, 40, 42, 49, 115

cardiaque, 34

émotionnelle, 44

endocrinienne, 40

réajustement psychosocial, 20

recherche d'informations et de

soutien, $60,65,66,79,81,82,86$,

121,141

recherche et suppression

d'informations, 117

réconfort, 66

réévaluation de la situation, 65, 117

réévaluation positive, 65,79

réflexion, 58, 117

régression, 12, 17, 25, 115, 146, 148

régression multiple, 17,146

régulation, $6,31,32,34,36-38,40-45$, $49,50,58,60,65$ biocomportementale, $31,32,37$, $40,42,44,46,48,50,51$

comportementale et hormonale, $31,34,37,43$

des émotions, 35, 40, 58, 67, 68, $132,135,137,143-145$

endocrinienne/neuroendocrinienne, $40,41,45,49$

physiologique, $6,31,36,40,42$, 44-46, 49

relation socioaffective, 72

relation familiale, 77

relation interpersonnelle, 37

relaxation, 67

répertoire comportemental, 31, 38, $44,49,53$

réponse, 3, 4, 6, 11-14, 16, 29, 33, 36$39,41,44,55,57,60,62,63,67$, $68,76,80-82,88,93-95,106,110$, $116,118,119,121,122,132,134$, $146,150,152$

adaptative, 61

biologique/physiologique, 4, 13, 50

comportementale, 44, 58

coping, 6, 55-59, 64-66

émotionnelle, 6, 33, 58, 67, 119

endocrinienne, 37

physiologique non spécifique, 6 , $11-13,39$

subjective, 4

réseau de soutien social formel/

informel, 97, 105

réseau social, 16, 53, 66, 102, 116, 119

résignation, 15, 60, 132, 135, 139, 143,145

résilience, $3,5,7,8,27,63,69,71$ $76,79,82,85-87$

résistance, 4, 7, 34, 68, 85, 88, 89

résolution, 58-60, 62, 65-68, 79, 81,

$82,87,97,117,136$

de problème, 58-60, 66-68, 79, 81, $82,132,133,135,136,138$, $139,141,145,148,150$

respiration, 13

ressource, 4, 7, 12-14, 17, 21, 27, 51, $56,57,59,62,66,68,72,73,75$, 
95-101, 104, 106, 107, 112, 113, $115,116,119,121,122,143,149$ adaptative, 51

biologique/matérielle/ environnementale, 62

de coping, 57, 59, 62

familiale, 99, 115

financière, 97

interne et externe, 17, 72, 73, 75, 98

personnelle et environnementale, $7,17,56-58,62,63$

psychologique, 116

restructuration cognitive, $65,67,68$, $132,137,138,143,144$

retrait, $41,42,117,125,137$

social, 132, 136-138, 140, 141

risque, $68,71,73-75,77,78,84,85,89$

rôle, 34, 104, 111, 122, 124

médiateur, 22

rumination, 14

rythme cardiaque, $15,34,39,40,45$, 62

rythme circadien/ultradien, 35, 41, 45,49

\section{$\boldsymbol{S}$}

salive, $34,35,41,49,53$

sang, $15,33-35,38$

santé, 5, 6, 11, 16, 19, 21-23, 26, 27, $34,35,60,63,68,105,131,132$, $136,138,140,144,145,149,151$ mentale et physique, 6, 11, 63, 68, 73,151

score

d'adversité familiale, 76

$\mathrm{d}^{\prime}$ externalisation/d'internalisation, 77

factoriel, 134, 146

sécurité, 115,127

Self-Reported Coping Survey, 148 sentiment

d'appartenance, 143

d'autoefficacité, 62

de compétence, 111

de perte, 57

séparation mère-enfant, 37 service, 75, 93-95, 97, 103, 104, 108, $109,111,116,149$

d'aide, 93, 120

destiné aux familles, 94, 95

spécialisé, 97, 110

signification accordée aux situations stressantes, 56, 97

situation, 4, 6, 7, 12, 14, 17, 23, 33, $38-40,42-44,46,49-51,56-58,60-$ 62, 64-68, 79, 86, 87, 94, 95, 97, 100-104, 106, 110, 113, 117-119, 121-123, 125, 127, 131, 132, 134, $136,140,142-144,147,148,150$, 152

contrôlable/incontrôlable, 65, 67 de nouveauté, 43, 44, 49

stressante, 3, 4, 12, 14, 18, 55, 59$62,64,65,79,86,88,93,131$, 135,149

SNA, 11

SNC, 16

social, 11, 16, 27, 37, 41, 55, 56, 61, $62,64,89,90,97,101,104,107$, $108,115,116,125,141,151$

socialisation, 73

souci, 14, 20, 21, 101

source d'informations, 66

source de stress, 5, 6, 17, 18, 57

soutien, 97, 105, 108, 109

aux familles, 112

formel et informel, 7, 108, 121

formel non professionnel/

professionnel, 108, 109

social, $5,7,60,65,66,72,79,86$,

90, 132, 135-138, 141, 143, 144, 148

SPT, 23

stéroïde, 36

stimuli aversifs, 11

stimulus, 3, 4, 11-14, 26, 32-34, 36, 44,46 environnemental, 4 négatif, 38

stratégie, 3-8, 12, 32, 38, 39, 42, 45, $53,55,57-67,69,73,74,78-84$, $86-88,90,91,97,105,112,116-$ $127,129,131-136,139,143-151$ 
active/passive, 132

centrée

sur le problème, 61, 66, 132

sur les émotions, 61, 66, 73, 117,132

cognitive, 4, 6, 8, 27, 33, 56, 60, 64-68, 112, 117, 122, 123, 125, 139,141

cognitive/comportementale/ instrumentale, 7, 39, 59, 67, 79, $112,117,123,132,149,151$

d'adaptation, 3-7, 12, 32, 42, 53, $55,58,59,64,65,67,73,74$, 78-84, 86-88, 91, 116-118, 121, 123-126, 129, 131-151

d'adaptation individuelle, 133, 146 d'adaptation négative, 141 d'approche, 5-7, 11, 32, 41, 45, 48, $50,51,53,56-61,74,75,82,84$, $85,94,95,98-100,102,104$, $111-113,117,120,132,142$, 146-148, 150

d'approche/d'évitement, 61, 67, 82, $83,87,88,132,137$

d'auto-contrôle, 38

de distraction cognitive, 65

de résolution de problème, 86,97 , 137

de restructuration cognitive, 66 évitante, 141

instrumentale, 121, 126

préventive, 149

prosociale, 45

sociale, 66

stress, 3-9, 11-27, 29, 31-39, 41, 43-

$45,47,49,50,55,56,59-61,63$,

$67,68,73,74,76-78,84-87,91$,

93-102, 106, 107, 110, 112, 113,

$115,116,121,122,124,125,127$,

143,144

au travail, 18

biologique, 3, 6, 8, 36

chronique, 15, 36, 76, 124

d'urgence, 15

émotionnel, 22

endogène ou exogène, 4

familial, 3, 5, 7, 8, 27, 76, 77, 79, 99, 101 financier, 57

lié à la santé, 77

majeur, 77

post-traumatique, 23,26

psychologique et émotionnel, 4-6,

$9,13,32$

scolaire, $19,57,67$

social, 6

stress-détresse, 6, 73

stresseur, 7, 11-14, 16, 17, 19-22, 25-

$27,32,56,59-62,66,98,126,131-$

$135,139-151$

interpersonnel, 67

majeur/mineur, 19, 21-23

scolaire, 67

structure

d'aide aux familles, 95

éducative, 123

factorielle, 79, 146, 147

style d'adaptation, 51, 55, 58, 59, 146

substitution, 94

succès scolaire, 24

support social, 17, 25, 27

support formel/informel, 109

surprotection, 93

symptôme

dépressif, 63

physiologique, 32

psychologique, 63

syndrome général d'adaptation (SGA), 12

système

adrénocortical, 37-40, 44

biologique, 45

biosocial, 51

cardiovasculaire, 34

corticosurrénal, 40

émotif, 38

écologique, 118

endocrinien, 35, 36, 38, 40-42, 44, 49, 50

juridique, 104

hypophysaire, 14,15

immunitaire, 35

limbique, 35

nerveux autonome sympathique et parasympathique, 34

nerveux central, 44 
nerveux sympathique, 14, 12, 33 neuroendocrinien et comportemental, 6, 33, 47 physiologique et comportemental, 37

\section{$\boldsymbol{T}$}

tâche, 33, 39, 65, 73-76, 139 développementale, 57, 64, 72 éducative, 111, 113

taux de glucose, 34

taxonomie, $41,81,88$

technique d'échantillonnage, 35, 75

technique du tamisage, 75

tempérament, 7, 27, 40, 43, 44, 53, 59,72

tension, 5, 21, 57, 58, 90, 94

chronique, $5,21,24$

théorie transactionnelle du stress, 56 tourment, 21

tracas quotidiens, 4, 5, 18-22, 25, 26

trait, 13, 58, 59, 81, 134, 143-145

traitement, 38, 53, 65, 108, 131, 138, $140,144,150$

transaction, 33

travail, 8, 11, 18, 55, 62, 64, 77, 88, $105,110,112,116,142,151$

travail clinique, 129

trisomie, 21, 94, 103, 110, 124

tristesse, 105, 119, 136-138, 141

trouble

alimentaire, 24

d'adaptation, 96

d'anxiété, 47

de comportement, 24, 26, 46, 87, $100,105,107,109,111$ de conduite, 25, 47

d'hyperactivité, 47

de la personnalité, 47, 94

du déficit d'attention, 25

du sommeil, 14, 23, 25

émotionnel, 24

psychologique et physique, 55

\section{$\boldsymbol{U}$}

ulcère d'estomac, 14, 15, 32

urine, 33-35

$\boldsymbol{V}$

vaccination, 38

validité, $8,26,39,123,131,133,134$, 148

variable

démographique, 108

intermédiaire, 17

médiatrice, 17

modératrice, 5, 17, 27, 143

situationnelle, 144

variation

endocrinienne, 43

hormonale, 36

intra-individuelle, 51, 57

violence, 46

voie nerveuse, 33

vulnérabilité, $4,5,26,79,84,85$

vulnérabilité génétique, 71

$\boldsymbol{W}$

Ways of Coping Questionnaire, 79, 80 